\title{
A Structural Result for Personalized PageRank and its Algorithmic Consequences
}

\author{
DANIEL VIAL, University of Michigan, USA \\ VIJAY SUBRAMANIAN, University of Michigan, USA
}

Many systems, such as the Internet, social networks, and the power grid, can be represented as graphs. When analyzing graphs, it is often useful to compute scores describing the relative importance or distance between nodes. One example is Personalized PageRank (PPR), which assigns to each node $v$ a vector whose $i$-th entry describes the importance of the $i$-th node from the perspective of $v$. PPR has proven useful in many applications, such as recommending who users should follow on social networks (if this $i$-th entry is large, $v$ may be interested in following the $i$-th user). Unfortunately, computing $n$ PPR vectors exactly for a graph of $n$ nodes has complexity $O\left(n^{3}\right)$, which is infeasible for many graphs of interest.

In this work, we devise a scheme to estimate all $n$ PPR vectors with bounded $l_{1}$ error and complexity $O\left(n^{\bar{c}}\right)$, where $\bar{c}<2$ depends on the degrees of the graph at hand, the desired error tolerance, and a parameter that defines PPR. This improves upon existing methods, the best of which have complexity $O\left(n^{2} \log n\right)$ in our setting. Our complexity guarantee holds with high probability, for certain choices of the PPR parameter, and for a certain class of random graphs (roughly speaking, the sparse directed configuration model with heavy-tailed in-degrees); our accuracy guarantee holds with probability 1 and for arbitrary graphs and PPR parameters. The complexity result arises as a consequence of our main (structural) result, which shows that the dimensionality of the set of PPR vectors scales sublinearly in $n$ with high probability, for the same class of random graphs and for a notion of dimensionality similar to matrix rank. It is this coupling of the PPR vectors for the nodes on a common underlying graph that allows for estimating them faster. Hence, at a high level, our scheme is analogous to (but distinct from) low-rank matrix approximation. We also note that our scheme is similar to one that was proposed in [Jeh and Widom 2003] but lacked accuracy and complexity guarantees, so another contribution of our paper is to address this gap in the literature.

ACM Reference Format:

Daniel Vial and Vijay Subramanian. 2019. A Structural Result for Personalized PageRank and its Algorithmic Consequences. Proc. ACM Meas. Anal. Comput. Syst. 3, 2, Article 25 (June 2019), 88 pages. https://doi.org/10. $1145 / 3326140$

\section{INTRODUCTION}

Many natural and man-made systems can be represented as graphs, sets of objects (called nodes) and pairwise relations between these objects (called edges). These include the brain, which contains neurons (nodes) that exchange signals through chemical pathways (edges), the Internet, which contains websites (nodes) that are connected via hyperlinks (edges), etc. To study graphs, researchers in diverse domains have used Personalized PageRank (PPR) [28]. Informally, PPR assigns to each node $v$ a vector $\pi_{v}$, where $\pi_{v}(w)$ describes the importance or relevance of node $w$ from the perspective of $v$. PPR has proven useful in many applications, both practical and graph-theoretic.

Authors' addresses: Daniel Vial, University of Michigan, Ann Arbor, MI, USA, dvial@umich.edu; Vijay Subramanian, University of Michigan, Ann Arbor, MI, USA, vgsubram@umich.edu.

Permission to make digital or hard copies of all or part of this work for personal or classroom use is granted without fee provided that copies are not made or distributed for profit or commercial advantage and that copies bear this notice and the full citation on the first page. Copyrights for components of this work owned by others than ACM must be honored Abstracting with credit is permitted. To copy otherwise, or republish, to post on servers or to redistribute to lists, requires prior specific permission and/or a fee. Request permissions from permissions@acm.org.

(C) 2019 Association for Computing Machinery.

2476-1249/2019/6-ART25 \$15.00

https://doi.org/10.1145/3326140

Proc. ACM Meas. Anal. Comput. Syst., Vol. 3, No. 2, Article 25. Publication date: June 2019. 
Examples include recommending who a user should follow on Twitter [29] ( $v$ may wish to follow $w$ if $\pi_{v}(w)$ is large) and local graph partitioning [4] (the set of nodes $w$ with large $\pi_{v}(w)$ can be viewed as a community surrounding $v$ ). Unfortunately, for a graph of $n$ nodes, computing all $n$ PPR vectors has complexity $O\left(n^{3}\right)$, which is infeasible for massive graphs arising in modern domains.

In this work, we provide an algorithm to estimate all PPR vectors with bounded $l_{1}$ error for general graphs and with complexity $O\left(n^{\bar{c}}\right)$ (for some $\bar{c}<2$ ) for a class of random graphs. These algorithmic results arise as a consequence of a structural result, which states the effective dimension of the set of PPR vectors scales sublinearly in $n$. More specifically, our contributions are as follows:

(1) In Section 4, we prove the dimensionality of the set of PPR vectors scales sublinearly in $n$ with high probability, for a certain class of random graphs and for a notion of dimensionality similar to rank (Theorem 1). Put differently, we argue that the effective dimension of this set is much less than $n$; this occurs despite the fact that the matrix containing these vectors has rank $n$. This class of graphs can be roughly described as the directed configuration model with sparse but heavy-tailed in-degrees. The notion of dimensionality we study is the smallest number of "hub" nodes such that the PPR vectors of all other nodes are close to linear combinations of the hub PPR vectors.

(2) In Section 5, we show this notion of dimensionality relates closely to the complexity of estimating the set of PPR vectors. More specifically, we use our structural result to show that this set can be accurately estimated in the $l_{1}$ norm with complexity $O\left(n^{\bar{c}}\right)$ for some $\bar{c}<2$, with high probability and for the same class of graphs (Theorem 2). Broadly speaking, this scheme leverages the low dimension of the PPR vectors in a manner analogous to low-rank matrix approximation. To the best of our knowledge, our scheme improves upon all existing bounds for this task, the most competitive of which are $O\left(n^{2} \log n\right)$ in our setting. We also note that PPR vectors are nonnegative and sum to 1 ; hence, they are distributions, so bounding $l_{1}$ error (equivalent to bounding error in the total variation norm) is a natural objective.

(3) The estimation scheme we analyze is similar to one that was proposed (but not analyzed) by Jeh and Widom in [33] and that has been widely used in practice. Hence, another contribution is to offer theoretical evidence for the empirical success of the algorithm from [33].

(4) While Theorems 1 and 2 apply to a class of random graphs, we show empirically in Section 6 that our dimensionality measure is small relative to $n$ for real graphs. Hence, we argue that the dimension of the set of PPR vectors is small in general. This also suggests that the complexity of estimating these vectors becomes much smaller when one accounts for dependencies between the vectors that arise from the common underlying graph.

(5) Additionally, we believe the class of random graphs considered contains realistic models of real-world networks. As an example, in Section 7.3 we provide a model for a graph like Twitter, which contains a few nodes with huge in-degrees - modeling celebrities with millions of Twitter followers - and many nodes with moderate in-degrees - modeling "normal" users with dozens or hundreds of followers. We also discuss various other aspects of our analysis throughout Section 7, including a geometric interpretation of our structural result and a connection to Markov chain mixing times.

The paper is organized as follows. We begin in Section 2 with preliminary definitions. In Section 3, we discuss related work. Sections 4-7 follow the outline above. We close in Section 8.

\section{PRELIMINARIES}

We begin by defining the main ingredients of the paper. Most notation is standard or defined as needed, but we note the following is often used: for $x \in \mathbb{R}^{n}$ and $J \subset\{1,2, \ldots, n\}$, we set 
$x(J)=\sum_{j \in J} x(j)$, we let $e_{J} \in\{0,1\}^{n}$ satisfy $e_{J}(j)=1(j \in J)$ (where $1(\cdot)$ is the indicator function), and we write $e_{j}=e_{\{j\}}$ for simplicity.

\subsection{Directed configuration model (DCM)}

We consider a random graph model called the directed configuration model (DCM). For the DCM, we are given realizations of random sequences $\mathbf{N}_{n}=\left\{N_{v}\right\}_{v \in V_{n}}$ and $\mathbf{D}_{n}=\left\{D_{v}\right\}_{v \in V_{n}}$ satisfying $N_{v}, D_{v} \in \mathbb{N} \forall v \in V_{n}$ and $\sum_{v \in V_{n}} N_{v}=\sum_{v \in V_{n}} D_{v} \triangleq L_{n}$ (here $\left.V_{n}=\{1,2, \ldots, n\}\right) .{ }^{1}$ We will refer to $\left(\mathbf{N}_{n}, \mathbf{D}_{n}\right)$ as the given degree sequence. Our goal is to construct a directed graph $G_{n}=\left(V_{n}, E_{n}\right)$, such that $v \in V_{n}$ has in- and out-degree $N_{v}$ and $D_{v}$, respectively. For this, we first assign $N_{v}$ incoming half-edges and $D_{v}$ outgoing half-edges to each $v \in V_{n}$; we call these half-edges instubs and outstubs, respectively. We then randomly pair half-edges to form edges in a breadth-first search fashion that proceeds as follows:

- Let $s \sim V_{n}$ uniformly. For each of the $D_{s}$ outstubs assigned to $s$, sample an instub uniformly from the set of all instubs (resampling if the sampled instub has already been paired), and pair the outstub and instub to form an edge out of $s$.

- Let $A_{1}=\left\{v \in V_{n} \backslash\{s\}:\right.$ an outstub of $s$ was paired with an instub of $\left.v\right\}$. For each $v \in A_{1}$, pair the $D_{v}$ outstubs assigned to $v$ in the same manner $s$ 's outstubs were paired.

- Continue iteratively until all half-edges have been paired. Namely, during the $(m+1)$-th iteration we pair the outstubs of all $v \in A_{m}$, where $A_{m}$ is the set of nodes at distance $m$ from $s$ (those $v \in V_{n}$ for which the shortest path from $s$ to $v$ has length $m$ ).

We define this procedure more formally in Appendix A.2. For now, the important points to remember are that the initial node $s$ is chosen uniformly at random from $V_{n}$, and that, at the end of the $m$-th iteration, the $m$-step neighborhood out of $s$ has been constructed. We emphasize the resulting graph will be a multi-graph in general, i.e. it will contain self-loops (edges $v \rightarrow v$ for $v \in V_{n}$ ) and multi-edges (more than one edge from $v \in V_{n}$ to $w \in V_{n}$ ). ${ }^{2}$

\subsection{Personalized PageRank (PPR)}

To define PPR, we require some notation. First, let $M$ denote the adjacency matrix for some realization of the DCM, i.e. $M(i, j) \in\left\{0,1, \ldots, D_{i}\right\}$ is the number of directed edges from $i$ to $j$, for each $i, j \in V_{n}$. Next, let $P$ be the row stochastic matrix with $P(i, j)=M(i, j) / D_{i} \forall i, j \in V_{n}$. Finally, let $\alpha_{n} \in(0,1)$, and let $1_{n}$ denote the length- $n$ vector of ones. We then have the following.

Definition 1. For $v \in V_{n}$, let $P_{v}=\left(1-\alpha_{n}\right) P+\alpha_{n} 1_{n} e_{v}^{\top}$. Then the PPR row vector corresponding to $v$, denoted $\pi_{v}$, is the stationary distribution of the Markov chain with transition matrix $P_{v}$.

As stated in Definition 1, we treat $\pi_{v}$ as a row vector; all other vectors are treated as column vectors, unless explicitly stated otherwise. We also note that $M, P, P_{v}$, and $\pi_{v}$ appearing in the previous paragraph all depend on $n$. However, to avoid cumbersome notation, we do not explicitly denote this, and the dependence on $n$ will be clear from context.

The Markov chain described in Definition 1 has the following dynamics: at each step, follow a uniform random walk with probability $\left(1-\alpha_{n}\right)$, and jump to $v$ with probability $\alpha_{n}$. This motivates an interpretation of PPR as a centrality measure of the nodes $V_{n}$ from the perspective of $v$. To see this, let $\left\{X_{i}\right\}_{i=0}^{\infty}$ denote the Markov chain with transition matrix $P$, let $L \sim \operatorname{Geometric}\left(\alpha_{n}\right)$, and let $\mathbb{E}_{G_{n}}[\cdot]$ denote expectation with some realization of $G_{n}$ held fixed. Then, as proven in Appendix

\footnotetext{
${ }^{1}$ For example, in Section 7.3, we let $\mathrm{N}_{n} \sim f_{\text {in }}$ i.i.d. for a given distribution $f_{\text {in }}$; we then realize $\mathbf{D}_{n}$ conditional on $\sum_{v \in V_{n}} N_{v}$ in a manner that guarantees $\sum_{v \in V_{n}} D_{v}=\sum_{v \in V_{n}} N_{v}$. Alternatively, [22] provides an algorithm to let $\mathrm{N}_{n} \sim f_{\text {in }}$ i.i.d., $\mathbf{D}_{n} \sim f_{\text {out }}$ i.i.d. for given distributions $f_{\text {in }}, f_{\text {out }}$, then to modify $\mathbf{N}_{n}, \mathbf{D}_{n}$ to guarantee $\sum_{v \in V_{n}} D_{v}=\sum_{v \in V_{n}} N_{v}$.

${ }^{2}$ In [22], the authors provide conditions under which a simple graph results with positive probability as $n \rightarrow \infty$, but these are stronger than the conditions we require to prove our main result, so we will instead assume $G_{n}$ is a multi-graph.
} 
D.1.1, (1) holds, which implies $\pi_{v}(u)$ is large when $u$ is frequently visited (a notion of centrality) on Geometric $\left(\alpha_{n}\right)$-length walks starting at $v$ (a notion of $v$ 's perspective).

$$
\pi_{v}(u)=\alpha_{n} \mathbb{E}_{G_{n}}\left[\sum_{i=0}^{L-1} 1\left(X_{i}=u\right) \mid X_{0}=v\right] \forall u \in V_{n} .
$$

We note the typical definition of PPR assumes $\alpha_{n}$ is a constant independent of $n$; in contrast, we take $\alpha_{n}=\Theta(1 / \log n)$. We argue in Section 4.2 that this is appropriate when considering the asymptotic behavior of PPR on the DCM. Specifically, we argue that the size of the set of nodes that are important to $v$ grows with the graph, but grows slowly enough that a notion of $v$ 's perspective remains, when $\alpha_{n}=\Theta(1 / \log n)$. (In contrast, this set has constant size when $\alpha_{n}$ is constant.) Additionally, we note the spectral gap of $P_{v}$ is lower bounded by $\alpha_{n}$, so $\alpha_{n} \rightarrow 0$ as $n \rightarrow \infty$ results in this lower bound vanishing asymptotically. We remark that a line of work by Boldi et al. $[11,12]$ analyzed the limit of PPR as $\alpha_{n} \rightarrow 0$ for a fixed graph $G_{n}$; in contrast, we fix a value of $\alpha_{n}$ for each $G_{n}$. Finally, we will further motivate our choice of $\alpha_{n}$ in Section 7.4 via recent results on the mixing time of random walks on random graphs $[16,17]$.

Before proceeding, we emphasize the distinction between PPR and the more commonly known notion of PageRank, which we refer to as global PageRank for clarity. In short, global PageRank is the average of all PPR vectors, i.e. $\sum_{v \in V_{n}} \pi_{v} / n$. Hence, global PageRank is a centrality measure from the perspective of a uniformly chosen node. More generally, given a distribution $\sigma_{n}$ on $V_{n}$, the PPR corresponding to $\sigma_{n}$ is $\pi_{\sigma_{n}}=\sum_{v \in V_{n}} \sigma_{n}(v) \pi_{v}$.

\subsection{Dimensionality measure}

Our main focus is the dimensionality of the set of PPR vectors, $\left\{\pi_{v}\right\}_{v \in V_{n}}$. A standard measure of this dimension is $\operatorname{rank}\left(\Pi_{n}\right)$, where $\Pi_{n}$ is the matrix with rows $\left\{\pi_{v}\right\}_{v \in V_{n}} ;$ however, $\operatorname{rank}\left(\Pi_{n}\right)=n \forall n \in \mathbb{N}$, so we will instead consider a different notion of dimensionality. ${ }^{3}$ This notion is motivated by the following observation: the rank of the matrix with rows $\left\{x_{i}\right\}_{i \in I}$ (where $I$ is some finite set) can be bounded by $\left|X^{\prime} \cup X^{\prime \prime}\right|$, where $X^{\prime} \subset\left\{x_{i}\right\}_{i \in I}$ and $X^{\prime \prime}=\left\{x_{i} \notin X^{\prime}: x_{i}\right.$ is not a linear combination of $\left.X^{\prime}\right\}$. We will relax this slightly, by only including in $X^{\prime \prime}$ those $x_{i} \notin X^{\prime}$ that are not "close" to a linear combination of $X^{\prime}$. Mathematically, for $\epsilon>0$ we define

$$
\min _{K_{n} \subset V_{n}} \Delta\left(K_{n}, \epsilon\right)=\left|K_{n}\right|+\mid\left\{v \in V_{n} \backslash K_{n}: B_{v}\left(K_{n}, \epsilon\right) \text { holds }\right\} \mid,
$$

where $B_{v}\left(K_{n}, \epsilon\right)$ is the event

$$
\left\{\inf _{\left\{\beta_{v}(k)\right\}_{k \in K_{n}} \subset \mathbb{R}}\left\|\pi_{v}-\left(\sum_{k \in K_{n}} \beta_{v}(k) \pi_{k}+\alpha_{n} e_{v}^{\top}\right)\right\|_{1} \geq \epsilon\right\} .
$$

We offer several remarks on this definition. First, as will be discussed in Section 5, (2) suggests an algorithm for estimating all PPR vectors: we first estimate $\pi_{v}$ for each $v \in K_{n}$ and $v \notin K_{n}$ such that $B_{v}\left(K_{n}, \epsilon\right)$ holds; we then approximate $\pi_{v}$ for other $v$ as a linear combination of the $\left\{\pi_{k}\right\}_{k \in K_{n}}$ estimates. Under this scheme, (2) is the number of PPR vectors estimated directly (i.e., not as linear combinations); we will argue this direct estimation dominates the scheme's complexity, and thus the scheme's complexity scales with (2). Second, we note (2) differs slightly from the quantity $\left|X^{\prime} \cup X^{\prime \prime}\right|$ defined in the previous paragraph, since in (3) the estimate of $\pi_{v}$ is a linear combination

\footnotetext{
${ }^{3}$ To see why $\operatorname{rank}\left(\Pi_{n}\right)=n$, first suppose $\left(I-\left(1-\alpha_{n}\right) P\right)$ is not invertible. Then $\left(I-\left(1-\alpha_{n}\right) P\right) \xi=0$ for some $\xi \neq 0$, so $\xi /\left(1-\alpha_{n}\right)=P \xi$. But, by the Perron-Frobenius theorem, $P$ cannot have eigenvalue $1 /\left(1-\alpha_{n}\right)>1$, since it is row stochastic. Hence, $\left(I-\left(1-\alpha_{n}\right) P\right)$ is invertible, so by $(4), \Pi_{n}$ is also invertible.
} 
plus the term $\alpha_{n} e_{v}^{\mathrm{T}}$. This latter term is included because it is a known component of $\pi_{v}$, independent of the graph structure encoded by $P$. Indeed, using Definition 1 , we have

$$
\pi_{v}=\left(1-\alpha_{n}\right) \pi_{v} P+\alpha_{n} e_{v}^{\top} \Rightarrow \pi_{v}=\alpha_{n} e_{v}^{\top}\left(I-\left(1-\alpha_{n}\right) P\right)^{-1}=\alpha_{n} e_{v}^{\top}+\alpha_{n} e_{v}^{\top} \sum_{i=1}^{\infty}\left(1-\alpha_{n}\right)^{i} P^{i}
$$

Third, we note $l_{1}$ distance is a reasonable choice in (3) because PPR vectors are distributions over $V_{n}$, $l_{1}$ distance is twice total variation distance, and total variation is a standard distance for comparing distributions. Finally, we note (2) bears some resemblance to low-rank matrix approximation and nonnegative matrix factorization (NMF); we discuss this in Section 5.2.

Our structural result (Theorem 1) provides conditions on the given degree sequence $\left(\mathrm{N}_{n}, \mathrm{D}_{n}\right)$ and the choice of $K_{n}$ under which $\Delta\left(K_{n}, \epsilon\right)$ scales sublinearly in $n$; hence, under these conditions, $\min _{K_{n} \subset V_{n}} \Delta\left(K_{n}, \epsilon\right)$ is also sublinear. (Clearly, $\left|K_{n}\right|$ being sublinear is one such condition; proving that $\mid\left\{v \in V_{n} \backslash K_{n}: B_{v}\left(K_{n}, \epsilon\right)\right.$ holds $\} \mid$ is also sublinear is highly nontrivial.) We note that we currently lack a matching lower bound for (2), so it is unclear if our bound is order optimal (or if (2) may instead scale with, for example, $\log n)$.

Moving forward, we will set $K_{n}=\left\{v \in V_{n}: U_{v}=0\right\}$, where $\mathbf{U}_{n}=\left\{U_{v}\right\}_{v \in V_{n}}$ is a random length- $n$ binary sequence that may be correlated with the given degree sequence $\left(\mathrm{N}_{n}, \mathrm{D}_{n}\right)$. Additionally, we will assume the entire tuple $\left(\mathrm{N}_{n}, \mathrm{D}_{n}, \mathrm{U}_{n}\right)$ is realized before the graph is constructed. In light of this, we emphasize that $\Delta\left(K_{n}, \epsilon\right)$ is a random variable that depends on two sources of randomness: the random sequence $\left(\mathrm{N}_{n}, \mathrm{D}_{n}, \mathrm{U}_{n}\right)$, and the random graph construction. Towards proving our main result, intermediate results will be established with $\left(\mathrm{N}_{n}, \mathrm{D}_{n}, \mathrm{U}_{n}\right)$ held fixed, after which expectation with respect to $\left(\mathrm{N}_{n}, \mathrm{D}_{n}, \mathrm{U}_{n}\right)$ will be taken. This motivates the following definitions: we let $\mathbb{E}_{n}[\cdot]=\mathbb{E}\left[\cdot \mid \mathbf{N}_{n}, \mathbf{D}_{n}, \mathbf{U}_{n}\right]$ and $\mathbb{P}_{n}[\cdot]=\mathbb{P}\left[\cdot \mid \mathbf{N}_{n}, \mathbf{D}_{n}, \mathbf{U}_{n}\right]$ denote expectation and probability with the only source of randomness being the graph construction.

\section{RELATED WORK}

Before proceeding, we comment on relationships to prior work. We focus on [33] and [21], the papers most closely related to our own. We will also return to discuss more related literature - in particular, other PPR estimation schemes and how they compare to ours - in Section 5.2.

In [33], Jeh and Widom propose a scheme for estimating the set of all PPR vectors. The scheme relies crucially on the Hubs Theorem in [33], which states that the PPR vector $\pi_{v}, v \in V_{n} \backslash K_{n}$, can be written as a linear combination of $\left\{\pi_{k}\right\}_{k \in K_{n}}$ and another vector. The Hubs Theorem is central to our results as well; an alternative formulation appears as Lemma 2 here. We also discuss the algorithm of Jeh and Widom in more detail in Section 5.2.

Unfortunately, the authors of [33] present no analysis of their scheme. Hence, it is unclear how $K_{n}$ should be chosen and how large it must be to guarantee accurate estimation. Our work addresses this shortcoming. Specifically, as discussed briefly in the introduction and in more detail in Section 5 , our dimensionality measure (2) relates to the complexity of a similar estimation scheme.

In [21], Chen, Litvak, and Olvera-Cravioto consider the limiting value of $\pi_{\sigma_{n}}$ as $\sigma_{n}$ weakly converges to a probability distribution $\sigma$. Specifically, they show that the PPR value of a uniformly chosen node is given by the solution of a recursive distributional equation (RDE) [1]. They also show (roughly) that PPR values follow a power law when in-degrees follow a power law, establishing the "power law hypothesis" that had long been observed empirically. Similar results were proven for other graph families in [36]. On the other hand, [21] was preceded by [20], where the power law hypothesis was established for global PageRank; further back, the hypothesis was studied under more restrictive assumptions in [38, 48, 49]. 
While [20, 21, 36, 38, 48, 49] share a goal of understanding the power law behavior of PPR on random graphs, our goal is to instead understand structural properties of the PPR vectors collectively, with the focus of this paper being dimensionality. Since dimensionality carries with it algorithmic implications, our work is perhaps more useful from a practical perspective when compared to this body of work. However, the analytical approaches of these works will be useful to us. Specifically, we will use a modified version of Lemma 5.4 from [21]; see Appendix A.3.

In short, our work combines the strengths of [33], which is entirely algorithmic, and [21], which is entirely analytical. Specifically, we leverage certain aspects of the analysis from [21] to obtain guarantees on an algorithm similar to that proposed in [33].

More broadly, references for PageRank and PPR include [43], in which PageRank and PPR were first proposed, and [31], an early study of PPR (there called "topic-sensitive" PageRank). Beyond [33], many other works have proposed efficient computation and estimation algorithms for PPR; a small sample includes those using linear algebraic techniques $[45,46]$, those using dynamic programming $[3,4]$, and those using randomized schemes [7, 39]. As mentioned above, Section 5.2 contains a more thorough discussion of this literature. We also point the reader to [28] for a survey of the many applications of PPR. In addition to the body of work on the power law hypothesis, analysis of PPR on random graphs includes [5], where it is shown that $\pi_{\sigma_{n}}$ can be well-approximated as a convex combination of $\sigma_{n}$ and the degree distribution for certain random graphs.

The DCM was proposed and analyzed in [22] as an extension of the (undirected) configuration model, the development of which began in $[9,14,50]$. The configuration model (and variants) have been studied in detail; for example, [47] considers graph diameter in this model, while [42] studies the emergence of a giant component.

\section{STRUCTURAL RESULT}

We next turn to our main structural result. We begin by defining our assumptions and our choice of $\alpha_{n}$ in Sections 4.1 and 4.2, respectively. We then state the result in Section 4.3.

\subsection{Assumptions}

Our main result is a consequence of a key lemma (Lemma 1), the proof of which requires Assumption 1. This assumption states that certain empirical moments of $\left(\mathrm{N}_{n}, \mathbf{D}_{n}, \mathrm{U}_{n}\right)$ exist with high probability, and furthermore, converge to limits at a certain rate.

Assumption 1. We have $\mathbb{P}\left[\Omega_{n}^{C}\right]=O\left(n^{-\delta}\right)$ for some $\delta \in(0,1)$, where $\Omega_{n}=\cap_{i=1}^{6} \Omega_{n, i}$ and for some constants $\gamma, p \in(0,1)$ and $\eta_{i}, \zeta^{*}, \lambda^{*} \in(0, \infty)$, all independent of $n$,

$$
\begin{array}{ll}
\Omega_{n, 1}=\left\{\left|\frac{\sum_{h=1}^{n} N_{h}}{n}-\eta_{1}\right| \leq n^{-\gamma}\right\}, & \Omega_{n, 4}=\left\{\left|\frac{\sum_{h=1}^{n} U_{h} D_{h}}{\sum_{h=1}^{n} U_{h}}-\zeta^{*}\right| \leq n^{-\gamma}\right\}, \\
\Omega_{n, 2}=\left\{\left|\frac{\sum_{h=1}^{n} N_{h} D_{h}}{n}-\eta_{2}\right| \leq n^{-\gamma}\right\}, & \Omega_{n, 5}=\left\{\left|\frac{\sum_{h=1}^{n} U_{h} N_{h}}{\sum_{h=1}^{n} U_{h}}-\lambda^{*}\right| \leq n^{-\gamma}\right\}, \\
\Omega_{n, 3}=\left\{\left|\frac{\sum_{h=1}^{n} U_{h} N_{h}^{2}}{n}-\eta_{3}\right| \leq n^{-\gamma}\right\}, & \Omega_{n, 6}=\left\{\left|\frac{\sum_{h=1}^{n} U_{h} N_{h}}{\sum_{h=1}^{n} N_{h}}-p\right| \leq n^{-\gamma}\right\} .
\end{array}
$$

We also have $\zeta \triangleq \eta_{2} / \eta_{1}>1$ and define $\lambda=\eta_{3} / \eta_{1}$.

We note that the constants $\zeta$ and $p$ appearing in Assumption 1 also appear in our main result, and both have simple interpretations: letting $v_{n}$ satisfy $\mathbb{P}\left[v_{n}=v\right] \propto N_{v} \forall v \in V_{n}, n \in \mathbb{N}$, it is straightforward to show $\lim _{n \rightarrow \infty} \mathbb{E}\left[D_{v_{n}} \mid \Omega_{n}\right]=\zeta$ and $\lim _{n \rightarrow \infty} \mathbb{E}\left[U_{v_{n}} \mid \Omega_{n}\right]=p$, i.e. $\zeta$ and $p$ give the limiting expected out-degree and the limiting probability of belonging to $V_{n} \backslash K_{n}$, respectively, for a node sampled with probability proportional to in-degree. (The other constants in Algorithm 1 will 
not appear in our main result, but they have similar interpretations.) We also remark that $\zeta>1$ in Assumption 1 is not necessary to establish our results but, given this interpretation, is the more interesting case; furthermore, this simplifies the statements and proofs of certain results (which otherwise would have to address the cases $\zeta>1, \zeta=1$, and $\zeta<1$ separately).

Our main result (Theorem 1) requires Assumption 2, which strengthens Assumption 1 with the requirement that $\left|K_{n}\right|$ is sublinear (an obvious requirement for sublinearity of (2)).

Assumption 2. Assumption 1 holds and, for some $\kappa \in(0,1)$ independent of $n, \mathbb{E}\left[\left|K_{n}\right|\right]=O\left(n^{\kappa}\right)$.

We note that, while Assumption 2 may appear limiting, we provide an example sequence $\left(\mathbf{N}_{n}, \mathbf{D}_{n}, \mathbf{U}_{n}\right)$ in Section 7.3 that satisfies it, and that we believe is a reasonable model for certain graphs of interest (like Twitter). Additionally, we argue in Section 7.2 that several events $\Omega_{n, i}$ appearing in Assumption 1 are essentially implied by others, and are therefore not restrictive. Finally, we believe the most important condition for sublinearity of $\Delta\left(K_{n}, \epsilon\right)$ is that $K_{n}$ contains a vanishing fraction of nodes but a non-vanishing fraction of edges (i.e. $\mathbb{E}\left[\left|K_{n}\right|\right]=O\left(n^{\kappa}\right)$ and $\Omega_{n, 6}$ holds in Assumption 1). We discuss this further in Section 7.2 and provide empirical evidence that this holds when $\left\{N_{v}\right\}_{v \in V_{n}}$ follow a power law, a common model for real-world graphs.

\subsection{Choice of $\alpha_{n}$}

As mentioned in Section 2.2, we take $\alpha_{n}=\Theta(1 / \log n)$ in this work. Having defined Assumption 1 , we choose a specific value of $\alpha_{n}$. This choice is motivated by the following proposition, which states that $s$ 's PPR concentrates in a small neighborhood surrounding $s$, and bounds the size of this neighborhood, for two choices of $\alpha_{n}$.

Proposition 1. Let $\tau \in(0,1)$ and $\rho>1$ be constants, and let $s \sim V_{n} \backslash K_{n}$ uniformly. For $l \in \mathbb{N}$, let $V_{n, s}(l)$ denote the l-step neighborhood out of s. Then the following hold:

- If $\alpha_{n}=\rho \log (1 / \tau) \log (\zeta) / \log (n)=\Theta(1 / \log n)$ and $l=\left\lceil\log (1 / \tau) / \alpha_{n}\right\rceil$,

$$
\liminf _{n \rightarrow \infty} \pi_{s}\left(V_{n, s}(l)\right) \geq 1-\tau \text { a.s., } \quad \mathbb{E}\left[\mid V_{n, s}(l) \| \Omega_{n}\right]=O\left(n^{1 / \rho}\right) .
$$

- If $\alpha_{n}=\alpha$ is a constant and $l=\lceil\log (\tau) / \log (1-\alpha)\rceil$,

$$
\liminf _{n \rightarrow \infty} \pi_{s}\left(V_{n, s}(l)\right) \geq 1-\tau \text { a.s., } \quad \mathbb{E}\left[\mid V_{n, s}(l) \| \Omega_{n}\right]=O(1) .
$$

Proof. See Appendix E.

Loosely speaking, Proposition 1 states that, for both choices of $\alpha_{n}$, all but $\tau$ of $s$ 's PPR concentrates on a small neighborhood surrounding $s$, for any $\tau>0$. The difference is the size of this neighborhood: when $\alpha_{n}=\Theta(1 / \log n)$, the neighborhood grows with the graph; when $\alpha_{n}$ is constant, the neighborhood has constant size. From the PPR interpretation of Section 2.2, this suggests that the number of nodes that are important to $s$ grows in the former case but remains fixed in the latter case. We believe the former case is more appropriate. Additionally, the growth of this important set of nodes remains sublinear in $n$ in the former case; intuitively, this says that a vanishing fraction of all nodes are important to $s$, i.e. a notion of $s$ 's perspective remains. Later, we will further motivate this choice in terms of the mixing time of the simple random walk on $G_{n}$ (see Section 7.4). We also remark that, since PPR concentrates on a small neighborhood for this choice of $\alpha_{n}$, PPR vectors can be well-approximated by sparse estimates (with the sparsity precisely controlled by $\tau$ and $\rho$ ), which has implications in terms of both time and space complexity for algorithms we discuss in Section 5.1. Finally, we reiterate that for the remainder of the paper, we set

$$
\alpha_{n}=\frac{\rho \log (1 / \tau) \log (\zeta)}{\log n}=\Theta\left(\frac{1}{\log n}\right) .
$$




\subsection{Main result}

Before presenting our main result, we state the following tail bound for the event $B_{s}\left(K_{n}, \epsilon\right)$ (recall this event, defined in (3), states that $\pi_{s}$ is more than $\epsilon$ from a linear combination of $\left\{\pi_{k}\right\}_{k \in K_{n}}$ ). Our main result will follow almost immediately from this lemma. The bound is $n^{-\min \left\{c_{1}, c_{2} \epsilon^{2}\right\}}$ for constants $c_{i}$ depending only on the degree sequence and the choice of $\alpha_{n}$; hence, somewhat surprisingly, $\epsilon$ only affects the bound when it is sufficiently small.

Lemma 1. Given Assumption 1, we have for $s \sim V_{n}$ uniformly and for any $\epsilon>0$ independent of $n$,

$$
\mathbb{P}\left[B_{s}\left(K_{n}, \epsilon\right) \mid U_{s}=1\right]=O\left(n^{-c(\epsilon)}\right),
$$

where, with $\delta, p, \zeta$ from Assumption 1, and with $\rho, \tau$ from (5),

$$
c(\epsilon) \triangleq \min \left\{\delta, \frac{\log (1 / p)}{2 \log (\zeta / p)}, \frac{((1-p) \epsilon)^{2}}{2 \rho \log (1 / \tau) \log \zeta}\right\}>0 .
$$

The proof of Lemma 1 is lengthy and occupies Appendices A and D. For now, we note that the proof broadly requires four steps:

(1) Show that, for a certain choice of $\left\{\beta_{s}(k)\right\}_{k \in K_{n}}$, the error term $\left\|\pi_{s}-\left(\alpha_{n} e_{s}^{\top}+\sum_{k \in K_{n}} \beta_{s}(k) \pi_{k}\right)\right\|_{1}$ in $B_{s}\left(K_{n}, \epsilon\right)$ can be bounded by only examining the $m$-step neighborhood out of $s$. (Here the choice of $\left\{\beta_{s}(k)\right\}_{k \in K_{n}}$ arises from an alternate form of the Hubs Theorem from [33], which requires a new proof; the error bound is new, to the best of our knowledge.)

(2) Argue that, conditioned on certain events not occurring during the first $m$ steps of the graph construction, this error bound follows the same distribution as a quantity defined in terms of the first $m$ generations of a branching process. (Here we essentially argue that, before these events occur, a bijection exists between the subgraph that determines the error bound and the tree resulting from the branching process.)

(3) Bound the probability of these events occurring during the first $m$ iterations. (Here we use a modified version of Lemma 5.4 from [21]. Our modification weakens the assumptions of [21], allowing us to apply it to a wider class of degree sequences; see Section 7.3 for an example.)

(4) Bound the probability of $B_{s}\left(K_{n}, \epsilon\right)$, conditioned on these events not occurring, by analyzing the branching process quantity. (Here our analysis leverages the fact that the branching process quantity has a martingale-like structure.)

Before proceeding, we pause to state the choice of $\left\{\beta_{s}(k)\right\}_{k \in K_{n}}$ from Step 1, which will be used in Section 5. First, for any realization of the DCM and for $v \in V_{n} \backslash K_{n}$, we define

$$
\tilde{P}(i, j)=U_{i} P(i, j), \quad \tilde{P}_{v}=\left(1-\alpha_{n}\right) \tilde{P}+\left(\alpha_{n} e_{V_{n} \backslash K_{n}}+e_{K_{n}}\right) e_{v}^{\top},
$$

where $P$ is the normalized adjacency matrix from Section 2.2. Note $\tilde{P}_{v}$ corresponds to a Markov chain similar to that in Definition 1; however, upon reaching $K_{n}$, the random walker jumps back to $v$ with probability 1 (instead of $\alpha_{n}$ ). Letting $\tilde{\pi}_{v}$ denote the stationary distribution of this chain, one can show (see Appendix A.1)

$$
\pi_{v}(w)=\frac{\alpha_{n} U_{w} \tilde{\pi}_{v}(w)+\sum_{k \in K_{n}} \tilde{\pi}_{v}(k) \pi_{k}(w)}{\alpha_{n}+\left(1-\alpha_{n}\right) \tilde{\pi}_{v}\left(K_{n}\right)} \forall w \in V_{n}
$$

With (7) in mind, we define

$$
\beta_{v}(k)=\frac{\tilde{\pi}_{v}(k)}{\alpha_{n}+\left(1-\alpha_{n}\right) \tilde{\pi}_{v}\left(K_{n}\right)} \forall k \in K_{n},
$$

and we take $\left\{\beta_{s}(k)\right\}_{k \in K_{n}}$ as in (8) in Step 1 above.

We now turn to the main result, Theorem 1. Together with Assumption 2, it essentially states the following: when certain moments of the degree sequence exist, and when a sublinear number 
of nodes contains a constant fraction of instubs, the dimension of the set of PPR vectors scales sublinearly in $n$ (with high probability).

Theorem 1. Given Assumption 2, for any $\epsilon>0$ independent of $n$,

$$
\mathbb{E}\left[\Delta\left(K_{n}, \epsilon\right)\right]=O\left(n^{\max \{\kappa, 1-c(\epsilon)\}}\right),
$$

where $c(\epsilon)$ is defined in Lemma 1. Hence, $\forall \bar{c} \in(\max \{\kappa, 1-c(\epsilon)\}, 1)$ and $C>0$, both independent of $n$,

$$
\mathbb{P}\left[\Delta\left(K_{n}, \epsilon\right) \geq C n^{\bar{c}}\right]=O\left(n^{\max \{\kappa, 1-c(\epsilon)\}-\bar{c}}\right) \underset{n \rightarrow \infty}{\longrightarrow} 0 .
$$

Proof. See Appendix F.

\section{ALGORITHMIC CONSEQUENCES}

Having stated our structural result, Theorem 1, we next consider its algorithmic consequences. Broadly speaking, the main consequence is that only $\Delta\left(K_{n}, \epsilon\right)$ PPR vectors - those corresponding to $K_{n}$ and $\left\{v \in V_{n} \backslash K_{n}: B_{v}\left(K_{n}, \epsilon\right)\right.$ holds $\}$ - need be computed, after which the others can be estimated as linear combinations of $\left\{\pi_{k}\right\}_{k \in K_{n}}$ using the weights $\left\{\tilde{\pi}_{v}(k)\right\}_{k \in K_{n}}$. Because $\Delta\left(K_{n}, \epsilon\right)$ scales sublinearly per Theorem 1 , this may yield an order reduction when compared to the naive scheme of computing all $n$ PPR vectors. However, computing $\Delta\left(K_{n}, \epsilon\right)$ vectors and $n-\Delta\left(K_{n}, \epsilon\right)$ sets of weights $\left\{\tilde{\pi}_{v}(k)\right\}_{k \in K_{n}}$ remains too costly, so each of these quantities will be estimated. This introduces a nontrivial challenge: the errors incurred by estimating $\left\{\pi_{k}\right\}_{k \in K_{n}}$ and $\left\{\tilde{\pi}_{v}(k)\right\}_{k \in K_{n}}$ will propagate through to the estimate of $\pi_{v}$, potentially rendering it highly inaccurate. In this section, we devise and analyze a (low complexity) algorithm that overcomes this challenge. We then discuss how this algorithm relates to existing PPR estimation algorithms.

\subsection{Algorithm to estimate $\left\{\pi_{v}\right\}_{v \in V_{n}}$}

At a high level, our algorithm proceeds as follows. First, estimate $\left\{\pi_{k}\right\}_{k \in K_{n}}$ as $\left\{\hat{\pi}_{k}\right\}_{k \in K_{n}}$. Next, for $v \in V_{n} \backslash K_{n}$, estimate $\pi_{v}$ as

$$
\alpha_{n} e_{v}^{\top}+\frac{\sum_{k \in K_{n}} \hat{\tilde{\pi}}_{v}(k) \hat{\pi}_{k}}{\alpha_{n}+\left(1-\alpha_{n}\right) \hat{\tilde{\pi}}_{v}\left(K_{n}\right)},
$$

where $\hat{\tilde{\pi}}_{v}(k)$ is an estimate of $\tilde{\pi}_{v}(k)$. The basic idea behind this scheme is that, by (7), the estimate shown in (9) may be close to $\pi_{v}$. Throughout this section, we make this idea rigorous, developing an algorithm based on this idea and using Theorem 1 to analyze it.

The first step of our scheme is to estimate $\left\{\pi_{k}\right\}_{k \in K_{n}}$. Here we will use a modified version of the Approx-PageRank algorithm from [4], which we include as Algorithm 2 here. In particular, we have modified the original version to allow for the fact that our DCM is a multi-graph in general. Since we have modified Approx-PageRank, we include a modified version of the analysis from [4] in Appendix G. Specifically, we show that for any realization of $G_{n}$ and any $v \in V_{n}$, Approx-PageRank $\left(v, \epsilon_{1}\right)$ has complexity $O\left(n \log n / \epsilon_{1}\right)$, assuming $L_{n}=O(n)$ (as in Assumption 1$)$ and $\alpha_{n}=\Theta(1 / \log n)$. Hence, running Approx-PageRank $\left(k, \epsilon_{1}\right) \forall k \in K_{n}$ will yield estimates of $\left\{\pi_{k}\right\}_{k \in K_{n}}$ with error guarantees in the $l_{1}$ norm. We note the Approx-PageRank algorithm is essentially a refined version of the power iteration suggested by (4): while one could compute a finite number of the vector-matrix products in (4), the $i$-th of which corresponds to $i$-step probabilities out of $v$, Approx-PageRank only computes high-probability paths that contribute the most to $\pi_{v}$.

We next consider estimation of $\left\{\tilde{\pi}_{v}(k)\right\}_{v \in V_{n} \backslash K_{n}, k \in K_{n}}$; here we desire an $l_{\infty}$ error guarantee that, paired with the $l_{1}$ guarantee on $\hat{\pi}_{k}$, will yield an $l_{1}$ guarantee on (9). A natural algorithm to consider is the Approx-Contributions algorithm from [3], which, given $v \in V_{n}$, estimates $\left\{\pi_{u}(v)\right\}_{u \in V_{n}}$ with an $l_{\infty}$ guarantee. However, since we seek estimates of $\left\{\tilde{\pi}_{v}(k)\right\}_{v \in V_{n} \backslash K_{n}}$, not $\left\{\pi_{v}(k)\right\}_{v \in V_{n} \backslash K_{n}}$, 
the existing algorithm does not directly apply. For this reason, we will consider a modified version of Approx-Contributions. This is based on our analysis in Appendix D.2, which shows (see (56))

$$
\tilde{\pi}_{v}(k)=\frac{\alpha_{n} \mu_{v}(k)}{1-\left(1-\alpha_{n}\right) \mu_{v}\left(K_{n}\right)} \forall v \in V_{n} \backslash K_{n}, k \in K_{n},
$$

where $\mu_{v}(k)$ is the $k$-th element of $\mu_{v}=e_{v}^{\top}\left(I-\left(1-\alpha_{n}\right) \tilde{P}\right)^{-1}$ (here $\tilde{P}$, defined in (6), is the rownormalized adjacency matrix with rows corresponding to $K_{n}$ set to zero). Note that $\mu_{v}$ has nearly the same form as $\pi_{v}$, by (4); in particular, $\mu_{v}$ is a scaled PPR vector defined on a modified graph. Hence, we will use a modified version of Approx-Contributions to estimate $\left\{\mu_{v}(k)\right\}_{v \in V_{n}}$ for each $k \in K_{n}$, after which we may estimate $\left\{\tilde{\pi}_{v}(k)\right\}_{v \in V_{n} \backslash K_{n}}$ as

$$
\hat{\tilde{\pi}}_{v}(k)=\frac{\alpha_{n} \hat{\mu}_{v}(k)}{1-\left(1-\alpha_{n}\right) \hat{\mu}_{v}\left(K_{n}\right)} \forall v \in V_{n} \backslash K_{n}, k \in K_{n} .
$$

This modification is necessary to account for the different scaling between $\pi_{v}$ and $\mu_{v}$, as well as the fact that $G_{n}$ may be a multi-graph in our setting. With this in mind, we define a modified version of Approx-Contributions in Algorithm 3; we include an analysis in Appendix G, based on [3], which includes our desired bound on $\left|\tilde{\pi}_{v}(k)-\hat{\tilde{\pi}}_{v}(k)\right|$. We note that Approx-Contributions can be interpreted as the dual of Approx-PageRank: as discussed above, the latter computes highprobability paths out of $v$; analogously, the former computes high-probability paths into $v$.

After computing $\left\{\hat{\pi}_{k}\right\}_{k \in K_{n}}$ and $\left\{\hat{\tilde{\pi}}_{v}(k)\right\}_{v \in V_{n} \backslash K_{n}, k \in K_{n}}$ via Algorithms 2 and 3, we could immediately compute (9) and return this as our estimate of $\pi_{v}$ for $v \in V_{n} \backslash K_{n}$. There are two drawbacks to this approach, both of which our algorithm will address. The first drawback is that, while Lemma 1 guarantees $\pi_{v}$ is close to

$$
\alpha_{n} e_{v}^{\top}+\frac{\sum_{k \in K_{n}} \tilde{\pi}_{v}(k) \pi_{k}}{\alpha_{n}+\left(1-\alpha_{n}\right) \tilde{\pi}_{v}\left(K_{n}\right)}
$$

for most $v$ (namely, all but a sublinear number), it could be far from (10) for some $v$; hence, the estimate given by (9) may be inaccurate for some $v$ as well. The second drawback is that computing (9) for every $v \in V_{n} \backslash K_{n}, k \in K_{n}$ requires matrix multiplication that has complexity $O\left(n^{2+\kappa}\right)$ when $\left|K_{n}\right|=O\left(n^{\kappa}\right)$; as we discuss in Section 5.2, existing methods can estimate $\left\{\pi_{v}\right\}_{v \in V_{n}}$ in our setting with complexity $O\left(n^{2} \log n\right)$ and with the same accuracy guarantee we will provide. Hence, we next address each of these drawbacks.

First, to address the accuracy concern, we show in Appendix G that $\hat{\tilde{\pi}}_{v}\left(K_{n}\right)$ can be used at runtime to determine whether (9) will be sufficiently accurate, without actually computing (9) or knowing $\pi_{v}$ itself. Specifically, we show that if $\hat{\tilde{\pi}}_{v}\left(K_{n}\right)$ exceeds $g_{n}\left(\epsilon_{1}\right)$, where $\epsilon_{1}>0$ and

$$
g_{n}\left(\epsilon_{1}\right)=\frac{\alpha_{n}\left(1-\left(\epsilon_{1}+\alpha_{n}\right)\right)}{\epsilon_{1}+\alpha_{n}\left(2-\left(\epsilon_{1}+\alpha_{n}\right)\right)}
$$

then (9) will be close to $\pi_{v}$ in the $l_{1}$ norm. Note that the condition $\hat{\tilde{\pi}}_{v}\left(K_{n}\right) \geq g_{n}\left(\epsilon_{1}\right)$ intuitively states that $K_{n}$ is "close" to $v$ in the graph; hence, the preceding argument essentially says (9) is a good estimate of $\pi_{v}$ whenever $K_{n}$ is close to $v$, which is an intuitive condition. ${ }^{4}$

\footnotetext{
$\overline{ }^{4}$ The form of $g_{n}(\cdot)$ can be further explained by two special cases. First, observe $g_{n}\left(1-\alpha_{n}\right)=0 \leq \hat{\tilde{\pi}}_{v}\left(K_{n}\right)$, i.e. $\pi_{v}$ is always within $l_{1}$ distance $\left(1-\alpha_{n}\right)$ of $(10)$; this is in fact proven in (53) in Appendix D.2. Second, $g_{n}(0)=\left(1-\alpha_{n}\right) /\left(2-\alpha_{n}\right)$, i.e. the error is zero when $\hat{\tilde{\pi}}_{v}\left(K_{n}\right) \geq\left(1-\alpha_{n}\right) /\left(2-\alpha_{n}\right)$. This corresponds to the case $N_{\text {out }}(v) \subset K_{n}$, where $N_{\text {out }}(v)$ are the outgoing neighbors of $v$, i.e. $K_{n}$ is "maximally close" to $v$. Indeed, by $\tilde{\pi}_{v}=\tilde{\pi}_{v} \tilde{P}_{v}$ one can verify that if $N_{o u t}(v) \subset K_{n}$,

$$
\tilde{\pi}_{v}(v)=\frac{1}{2-\alpha_{n}}, \quad \tilde{\pi}_{v}(u)=\frac{1-\alpha_{n}}{D_{v}\left(2-\alpha_{n}\right)} \forall u \in N_{\text {out }}(v), \quad \tilde{\pi}_{v}(u)=0 \forall u \in V_{n} \backslash\left(N_{\text {out }}(v) \cup\{v\}\right),
$$

so that $\tilde{\pi}_{v}\left(K_{n}\right)=\tilde{\pi}_{v}\left(N_{\text {out }}(v)\right)=\left(1-\alpha_{n}\right) /\left(2-\alpha_{n}\right)$.
} 
Second, to address the matrix multiplication concern, we will "sparsify" the matrix $\left\{\hat{\pi}_{k}\right\}_{k \in K_{n}}$, i.e. set certain elements of this matrix to zero. We will do so in a manner that ensures (1) enough elements are set to zero to guarantee the resulting multiplication has complexity $o\left(n^{2}\right)$, and (2) not enough elements are set to zero to significantly alter the accuracy of the resulting estimates. In particular, we will set $\hat{\pi}_{k}(u)$ to zero whenever $u \notin V_{n, k}(l)$, where $l$ is an input to our algorithm and $V_{n, k}(l)$ is the $l$-step neighborhood out of $k$ (as in Proposition 1). Using an argument similar to the proof of Proposition 1, we will show that $\left|V_{n, k}(l)\right|$ scales sublinearly in $n$ (which will address point (1) above) and that $\hat{\pi}_{k}(u)$ is small whenever $u \notin V_{n, k}(l)$ (which will address point (2) above).

Having described each step of our scheme, we provide a formal definition in Algorithm 1. Theorem 2 provides two guarantees for Algorithm 1, which can be stated informally as follows:

(1) For certain choices of $\tau$ in the definition of $\alpha_{n}$, each estimate $\hat{\pi}_{v}$ is accurate in the $l_{1}$ norm a.s. In fact, this guarantee holds for any underlying graph $G_{n}$ (not just the DCM).

(2) For certain choices of $\rho$ in the definition of $\alpha_{n}$, the complexity is $o\left(n^{2}\right)$ with high probability, assuming Assumption 2 holds and each $k \in K_{n}$ has $O(1)$ out-degree. (Note this strengthens the previous assumption of $O(1)$ average out-degree.)

We reemphasize that Algorithm 1 generates estimates accurate in the $l_{1}$ norm for any graph $G_{n}$. We also note that an $l_{1}$ guarantee is a natural objective, since bounding $l_{1}$ distance is equivalent to bounding total variation distance, total variation is a standard distance for comparing distributions, and PPR vectors are distributions over $V_{n}$. Additionally, while the complexity guarantee pertains to a class of graphs, we suspect the algorithm will perform well for a wider class of graphs. For example, we believe that graphs with in-degrees following a power law satisfy the most crucial of our assumptions (see Section 7.2). Hence, while the entirety of Theorem 2 applies to a class of graphs, we believe Algorithm 1 is of wider value.

Theorem 2. Let $\epsilon \in(0,1)$ be a constant, and set

$$
\epsilon_{1}=\frac{\epsilon}{4}, \quad \epsilon_{2}=\frac{\alpha_{n}^{2} g_{n}(\epsilon / 4)}{2\left|K_{n}\right|}, \quad l=\left\lceil\frac{\log (1 / \tau)}{\alpha_{n}}\right\rceil
$$

in Algorithm 1. Then the following hold:

- (Accuracy) Assume $\tau \leq \epsilon / 4$ in the definition of $\alpha_{n}$. Then for an arbitrary graph $G_{n}$,

$$
\left\|\hat{\pi}_{v}-\pi_{v}\right\|_{1}<\epsilon \forall v \in V_{n} \text { a.s. }
$$

- (Complexity) Assume $G_{n}$ is the DCM and the following hold:

- Assumption 2.

- $\rho>\frac{1}{1-\kappa}$ in the definition of $\alpha_{n}$, with $\kappa$ from Assumption 2.

- There exist constants $\delta^{\prime}>\kappa$ and $D_{\max }>0$ such that

$$
\mathbb{P}\left[\max _{k \in K_{n}} D_{k}>D_{\max } \mid \Omega_{n}\right]=O\left(n^{-\delta^{\prime}}\right) .
$$

Then, letting $C_{A l g 1}$ denote the complexity of Algorithm 1,

$$
\mathbb{E}\left[C_{A l g 1} \mid \Omega_{n}\right]=O\left(\max \left\{\mathbb{E}\left[\Delta\left(K_{n}, \epsilon / 14\right)\right] n(\log n)^{3}, n^{\max \left\{1+\kappa+1 / \rho, 2+\kappa-\delta^{\prime}\right\}}\right\}\right) .
$$

Furthermore, $\exists \bar{c}<2$ such that

$$
\lim _{n \rightarrow \infty} \mathbb{P}\left[C_{A l g 1} \geq n^{\bar{c}} \mid \Omega_{n}\right]=0 .
$$

Proof. See Appendix G. 

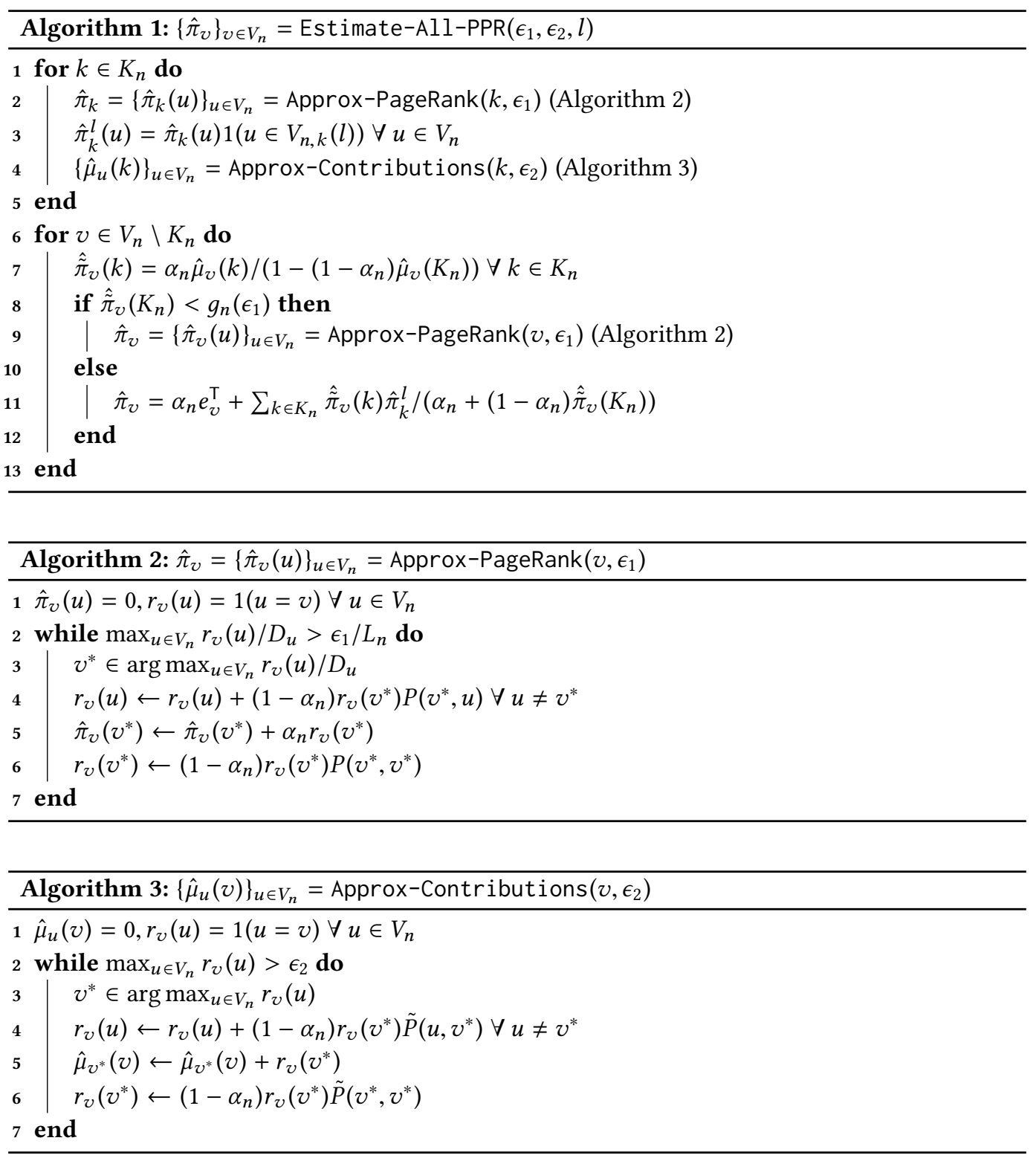

We note that the $\mathbb{E}\left[\Delta\left(K_{n}, \epsilon / 14\right) n(\log n)^{3}\right]$ term in the complexity guarantee accounts for all but Line 11 of Algorithm 1. Roughly speaking, the proof shows that Approx-PageRank need only be run $\Delta\left(K_{n}, \epsilon / 14\right)$ times, once for each node belonging to $K_{n}$ or $\left\{v \in V_{n} \backslash K_{n}: B_{v}\left(K_{n}, \epsilon / 14\right)\right.$ holds $\}$; for other nodes $v, \pi_{v}$ can be well-approximated as a linear combination of $\left\{\hat{\pi}_{k}\right\}_{k \in K_{n}}$. The proof also shows that each run of Approx-PageRank has complexity linear in $n$; hence, if instead $\Delta\left(K_{n}, \epsilon / 14\right)$ scaled linearly in $n$, Algorithm 1 would have complexity quadratic in $n$. Because of this, Theorem 2 can be viewed as a consequence of Theorem 1. (We note $\Delta\left(K_{n}, \epsilon / 14\right)$ appears because the if statement in Algorithm 1 relies on an estimate of $\tilde{\pi}_{v}\left(K_{n}\right)$, not $\tilde{\pi}_{v}\left(K_{n}\right)$ itself; if $\tilde{\pi}_{v}\left(K_{n}\right)$ were known, $\Delta\left(K_{n}, \epsilon\right)$, a smaller quantity, would instead appear. The factor 14 has no particular significance.) On 
the other hand, the $n^{\max \left\{1+\kappa+1 / \rho, 2+\kappa-\delta^{\prime}\right\}}$ term in the complexity guarantee accounts for the matrix multiplication in Line 11 and requires the additional assumptions stated in Theorem 2.

One issue we have not addressed is how to choose $K_{n}$ to guarantee low complexity; our analysis simply says if an appropriate $K_{n}$ exists, then the algorithm has subquadratic complexity. We believe choosing nodes of highest in-degree as $K_{n}$ is a good choice; see Section 6 for empirical results and Section 7.1 for some theoretical evidence. However, an important (though, we believe, a very difficult) question for future work is as follows: given $\epsilon>0$, how can one optimally choose $K_{n}=K_{n}(\epsilon)$ to ensure $\epsilon$-accuracy while minimizing complexity in Algorithm 1?

Finally, we note Algorithm 1 can be easily modified to obtain a variant that uses precomputation and that proceeds as follows:

- Offline stage: Run Lines 1-5 of Algorithm 1; store $\left\{\hat{\pi}_{k}^{l}\right\}_{k \in K_{n}}$ and $\left\{\hat{\mu}_{k}(k)\right\}_{u \in V_{n}, k \in K_{n}}$.

- Online stage: When an estimate of $\pi_{k}, k \in K_{n}$ is needed, return $\hat{\pi}_{k}^{l}$; when an estimate of $\pi_{v}, v \notin K_{n}$ is needed, run Lines 7-12 of Algorithm 1 .

In Appendix G.3, we show that, under the assumptions of Theorem 2, the offline stage requires $O\left(n^{1+\kappa}\right)$ storage, the estimate returned during the online stage has $l_{1}$ error bounded by $\epsilon$, and for $s \sim V_{n}$ uniformly, the online stage has complexity

$$
O\left(\max \left\{\Delta\left(K_{n}, \epsilon / 14\right) \log n, n^{\kappa+\max \left\{1 / \rho, 1-\delta^{\prime}\right\}}\right\}\right) .
$$

Note that this storage is subquadratic, strictly better than the $n^{2}$ storage required the store the PPR matrix itself. Additionally, with $\kappa<\max \left\{\delta^{\prime}, 1-1 / \rho\right\}$ per the assumptions of Theorem 2 , the online stage has sublinear complexity, strictly better than the existing approach of running Approx-PageRank for $s \sim V_{n}$ uniformly online $(O(n \log n)$ complexity).

\subsection{Comparison to other algorithms}

In the previous section, we showed that Algorithm 1 estimates $\left\{\pi_{v}\right\}_{v \in V_{n}}$ with constant error in the $l_{1}$ norm for each vector and has complexity $o\left(n^{2}\right)$ on the DCM (under appropriate assumptions). To the best of our knowledge, this complexity bound is strictly better than any in the literature. In fact, the best existing algorithm is to simply run Approx-PageRank $(v, \epsilon) \forall v \in V_{n}$, which, by Lemma 12 in Appendix G, guarantees constant $l_{1}$ error and has complexity $O\left(n^{2} \log n\right)$ when $L_{n}=O(n), \alpha_{n}=\Theta(1 / \log n)$ (i.e. in the setting considered in Theorem 2). Alternatively, the original version of Approx-Contributions from [3] guarantees $l_{\infty}$ error bounded by $\epsilon_{n}$ with complexity $O\left(L_{n} /\left(n \alpha_{n} \epsilon_{n}\right)\right)$ for a uniformly random node (see Theorem 2 in [40]). Hence, running this algorithm for all nodes, setting $\epsilon_{n}=\epsilon / n$ to obtain constant $l_{1}$ error, and taking $L_{n}=O(n), \alpha_{n}=\Theta(1 / \log n)$ as in our setting, this complexity is also $O\left(n^{2} \log n\right)$. We do concede that these $O\left(n^{2} \log n\right)$ bounds only require $L_{n}=O(n)$, while ours pertains to a specific class of random graphs; however, we believe this class contains reasonable models for many graphs of interest (see Section 7.3).

We also note that we have conditioned on $\Omega_{n}$ for the complexity guarantee of Theorem 2 because, if instead $\Omega_{n}^{C}$ holds, we could have $\Delta\left(K_{n}, \epsilon / 14\right)=O(n), L_{n}=O\left(n^{2}\right)$, in which case Algorithm 1 will have complexity $O\left(n^{3} \log n\right)$, the same as the existing methods Approx-PageRank and Approx-Contributions. However, if we assume $L_{n}=O(n)$ with probability 1 , we can write

$$
\mathbb{E}\left[C_{A l g 1}\right] \leq \mathbb{E}\left[C_{A l g 1} \mid \Omega_{n}\right]+O\left(n^{2} \log n\right) \mathbb{P}\left[\Omega_{n}^{C}\right]=o\left(n^{2}\right),
$$

where the first term is $o\left(n^{2}\right)$ by Theorem 2 and the second is $o\left(n^{2}\right)$ since $\mathbb{P}\left[\Omega_{n}^{C}\right]=O\left(n^{-\delta}\right), \delta>0$ by Assumption 1. In other words, when $L_{n}=O(n)$ with probability 1 , Algorithm 1 is subquadratic and thus strictly better than existing methods without conditioning on $\Omega_{n}$.

As mentioned in Sections 1 and 3, our algorithm is similar to the one proposed, but not analyzed, in [33]. This scheme follows the high-level description from the beginning of Section 5.1: first, estimate 
$\left\{\pi_{k}\right\}_{k \in K_{n}}$ and $\left\{\tilde{\pi}_{v}(k)\right\}_{v \in V_{n} \backslash K_{n}, k \in K_{n}}$; second, for $v \notin K_{n}$, approximate $\pi_{v}$ as a linear combination via (9). The estimation in the first step uses dynamic programming (DP) algorithms similar in spirit to the methods Approx-PageRank and Approx-Contributions that we use. However, no analysis is provided (in terms of accuracy or complexity) for these DP algorithms. Moreover, even if guarantees did exist for these DP algorithms, non-trivial subtleties arise when using such guarantees to analyze the overall estimation scheme, as was seen in Section 5.1; these subtleties were not addressed in [33] either. In contrast, our algorithm and its analysis provide the type of guarantees one would desire in practice: an accuracy guarantee for all estimates, a complexity guarantee for certain choices of $K_{n}$, and evidence for why choosing $K_{n}$ as nodes of high in-degree is a good choice.

Another existing work worth mentioning is [18]. Here the authors provide an algorithm to estimate $\pi_{v}$ such that

$$
\left(1-\epsilon^{\prime}\right) \pi_{v}(u)-\epsilon_{n} \leq \hat{\pi}_{v}(u) \leq\left(1+\epsilon^{\prime}\right) \pi_{v}(u)+\epsilon_{n} \forall u \in V_{n} .
$$

The complexity of this scheme is $O\left((\log n)^{2} \log \left(1 / \epsilon_{n}\right) /\left(\epsilon_{n}\left(\epsilon^{\prime}\right)^{2}\right)\right)$ per node. Hence, setting $\epsilon_{n}=$ $\epsilon / n$ for some constant $\epsilon$, setting $\epsilon^{\prime}$ small and independent of $n$, and running this scheme for all nodes gives complexity $O\left(n^{2}(\log n)^{3}\right)$, similar to Approx-PageRank and Approx-Contributions. However, the multiplicative error tolerance term $\epsilon^{\prime}$ cannot be avoided, so this is not quite an $l_{1}$ error guarantee. The analysis in [18] also shows via a tight lower bound that the complexity bound is within a polylogarithmic factor of the optimal. Naively using their lower bound independently for each node would lead to the erroneous claim that any algorithm for estimating all the PPR vectors should have complexity at least $\Omega\left(n^{2}\right)$. Critically, though, properly accounting for the dependence of the PPR vectors based on the common underlying graph allows us to do better than this naive conjectured lower bound.

We also believe our analysis can used to obtain tighter complexity bounds for modified versions of other algorithms, similar to the analysis we have conducted here for a modified version of the algorithm from [33]. For example, [39] provides a scheme to estimate $\pi_{v}(u)$ with sublinear complexity $O(\sqrt{n} \log n)$ in the $L_{n}=O(n), \alpha_{n}=\Theta(1 / \log n)$ setting, and with accuracy guarantee

$$
\left|\hat{\pi}_{v}(u)-\pi_{v}(u)\right| \leq\left\{\begin{array}{ll}
\epsilon \pi_{v}(u), & \pi_{v}(u) \geq 1 / n \\
2 e / n, & \pi_{v}(u)<1 / n
\end{array},\right.
$$

i.e. a relative error bound when $\pi_{v}(u)$ is large and an absolute error guarantee otherwise. If one desires this accuracy guarantee for the entire matrix $\left\{\pi_{v}(u)\right\}_{v, u \in V_{n}}$, the scheme can be run separately for every $v, u$ pair at complexity $O\left(n^{2.5} \log n\right)$ (which is strictly better than computing the matrix via the inverse in (4)). However, the basic approach we have used here could also be used to reduce this complexity; namely, by first using the scheme to estimate $\left\{\pi_{k}\right\}_{k \in K_{n}}$ and $\left\{\tilde{\pi}_{v}(k)\right\}_{k \in K_{n}}$ and then using (9) to estimate $\left\{\pi_{v}(u)\right\}_{v, u \in V_{n} \backslash K_{n}}$. The challenge of designing such an algorithm would be similar to the challenge we encountered in this section. Specifically, we had to carefully design certain aspects of Algorithm 1 - "sparsifying" the matrix $\left\{\hat{\pi}_{k}\right\}_{k \in K_{n}}$ and checking if $\hat{\tilde{\pi}}_{v}\left(K_{n}\right)$ exceeds $g_{n}\left(\epsilon_{1}\right)$ - to ensure that estimation errors pertaining to $\left\{\pi_{k}\right\}_{k \in K_{n}}$ and $\left\{\tilde{\pi}_{v}(k)\right\}_{k \in K_{n}}$ did not propagate through to estimates of $\left\{\pi_{v}(u)\right\}_{v, u \in V_{n} \backslash K_{n}}$ and render them highly inaccurate. Similarly, using our framework to estimate the PPR matrix using the algorithm of [39] would require a careful analysis of how the error given by (11) propagates through to later estimates. To summarize, we believe our basic approach can be used to design modified versions of other existing algorithms; however, we suspect this will be nontrivial, suggesting an avenue for future work.

As mentioned in Section 2.3, our algorithm also bears resemblance to nonnegative matrix factorization (NMF), which, given $X \in \mathbb{R}^{n \times m}$, seeks $W \in \mathbb{R}^{n \times r}, H \in \mathbb{R}^{r \times m}$ such that $\|X-W H\|$ is small (where typically $r \ll m, n$ to guarantee a low-rank estimate). This is directly analogous to 
our algorithm. Indeed, Algorithm 2 generates $W \in \mathbb{R}^{n \times \Delta\left(K_{n}, \epsilon / 14\right)}, H \in \mathbb{R}^{\Delta\left(K_{n}, \epsilon / 14\right) \times n}$ satisfying

$$
\left\|\left(\Pi_{n}-\alpha_{n} I\right)-W H\right\|_{\infty}<\epsilon
$$

where $\|A\|_{\infty}=\max _{i}\left\|a_{i}\right\|_{1}$ for a matrix $A$ with rows $\left\{a_{i}\right\}$. However, there are some key differences between NMF and our scheme. First, NMF assumes $X$ is known, while in our algorithm $\Pi_{n}-\alpha_{n} I$ (which plays the role of $X$ ) is unknown. This means that standard NMF algorithms, which compute gradients dependent on $X$ to iteratively update $W, H$ (see e.g. [35]), do not apply. Additionally, computing these gradients requires the objective function $\|X-W H\|$ to be differentiable; in contrast, we use the non-differentiable norm $\|\cdot\|_{\infty}$, which further prohibits use of standard NMF algorithms. Finally, NMF chooses the dimensions of $W, H$ a priori, while our algorithm adjusts $r$ at runtime so as to minimize complexity (ultimately yielding $r=\Delta\left(K_{n}, \epsilon / 14\right)$ as above). In short, our algorithm can be viewed as a variant of NMF, tailored to the PPR setting in a manner that guarantees high accuracy and low complexity. We discuss this in more detail in Appendix C.1. We also that NMF with an objective function similar to ours has been studied in the contextual bandits literature. Namely, [44] analyzes a model for which $X_{i, j}$ gives the mean reward of pulling arm $i$ given context $j$. The authors propose a regret-minimization algorithm to minimize $\|X-W H\|_{\infty, \infty}$, where $\|A\|_{\infty, \infty}$ is the maximal element (in absolute value) of a matrix A. However, [44] assumes a small dimensionality by assuming a particular generative model for $W, H$; in contrast, our work develops conditions to prove a small dimensionality.

In light of this, we mention another general matrix problem of relevance; namely, low-rank approximation. For low-rank approximation, we are given a matrix $X \in \mathbb{R}^{n \times m}$ and aim to solve

$$
\inf _{\hat{X} \in \mathbb{R}^{n \times m}}\|X-\hat{X}\| \text { s.t. } \operatorname{rank}(\hat{X}) \leq r,
$$

for some $r \in\{1,2, \ldots, n\}$ (for example, when $\|\cdot\|$ is the spectral or Frobenius norm, the minimizing $\hat{X}$ is a truncated singular value decomposition of $X$, see e.g. Section 2.4 of [41]). A related problem, which can be viewed as the dual of low-rank approximation, instead aims to solve

$$
\min _{\hat{X} \in \mathbb{R}^{n \times m}} \operatorname{rank}(\hat{X}) \text { s.t. }\|X-\hat{X}\|<\epsilon .
$$

This is quite similar to our dimensionality measure. Indeed, (2) is a variant of (12) in which the minimum is taken over a restricted class of matrices (those containing a subset of rows of the original matrix, in addition to linear combinations of this subset). Hence, as in the above discussion of NMF, our scheme can be viewed as a tailored version of low-rank approximation.

Finally, we note that estimating the PPR matrix can be viewed as a special case of the algorithm from [30], which studies representation learning on graphs. However, we believe this algorithm will generally offer worse performance than ours in the PPR setting; see Appendix C.2.

\section{EXPERIMENTS}

In this section, we illustrate various aspects of our analysis with some empirical results. Our goal is to demonstrate that our theoretical findings - namely, that $\pi_{s}$ can be well-approximated as a linear combination of $\left\{\pi_{k}\right\}_{k \in K_{n}}$ (Lemma 1) and that PPR dimensionality is small (Theorem 1) - may still hold when our assumptions fail. To this end, we will estimate our dimensionality measure for two real graphs, as well as showing that $\pi_{s}$ is indeed well-approximated for a wider class of real graphs. Additionally, since our theoretical results are asymptotic statements, we will investigate the relevant quantities as $n$ grows for the DCM (using a variety of degree sequences). We note that, unless otherwise mentioned, we choose $K_{n}$ as the $n^{\kappa}$ nodes of highest in-degree, per the discussion of Section 7.1 (with $\kappa$ specified for each experiment). 


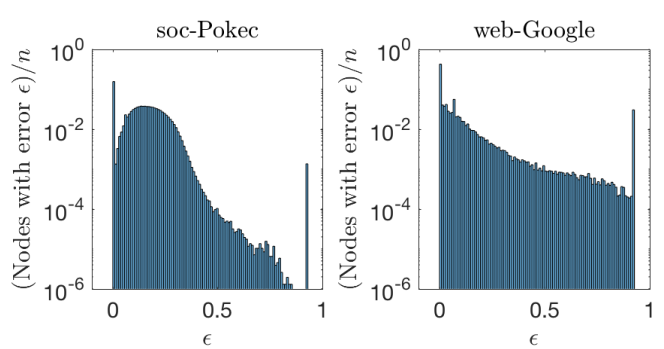

(a) Histograms of the error term (13)

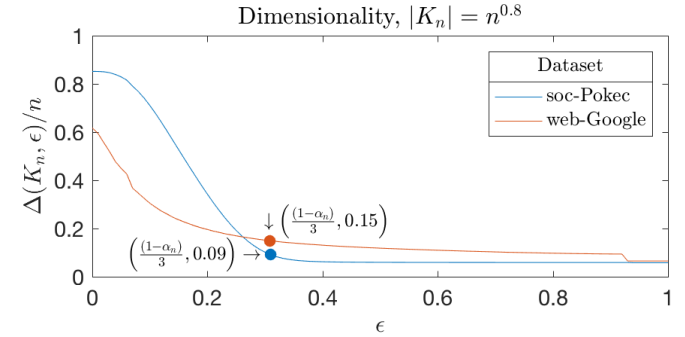

(b) $\Delta\left(K_{n}, \epsilon\right)$ is a small fraction of $n$ when $\epsilon=\frac{1-\alpha_{n}}{3}$ (ensuring estimation error is reduced by a factor of 3 )

Fig. 1. Error and dimensionality for soc-Pokec (a social network) and web-Google (a partial web graph).

We begin by estimating $\Delta\left(K_{n}, \epsilon\right)$ for a range of $\epsilon$ when $\kappa=0.8$ ( 0.8 was chosen as we found it roughly balanced the two summands in (2)). For this, we use two datasets from the Stanford Network Analysis Platform (SNAP) [37]: soc-Pokec, a social network, and web-Google, a partial web graph (see Appendix B.1 for details). We set $\alpha_{n}=1 / \log n$, and, $\forall v \notin K_{n}$, compute a bound on

$$
\left\|\pi_{v}-\left(\alpha_{n} e_{v}^{\top}+\frac{\sum_{k \in K_{n}} \tilde{\pi}_{v}(k) \pi_{k}}{\alpha_{n}+\left(1-\alpha_{n}\right) \tilde{\pi}_{v}\left(K_{n}\right)}\right)\right\|_{1}
$$

using a power iteration scheme described in Appendix B.2. Adding $n^{0.8}$ to the number of $v \in V_{n} \backslash K_{n}$ for which this bound exceeds $\epsilon$ then gives a bound on $\Delta\left(K_{n}, \epsilon\right)$. Figure 1a shows histograms of the error bound, while Figure $1 \mathrm{~b}$ shows our dimensionality measure. We highlight two points on Figure $1 \mathrm{~b},\left(\left(1-\alpha_{n}\right) / 3,0.09\right)$ for soc-Pokec and $\left(\left(1-\alpha_{n}\right) / 3,0.15\right)$ for web-Google. We believe $\left(1-\alpha_{n}\right) / 3$ is a reasonable choice of $\epsilon$ because (as proven in (53)) (13) is bounded by $1-\alpha_{n}$; hence, this choice of $\epsilon$ reduces the worst-case error term by a factor of 3 . We observe that $\Delta\left(K_{n},\left(1-\alpha_{n}\right) / 3\right)$ is small for both datasets ( $9 \%$ and $15 \%$ of nodes, respectively). This suggests two things. First, while Theorem 1 does not apply, the dimension of $\left\{\pi_{v}\right\}_{v \in V_{n}}$ appears small. Second, while Theorem 2 does not apply, $\left\{\pi_{v}\right\}_{v \in V_{n}}$ can be efficiently estimated by Algorithm 1.

We offer several other remarks on Figure 1. First, as proven in Appendix B.2, (13) is zero when $v \in V_{n, 0}$, where

$$
V_{n, 0}=\left\{v \notin K_{n}: \nexists\left(w, w^{\prime}\right) \in E_{n} \text { s.t. } w=v, w^{\prime} \notin K_{n}\right\} .
$$

(In words, the error is zero when no outgoing neighbors of $v$ belong to $V_{n} \backslash K_{n}$.) As a result, the "spikes" at $\epsilon=0$ in Figure 1a have height $\left|V_{n, 0}\right| / n$, and $\Delta\left(K_{n}, 0\right)=n-\left|V_{n, 0}\right|$ in Figure 1b. Next, the aforementioned claim that (13) is bounded by $1-\alpha_{n}$ explains the spikes at right in Figure 1a and the "dips" at right in Figure $1 \mathrm{~b}$ (both of which occur at $\epsilon=1-\alpha_{n}$ ). Finally, we observe that, between the spikes at $\epsilon=0$ and $\epsilon=1-\alpha_{n}$, the soc-Pokec histogram quickly decays beyond $\epsilon \approx 0.3$; this corresponds to the dimensionality being nearly flat beyond $\epsilon \approx 0.3$ in Figure $1 \mathrm{~b}$.

Computing (13) for every $v \in V_{n} \backslash K_{n}$ requires significant computational time, but (as described in Appendix B.2) we can also compute a bound on the average error

$$
\frac{1}{\left|V_{n} \backslash K_{n}\right|} \sum_{v \in V_{n} \backslash K_{n}}\left\|\pi_{v}-\left(\alpha_{n} e_{v}^{\top}+\frac{\sum_{k \in K_{n}} \tilde{\pi}_{v}(k) \pi_{k}}{\alpha_{n}+\left(1-\alpha_{n}\right) \tilde{\pi}_{v}\left(K_{n}\right)}\right)\right\|_{1}
$$




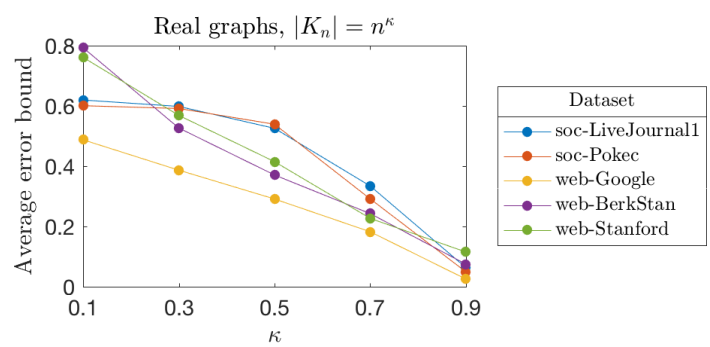

(a) Average error decreases as $\left|K_{n}\right|$ grows for a variety of social networks and web graphs.

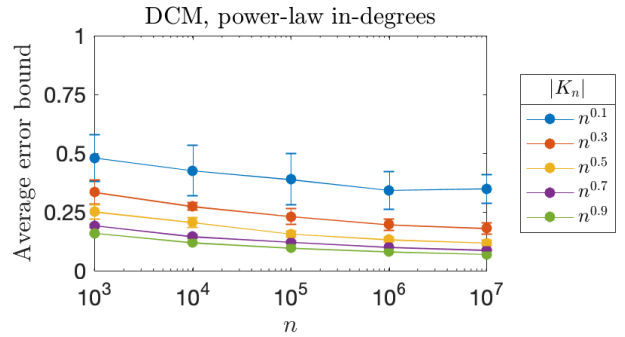

(b) For $s \sim V_{n} \backslash K_{n}$ uniformly on the DCM with power law in-degrees, error decreases as $n$ grows.

Fig. 2. Average error experiments for real and synthetic datasets

much more efficiently. Hence, we show this average error bound for a wider variety of SNAP datasets in Figure 2a, and for several $\kappa$ values. Interestingly, the social networks soc-LiveJournal1 and socPokec have similar behavior, as do the web graphs web-BerkStan and web-Stanford (web-Google is somewhat of an outlier; we believe this is in part because its $\left|V_{n, 0}\right|$ is largest).

We next replicate this average error experiment for two synthetic graphs, which allows us to observe how the bound on (15) evolves as $n$ grows. The first graph we consider is a DCM with power law in-degrees with exponent 2, i.e. $\mathbb{P}\left[N_{v}=i\right] \propto i^{-2} \forall v \in V_{n}, i \in\{1,2, \ldots, n\}$, and out-degrees generated as in Algorithm 4 from Section 7.3 (which, in expectation, gives constant out-degree to each node). We note this in-degree model is a common one for many graphs observed in practice (see Section 7.2 for details); however, Lemma 1 does not apply, as the in-degree sequence does not satisfy all of our assumptions (for instance, the expected in-degree does not converge). Nevertheless, Figure $2 \mathrm{~b}$ shows that the average error bound decays as $n$ grows across all choices of $\kappa$. We suspect this is in part because, while the degree sequence does not satisfy all of our assumptions, empirical results show that it contains a vanishing fraction of nodes with a non-vanishing fraction of edges (see Section 7.2). We believe this to be the most important of our assumptions.

Finally, we replicate the average error experiment with the sequence $\left(\mathrm{N}_{n}, \mathrm{D}_{n}, \mathrm{U}_{n}\right)$ generated via Algorithm 4 in Section 7.3; this sequence provably satisfies Assumption 2. We note that Algorithm 4 chooses $\left|K_{n}\right|$ in a manner that guarantees $\mathbb{E}\left[\left|K_{n}\right|\right]=n^{\kappa}$ (unlike previous experiments, for which $\left|K_{n}\right|=n^{\kappa}$ by design). Hence, in Figure 3a, we show the average error bound for a variety of $\mathbb{E}\left[\left|K_{n}\right|\right]$ choices. For choices of $\kappa$ at or above 0.5 , the average error bound slightly decays as $n$ grows (though less notably than in Figure $2 \mathrm{~b})$. We also conduct an experiment for which $\left(\mathbf{N}_{n}, \mathbf{D}_{n}\right)$ is generated via Algorithm 4 but $K_{n}$ is chosen as the nodes of highest in-degree; results are shown in Figure 3b. The average error bound is slightly smaller for each $\kappa$ value than in Figure $3 \mathrm{a}$; this suggests that $K_{n}$ being the nodes of highest in-degree is indeed a good choice.

\section{DISCUSSION}

Before closing, we discuss several other aspects of our analysis, including the "optimal" choice of $K_{n}$, the restrictiveness of our assumptions, an example sequence $\left(\mathrm{N}_{n}, \mathbf{D}_{n}, \mathbf{U}_{n}\right)$ that satisfies our assumptions, a connection between our result and recent work on mixing times, and a geometric interpretation of our structural result. 

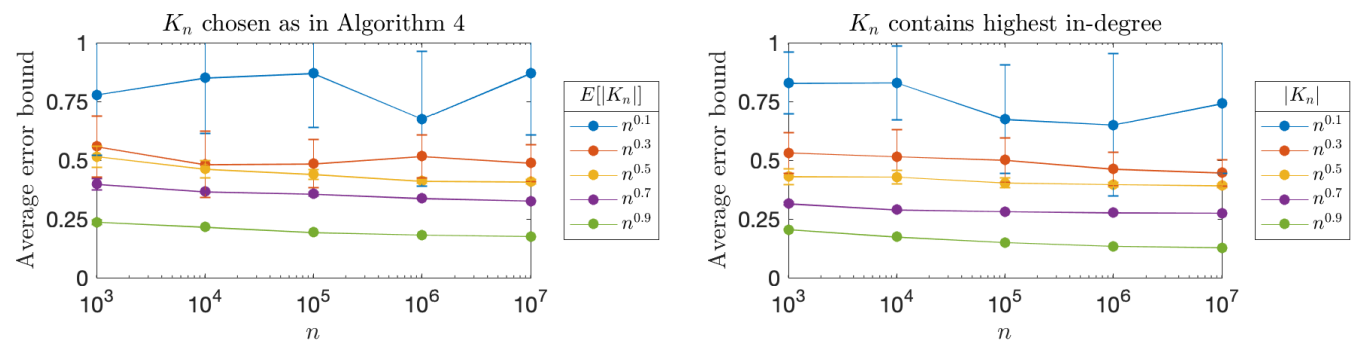

Fig. 3. For $s \sim V_{n} \backslash K_{n}$ uniformly on the DCM with the degrees from Algorithm 4, error decreases as $n$ grows.

\subsection{Choice of $K_{n}$}

A natural question is which choice of $K_{n}$ gives the smallest exponent in Theorem 1. For this, first note the exponent grows with $\kappa$ and $p$ and decays with $\delta(\epsilon, \tau, \rho$, and $\zeta$ also appear in the exponent, but all are independent of the choice of $K_{n}$ ). The growth with $\kappa$ and $p$ suggests a good choice of $K_{n}$ is a small set of nodes (small $\kappa$ ) containing a large fraction of instubs (small $p$ ). In particular, this suggests choosing $K_{n}$ to be the nodes with highest in-degree. We note the authors of [33] heuristically choose $K_{n}$ to be the nodes with highest global PageRank, and we showed in Section 5 that the complexity of a similar algorithm relates to $\Delta\left(K_{n}, \epsilon\right)$ on the DCM. Since global PageRank is suspected to correlate closely with in-degree for many graphs (see e.g. the aforementioned $[20,21,36,38,48,49]$ and the empirical works [2, 25]), our analysis appears to validate this heuristic. Unfortunately, we cannot yet prove that choosing the highest in-degree nodes as $K_{n}$ gives the smallest exponent; this is in part because exponent decays with $\delta$, which interacts with the choice of $K_{n}$ more subtly (see Assumption 1).

Choosing $K_{n}$ as nodes of high in-degree can also be motivated using recent results concerning the simple random walk on the DCM. In particular, it is known that the distribution of this walk is close to the stationary distribution after $\Theta(\log n)$ steps when starting from an arbitrary node (Theorem 1 in [17]), but is close to stationarity after just a constant number of steps when starting from the in-degree distribution (Theorem 3 in [17]). ${ }^{5}$ In other words, the in-degree distribution is an excellent initial guess for the stationary distribution. This further suggests that high in-degree nodes are typically reached quickly on random walks. On the other hand, our analysis states that $\pi_{v}$ is close to a linear combination of $\left\{\pi_{k}\right\}_{k \in K_{n}}$ when walks from $v$ are likely to hit $K_{n}$ (see Appendix A.1). In summary, choosing high in-degree nodes as $K_{n}$ means random walks are likely to reach $K_{n}$, which in turn means $\pi_{v}$ is likely to be well-approximated by $\left\{\pi_{k}\right\}_{k \in K_{n}}$.

\subsection{Comments on assumptions}

At a high level, our assumptions fall into two groups: the events $\left\{\Omega_{n, i}\right\}_{i=1}^{5}$ in Assumption 1, which say that the degree sequence is sparse, and the event $\Omega_{n, 6}$ in Assumption 1, which (in light of Assumption 2) says that a vanishing fraction of nodes contains a non-vanishing fraction of indegrees. We discuss each of these in turn, and then comment on their relative importance.

For the sparsity assumptions, we first note $\left\{\Omega_{n, i}\right\}_{i=1}^{3}$ in Assumption 1 are fairly standard given our approach, which leverages the fact that the random graph is locally tree-like [15]. In fact, $\Omega_{n, 3}$ is a weaker assumption than that required in, for example, [21]; see Appendix A.3 for details. Next,

\footnotetext{
${ }^{5}$ These results require $\max \left\{\max _{v \in V_{n}} D_{v}, \max _{v \in V_{n}} N_{v}\right\}=O(1)$, which is a stronger sparsity condition than we have assumed in this work. In fact, we suspect that max in-degree is not $O(1)$ for many sequences to which our results apply (see Sections 7.2 and 7.3), so this discussion is not entirely rigorous. Nevertheless, we believe it offers valuable insight.
} 


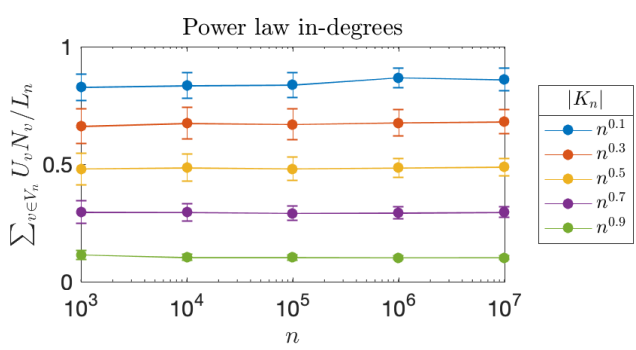

(a) $K_{n}$ is sublinear and contains a constant fraction of instubs. (Here $K_{n}$ are nodes of highest in-degree.)
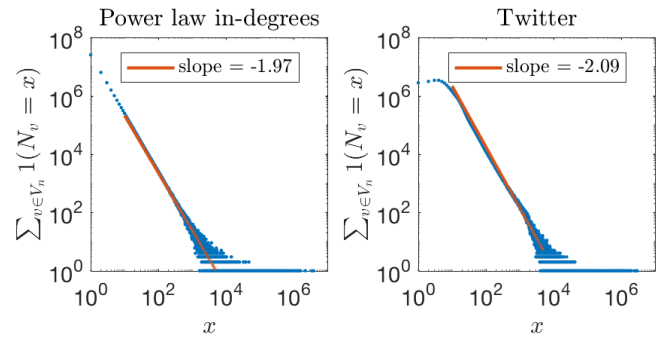

(b) The in-degrees for Fig. 4a are similar to in-degrees for the Twitter graph from [10]. (Here $n \approx 4 \times 10^{7}$.)

Fig. 4. $\Omega_{n, 6}$ is empirically satisfied with $\left|K_{n}\right|$ sublinear for power law in-degree distributions similar to Twitter.

we argue that the events $\Omega_{n, 4}, \Omega_{n, 5}$ in Assumption 1 are not restrictive. For this, first note that given $\Omega_{n, 1}$ and $\Omega_{n, 6}$ in Assumption 1, and since $\sum_{h=1}^{n} U_{h}=\Theta(n)$ by Assumption 2, we have

$$
\lim _{n \rightarrow \infty} \frac{\sum_{h=1}^{n} U_{h} N_{h}}{\sum_{h=1}^{n} U_{h}}=\lim _{n \rightarrow \infty} \frac{\frac{\sum_{h=1}^{n} U_{h} N_{h}}{\sum_{h=1}^{n} N_{h}} \frac{\sum_{h=1}^{n} N_{h}}{n}}{\frac{1}{n} \sum_{h=1}^{n} U_{h}}=p \eta_{1}<\infty,
$$

i.e. $\sum_{h=1}^{n} U_{h} N_{h} / \sum_{h=1}^{n} U_{h}$ converging to a finite limit is implied by other assumptions; additionally, Assumption 2 implicitly requires $\lambda^{*}=p \eta_{1}$. (We have written $\Omega_{n, 5}$ as its own assumption only out of convenience.) Similarly, $\Omega_{n, 4}$ is essentially implied by $\sum_{h=1}^{n} U_{h}=\Theta(n)$ and $\Omega_{n, 1}$, since then the fraction in $\Omega_{n, 4}$ satisfies

$$
\frac{\sum_{h=1}^{n} U_{h} D_{h}}{\sum_{h=1}^{n} U_{h}} \leq \frac{\frac{1}{n} \sum_{h=1}^{n} D_{h}}{\frac{1}{n} \sum_{h=1}^{n} U_{h}} \underset{n \rightarrow \infty}{\longrightarrow} \eta_{1}<\infty .
$$

For the remaining assumption, we recall that $\Omega_{n, 6}$ requires $\sum_{v \in V_{n}} U_{v} N_{v} / L_{n}$ to converge to $p<1$ with $\left|K_{n}\right|$ sublinear by Assumption 2. We offer empirical evidence that this occurs for certain graphs of interest. Specifically, in Figure $4 \mathrm{a}, \sum_{v \in V_{n}} U_{v} N_{v} / L_{n}$ remains constant and strictly less than 1 as $n$ grows, for a variety of sublinear $\left|K_{n}\right|$ choices (here we take $K_{n}$ to be the nodes of highest in-degree, as in Section 6). For this plot, in-degrees were sampled from a power law with exponent 2, i.e. $\mathbb{P}\left[N_{v}=i\right] \propto i^{-2}$. This in-degree distribution is commonly seen in real graphs and has been studied extensively, see e.g. [8,23]. As an example, Figure $4 \mathrm{~b}$ compares the histogram of these in-degrees with the in-degrees of the Twitter graph (available at [10] from WebGraph [13]). Both histograms are linear with slopes $\approx-2$ over $x \in[10,5000]$. In short, a common model of in-degree distributions empirically satisfies $\Omega_{n, 6}$ with $\left|K_{n}\right|$ sublinear.

Ultimately, we believe this last assumption is fundamentally necessary, while the sparsity assumptions may be artifacts of our analysis. To illustrate this, we compare the same power law in-degree sequence to a sequence of binomial in-degrees with parameters $n$ and $10 / n$ (i.e. Poisson(10) indegrees asymptotically). For both sequences, we first realize in-degrees independently and choose $K_{n}$ to be the nodes of highest in-degree; we then generate out-degrees as in Section 7.3. In Figure 5 a, we observe the moments appearing in $\left\{\Omega_{n, i}\right\}_{i=1}^{5}$ grow without bound as $n$ grows for the power law case but converge to constants for the binomial case. On the other hand, Figure 5b shows the quantity appearing in $\Omega_{n, 6}$ converges to $p<1$ for the power law case but rapidly approaches 1 for the binomial case. In short, the sparsity assumptions fail while the remaining assumption holds for power law in-degrees; the opposite is true for binomial in-degrees. From Figure 5c, we observe the average error bound (15) (computed as in Section 6) decays to 0 for the power law case but 


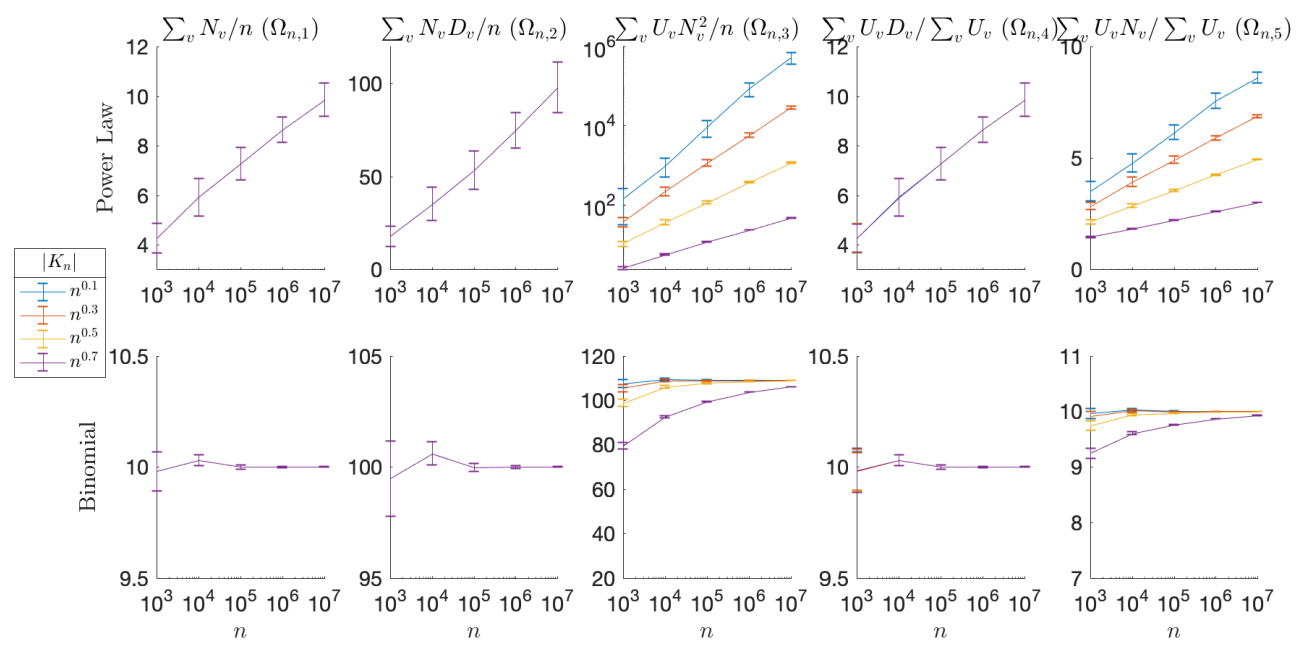

(a) Assumption 1 requires the quantities in $\left\{\Omega_{n, i}\right\}_{i=1}^{5}$ to converge to finite limits; this fails for the power law case (top row) but occurs for the binomial case (bottom row)
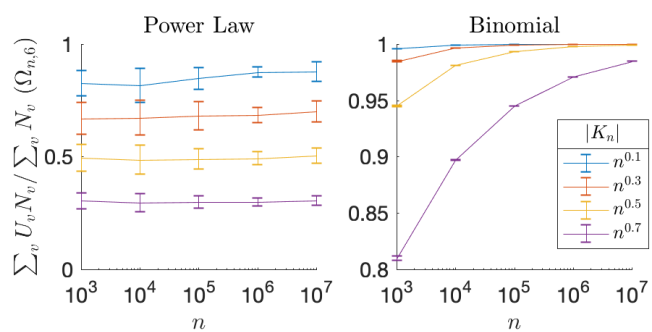

(b) Assumption 1 requires the quantity in $\Omega_{n, 6}$ to converge to $p<1$; this holds for the power law case (left) but fails for the binomial case (right)
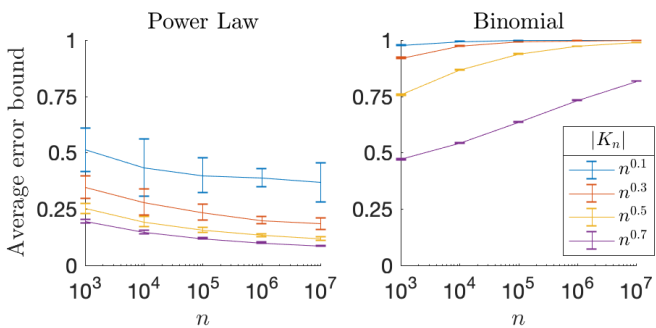

(c) The average error bound (15) decays to 0 for the power law case (left) but increases to 1 for the binomial case (right)

Fig. 5. Power law in-degrees satisfy only our most crucial assumption (Fig. 5a,5b), but average estimation error decreases, suggesting low dimensionality (Fig. 5c); the opposite is true for binomial in-degrees

grows to 1 for the binomial case. Hence, we ultimately conclude the following: when the sparsity assumptions fail but the remaining assumption holds, $\pi_{v}, v \notin K_{n}$ is typically well-approximated as a linear combination of $\left\{\pi_{k}\right\}_{k \in K_{n}}$, so our dimensionality measure should be small; when sparsity holds but the remaining assumption fails, the opposite is true. This suggests that the sparsity assumption is a less necessary condition for small dimensionality than the remaining assumption.

\subsection{Example degree sequence}

We next provide an example of a degree sequence satisfying Assumption 2. This is meant as a coarse model of a network like Twitter: roughly speaking, it will contain a small number of nodes with huge in-degrees (corresponding to celebrities on Twitter with millions of followers) and a large number of nodes with small in-degree (corresponding to "normal" users with tens or hundreds of followers); additionally, all nodes will have out-degrees that (in expectation) do not scale with $n$ (a given Twitter user does not follow a sizeable portion of all users). Specifically, given $c_{1}, c_{2}>0$ and 
$\kappa, l_{1}, l_{2} \in(0,1)$, we assign degrees and choose $K_{n}$ via Algorithm 4 . In words, we assign in-degrees as a mixture of two (truncated) power laws; after realizing in-degrees, each node initially receives one outstub, and the remaining $\sum_{w \in V_{n}}\left(N_{w}-1\right)$ outstubs are each assigned uniformly. (Note that this guarantees $N_{v}, D_{v} \in \mathbb{N} \forall v \in V_{n}$ and $\sum_{v \in V_{n}} N_{v}=\sum_{v \in V_{n}} D_{v}$, as we have assumed throughout the paper.) Proposition 2 states that the resulting sequence satisfies our assumptions.

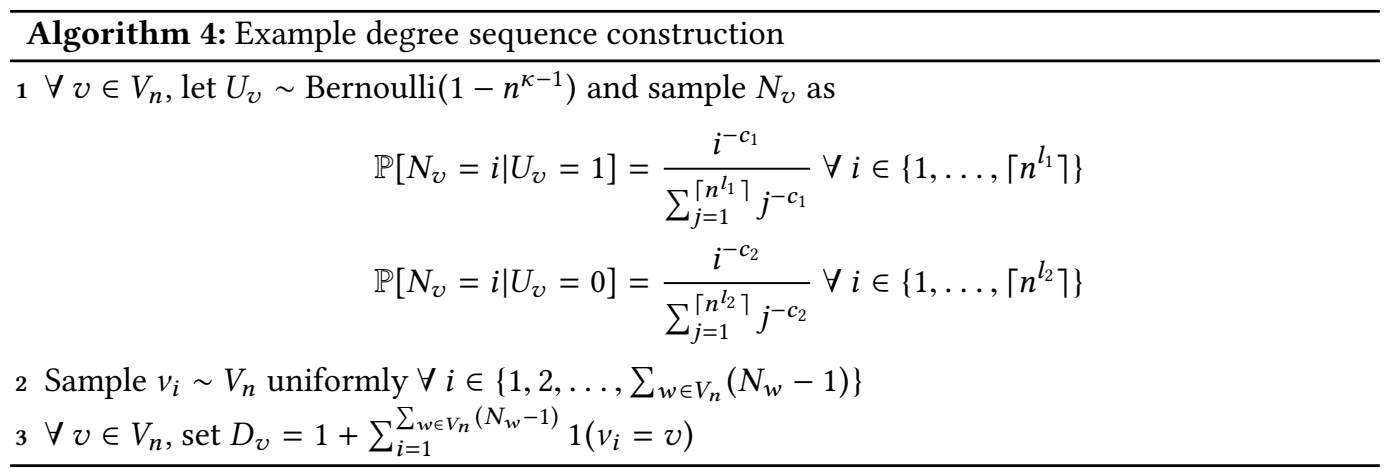

Proposition 2. Assume $c_{1} \in(3,4), c_{2} \in(1,2), l_{1} \in\left(0,1 /\left(5-c_{1}\right)\right), l_{2} \in(0,1)$, and $\kappa=1-l_{2}\left(2-c_{2}\right)$. Then the sequence $\left(\mathrm{N}_{n}, \mathbf{D}_{n}, \mathbf{U}_{n}\right)$ generated by Algorithm 4 satisfies Assumption 2.

Proof. See Appendix I.

As an example of parameter choices for Proposition 2, we can take $c_{1}=3.1, c_{2}=1.1, l_{1}=0.5$, $l_{2}=0.9$, and $\kappa=0.19$. In this case, nodes belonging to $V_{n} \backslash K_{n}$ have maximum in-degree $n^{0.5}$, while nodes in $K_{n}$ have maximum in-degree $n^{0.9}$; additionally, the in-degree distribution for $K_{n}$ has a heavier tail (since $c_{2}<c_{1}$ ). This is consistent with the discussion of Section 7.1, where we argued $K_{n}$ should contain high in-degree nodes.

We note that, per Figure 4, a more appropriate model for the Twitter in-degree sequence would be a power law with exponent $\approx 2$. However, Assumption 2 requires the second moment of $N_{v}$ to converge to a finite limit for $v \in V_{n} \backslash K_{n}$; when $N_{v}$ follows a power law for such $v$, this requires the exponent to exceed 3 (hence the requirement $c_{1}>3$ in Proposition 2). On the other hand, recall that our analysis uses a result from [21] but with weaker assumptions. Specifically, we only require $\sum_{v} U_{v} N_{v}^{2}=O(n)$, whereas [21] requires $\sum_{v} N_{v}^{2}=O(n)$ (see Appendix A.3). Weakening the assumption in this manner allows for $N_{v}, v \in K_{n}$ to follow a power law with exponent $c_{2} \in(1,2)$ in Proposition 2. This is turn yields an in-degree distribution with a heavier tail than if all in-degrees were restricted to power law with exponent exceeding 3 , which allows our model to more closely resemble the exponent $\approx 2$ case. In particular, $c_{1} \in(3,4), c_{2} \in(1,2)$ yields an in-degree sequence with bounded mean but unbounded variance as $n \rightarrow \infty$, as does a power law with e.g. exponent 2.1.

Finally, we note $c_{1}<4$ is not necessary to prove that Assumption 2 holds. We only require $c_{1}<4$ to avoid addressing separate cases in the proof; additionally, taking $c_{1}>4$ would yield a less accurate model of power law sequences observed in practice, which often have exponents $\approx 2$.

\subsection{Connection to mixing times}

We can also motivate our choice of $\alpha_{n}$ in terms of the mixing time of the simple random walk on $G_{n}$. First, we let $\pi$ denote the stationary distribution of this walk. For any $v \in \mathbb{N}$, and for a graph of $n$ nodes, we let $\pi_{v}$ be the uniform distribution on $V_{n}$ for $n<v$, and we define $\pi_{v}$ as in Definition 1 for $n \geq v$. (This ensures $\pi_{v}$ is well-defined in what follows.) We can then prove the following, 
which shows that $\pi_{v}$ is (asymptotically) indistinguishable from $\pi$ when $\alpha_{n}=o(1 / \log n)$ and when a certain mixing condition is satisfied.

Proposition 3. Let $v \in \mathbb{N}, m=\Theta(\log n), \alpha_{n}=o(1 / \log n)$, and $\epsilon>0$. Then for $n$ sufficiently large, we have for any $G_{n}$,

$$
\left\|\pi_{v}-\pi\right\|_{1} \leq 3 \max _{w \in V_{n}}\left\|e_{w}^{\top} P^{m}-\pi\right\|_{1}+\epsilon
$$

As a consequence, if

$$
\max _{w \in V_{n}}\left\|e_{w}^{\top} P^{m}-\pi\right\|_{1} \underset{n \rightarrow \infty}{\stackrel{P}{\longrightarrow}} 0
$$

then $\left\|\pi_{v}-\pi\right\|_{1} \underset{n \rightarrow \infty}{\stackrel{\mathbb{P}}{\longrightarrow}} 0$, where $\stackrel{\mathbb{P}}{\rightarrow}$ denotes convergence in probability.

Proof. See Appendix H.

Proposition 3 states that when $\alpha_{n} \log n \rightarrow 0$ and the random walk on $G_{n}$ mixes in $\log n$ steps (in the sense of (16)), $\pi_{v}$ is close to $\pi$ in the $l_{1}$ norm (for large $n$ and with high probability). Put differently, the matrix with rows $\left\{\pi_{v}\right\}_{v \in V_{n}}$ is close to the rank one matrix $1_{n} \pi$ in this case, suggesting a dimensionality of 1 . This is in fact somewhat obvious: since the first jump to $v$ on the Markov chain defining $\pi_{v}$ occurs at time $1 / \alpha_{n}$ (in expectation), the chain reaches stationarity after $\log n$ steps but does not jump to $v$ until (for example) $(\log n)^{2}$ steps, so $\pi_{v}$ should not depend on $v$.

We believe that the mixing condition (16) holds for our graph model. This belief is based on recent work by Bordenave, Caputo, and Salez, who prove (16) for a class of sparse, randomly-generated Markov chains (Theorem 1 in [16]). In particular, this class includes random walks on random graphs with a given degree sequence (i.e. the DCM). The key differences between this model and ours are (1) we permit multi-edges, while the model in [16] does not, and (2) $D_{v}>1, D_{v}=O(1) \forall v \in V_{n}$ in the [16] model. ${ }^{6}$ We note that the condition $D_{v}>1, D_{v}=O(1)$ can be added to Assumption 1 without contradiction; Assumption 2 then implies a graph with a few huge in-degrees, mostly small in-degrees, and all small out-degrees, as in Section 7.3.

We have thus far argued the dimensionality of $\left\{\pi_{v}\right\}_{v \in V_{n}}$ grows as $n^{c}$ for some $c \in(0,1)$ when $\alpha_{n}=\Theta(1 / \log n)\left(\right.$ Theorem 1) and is constant when $\alpha_{n}=o(1 / \log n)$ (Proposition 3). A third case is $\alpha_{n}=\omega(1 / \log n)$. For this case, we first note $[16,17]$ prove a matching lower bound to (16), i.e. they show for some $m^{\prime}=\Theta(\log n)$,

$$
\min _{w \in V_{n}}\left\|e_{w}^{\top} P^{m^{\prime}}-\pi\right\|_{T V} \underset{n \rightarrow \infty}{\stackrel{\mathbb{P}}{\longrightarrow}} 1
$$

where $\|\cdot\|_{T V}$ denotes total variation distance. Hence, the number of jumps to $v$ before mixing scales with $\alpha_{n} \log n$ in expectation, which is unbounded in the case $\alpha_{n}=\omega(1 / \log n)$. In contrast, only a constant number of jumps to $v$ occur before mixing in the $\alpha_{n}=\Theta(1 / \log n)$ case. For this reason, we suspect, but currently lack the lower bound to prove, that $\Delta\left(K_{n}, \epsilon\right)$ behaves fundamentally differently if $\alpha_{n}=\omega(1 / \log n)$, perhaps dominating $n^{c}$ for any $c \in(0,1)$ (e.g., $n / \log n$ or even $n$ ).

Ultimately, this discussion further explains our choice of $\alpha_{n}$ : if we set $\alpha_{n}$ much smaller, we obtain dimensionality 1 ; if we set $\alpha_{n}$ much larger, we expect to obtain a much larger dimensionality. Hence, our choice of $\alpha_{n}$ yields the strongest possible result before trivial behavior occurs.

\footnotetext{
${ }^{6}$ This is actually slightly stronger than the condition in [16]; see Example 1 in [16] for details. We have stated the stronger version for simplicity.
} 


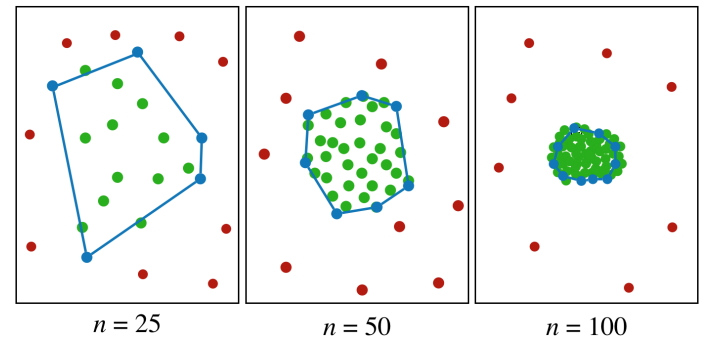

Fig. 6. As $n$ grows, most of $\left\{\pi_{v}\right\}_{v \in V_{n} \backslash K_{n}}$ (green dots) concentrate near the convex hull of $\left\{\pi_{k}\right\}_{k \in K_{n}}$ (blue dots/lines) (a few of $\left\{\pi_{v}\right\}_{v \in V_{n} \backslash K_{n}}$ (red dots) can be far away). Further, since $\left|K_{n}\right|$ shrinks relative to $n$, the convex hull of $\left\{\pi_{k}\right\}_{k \in K_{n}}$ shrinks relative to the $n$-dimensional simplex.

\subsection{Geometric interpretation}

Finally, we note Theorem 1 has a simple geometric interpretation. To see this, first recall that for all but a vanishing fraction of $v \in V_{n} \backslash K_{n}$, the theorem states

$$
\left\|\pi_{v}-\frac{\sum_{k \in K_{n}} \tilde{\pi}_{v}(k) \pi_{k}}{\alpha_{n}+\left(1-\alpha_{n}\right) \tilde{\pi}_{v}\left(K_{n}\right)}\right\|<\epsilon .
$$

Furthermore, for such $v$, we have by (106) in Appendix G,

$$
\frac{\tilde{\pi}_{v}\left(K_{n}\right)}{\alpha_{n}+\left(1-\alpha_{n}\right) \tilde{\pi}_{v}\left(K_{n}\right)} \geq \frac{1}{\frac{\epsilon+\alpha_{n}\left(2-\left(\epsilon+\alpha_{n}\right)\right)}{1-\left(\epsilon+\alpha_{n}\right)}+\left(1-\alpha_{n}\right)} .
$$

Note the left side of (17) is upper bounded by 1 , while the right side tends to $1-\epsilon$ as $n \rightarrow \infty$. The previous two equations can then be interpreted as follows: setting $\epsilon$ arbitrarily small, and letting $n$ grow large, $\pi_{v}$ is arbitrarily close to a linear combination $\left\{\pi_{k}\right\}_{k \in K_{n}}$; furthermore, the weights for the linear combination are nonnegative and their sum is arbitrarily close to 1 . Taken together, $\pi_{v}$ is arbitrarily close to the convex hull of $\left\{\pi_{k}\right\}_{k \in K_{n}}$. Additionally, because $B_{v}\left(K_{n}, \epsilon\right)$ fails with high probability, all but a vanishing fraction of $\left\{\pi_{v}\right\}_{v \in V_{n}}$ are arbitrarily close to this convex hull. Finally, because $\left|K_{n}\right|$ scales sublinearly in $n$, this convex hull is a low dimensional subset of the $n$-dimensional simplex to which $\left\{\pi_{v}\right\}_{v \in V_{n}}$ belong. Hence, beyond describing the dimensionality of the set of PPR vectors, our main result also describes the space in which most of these vectors reside. This interpretation is depicted graphically in Figure 6; we note this figure is simply an illustration of the preceding paragraph and was not generated using actual PPR vectors. ${ }^{7}$

\section{CONCLUSIONS}

In this work, we argued (analytically for the DCM and empirically for other graphs) that the dimensionality of the set of PPR vectors $\left\{\pi_{v}\right\}_{v \in V_{n}}$ scales sublinearly in $n$. We also used our analysis to bound the complexity of an algorithm to compute all PPR vectors, which is similar to that found in [33]. Our analysis suggests several avenues for future work. First, the proof of Lemma 1 can be modified to analyze the tail of the $l_{\infty}$ error (this would essentially involve replacing Lemma 6 with a tail bound on a maximum instead of a sum). Hence, bounding absolute error for the estimate of $\pi_{s}(v)$ for any $v \in V_{n}$ is a straightforward extension; a less immediate analysis would involve bounding relative error. Second, examining PPR dimensionality for other random graph models

\footnotetext{
${ }^{7}$ Generating such a figure with actual PPR vectors is difficult because $n$-dimensional vectors must be projected into $2 \mathrm{D}$ space while approximately preserving $l_{1}$ distances, and such a projection is not well understood (see e.g. [19, 32]). Appendix B.4 includes a figure obtained from actual PPR vectors, but it is less illustrative than Figure 6 .
} 
may be of interest. For example, several papers have analyzed PPR on preferential attachment models [6,27]; we suspect a dimensionality analysis for such graphs would yield a message similar to our work ( $K_{n}$ should contain nodes with high in-degrees). A more interesting class of graphs would be from the stochastic block model; here it may be more beneficial to choose $K_{n}$ such that each community contains a nonempty subset of $K_{n}$. Finally, as discussed in Section 5.2, we believe our analysis and our approach to analyzing the algorithm from [33] can be used to design improved versions of existing algorithms and to derive tighter complexity bounds.

\section{REFERENCES}

[1] David J Aldous, Antar Bandyopadhyay, et al. 2005. A survey of max-type recursive distributional equations. The Annals of Applied Probability 15, 2 (2005), 1047-1110.

[2] Brian Amento, Loren Terveen, and Will Hill. 2000. Does "authority" mean quality? Predicting expert quality ratings of Web documents. In Proceedings of the 23rd annual international ACM SIGIR conference on Research and development in information retrieval. ACM, 296-303.

[3] Reid Andersen, Christian Borgs, Jennifer Chayes, John Hopcroft, Vahab Mirrokni, and Shang-Hua Teng. 2008. Local computation of PageRank contributions. Internet Mathematics 5, 1-2 (2008), 23-45.

[4] Reid Andersen, Fan Chung, and Kevin Lang. 2006. Local graph partitioning using PageRank vectors. In 2006 IEEE Symposium on Foundations of Computer Science. IEEE, 475-486.

[5] Konstantin Avrachenkov, Arun Kadavankandy, Liudmila Ostroumova Prokhorenkova, and Andrei Raigorodskii. 2015. PageRank in undirected random graphs. In International Workshop on Algorithms and Models for the Web-Graph. Springer, 151-163.

[6] Konstantin Avrachenkov and Dmitri Lebedev. 2006. PageRank of scale-free growing networks. Internet Mathematics 3 , 2 (2006), 207-231.

[7] Konstantin Avrachenkov, Nelly Litvak, Danil Nemirovsky, and Natalia Osipova. 2007. Monte Carlo methods in PageRank computation: When one iteration is sufficient. SIAM J. Numer. Anal. 45, 2 (2007), 890-904.

[8] Albert-László Barabási and Réka Albert. 1999. Emergence of scaling in random networks. Science 286, 5439 (1999), 509-512.

[9] Edward A Bender and E Rodney Canfield. 1978. The asymptotic number of labeled graphs with given degree sequences. Journal of Combinatorial Theory, Series A 24, 3 (1978), 296-307.

[10] Paolo Boldi, Massimo Santini, and Sebastiano Vigna. [n. d.]. Laboratory for Web Algorithmics datasets. http: //law.di.unimi.it/datasets.php.

[11] Paolo Boldi, Massimo Santini, and Sebastiano Vigna. 2005. PageRank as a function of the damping factor. In Proceedings of the 14th international conference on World Wide Web. ACM, 557-566.

[12] Paolo Boldi, Massimo Santini, and Sebastiano Vigna. 2009. PageRank: functional dependencies. ACM Transactions on Information Systems (TOIS) 27, 4 (2009), 19.

[13] Paolo Boldi and Sebastiano Vigna. 2004. The WebGraph framework I: Compression techniques. In Proceedings of the 13th International Conference on World Wide Web. ACM, 595-602.

[14] Béla Bollobás. 1980. A probabilistic proof of an asymptotic formula for the number of labelled regular graphs. European Journal of Combinatorics 1, 4 (1980), 311-316.

[15] Charles Bordenave. [n. d.]. Lecture notes on random graphs and probabilistic combinatorial optimization. https: //www.math.univ-toulouse.fr/ bordenave/coursRG.pdf.

[16] Charles Bordenave, Pietro Caputo, and Justin Salez. 2016. Cutoff at the "entropic time" for sparse Markov chains. Probability Theory and Related Fields (2016), 1-32.

[17] Charles Bordenave, Pietro Caputo, and Justin Salez. 2018. Random walk on sparse random digraphs. Probability Theory and Related Fields 170, 3-4 (2018), 933-960.

[18] Christian Borgs, Michael Brautbar, Jennifer Chayes, and Shang-Hua Teng. 2014. Multiscale matrix sampling and sublinear-time pagerank computation. Internet Mathematics 10, 1-2 (2014), 20-48.

[19] Moses Charikar and Amit Sahai. 2002. Dimension reduction in the $l_{1}$ norm. In 2002 IEEE Symposium on Foundations of Computer Science. IEEE, 551-560.

[20] Ningyuan Chen, Nelly Litvak, and Mariana Olvera-Cravioto. 2014. PageRank in scale-free random graphs. In International Workshop on Algorithms and Models for the Web-Graph. Springer, 120-131.

[21] Ningyuan Chen, Nelly Litvak, and Mariana Olvera-Cravioto. 2017. Generalized PageRank on directed configuration networks. Random Structures \& Algorithms 51, 2 (2017), 237-274.

[22] Ningyuan Chen and Mariana Olvera-Cravioto. 2013. Directed random graphs with given degree distributions. Stochastic Systems 3, 1 (2013), 147-186. 
[23] Aaron Clauset, Cosma Rohilla Shalizi, and Mark EJ Newman. 2009. Power-law distributions in empirical data. SIAM Rev. 51, 4 (2009), 661-703.

[24] D.P. Dubhashi and A. Panconesi. 2009. Concentration of Measure for the Analysis of Randomized Algorithms. Cambridge University Press. https://books.google.com/books?id=UUohAwAAQBAJ

[25] Santo Fortunato, Marián Boguñá, Alessandro Flammini, and Filippo Menczer. 2006. Approximating PageRank from in-degree. In International Workshop on Algorithms and Models for the Web-Graph. Springer, 59-71.

[26] R.G. Gallager. 2013. Stochastic Processes: Theory for Applications. Cambridge University Press. https://books.google. com/books?id=ERLrAQAAQBAJ

[27] Alessandro Garavaglia, Remco van der Hofstad, and Nelly Litvak. 2018. Local weak convergence for PageRank. arXiv preprint arXiv:1803.06146 (2018).

[28] David F Gleich. 2015. PageRank beyond the Web. SIAM Rev. 57, 3 (2015), 321-363.

[29] Pankaj Gupta, Ashish Goel, Jimmy Lin, Aneesh Sharma, Dong Wang, and Reza Zadeh. 2013. WTF: The who to follow service at Twitter. In Proceedings of the 22nd international conference on World Wide Web. ACM, 505-514.

[30] Will Hamilton, Zhitao Ying, and Jure Leskovec. 2017. Inductive representation learning on large graphs. In Advances in Neural Information Processing Systems. 1024-1034.

[31] Taher H Haveliwala. 2002. Topic-sensitive PageRank. In Proceedings of the 11th International Conference on World Wide Web. ACM, 517-526.

[32] Piotr Indyk. 2000. Stable distributions, pseudorandom generators, embeddings and data stream computation. In 2000 IEEE Symposium on Foundations of Computer Science. IEEE, 189-197.

[33] Glen Jeh and Jennifer Widom. 2003. Scaling personalized web search. In Proceedings of the 12th International Conference on World Wide Web. ACM, 271-279.

[34] A.J. Laub. 2005. Matrix Analysis for Scientists and Engineers. Society for Industrial and Applied Mathematics (SIAM, 3600 Market Street, Floor 6, Philadelphia, PA 19104). https://books.google.com/books?id=nCsLwYJdOlwC

[35] Daniel D Lee and H Sebastian Seung. 2001. Algorithms for non-negative matrix factorization. In Advances in neural information processing systems. 556-562.

[36] Jiung Lee and Mariana Olvera-Cravioto. 2017. PageRank on inhomogeneous random digraphs. arXiv preprint arXiv:1707.02492 (2017).

[37] Jure Leskovec and Andrej Krevl. [n. d.]. SNAP Datasets: Stanford Large Network Dataset Collection. http://snap. stanford.edu/data.

[38] Nelly Litvak, Werner RW Scheinhardt, and Yana Volkovich. 2007. In-degree and PageRank: Why do they follow similar power laws? Internet Mathematics 4, 2-3 (2007), 175-198.

[39] Peter Lofgren, Siddhartha Banerjee, and Ashish Goel. 2016. Personalized PageRank estimation and search: A bidirectional approach. In Proceedings of the Ninth ACM International Conference on Web Search and Data Mining. ACM, 163-172.

[40] Peter Lofgren and Ashish Goel. 2013. Personalized pagerank to a target node. arXiv preprint arXiv:1304.4658 (2013).

[41] Ivan Markovsky. 2011. Low rank approximation: algorithms, implementation, applications. Springer Science \& Business Media.

[42] Michael Molloy and Bruce Reed. 1995. A critical point for random graphs with a given degree sequence. Random structures \& algorithms 6, 2-3 (1995), 161-180.

[43] Lawrence Page, Sergey Brin, Rajeev Motwani, and Terry Winograd. 1999. The PageRank citation ranking: Bringing order to the web. Technical Report. Stanford InfoLab.

[44] Rajat Sen, Karthikeyan Shanmugam, Murat Kocaoglu, Alexandros G Dimakis, and Sanjay Shakkottai. 2016. Contextual bandits with latent confounders: An NMF approach. arXiv preprint arXiv:1606.00119 (2016).

[45] Kijung Shin, Jinhong Jung, Sael Lee, and U Kang. 2015. Bear: Block elimination approach for random walk with restart on large graphs. In Proceedings of the 2015 ACM SIGMOD International Conference on Management of Data. ACM, 1571-1585.

[46] Hanghang Tong, Christos Faloutsos, and Jia-Yu Pan. 2008. Random walk with restart: Fast solutions and applications. Knowledge and Information Systems 14, 3 (2008), 327-346.

[47] Remco van der Hofstad, Gerard Hooghiemstra, and Piet Van Mieghem. 2005. Distances in random graphs with finite variance degrees. Random Structures \& Algorithms 27, 1 (2005), 76-123.

[48] Yana Volkovich and Nelly Litvak. 2010. Asymptotic analysis for personalized web search. Advances in Applied Probability 42, 2 (2010), 577-604.

[49] Yana Volkovich, Nelly Litvak, and Debora Donato. 2007. Determining factors behind the PageRank log-log plot. In International Workshop on Algorithms and Models for the Web-Graph. Springer, 108-123.

[50] Nicholas C Wormald. 1980. Some problems in the enumeration of labelled graphs. Bulletin of the Australian Mathematical Society 21, 1 (1980), 159-160. 
[51] Zhanxing Zhu, Zhirong Yang, and Erkki Oja. 2013. Multiplicative updates for learning with stochastic matrices. In Scandinavian Conference on Image Analysis. Springer, 143-152. 
Note on the organization of appendices: Appendix A outlines the proof of Lemma 1, with details deferred to Appendix D. Appendix B contains details on the experiments from Section 6, and Appendix $\mathrm{C}$ contains details on the algorithmic comparisons from Section 5.2. Finally, Appendices E, F, G, H, and I contain the proofs of Proposition 1, Theorem 1, Theorem 2, Proposition 3, and Proposition 2, respectively.

\section{A LEMMA 1 PROOF OUTLINE}

In this appendix, we outline the proof of Lemma 1. Our approach follows the outline described in Section 4.3. Specifically, we consider Steps 1-4 of the outline in Appendices A.1-A.4, respectively. In Appendix A.5, we combine the results to prove the lemma.

\section{A.1 Error bound in $m$-step neighborhood}

Our first goal is to bound the error term

$$
\left\|\pi_{s}-\left(\alpha_{n} e_{s}^{\top}+\sum_{k \in K_{n}} \beta_{s}(k) \pi_{k}\right)\right\|_{1}
$$

for a particular choice of $\left\{\beta_{s}(k)\right\}_{k \in K_{n}}$. For this, we require an intermediate result; namely, (7) from Section 4.3, which we formalize as Lemma 2 here. Recall from Section 4.3 that $\tilde{\pi}_{s}$ is the stationary distribution of the Markov chain with transition matrix $\tilde{P}_{s}=\left(1-\alpha_{n}\right) \tilde{P}+\left(\alpha_{n} e_{V_{n} \backslash K_{n}}+e_{K_{n}}\right) e_{s}^{\top}$, where $\tilde{P}$ satisfies $\tilde{P}(i, j)=U_{i} P(i, j)$.

As mentioned in Section 4, Lemma 2 is an alternate formulation of the Hubs Theorem from [33]. At a high level, both formulations view $\pi_{s}(v)$ as the probability of paths from $s$ to $v$ and partition these paths into those that avoid $K_{n}$ (which have probability proportional to $\tilde{\pi}_{s}(v)$ ) and those through $K_{n}$ (which have probability proportional to $\tilde{\pi}_{s}(k) \pi_{k}(v)$ ). The difference between the two formulations is that we explicitly construct a new Markov chain that does not include paths through $K_{n}$ (i.e. the chain with transition matrix $\tilde{P}_{s}$ ), while [33] does not. Our formulation admits an intuitive probabilistic proof; in contrast, the proof in [33] is linear algebraic.

LEMMA 2. If $U_{s}=1$, we have for any realization of the DCM,

$$
\pi_{s}(v)=\frac{\alpha_{n} U_{v} \tilde{\pi}_{s}(v)+\sum_{k \in K_{n}} \tilde{\pi}_{s}(k) \pi_{k}(v)}{\alpha_{n}+\left(1-\alpha_{n}\right) \tilde{\pi}_{s}\left(K_{n}\right)} \forall v \in V_{n} .
$$

Proof. See Appendix D.1.

We next bound the error term (18) using a particular $\left\{\beta_{s}(k)\right\}_{k \in K_{n}}$; namely, that suggested by Lemma 2. Our bound leverages the fact that the transition matrix $\tilde{P}_{s}$ is written as the sum of two matrices, one of which is rank one. This allows us to use the Sherman-Morrison-Woodbury formula (see e.g. Section 6.4 of [34]) to derive a bound on the error term in terms of the row vector

$$
\mu_{s}^{(m)}=e_{s}^{\mathrm{T}} \sum_{j=0}^{m}\left(1-\alpha_{n}\right)^{j} \tilde{P}^{j},
$$

which clearly depends only on the $m$ step neighborhood out of $s$.

Lemma 3. Consider any realization of the DCM and assume $U_{s}=1$. For all $k \in K_{n}$, define

$$
\beta_{s}(k)=\frac{\tilde{\pi}_{s}(k)}{\alpha_{n}+\left(1-\alpha_{n}\right) \tilde{\pi}_{s}\left(K_{n}\right)} .
$$


Then for each $m \in \mathbb{N}$,

$$
\left\|\pi_{s}-\left(\alpha_{n} e_{s}^{\top}+\sum_{k \in K_{n}} \beta_{s}(k) \pi_{k}\right)\right\|_{1} \leq \alpha_{n}\left(\mu_{s}^{(m-1)}\left(V_{n} \backslash K_{n}\right)-1\right)+e_{s}^{\top}\left(1-\alpha_{n}\right)^{m} \tilde{P}^{m} e_{V_{n} \backslash K_{n}} .
$$

Proof. See Appendix D.2.

\section{A.2 Coupling with branching process (Step 2)}

Next, we show that the error bound in Lemma 3 follows the same distribution as a related quantity defined in terms of a branching process. Before presenting this result, we formally define the DCM construction and the branching process.

We begin with the DCM. As described in Section 2.1, the basic idea is to randomly pair outgoing half-edges (which we call outstubs) with incoming half-edges (which we call instubs) in a breadthfirst search fashion. We begin by sampling a node $s$ uniformly at random from $V_{n}$. In the first iteration, for each outstub belonging to $s$, we sample an instub uniformly (resampling if the sampled instub has already been paired), and we pair the outstub and instub. We allow the possibility that the sampled instub belongs to $s$ (in which case a self-loop is formed) or that multiple outstubs of $s$ are paired with instubs belonging to the same node (in which case multiple edges are formed between $s$ and that node). ${ }^{8}$

At the conclusion of the first iteration, we denote by $A_{1}$ the subset of $V_{n} \backslash\{s\}$ containing those nodes that have had at least one instub paired with an outstub of $s$. In the second iteration, we pair all outstubs of all nodes in $A_{1}$ in the manner described previously. In general, we pair all outstubs of all nodes in $A_{m-1}$ during the $m$-th iteration, where $A_{m-1}$ is the set of nodes $v$ at distance $m-1$ from $s$. In other words, paths out of $s$ of length $m$ are constructed during the $m$-th iteration. When all outstubs of all nodes have been paired, the construction is complete.

To facilitate the graph construction and the coupling argument, we define labels for each instub $e$ and for each node $v$, denoted $g(e)$ and $g(v)$. The instub label $g(e)$ is necessary because if $e$ is sampled for pairing, we must check whether $e$ has already been paired. Hence, we define

$$
g(e)=\left\{\begin{array}{ll}
1, & e \text { is currently unpaired } \\
0, & e \text { is currently paired }
\end{array}\right. \text {. }
$$

The node label $g(v)$ is defined as

$$
g(v)=\left\{\begin{array}{ll}
A, & v \text { does not currently belong to graph } \\
B, & v \text { belongs to graph, } U_{v}=0 \\
C, & v \text { belongs to graph, } U_{v}=1, \text { all paths from } s \text { to } v \text { visit some } w \in V_{n} \text { s.t. } U_{w}=0 \\
D, & v \text { belongs to graph, } U_{v}=1, \text { some path from } s \text { to } v \text { avoids all } w \in V_{n} \text { s.t. } U_{w}=0
\end{array} .\right.
$$

To illustrate these node labels, we show a graph after three iterations of the construction in Figure 7. The node at the top of the figure is $s$. Circle and square nodes, respectively, depict those nodes $v$ with $U_{v}=1$ and $U_{v}=0$, respectively (i.e., those belonging to $V_{n} \backslash K_{n}$ and $K_{n}$, respectively). Short arrows depict half-edges (i.e. unpaired instubs and outstubs), while longer arrows depict

\footnotetext{
${ }^{8}$ Because of this, the resulting graph will in general be a multi-graph. We note the authors of [22] prove that a simple graph (no self-loops or multi-edges) results with positive probability as $n \rightarrow \infty$; however, this requires stronger assumptions on the degree sequence than Assumption 1, which is all that we require to prove our main result. Specifically, guaranteeing that a simple graph emerges with positive probability as $n \rightarrow \infty$ requires empirical variances of the in- and out-degree sequences to converge.
} 


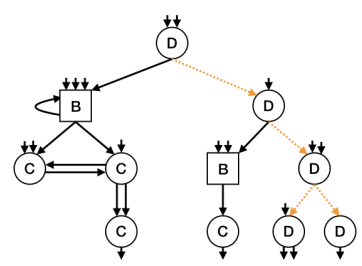

Fig. 7. Example graph after three iterations; $\mu_{s}^{(3)}\left(V_{n} \backslash K_{n}\right)$ depends only on orange dashed subgraph.

edges (i.e. instubs and outstubs that have been paired). Node labels, assigned according to (20), are displayed on each node.

Node labels will be useful in the coupling argument to come. In particular, the term $\mu_{s}^{(m)}\left(V_{n} \backslash K_{n}\right)$ in Lemma 3 only depends on the subgraph containing label $D$ nodes within $m$ steps of $s$ (depicted by orange dashed edges in Figure 7 for $m=3$ ). This observation follows since $\mu_{s}^{(m)}(v)$ (by definition) is nonzero if and only if there exists a path from $s$ to $v$ of length at most $m$ that avoids $K_{n}$.

The formal graph construction is given in Algorithm 5. Most notation has already been defined, but we use three additional pieces of notation in the algorithm: $I_{n}$ is the set of all instubs, $\left\{\left(v^{\prime}, j\right)\right\}_{j=1}^{D_{v^{\prime}}}$ is the set of outstubs belonging to $v^{\prime} \in V_{n}$ (which are ordered arbitrarily), and $\tau_{G}$ is a variable that tracks the first iteration at which certain events occur (these events relate to the coupling and will be discussed shortly).

Before proceeding, we offer several comments to relate Algorithm 5 to the preceding description of the construction:

- In Line 1-2, we initialize the algorithm. Namely, we sample the first node $s$, define the label $g(s)$ according to (20), and set $A_{0}=\{s\}$ (i.e. the only node at distance zero from $s$ is $s$ itself). We then set $g(e)=1$ for all instubs $e$ (since no instubs have been paired) and $g(v)=A \forall v \neq s$ (since only $s$ belongs to the graph at this stage of the algorithm).

- The remainder of the algorithm involves iterating over $m$ (outer for loop), iterating over nodes $v^{\prime}$ at distance $m-1$ from $s$ (middle for loop), and iterating over outstubs belonging to $v^{\prime}$ (inner for loop). For each such outstub, denoted $\left(v^{\prime}, j\right)$, the following steps occur:

- In Lines 8-12, we uniformly sample an instub $e$, resampling (if needed) until an unpaired instub is found. (Line 9 relates to the coupling and will be discussed shortly.)

- After sampling an unpaired instub $e$, we pair $\left(v^{\prime}, j\right)$ with $e$ and set $g(e)=0$ to reflect the fact that $e$ has been paired (Line 13). If the node $v$ to which $e$ belongs did not previously belong to the graph (i.e. if $g(v)=A$ ), then $v$ is at distance $m$ from $s$, so we add $v$ to $A_{m}$ (Line 14). (Line 15 relates to the coupling and will be discussed shortly.)

- In Lines 17-20, we update the label of $v$ according to (20). Note that, if $g\left(v^{\prime}\right)=D$ and $g(v)=C,(20)$ implies that a path from $s$ to $v$ avoiding $K_{n}$ did not exist before $\left(v^{\prime}, j\right)$ and $e$ were paired, but now such a path does exist. Hence, if some node $w$ s.t. $g(w)=C$ can be reached from $v$ while avoiding $K_{n}$, a path from $s$ to $w$ avoiding $K_{n}$ now exists as well. For this reason, we must change the label of such $w$ from $C$ to $D$ (Line 20).

- After these steps occur, if all instubs have been paired, the algorithm terminates (Line 22).

Our next goal is to define a branching process and a quantity related to the error bound in Lemma 3, so that this error bound can instead be analyzed on the tree resulting from the branching process. Before defining this tree construction, we offer some intuition, which also helps explain the variable $\tau_{G}$ in Algorithm 5. 


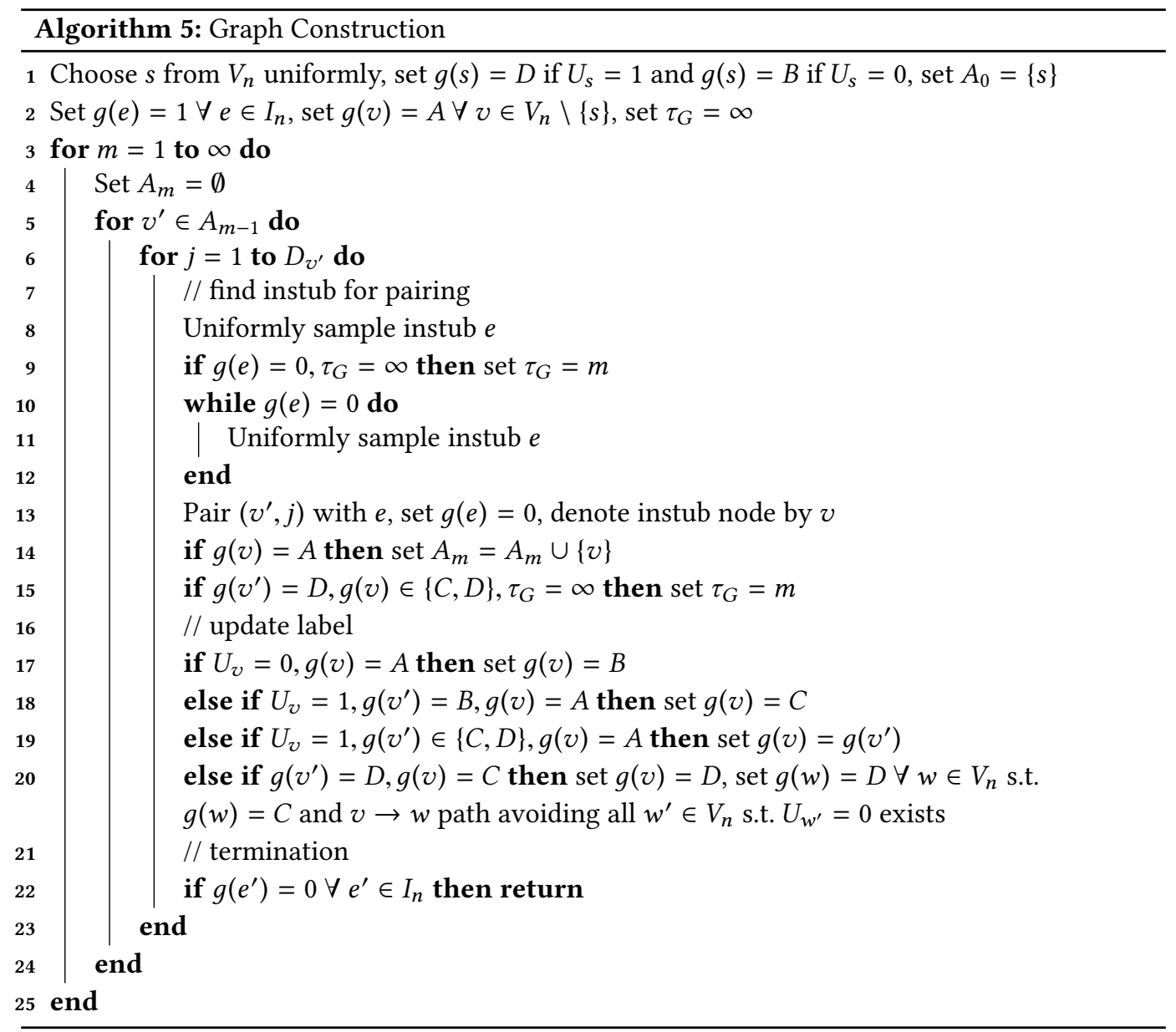

First, recall the error bound in Lemma 3 depends only on the $m$-step neighborhood out of $s$. Hence, a typical approach to analyzing the bound would be to argue that this neighborhood is treelike, and then to analyze the bound on a related tree. However, this is more than we require. To see this, we return to the example from Figure 7. As argued previously, the error bound only depends on the orange dashed subgraph. Hence, the related tree we construct will (roughly speaking) only contain this subgraph, i.e. rather than require the entire $m$-step neighborhood to be treelike, we only require the $m$-step neighborhood of label $D$ nodes to be treelike.

This discussion also helps explain the variable $\tau_{G}$ in Algorithm 5. Observe that we set $\tau_{G}=m$ if we pair an outstub of $v^{\prime} \in A_{m-1}$ with an instub of $v$, where $g\left(v^{\prime}\right)=D$ and $g(v) \in\{C, D\}$ (Line 15 in Algorithm 5). As shown in Figure 8, these events (may) destroy the tree structure of the label $D$ subgraph. Additionally, we set $\tau_{G}=m$ if we sample an instub that has already been paired while attempting to pair an outstub of $v^{\prime} \in A_{m-1}$ (Line 9 in Algorithm 5). This is to ensure nodes have i.i.d. attributes $\left(N_{v}, D_{v}, U_{v}\right)$, as nodes in the tree construction will have.

This intuition motivates our tree construction. In the tree construction, we begin with a root node denoted by $\phi$, and we assign attributes $\left(N_{\phi}, D_{\phi}, U_{\phi}\right)$. Here $N_{\phi}$ is the number of instubs of $\phi$, all of which will remain unpaired for the duration of the algorithm (so that the tree structure 


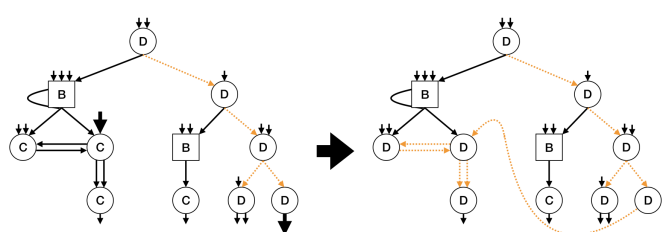

(a) Enlarged outstub belongs to label $D$ node, enlarged instub belongs to label $C$ node.

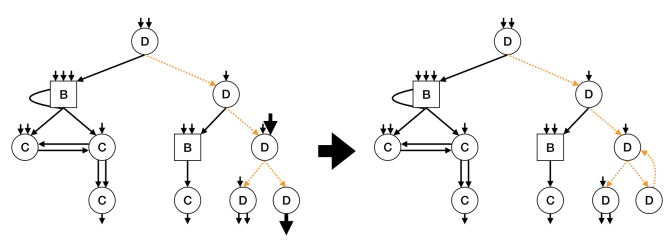

(b) Enlarged outstub belongs to label $D$ node, enlarged instub belongs to label $D$ node.

Fig. 8. If the enlarged instub is sampled for pairing with the enlarged outstub at left, then, after updating node labels, the orange dashed subgraph of label $D$ nodes at right is no longer a tree.

is maintained); $D_{\phi}$ is the number of offspring of $\phi$; and $U_{\phi}=1$. To each offspring of $\phi$, denoted $1,2, \ldots, D_{\phi}$, we assign attributes $\left(N_{i}, D_{i}, U_{i}\right)$. Here $N_{i}$ denotes the number of instubs of $i$; one of these is paired with the $i$-th outstub of $\phi$, while the other $N_{i}-1$ remain unpaired (again, to preserve the tree structure). Furthermore, unlike the root node, node $i$ receives $D_{i}$ offspring only if $U_{i}=1$; otherwise, the outstubs remain unpaired. This is explained by Figure 7 , since only the orange dashed subgraph affects the quantity of interest.

The set of nodes $1,2, \ldots, D_{\phi}$ is denoted by $\hat{A}_{1}$. In general, we denote by $\hat{A}_{m}$ the $m$-th generation of the tree, i.e. the set of nodes at distance $m$ from the root node. The generic node in $\hat{A}_{m}, m>1$ is denoted by $\mathbf{i}$, where $\mathbf{i}=\left(i_{1}, i_{2}, \ldots, i_{m}\right)$ is an ordered list of natural numbers that traces the unique path from $\phi$ to $\mathbf{i}$ : specifically, this path is $\phi \in \hat{A}_{0}, i_{1} \in \hat{A}_{1},\left(i_{1}, i_{2}\right) \in \hat{A}_{2}, \ldots, \mathbf{i} \in \hat{A}_{m}$. The offspring of $\mathbf{i}$ (assuming $\left.U_{\mathbf{i}}=1\right)$ are denoted by $\{(\mathbf{i}, j)\}_{j=1}^{D_{\mathrm{i}}}$, where $(\mathbf{i}, j)=\left(i_{1}, i_{2}, \ldots, i_{m}, j\right)$ is the concatenation operation.

To assign attributes, we require two (empirical) distributions: given $\left\{N_{h}, D_{h}, U_{h}\right\}_{h=1}^{n}$, we define $f_{n}: \mathbb{N} \times \mathbb{N} \times\{0,1\} \rightarrow[0,1]$ and $f_{n}^{*}: \mathbb{N} \times \mathbb{N} \rightarrow[0,1]$ according to (21). Note that $f_{n}$ is the distribution of node attributes for nodes sampled proportional to in-degree, whereas $f_{n}^{*}$ is the distribution of node attributes for nodes sampled uniformly at random from $V_{n} \backslash K_{n}$. Because non-root nodes are sampled proportional to in-degree in the graph construction (until an edge must be resampled, i.e. until we set $\tau_{G}=m$ ), non-root node attributes are sampled from $f_{n}$ in the tree construction. Similarly, since the first node is sampled uniformly from $V_{n} \backslash K_{n}$ in the case of interest of the graph construction, root node attributes attributes are sampled from $f_{n}^{*}$ in the tree construction.

$$
f_{n}(i, j, k)=\sum_{h=1}^{n} \frac{N_{h}}{L_{n}} 1\left(N_{h}=i, D_{h}=j, U_{h}=k\right), \quad f_{n}^{*}(i, j)=\sum_{h=1}^{n} \frac{U_{h}}{\sum_{h^{\prime}=1}^{n} U_{h^{\prime}}} 1\left(N_{h}=i, D_{h}=j\right) .
$$

The tree construction is given formally in Algorithm 6 . We denote by $\hat{G}_{n}=\left(\hat{V}_{n}, \hat{E}_{n}\right)$ the resulting tree. Note the tree construction continues indefinitely, so the subscript $n$ does not refer to the number of nodes in the tree; rather, it refers to the length of the sequence $\left\{N_{h}, D_{h}, U_{h}\right\}_{h=1}^{n}$ from which the distributions $f_{n}, f_{n}^{*}$ are defined. Finally, in Figure 9, we show an example of the tree construction, which corresponds to the graph construction of Figures 7 (i.e. the dashed orange subgraph has the same structure).

Having defined the tree construction, we now define the aforementioned quantity that follows the same distribution as the error bound in Lemma 3. Specifically, we define $\hat{\mu}_{\phi}$ recursively as

$$
\hat{\mu}_{\phi}(\phi)=1, \quad \hat{\mu}_{\phi}((\mathbf{i}, j))=\hat{\mu}_{\phi}(\mathbf{i}) \frac{\left(1-\alpha_{n}\right) U_{\mathbf{i}}}{D_{\mathbf{i}}},(\mathbf{i}, j) \in \hat{A}_{l}, l>0,
$$




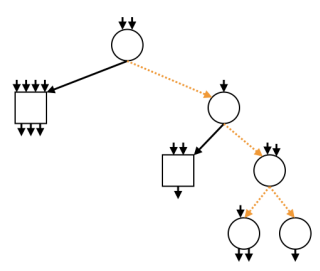

Fig. 9. Branching process after three generations, corresponding to the example graph from Figure 7. In particular, the orange dashed subgraphs are identical.

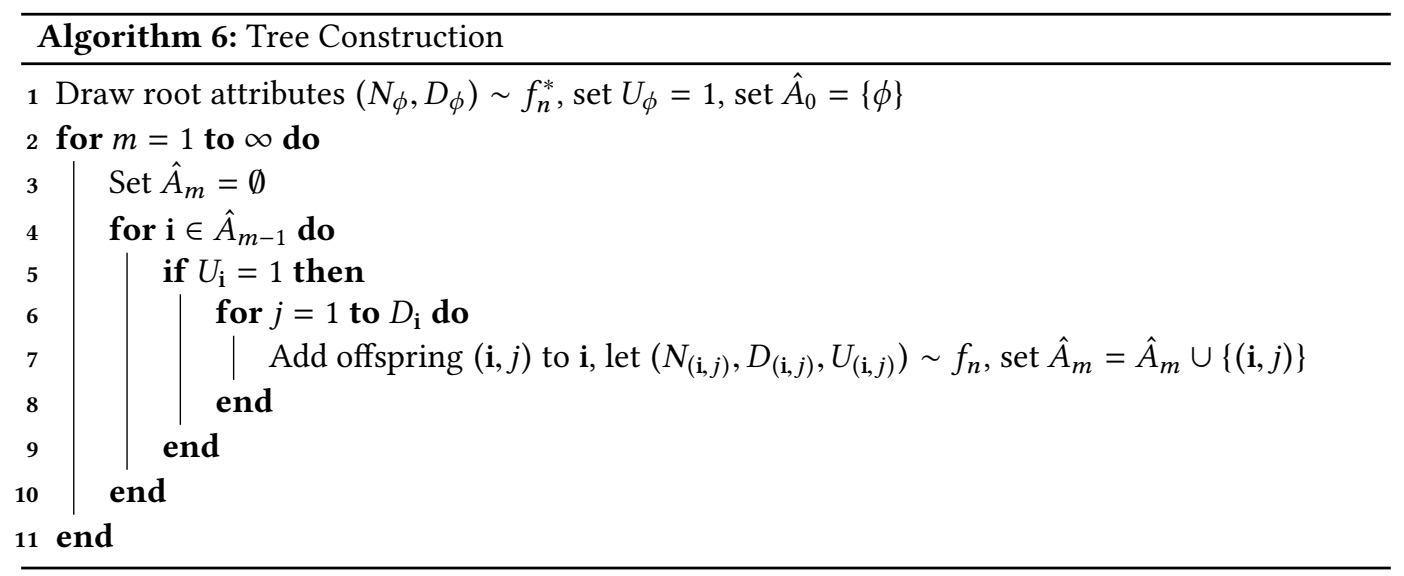

where (by convention), $\mathbf{i}=\phi$ when $(\mathbf{i}, j)=i_{1} \in \mathbb{N}$, i.e. when $(\mathbf{i}, j) \in \hat{A}_{1}$. Note that (22) is the same as (19) but computed on the tree $\hat{G}_{n}$; because there is a unique path from $\phi$ to $\mathbf{i}$ for each $\mathbf{i} \in \hat{V}_{n}$, this recursive definition will be more convenient.

We next state Lemma 4, whose proof is deferred to Appendix D.3. The proof formalizes the preceding intuition, that when $\tau_{G}>m$, the error bound from Lemma 3 is computed on a treelike subgraph and therefore follows the distribution of the analogous tree quantity.

Lemma 4. For any $m \in \mathbb{N}$,

$$
\mu_{s}^{(m)}\left(V_{n} \backslash K_{n}\right) \mid\left\{\tau_{G}>m, U_{s}=1\right\} \stackrel{\mathcal{D}}{=} \sum_{j=0}^{m} \sum_{\mathbf{i} \in \hat{A}_{j}} U_{\mathbf{i}} \hat{\mu}_{\phi}(\mathbf{i}),
$$

where $\stackrel{\mathcal{D}}{=}$ denotes equality in distribution.

Proof. See Appendix D.3.

We can now explain the remainder of our approach to proving the lemma. Using Lemmas 3 and 4, and noting that, by definition, $\sum_{\mathbf{i} \in \hat{A}_{0}} U_{\mathrm{i}} \hat{\mu}_{\phi}(\mathbf{i})=U_{\phi} \hat{\mu}_{\phi}(\phi)=1$, we have

$$
\begin{aligned}
\mathbb{P}\left[B_{s}\left(K_{n}, \epsilon\right) \mid U_{s}=1\right] & \leq \mathbb{P}\left[\alpha_{n}\left(\mu_{s}^{(m-1)}\left(V_{n} \backslash K_{n}\right)-1\right)+e_{s}^{\mathrm{T}}(1-\alpha)^{m} \tilde{P}^{m} e_{V_{n} \backslash K_{n}} \geq \epsilon \mid U_{s}=1\right] \\
& \leq \mathbb{P}\left[\tau_{G} \leq m \mid U_{s}=1\right]+\mathbb{P}\left[\alpha_{n} \sum_{j=1}^{m-1} \sum_{\mathbf{i} \in \hat{A}_{j}} U_{\mathbf{i}} \hat{\mu}_{\phi}(\mathbf{i})+\sum_{\mathbf{i} \in \hat{A}_{m}} U_{\mathbf{i}} \hat{\mu}_{\phi}(\mathbf{i}) \geq \epsilon\right] .
\end{aligned}
$$


Hence, our approach to bounding the probability of (3) will be to further bound the two summands in (23). Since (23) holds for any $m \in \mathbb{N}$, our final step will be to choose $m$ to optimize the sum of these bounds. In particular, we will choose $m$ to balance the two bounds. This is because the summands are increasing and decreasing in $m$, respectively.

\section{A.3 Coupling failure (Step 3)}

Our bound for the first summand in (23) is given in Lemma 5. This result is similar to Lemma 5.4 of [21], and our proof follows a similar approach. However, Assumption 1 is different than the assumption required for the result in [21]. This difference arises because the result in [21] requires the entire $m$-step neighborhood to be treelike, while we only require the $m$-step neighborhood of label $D$ nodes to be treelike. This allows us to relax the assumption from [21], which requires $\sum_{h=1}^{n} N_{h}^{2} / n$ to converge to a finite limit; we only require $\sum_{h=1}^{n} N_{h}^{2} U_{h} / n$ to converge. In fact, the example degree sequence presented in Section 7.3 satisfies

$$
\mathbb{E}\left[N_{h}^{2} U_{h}\right]=O(1), \quad \mathbb{E}\left[N_{h}^{2}\right]=O\left(n^{l_{2}}\right),
$$

where $l_{2}>0$. Hence, there are sequences for which the existing lemma from [21] does not apply, but for which our version does apply. This is why we do not directly use the lemma from [21].

Lemma 5. Given Assumption 1, for any $m_{n} \rightarrow \infty$ as $n \rightarrow \infty$ s.t. $m_{n}=O\left(n^{\gamma}\right)$, we have

$$
\mathbb{P}\left[\tau_{G} \leq m_{n} \mid U_{s}=1\right]=O\left(n^{-\delta}+\zeta^{m_{n}} / \sqrt{n}\right),
$$

where $\gamma, \delta, \zeta$ are defined in Assumption 1.

Proof. See Appendix D.4.

\section{A.4 Tail bound on branching process quantity (Step 4)}

Our final step is to bound the second summand in (23). Our approach is to bound the probability that either $\alpha_{n} \sum_{j=1}^{m-1} \sum_{\mathbf{i} \in \hat{A}_{j}} U_{\mathbf{i}} \hat{\mu}_{\phi}(\mathbf{i})$ or $\sum_{\mathbf{i} \in \hat{A}_{m}} U_{\mathbf{i}} \hat{\mu}_{\phi}(\mathbf{i})$ exceeds $\epsilon / 2$. For the first term, the recursive definition of $\hat{\mu}_{\phi}$ yields a martingale structure that allows us to use an approach similar to the method of bounded differences (see, for example, Section 5.4 of [24]). The second term arises from the tail of the $m$-step neighborhood approximation from Appendix A.1, and it is not a sum of terms; hence, its expected value decays geometrically fast in $m$, so Markov's inequality suffices.

Lemma 6. Given Assumption 1, for any $\epsilon>0$, any $m_{n} \rightarrow \infty$ as $n \rightarrow \infty$ s.t. $m_{n}=O\left(n^{\gamma}\right)$, and any $\alpha_{n} \rightarrow 0$ as $n \rightarrow \infty$, we have

$$
\mathbb{P}\left[\alpha_{n} \sum_{j=1}^{m-1} \sum_{\mathbf{i} \in \hat{A}_{j}} U_{\mathbf{i}} \hat{\mu}_{\phi}(\mathbf{i})+\sum_{\mathbf{i} \in \hat{A}_{m}} U_{\mathbf{i}} \hat{\mu}_{\phi}(\mathbf{i}) \geq \epsilon\right]=O\left(n^{-\delta}+p^{m_{n}}+e^{-((1-p) \epsilon)^{2} /\left(2 \alpha_{n}\right)}\right),
$$

where $p, \delta$ are defined in Assumption 1.

Proof. See Appendix D.5.

\section{A.5 Completing the proof of Lemma 1}

Finally, we can combine the results of this section to prove Lemma 1. First, we substitute the results of Lemmas 5 and 6 into (23) to obtain (when Assumption 1 holds)

$$
\mathbb{P}\left[B_{s}\left(K_{n}, \epsilon\right) \mid U_{s}=1\right]=O\left(n^{-\delta}+\frac{\zeta^{m_{n}}}{\sqrt{n}}+p^{m_{n}}+e^{-((1-p) \epsilon)^{2} /\left(2 \alpha_{n}\right)}\right) .
$$


Next, choose $m_{n}=\frac{\log n}{2 \log (\zeta / p)}$ to equate the middle two terms, i.e.

$$
\frac{\zeta^{m_{n}}}{\sqrt{n}}=p^{m_{n}}=n^{-\log (1 / p) /(2 \log (\zeta / p))}
$$

For the third term, we take $\alpha_{n}=\rho \log (1 / \tau) \log \zeta / \log n$ as defined in Proposition 1 to obtain

$$
\exp \left(-\frac{((1-p) \epsilon)^{2}}{2 \alpha_{n}}\right)=n^{-((1-p) \epsilon)^{2} /(2 \rho \log (1 / \tau) \log \zeta)} .
$$

Hence, we ultimately obtain

$$
\mathbb{P}\left[B_{s}\left(K_{n}, \epsilon\right) \mid U_{s}=1\right]=O\left(n^{-c(\epsilon)}\right),
$$

where $c(\epsilon)$ is defined as in the statement of the lemma.

\section{B EXPERIMENTAL DETAILS}

\section{B.1 Dataset details}

The following table shows details of the datasets used for experiments in Section 6. All datasets are available from the Stanford Network Analysis Platform [37]. The $\alpha_{n}$ values shown are used for all experiments conducted on the corresponding graph. We note that, while these are smaller than $\alpha_{n}$ values typically used, they are the same order of magnitude $\left(\alpha_{n}=0.15\right.$ is a common choice in the literature). Finally, we note that the datasets with prefix web- are partial web crawls; those with prefix soc- are social networks.

\begin{tabular}{|l|l|c|c|}
\hline Dataset & $n$ & $L_{n}$ & $\alpha_{n}=1 / \log n$ \\
\hline soc-LiveJournal1 & 4847571 & 68993773 & 0.065 \\
\hline soc-pokec & 1632803 & 30622564 & 0.070 \\
\hline web-Google & 875713 & 5105039 & 0.073 \\
\hline web-BerkStan & 685230 & 7600595 & 0.074 \\
\hline web-Stanford & 281903 & 2312497 & 0.080 \\
\hline
\end{tabular}

\section{B.2 Scheme to bound estimation error}

To bound $\left\|\pi_{v}-\left(\alpha_{n} e_{v}^{\top}+\sum_{k \in K_{n}} \beta_{v}(k) \pi_{k}\right)\right\|_{1}$, where $\beta_{v}(k)$ are defined in (8), we employ a power iteration scheme: we initialize $x_{v}^{(0)}=e_{v}^{\top}$, and given $x_{v}^{(i-1)}$ for $i \geq 1$, we set

$$
x_{v}^{(i)}=\alpha_{n} e_{v}^{\top}+\left(1-\alpha_{n}\right) x_{v}^{(i-1)} \tilde{P},
$$

where $\tilde{P}$ is defined in (6). We claim

$$
x_{v}^{(i)}=\alpha_{n} \mu_{v}^{(i-1)}+\left(1-\alpha_{n}\right)^{i} e_{v}^{\top} \tilde{P}^{i}
$$

where $\mu_{v}^{(i-1)}=e_{v}^{\top} \sum_{j=0}^{i-1}\left(1-\alpha_{n}\right)^{j} \tilde{P}^{j}$ (as in Appendix A-D). (24) is easily proven inductively: the base of induction holds by definition; assuming true for $i-1$, we have

$$
\begin{aligned}
x_{v}^{(i)} & =\alpha_{n} e_{v}^{\top}+\left(1-\alpha_{n}\right)\left(\alpha_{n} \mu_{v}^{(i-2)}+\left(1-\alpha_{n}\right)^{i-1} e_{v}^{\top} \tilde{P}^{i-1}\right) \tilde{P} \\
& =\alpha_{n} e_{v}^{\top}+\alpha_{n} \sum_{j=1}^{i-1}\left(1-\alpha_{n}\right)^{j} \tilde{P}^{j}+\left(1-\alpha_{n}\right)^{i} e_{v}^{\top} \tilde{P}^{i}=\alpha_{n} \mu_{v}^{(i-1)}+\left(1-\alpha_{n}\right)^{i} e_{v}^{\top} \tilde{P}^{i}
\end{aligned}
$$


as claimed. Now by Lemma 3 in Appendix A, for any $i \in \mathbb{N}$ we obtain the following bound:

$$
\begin{aligned}
\left\|\pi_{v}-\left(\alpha_{n} e_{v}^{\top}+\sum_{k \in K_{n}} \beta_{v}(k) \pi_{k}\right)\right\|_{1} & \leq \alpha_{n} \mu_{v}^{(i-1)}\left(V_{n} \backslash K_{n}\right)+\left(1-\alpha_{n}\right)^{i} e_{v}^{\top} \tilde{P}^{i} e_{V_{n} \backslash K_{n}}-\alpha_{n} \\
& =x_{v}^{(i)}\left(V_{n} \backslash K_{n}\right)-\alpha_{n} .
\end{aligned}
$$

From this bound, we can prove two other claims from Section 5. First, we note

$$
x_{v}^{(i)}\left(V_{n} \backslash K_{n}\right)=\alpha_{n} e_{v}^{\top} \sum_{j=0}^{i-1}\left(1-\alpha_{n}\right)^{j} \tilde{P}^{j}+\left(1-\alpha_{n}\right)^{i} e_{v}^{\top} \tilde{P}^{i} e_{V_{n} \backslash K_{n}} \leq \alpha_{n} \sum_{j=0}^{i-1}\left(1-\alpha_{n}\right)^{j}+\left(1-\alpha_{n}\right)^{i}=1
$$

where the inequality follows since $\tilde{P}$ is nonnegative with row sums bounded by 1 . Hence, from (26), the estimation error is bounded by $\left(1-\alpha_{n}\right)$ (as claimed in Section 5). Next, suppose $v \in V_{n, 0}$, with $V_{n, 0}$ given by (14). Then $e_{v}^{\top} \tilde{P}^{j} e_{V_{n} \backslash K_{n}}=0$, so $x_{v}^{(i)}\left(V_{n} \backslash K_{n}\right)=\alpha_{n}$, and the estimation error is zero (as claimed in Section 6).

We can also bound the gap in the inequality (25): use (57) in Appendix D.2 and (25) to write

$$
\begin{aligned}
& \left\|\pi_{v}-\left(\alpha_{n} e_{v}^{\top}+\sum_{k \in K_{n}} \beta_{v}(k) \pi_{k}\right)\right\|_{1}-\left(x_{v}^{(i)}\left(V_{n} \backslash K_{n}\right)-\alpha_{n}\right) \\
& =\left(\alpha_{n} e_{v}^{\top} \sum_{j=0}^{\infty}\left(1-\alpha_{n}\right)^{i} \tilde{P}^{i} e_{V_{n} \backslash K_{n}}-\alpha_{n}\right)-\left(x_{v}^{(i)}\left(V_{n} \backslash K_{n}\right)-\alpha_{n}\right) \\
& =\alpha_{n} \mu_{v}^{(i-1)}\left(V_{n} \backslash K_{n}\right)+\alpha_{n} e_{v}^{\top} \sum_{j=i}^{\infty}\left(1-\alpha_{n}\right)^{i} \tilde{P}^{i} e_{V_{n} \backslash K_{n}}-x_{v}^{(i)}\left(V_{n} \backslash K_{n}\right) \\
& =\alpha_{n} e_{v}^{\top} \sum_{j=i}^{\infty}\left(1-\alpha_{n}\right)^{i} \tilde{P}^{i} e_{V_{n} \backslash K_{n}}-\left(1-\alpha_{n}\right)^{i} e_{v}^{\top} \tilde{P}^{i} e_{V_{n} \backslash K_{n}} \geq-\left(1-\alpha_{n}\right)^{i},
\end{aligned}
$$

where the inequality holds by dropping a nonnegative term and since $e_{v}^{\top} \tilde{P}^{i} e_{V_{n} \backslash K_{n}} \leq 1$. Hence, if we let $i^{*} \geq \log _{\left(1-\alpha_{n}\right)}(t o l)$ for some desired tolerance $t o l$, the bound $x_{v}^{\left(i^{*}\right)}\left(V_{n} \backslash K_{n}\right)-\alpha_{n}$ is tight within additive error tol. (For all experiments, we set $t o l=0.05$.)

To bound average error across $V_{n} \backslash K_{n}$, we instead use

$$
x_{V_{n} \backslash K_{n}}^{(0)}=\frac{e_{V_{n} \backslash K_{n}}^{\top}}{\left|V_{n} \backslash K_{n}\right|}, \quad x_{V_{n} \backslash K_{n}}^{(i)}=\alpha_{n} \frac{e_{V_{n} \backslash K_{n}}^{\top}}{\left|V_{n} \backslash K_{n}\right|}+\left(1-\alpha_{n}\right) x_{V_{n} \backslash K_{n}}^{(i-1)} \tilde{P} .
$$

Note $x_{V_{n} \backslash K_{n}}^{(i)}=\frac{1}{\left|V_{n} \backslash K_{n}\right|} \sum_{v \in V_{n} \backslash K_{n}} x_{v}^{(i)}$ when $i=0$ by definition; assuming true for general $i-1$,

$$
\begin{aligned}
x_{V_{n} \backslash K_{n}}^{(i)} & =\alpha_{n} \frac{e_{V_{n} \backslash K_{n}}^{\top}}{\left|V_{n} \backslash K_{n}\right|}+\left(1-\alpha_{n}\right)\left(\frac{1}{\left|V_{n} \backslash K_{n}\right|} \sum_{v \in V_{n} \backslash K_{n}} x_{v}^{(i-1)}\right) \tilde{P} \\
& =\frac{1}{\left|V_{n} \backslash K_{n}\right|} \sum_{v \in V_{n} \backslash K_{n}}\left(\alpha_{n} e_{v}^{\top}+\left(1-\alpha_{n}\right) x_{v}^{(i-1)} \tilde{P}\right)=\frac{1}{\left|V_{n} \backslash K_{n}\right|} \sum_{v \in V_{n} \backslash K_{n}} x_{v}^{(i)},
\end{aligned}
$$

i.e. $x_{V_{n} \backslash K_{n}}^{(i)}=\frac{1}{\left|V_{n} \backslash K_{n}\right|} \sum_{v \in V_{n} \backslash K_{n}} x_{v}^{(i)} \forall i \in \mathbb{N}$. It follows from above that

$$
\frac{1}{\left|V_{n} \backslash K_{n}\right|} \sum_{v \in V_{n} \backslash K_{n}}\left\|\pi_{v}-\left(\alpha_{n} e_{v}^{\top}+\sum_{k \in K_{n}} \beta_{v}(k) \pi_{k}\right)\right\|_{1} \leq x_{V_{n} \backslash K_{n}}^{(i)}\left(V_{n} \backslash K_{n}\right)-\alpha_{n},
$$




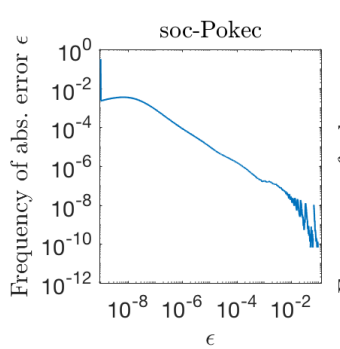

(a) soc-Pokec (social network)
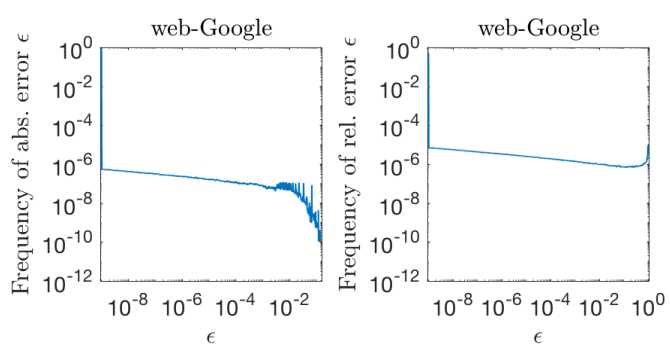

(b) web-Google (partial web crawl)

Fig. 10. Detailed error analyses

which is the average error bound we compute for Figures $2 \mathrm{a}, 2 \mathrm{~b}$, and 3 . The argument above also implies this bound is tight within $t o l$ when $i^{*} \geq \log _{\left(1-\alpha_{n}\right)}(t o l)$.

\section{B.3 Details on Figure 1 experiment}

In addition to the histograms of $l_{1}$ error shown in Figure 1a, we include a more detailed set of plots for the same experiment. Specifically, we estimate the error $\left|\alpha_{n} e_{v}^{\top}(w)+\sum_{k \in K_{n}} \beta_{v}(k) \pi_{k}(w)\right|$ as $x_{v}^{(i)}(w)-\alpha_{n} 1(w=v)$ (where $x_{v}^{(i)}$ is defined in Appendix B.2), for each $w \in V_{n} \backslash K_{n}$, and for each $v$ in a subset of $V_{n} \backslash K_{n}$ of size $\approx 10^{4}$. (These $v$ were chosen uniformly from nodes with average error $\in(0.08,0.25)$, which corresponds to the regime of linear decay in Figure 1.) We also estimate the relative error, i.e. the ratio of this absolute error to an estimate of $\pi_{v}(w)$, for the same set of $(v, w)$. The estimate of $\pi_{v}$ is computed using the same power iteration scheme in Appendix B.2, but replacing $\tilde{P}$ with $P$. Note this gives a lower bound on the true value of $\pi_{v}(w)$, thereby upper bounding relative error. Unfortunately, we cannot compute this relative error estimate when the estimate of $\pi_{v}(w)$ is zero; this occurred for only $10 \%$ of $(v, w)$ pairs considered. Finally, for both absolute and relative error, we compute the number of error values lying in log-spaced bins and divide these values by $n$ to estimate the frequency of each error value. (We add values lying beyond the first and last bin edges to the first and last bins, respectively.)

Results are shown for the soc-Pokec dataset in Figure 10a. (We note the spikes at left occur due to values lying beyond the first bin edge.) As an illustration for absolute error, the frequency of values above $10^{-3}$ was $\approx 10^{-5}$, i.e. the vast majority of nodes had estimated absolute error below $10^{-3}$. To illustrate the relative error, the frequency of values above 0.2 was $\approx 0.09$, i.e. over $90 \%$ of nodes had estimated relative error below 0.2 . The results for web-Google are shown in Figure $10 \mathrm{~b}$. For absolute error, the frequency above $10^{-3}$ was again $\approx 10^{-5}$; for relative error, again over $90 \%$ of nodes had error below 0.2 .

\section{B.4 Geometric interpretation of Theorem 1}

In Figure 11, we show a graphical representation of Theorem 1 similar to Figure 6 in Section 7.5, but here using actual PPR vectors. For these plots, $G_{n}$ is a DCM with in-degrees following a power law with exponent 2 and out-degrees generated as in Algorithm 4 from Section 7.3. The dots are projections of the $n$-dimensional vectors $\left\{\pi_{v}\right\}_{v \in V_{n} \backslash K_{n}}$ into 2D space; specifically, the $v$-th dot is at $\left(\pi_{v}\left(v_{1}\right), \pi_{v}\left(v_{2}\right)\right)$, where $v_{1}$ is the node of highest in-degree and $v_{2}$ is the node of second-highest in-degree. Red and green, respectively, correspond to those $v$ for which $B_{v}\left(K_{n}, \epsilon\right)$ holds and fails, respectively, with $K_{n}$ chosen as the $\sqrt{n}$ nodes of highest in-degree and $\epsilon=0.2$. Finally, the region outlined in blue is the convex hull of $\left\{\left(\pi_{k}\left(v_{1}\right), \pi_{k}\left(v_{2}\right)\right)\right\}_{k \in K_{n}}$. 

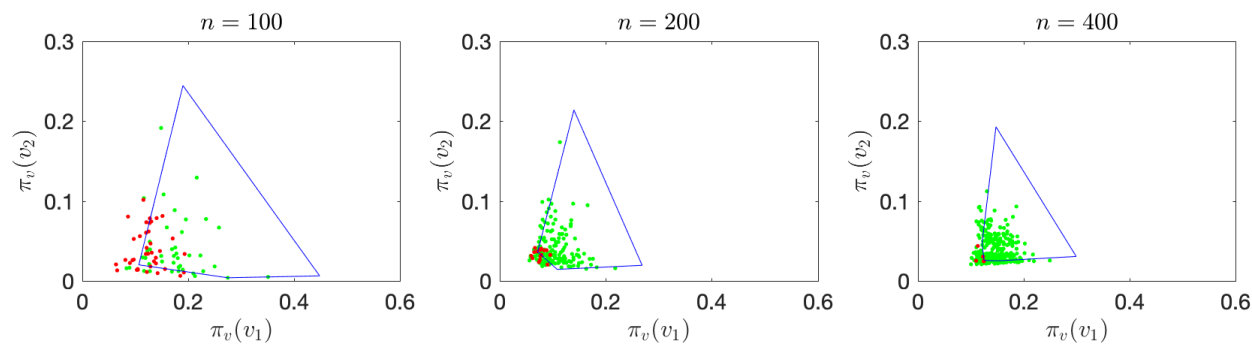

Fig. 11. A geometric illustration similar to Figure 6 in Section 7.5, but here using actual PPR vectors.

Note that, as $n$ grows, a larger fraction of dots fall near or within the blue outlined region, and the area of this region decreases as $n$ grows, as in Figure 6. However, the dichotomy of green dots lying inside the region and red dots lying outside the region is much less clear than in Figure 6. This is in part because the projection $\pi_{v} \mapsto\left(\pi_{v}\left(v_{1}\right), \pi_{v}\left(v_{2}\right)\right)$ is not $l_{1}$ distance-preserving. Instead, green and red dots exhibit a different distinction in Figure 11: roughly speaking, red dots lie closer to the bottom left of each plot, while green dots lie closer to the top right. This is because $v_{1}, v_{2} \in K_{n}$ by definition of $v_{1}, v_{2}$ and choice of $K_{n}$; hence, dots near the top right are "close" in the graph to at least two elements of $K_{n}$, which (at a high level) means their PPR vectors are well-approximated as linear combinations of $\left\{\pi_{k}\right\}_{k \in K_{n}}$.

\section{ALGORITHMIC COMPARISONS}

\section{C.1 Nonnegative matrix factorization}

As discussed in Section 5.2, our algorithm can be viewed as a variant of nonnegative matrix factorization (NMF). To explain this, we will assume for simplicity that nodes are labeled such that $K_{n}=\left\{1, \ldots,\left|K_{n}\right|\right\}$ and $B_{v}\left(K_{n}, \epsilon / 14\right)$ holds $\forall v \in\left\{\left|K_{n}\right|+1, \ldots, \Delta\left(K_{n}, \epsilon / 14\right)\right\}$ (i.e. for such $v, \pi_{v}$ is far from a linear combination of $\left.\left\{\pi_{k}\right\}_{k \in K_{n}}\right)$. Thus, for $v \leq \Delta\left(K_{n}, \epsilon / 14\right)$, the estimate $\hat{\pi}_{v}$ is computed via Approx-PageRank, while for $v>\Delta\left(K_{n}, \epsilon / 14\right), \hat{\pi}_{v}$ is computed as

$$
\hat{\pi}_{v}=\alpha_{n} e_{v}^{\top}+\frac{\sum_{k \in K_{n}} \hat{\tilde{\pi}}_{v}(k) \hat{\pi}_{k}}{\alpha_{n}+\left(1-\alpha_{n}\right) \hat{\tilde{\pi}}_{v}\left(K_{n}\right)} .
$$

Hence, Algorithm 1 outputs $\alpha_{n} I+W H$, where $W \in \mathbb{R}^{n \times \Delta\left(K_{n}, \epsilon / 14\right)}, H \in \mathbb{R}^{\Delta\left(K_{n}, \epsilon / 14\right) \times n}$ have rows

$$
\begin{aligned}
& W(v,:)=\left\{\begin{array}{ll}
e_{v}^{\top}, & v \leq \Delta\left(K_{n}, \epsilon / 14\right) \\
\frac{\left[\hat{\pi}_{v}(1) \cdots \hat{\tilde{\pi}}_{v}\left(\left|K_{n}\right|\right) 0 \cdots 0\right]}{\alpha_{n}+\left(1-\alpha_{n}\right) \tilde{\pi}_{v}\left(K_{n}\right)}, & v>\Delta\left(K_{n}, \epsilon / 14\right)
\end{array},\right. \\
& H(v,:)=\hat{\pi}_{v}-\alpha_{n} e_{v}^{\top} .
\end{aligned}
$$

In short, our algorithm computes matrices $W$ and $H$ such that

$$
\left\|\left(\Pi_{n}-\alpha_{n} I\right)-W H\right\|_{\infty}<\epsilon
$$

which is the NMF-like objective function discussed in Section 5.2.

As mentioned in Section 5.2, our algorithm offers several advantages over typical NMF algorithms. First, it is provably accurate (for general graphs) and provably efficient (for the DCM). Second, it is adaptive in terms of the dimensions of $W$ and $H$ : while standard NMF algorithms assume $W \in \mathbb{R}^{n \times r}, H \in \mathbb{R}^{r \times n}$ a priori for some $r$ (typically $r \ll n$ to obtain a low rank estimate), our algorithm determines at runtime which $\pi_{v}, v \notin K_{n}$ cannot be approximated as linear combinations of $\left\{\pi_{k}\right\}_{k \in K_{n}}$ and adjusts $r$ to account for this (ultimately yielding $r=\Delta\left(K_{n}, \epsilon / 14\right)$ as above). Additionally, off-the-shelf NMF algorithms are difficult to adapt to our setting for several reasons. 
First, our objective function uses the non-differentiable norm $\|\cdot\|_{\infty}$, so we cannot compute the gradients needed for standard NMF. Second, even if our objective was differentiable, it involves the unknown matrix $\Pi_{n}$, again rendering gradient calculations impossible (typically, NMF aims to find $W, H$ so as to minimize $\|X-W H\|$ for some known matrix $X)$.

To overcome these issues, one could instead use the objective function

$$
J(W, H)=\min _{W, H \geq 0} \frac{1}{2}\left\|\alpha_{n} I-W H\left(I-\left(1-\alpha_{n}\right) P\right)\right\|_{F}^{2} .
$$

Here we have used the differentiable norm $\|\cdot\|_{F}$ and removed the unknown matrix $\Pi_{n}$ from the objective function. Additionally, the form of this new objective function is motivated by (4), which shows $\Pi_{n}=\alpha_{n}\left(I-\left(1-\alpha_{n}\right) P\right)^{-1}$; hence, $J(W, H)=0$ when $W H=\Pi_{n}$. With this objective function, the multiplicative update rule for NMF from [35] can be applied, which is

$$
W \leftarrow W \nabla_{W}^{-} / \nabla_{W}^{+}, \quad H \leftarrow H \nabla_{H}^{+} / \nabla_{H}^{-},
$$

where the multiplication and division is elementwise, and where $\nabla_{W}^{-}$and $\nabla_{W}^{+}$are the negative and positive parts of the gradient of $J$ with respect to $W\left(\nabla_{H}^{-}, \nabla_{H}^{+}\right.$are defined analogously). However, we claim that this method will perform worse than our algorithm. To see why, we define $Y=$ $\left(I-\left(1-\alpha_{n}\right) P\right)$ and note

$$
\begin{aligned}
& \nabla_{W}=-\alpha_{n} Y^{\top} H^{\top}+W H Y Y^{\top} H^{\mathrm{T}}, \\
& \nabla_{H}=-\alpha_{n} W^{\mathrm{\top}} Y^{\mathrm{\top}}+W^{\mathrm{\top}} W H Y Y^{\top} .
\end{aligned}
$$

Here (for example) multiplying $W^{\top}$ by $W H Y Y^{\top}$ to compute $\nabla_{H}$ at the first iteration has complexity $O\left(n^{2} r\right)$, assuming $W \in \mathbb{R}^{n \times r}$ and $H \in \mathbb{R}^{r \times n} .^{9}$ In short, the first iteration of this NMF algorithm has higher complexity than our entire algorithm. We also note [51] provides algorithms similar to those from [35], but tailored to stochastic matrices (such as $\Pi_{n}$ ); however, these still involve computation of $\nabla_{W}, \nabla_{H}$ and a multiplicative update, so the same issue remains.

\section{C.2 Representation learning}

In Section 5.2, we also noted a connection between our algorithm and the representation learning scheme from [30]. At a high level, the latter proceeds in two stages. The first stage learns certain parameters for the second stage via stochastic gradient descent; the second stage (Algorithm 1 in [30]) is described informally as Algorithm 7 here. Roughly speaking, this second stage begins by representing each node with a given feature vector, then updates this representation based on features of neighbors (after one iteration), neighbors of neighbors (after two iterations), etc.

There are some immediate issues with applying Algorithm 7 to our setting. First, it relies on given feature vectors, derived from e.g. text data pertaining to each node; our algorithm assumes only the graph structure is known. Second, it applies to undirected graphs; we have assumed a directed graph throughout the paper. However, setting these issues aside, we next discuss two more subtle issues with adapting this algorithm to PPR estimation.

We first consider the most immediate adaptation of Algorithm 7 to our setting, which we present as Algorithm 8. Here we let feature vectors simply be point masses on each node (Line 1), we let the aggregate function be a simple average (Line 4), and we update $h_{v}^{k}$ as a weighted average of this

\footnotetext{
${ }^{9}$ This claimed complexity assumes $W, H$ are initialized as dense matrices. We have assumed this because, if instead they are initialized as sparse matrices, the resulting estimate $W H$ could be far from $\Pi_{n}$. This latter claim follows from the update rule (27): entries of $W, H$ initialized to zero will remain zero; hence, if $W(i,:)$ or $H(:, j)$ contain mostly zeros but $\Pi_{n}(i, j)$ is large, the estimate $W(i,:) H(:, j)$ could be far from $\Pi_{n}(i, j)$. In other words, initializing $W, H$ as sparse matrices requires a sparsity pattern that depends on $\Pi_{n}$, which is unknown at initialization.
} 
aggregated vector and $e_{v}^{\top}$ (Line 5). In essence, we have chosen the learned parameters for Algorithm 7 , rather than learning them. The particular choices guarantee

$$
\left[\begin{array}{c}
h_{1}^{i} \\
\vdots \\
h_{n}^{i}
\end{array}\right]=\alpha_{n} \sum_{j=0}^{i}\left(1-\alpha_{n}\right)^{j} P^{j} \underset{i \rightarrow \infty}{\longrightarrow} \Pi_{n},
$$

where $h_{v}^{i}$ is the representation of $v$ after $i$ iterations of Algorithm 8 , the equality holds by Claim 1 below, and the limit holds by (4). Hence, with these chosen parameters, running Algorithm 7 is effectively the same as computing the power iteration in (28). However, as discussed in Section 5.1, Approx-PageRank and Approx-Contributions are refined versions of this power iteration (with stronger complexity and accuracy guarantees), and these methods have complexity $O\left(n^{2} \log n\right)$. Hence, we strongly suspect that this immediate adaptation of Algorithm 7 will have worse performance than our algorithm (which has complexity $O\left(n^{\bar{c}}\right), \bar{c}<2$ ).

Of course, the preceding paragraph only considers one choice of parameters for Algorithm 7. We could also consider learning these parameters. However, [30] states that running Algorithm 7 with learned parameters causes prohibitively long runtime when $I>2$ (where $I$ is the number of iterations for the algorithm). Hence, for feasible choices of $I$, each node's ultimate representation only depends on its two-step neighborhood. We believe this would lead to very poor accuracy in our setting. This is because, as described in Section 4.2, the set of nodes with large PPR grows with $n$ when $\alpha_{n} \propto 1 / \log n$. Hence, approximating a node's PPR vector while only accounting for its two-step neighborhood will give exceedingly poor accuracy as $n$ grows.
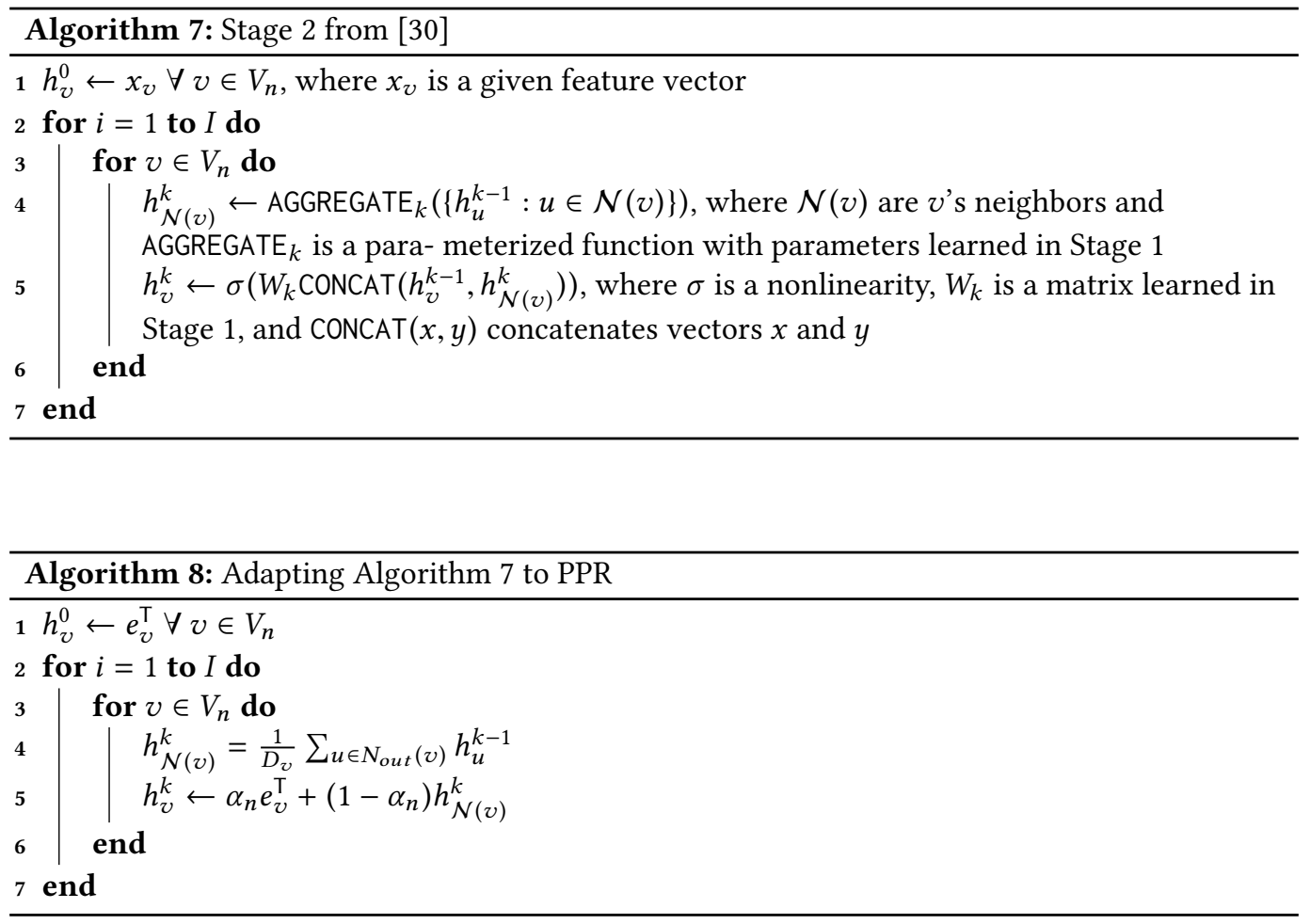
Claim 1. For any $v \in V_{n}$ and any iteration $i$ in Algorithm 8,

$$
h_{v}^{i}=\alpha_{n} e_{v}^{T} \sum_{j=0}^{i}\left(1-\alpha_{n}\right)^{j} P^{j} .
$$

Proof. We use induction. For $i=0$, the claim is immediate by Line 1 of Algorithm 8 . Assuming true for $i-1$, we have

$$
\begin{aligned}
h_{v}^{i} & =\alpha_{n} e_{v}^{\top}+\frac{\left(1-\alpha_{n}\right)}{D_{v}} \sum_{u \in N_{\text {out }}(v)} h_{u}^{k-1} \\
& =\alpha_{n} e_{v}^{\top}+\frac{\left(1-\alpha_{n}\right)}{D_{v}} \sum_{u \in N_{\text {out }}(v)}\left(\alpha_{n} e_{u}^{\top} \sum_{j=0}^{i-1}\left(1-\alpha_{n}\right)^{j} P^{j}\right) \\
& =\alpha_{n} e_{v}^{\top}+\alpha_{n}\left(1-\alpha_{n}\right)\left(\frac{1}{D_{v}} \sum_{u \in N_{\text {out }}(v)} e_{u}^{\mathrm{T}}\right) \sum_{j=0}^{i-1}\left(1-\alpha_{n}\right)^{j} P^{j} \\
& =\alpha_{n} e_{v}^{\top}+\alpha_{n}\left(1-\alpha_{n}\right)\left(e_{v}^{\top} P\right) \sum_{j=0}^{i-1}\left(1-\alpha_{n}\right)^{j} P^{j} \\
& =\alpha_{n} e_{v}^{\top}+\alpha_{n} e_{v}^{\top} \sum_{j=1}^{i}\left(1-\alpha_{n}\right)^{j} P^{j}=\alpha_{n} e_{v}^{T} \sum_{j=0}^{i}\left(1-\alpha_{n}\right)^{j} P^{j},
\end{aligned}
$$

where the first equality holds by Lines 4 and 5 of Algorithm 8, the second uses the inductive hypothesis, and the fourth holds since $P$ is the row-normalized adjacency matrix.

\section{LEMMA 1 PROOF DETAILS}

\section{D.1 Proof of Lemma 2}

The lemma relates the stationary distributions of several Markov chains: those with transition matrices $P_{s}, \tilde{P}_{s}$, and $P_{k}, k \in K_{n}$, where $P_{s}$ and $P_{k}$ are defined in Definition 1 and $\tilde{P}_{s}$ is defined in (6). We will denote these chains by $\left\{X_{i}^{s}\right\}_{i=0}^{\infty},\left\{\tilde{X}_{i}^{s}\right\}_{i=0}^{\infty}$, and $\left\{X_{i}^{k}\right\}_{i=0}^{\infty}, k \in K_{n}$, respectively, in this proof. Our basic approach will be to relate the stationary distributions indirectly via a renewal-reward interpretation of PPR. Hence, we begin by defining this interpretation in Appendix D.1.1. We then prove the lemma in Appendix D.1.2. Recall from the main text that $\mathbb{P}_{G_{n}}[\cdot]$ and $\mathbb{E}_{G_{n}}[\cdot]$ denote probability and expectation with the DCM fixed (as in the statement of the lemma).

D.1.1 Renewal-reward interpretation of PPR. From the dynamics of $\left\{X_{i}^{s}\right\}_{i=0}^{\infty}$ described in Section 2.2 , we can view the time instances of jumps to $s$ as forming a Bernoulli process with parameter $\alpha_{n}$, independent of the random walk. Furthermore, for each $v \in V_{n}$, we can define a reward function $1\left(X_{i}^{s}=v\right)$. Then, letting $L_{s}$ denote the time of the first jump to $s$, we define

$$
\tau_{s}(v)=\sum_{i=0}^{L_{s}-1} 1\left(X_{i}^{s}=v\right)
$$

which, when $X_{0}^{s}=s$, gives the accumulated reward during the first inter-renewal interval. From the renewal-reward theorem (see, for example, Section 5.4 of [26]), it follows that

$$
\lim _{t \rightarrow \infty} \frac{1}{t} \sum_{i=0}^{t-1} 1\left(X_{i}^{s}=v\right)=\alpha_{n} \mathbb{E}_{G_{n}}\left[\tau_{s}(v) \mid X_{0}^{s}=s\right],
$$


where we have also used the fact that $L_{s} \sim$ geometric $\left(\alpha_{n}\right)$. On the other hand, assuming $P_{s}$ is irreducible (which we will return to argue is without loss of generality), we have

$$
\pi_{s}(v)=\lim _{t \rightarrow \infty} \frac{1}{t} \sum_{i=0}^{t-1} 1\left(X_{i}^{s}=v\right) .
$$

Hence, combining (30) and (31) yields

$$
\pi_{s}(v)=\alpha_{n} \mathbb{E}_{G_{n}}\left[\tau_{s}(v) \mid X_{0}^{s}=s\right] \forall v \in V_{n} .
$$

Similarly, for $k \in K_{n}$, we have $\pi_{k}(v)=\alpha_{n} \mathbb{E}_{G_{n}}\left[\tau_{k}(v) \mid X_{0}^{k}=k\right]$, where $\tau_{k}(v)$ is defined as in (29).

For the chain $\left\{\tilde{X}_{i}^{s}\right\}_{i=0}^{\infty}$, we have a similar (though slightly more subtle) renewal-reward interpretation. In particular, recall the dynamics of this chain are as follows: from $v \in V_{n} \backslash K_{n}$, follow the random walk with probability $1-\alpha_{n}$ and jump to $s$ with probability $\alpha_{n}$; from $k \in K_{n}$, jump to $s$ with probability 1 . Hence, while the time instances of jumps to $s$ do not form a Bernoulli process on this chain, they still form a renewal process: inter-renewal intervals are independent (due to the Markov property) and identically-distributed (due to the time invariance of the Markov chain). Additionally, assuming $\tilde{X}_{0}^{s}=s$, the first renewal occurs at $\min \left\{\tilde{L}_{s}, \tilde{H}+1\right\}$, where $\tilde{L}_{s} \sim$ geometric $\left(\alpha_{n}\right)$ and $\tilde{H}=\inf \left\{i \in \mathbb{Z}_{+}: \tilde{X}_{i}^{s} \in K_{n}\right\}$ is the hitting time of $K_{n}$. It follows that

$$
\tilde{\pi}_{s}(v)=\frac{\mathbb{E}_{G_{n}}\left[\tilde{\tau}_{s}(v) \mid \tilde{X}_{0}^{s}=s\right]}{\mathbb{E}_{G_{n}}\left[\min \left\{\tilde{L}_{s}, \tilde{H}+1\right\} \mid \tilde{X}_{0}^{s}=s\right]} \forall v \in V_{n},
$$

where $\tilde{\tau}_{s}(v)=\sum_{i=0}^{\min \left\{\tilde{L}_{s}-1, \tilde{H}\right\}} 1\left(\tilde{X}_{i}^{s}=v\right)$.

Before proceeding, we argue the assumption of irreducibility is without loss of generality for the Markov chains at hand. Consider, for example, $\left\{X_{i}^{s}\right\}_{i=0}^{\infty}$. If this chain is not irreducible, we can define $V_{n, s} \subset V_{n}$ as the states for which a path of positive probability from $s$ to $v$ exists. Then the Markov chain restricted to states $V_{n, s}$ is irreducible: for any $v, w \in V_{n, s}$, we can jump from $v$ to $s$ and then reach $w$ from $s$. We can then compute the stationary distribution $\left\{\pi_{s}(v)\right\}_{v \in V_{n, s}}$ for this irreducible chain and set $\pi_{s}(v)=0 \forall v \in V_{n} \backslash V_{n, s}$ (intuitively, $v$ is unimportant to $s$ if $s$ cannot reach $v$, so its PPR should be zero). Note this is consistent with the derivation above. In particular, (30) and (31) hold for the chain restricted to states $V_{n, s}$, so (32) holds for $v \in V_{n, s}$; on the other hand, both sides of (32) are zero for $v \notin V_{n, s}$.

D.1.2 Proof of the lemma. Equipped with this renewal-reward interpretation, we will relate $\pi_{s}, \tilde{\pi}_{s}$, and $\pi_{k}, k \in K_{n}$ by relating $\mathbb{E}_{G_{n}}\left[\tau_{s}(v) \mid X_{0}^{s}=s\right], \mathbb{E}_{G_{n}}\left[\tilde{\tau}_{s}(v) \mid \tilde{X}_{0}^{s}=s\right]$, and $\mathbb{E}_{G_{n}}\left[\tau_{k}(v) \mid X_{0}^{k}=k\right], k \in K_{n}$. For this, we define $H=\inf \left\{i \in \mathbb{Z}_{+}: X_{i}^{s} \in K_{n}\right\}$, the quantity analogous to $\tilde{H}$ instead defined on $\left\{X_{i}^{s}\right\}_{i=0}^{\infty}$.

Because the dynamics of $\left\{X_{i}^{s}\right\}_{i=0}^{\infty}$ and $\left\{\tilde{X}_{i}^{s}\right\}_{i=0}^{\infty}$ only differ when $K_{n}$ is reached, we can immediately obtain several relationship between the quantities computed on these chains. In particular, if $K_{n}$ is not reached before the first renewal (i.e. if $L_{s} \leq H, \tilde{L}_{s} \leq \tilde{H}$ ), the chains have identical dynamics. Therefore, we have $\forall v \in V_{n}$,

$$
\mathbb{E}_{G_{n}}\left[\tau_{s}(v) \mid L_{s} \leq H, X_{0}^{s}=s\right]=\mathbb{E}_{G_{n}}\left[\tilde{\tau}_{s}(v) \mid \tilde{L}_{s} \leq \tilde{H}, \tilde{X}_{0}^{s}=s\right] .
$$

Furthermore, $\tilde{\tau}_{s}(v)=0$ when $v \in K_{n}$ and $\tilde{L}_{s} \leq \tilde{H}$ (i.e. when $K_{n}$ is not reached before the first renewal), so we may rewrite this as

$$
\mathbb{E}_{G_{n}}\left[\tau_{s}(v) \mid L_{s} \leq H, X_{0}^{s}=s\right]=U_{v} \mathbb{E}_{G_{n}}\left[\tilde{\tau}_{s}(v) \mid \tilde{L}_{s} \leq \tilde{H}, \tilde{X}_{0}^{s}=s\right]
$$


By a similar argument, if $K_{n}$ is reached before the first renewal $\left(L_{s}>H, \tilde{L}_{s}>\tilde{H}\right)$, the dynamics of the chains differ after $H, \tilde{H}$, but they remain the same up to and including $H, \tilde{H}$. Hence, $\forall k \in K_{n}$,

$$
\mathbb{P}_{G_{n}}\left[X_{H}^{s}=k, L_{s}>H \mid X_{0}^{s}=s\right]=\mathbb{P}_{G_{n}}\left[\tilde{X}_{\tilde{H}}^{s}=k, \tilde{L}_{s}>\tilde{H} \mid \tilde{X}_{0}^{s}=s\right],
$$

which also implies

$$
\mathbb{P}_{G_{n}}\left[L_{s} \leq H \mid X_{0}^{s}=s\right]=\mathbb{P}_{G_{n}}\left[\tilde{L}_{s} \leq \tilde{H} \mid \tilde{X}_{0}^{s}=s\right] .
$$

We can obtain another expression for the right side of (34). In particular, since jumps from $k$ to $s$ occur with probability 1 on the $\left\{\tilde{X}_{i}^{s}\right\}_{i=0}^{\infty}$ chain, $k$ is visited at most one time before the first renewal, i.e. $\tilde{\tau}_{s}(k) \in\{0,1\}$. Furthermore, $\tilde{\tau}_{s}(k)=1$ if and only if $\tilde{L}_{s}>\tilde{H}$ and $\tilde{X}_{\tilde{H}}^{s}=k$. Hence,

$$
\mathbb{P}_{G_{n}}\left[X_{H}^{s}=k, L_{s}>H \mid X_{0}^{s}=s\right]=\mathbb{E}_{G_{n}}\left[\tilde{\tau}_{s}(k) \mid \tilde{X}_{0}^{s}=s\right] \forall k \in K_{n} .
$$

If instead $K_{n}$ is reached, the dynamics of $\left\{X_{i}^{s}\right\}_{i=0}^{\infty}$ and $\left\{\tilde{X}_{i}^{s}\right\}_{i=0}^{\infty}$ differ. In this case, we claim

$$
\begin{gathered}
\mathbb{E}_{G_{n}}\left[\tau_{s}(v) \mid X_{H}^{s}=k, L_{s}>H, X_{0}^{s}=s\right]=U_{v} \mathbb{E}_{G_{n}}\left[\tilde{\tau}_{s}(v) \mid \tilde{X}_{\tilde{H}}^{s}=k, \tilde{L}_{s}>\tilde{H}, \tilde{X}_{0}^{s}=s\right] \\
+\mathbb{E}_{G_{n}}\left[\tau_{k}(v) \mid X_{0}^{k}=k\right],
\end{gathered}
$$

which we will return to prove shortly. (In essence, (37) counts the number visits to $v$ before and after reaching $k$ using the $\left\{\tilde{X}_{i}^{s}\right\}_{i=0}^{\infty}$ and $\left\{X_{i}^{k}\right\}_{i=1}^{k}$ chains, respectively.)

By (33), (34), (35), (36), and (37), and the law of total expectation,

$$
\mathbb{E}_{G_{n}}\left[\tau_{s}(v) \mid X_{0}^{s}=s\right]=U_{v} \mathbb{E}_{G_{n}}\left[\tilde{\tau}_{s}(v) \mid \tilde{X}_{0}^{s}=s\right]+\sum_{k \in K_{n}} \mathbb{E}_{G_{n}}\left[\tilde{\tau}_{s}(k) \mid \tilde{X}_{0}^{s}=s\right] \mathbb{E}_{G_{n}}\left[\tau_{k}(v) \mid X_{0}^{k}=k\right] .
$$

We then use the renewal-reward interpretation from Appendix D.1.1 to translate this equation back to stationary distributions. Specifically, multiplying by $\alpha_{n}$ on both sides, and multiplying and dividing by $\mathbb{E}_{G_{n}}\left[\min \left\{\tilde{L}_{s}, \tilde{H}+1\right\} \mid \tilde{X}_{0}^{s}=s\right]$ on the right side, gives

$$
\pi_{s}(v)=\mathbb{E}_{G_{n}}\left[\min \left\{\tilde{L}_{s}, \tilde{H}+1\right\} \mid \tilde{X}_{0}^{s}=s\right]\left(\alpha_{n} U_{v} \tilde{\pi}_{s}(v)+\sum_{k \in K_{n}} \tilde{\pi}_{s}(k) \pi_{k}(v)\right) .
$$

Then, summing over $v \in V_{n}$ (assuming stationary distributions are normalized to sum to 1),

$$
\begin{aligned}
1 & =\mathbb{E}_{G_{n}}\left[\min \left\{\tilde{L}_{s}, \tilde{H}+1\right\} \mid \tilde{X}_{0}^{s}=s\right]\left(\alpha_{n} \tilde{\pi}_{s}\left(V_{n} \backslash K_{n}\right)+\tilde{\pi}_{s}\left(K_{n}\right)\right) \\
\Rightarrow & \mathbb{E}_{G_{n}}\left[\min \left\{\tilde{L}_{s}, \tilde{H}+1\right\} \mid \tilde{X}_{0}^{s}=s\right]=\frac{1}{\alpha_{n}+\left(1-\alpha_{n}\right) \tilde{\pi}_{s}\left(K_{n}\right)} .
\end{aligned}
$$

Finally, combining (38) and (39) completes the proof.

We now return to prove (37). First, by definition of $\tau_{s}(v)$,

$$
\begin{aligned}
\mathbb{E}_{G_{n}}\left[\tau_{s}(v) \mid X_{H}^{s}=k, L_{s}>H, X_{0}^{s}=s\right]=\mathbb{E}_{G_{n}} & {\left[\sum_{i=0}^{H-1} 1\left(X_{i}^{s}=v\right) \mid X_{H}^{s}=k, L_{s}>H, X_{0}^{s}=s\right] } \\
+ & \mathbb{E}_{G_{n}}\left[\sum_{i=H}^{L_{s}-1} 1\left(X_{i}^{s}=v\right) \mid X_{H}^{s}=k, L_{s}>H, X_{0}^{s}=s\right] .
\end{aligned}
$$

Now consider the first summand in (40). By the preceding arguments, $\left\{X_{i}^{s}\right\}_{i=0}^{\infty},\left\{\tilde{X}_{i}^{s}\right\}_{i=0}^{\infty}$ have the same dynamics before $H, \tilde{H}$, so

$$
\mathbb{E}_{G_{n}}\left[\sum_{i=0}^{H-1} 1\left(X_{i}^{s}=v\right) \mid X_{H}^{s}=k, L_{s}>H, X_{0}^{s}=s\right]=\mathbb{E}_{G_{n}}\left[\sum_{i=0}^{\tilde{H}-1} 1\left(\tilde{X}_{i}^{s}=v\right) \mid \tilde{X}_{\tilde{H}}^{s}=k, \tilde{L}_{s}>\tilde{H}, \tilde{X}_{0}^{s}=s\right] .
$$


For $v \in V_{n} \backslash K_{n}$ (i.e. $U_{v}=1$ ), we can write

$$
\begin{aligned}
\mathbb{E}_{G_{n}} & {\left[\sum_{i=0}^{\tilde{H}-1} 1\left(\tilde{X}_{i}^{s}=v\right) \mid \tilde{X}_{\tilde{H}}^{s}=k, \tilde{L}_{s}>\tilde{H}, \tilde{X}_{0}^{s}=s\right]=\mathbb{E}_{G_{n}}\left[\sum_{i=0}^{\tilde{H}} 1\left(\tilde{X}_{i}^{s}=v\right) \mid \tilde{X}_{\tilde{H}}^{s}=k, \tilde{L}_{s}>\tilde{H}, \tilde{X}_{0}^{s}=s\right] } \\
= & \mathbb{E}_{G_{n}}\left[\sum_{i=0}^{\min \left\{\tilde{L}_{s}-1, \tilde{H}\right\}} 1\left(\tilde{X}_{i}^{s}=v\right) \mid \tilde{X}_{\tilde{H}}^{s}=k, \tilde{L}_{s}>\tilde{H}, \tilde{X}_{0}^{s}=s\right]=\mathbb{E}_{G_{n}}\left[\tilde{\tau}_{s}(v) \mid \tilde{X}_{\tilde{H}}^{s}=k, \tilde{L}_{s}>\tilde{H}, \tilde{X}_{0}^{s}=s\right],
\end{aligned}
$$

where the first equality holds since $v \in V_{n} \backslash K_{n}$ and by conditioning on $\left\{\tilde{X}_{\tilde{H}}^{s}=k\right\}\left(k \in K_{n}\right)$, the second holds by conditioning on $\left\{\tilde{L}_{s}>\tilde{H}\right\}$, and the third holds by definition of $\tilde{\tau}_{s}(v)$. Note that if $v \in K_{n}$ (i.e. $U_{v}=0$ ), we simply have

$$
\mathbb{E}_{G_{n}}\left[\sum_{i=0}^{\tilde{H}-1} 1\left(\tilde{X}_{i}^{s}=v\right) \mid \tilde{X}_{\tilde{H}}^{s}=k, \tilde{L}_{s}>\tilde{H}, \tilde{X}_{0}^{s}=s\right]=0,
$$

which holds by definition of $\tilde{H}$. To summarize, we have shown

$$
\mathbb{E}_{G_{n}}\left[\sum_{i=0}^{H-1} 1\left(X_{i}^{s}=v\right) \mid X_{H}^{s}=k, L_{s}>H, X_{0}^{s}=s\right]=U_{v} \mathbb{E}_{G_{n}}\left[\tilde{\tau}_{s}(v) \mid \tilde{X}_{\tilde{H}}^{s}=k, \tilde{L}_{s}>\tilde{H}, \tilde{X}_{0}^{s}=s\right] .
$$

Next, consider the second summand in (40). We rewrite this term as

$$
\frac{\mathbb{E}_{G_{n}}\left[\sum_{i=H}^{L_{s}-1} 1\left(X_{i}^{s}=v, X_{H}^{s}=k, X_{0}^{s}=s\right) 1\left(L_{s}>H\right)\right]}{\mathbb{P}_{G_{n}}\left[X_{H}^{s}=k, L_{s}>H, X_{0}^{s}=s\right]},
$$

and we focus on the numerator. First, we note $1\left(L_{s}>H\right)=\sum_{l>h} 1\left(L_{s}=l, H=h\right)$, where the sum is taken over $\left\{(l, h) \in \mathbb{Z}_{+} \times \mathbb{Z}_{+}: l>h\right\}$. Substituting and using linearity gives

$$
\begin{aligned}
\sum_{l>h} \mathbb{E}_{G_{n}}\left[\sum_{i=H}^{L_{s}-1} 1\left(X_{i}^{s}=v, X_{H}^{s}=k, X_{0}^{s}=s\right) 1\left(L_{s}=l, H=h\right)\right] \\
=\sum_{l>h} \mathbb{E}_{G_{n}}\left[\sum_{i=h}^{l-1} 1\left(X_{i}^{s}=v, X_{h}^{s}=k, X_{0}^{s}=s\right) 1\left(L_{s}=l, H=h\right)\right] \\
=\sum_{l>h} \sum_{i=h}^{l-1} \mathbb{P}_{G_{n}}\left[X_{i}^{s}=v, X_{h}^{s}=k, X_{0}^{s}=s, L_{s}=l, H=h\right]
\end{aligned}
$$

Rewriting the summand in (43) as

$$
\mathbb{P}_{G_{n}}\left[X_{i}^{s}=v, X_{0}^{s}=s, L_{s}=l, H=h \mid X_{h}^{s}=k\right] \mathbb{P}_{G_{n}}\left[X_{h}^{s}=k\right],
$$

we next aim to apply the Markov property to the conditional probability above. For this, we write $\left\{L_{s}=l\right\}=A_{s, l} \cap\left(\cap_{j=0}^{l-1} A_{s, j}^{C}\right)$, where $A_{s, j}$ denotes the event that a jump to $s$ occurs at step $j$ of the random walk. We then have

$$
\left\{X_{i}^{s}=v, X_{0}^{s}=s, L_{s}=l, H=h\right\}=\left\{X_{i}^{s}=v, A_{s, l}, \cap_{j=h+1}^{l-1} A_{s, j}^{C}\right\} \cap\left\{H=h, \cap_{j=0}^{h} A_{s, j}^{C}, X_{0}^{s}=s\right\}
$$


where on the right side, the first event is the future and the second event is the past, when $h$ is viewed as the present. Hence, the Markov property implies

$$
\begin{aligned}
\mathbb{P}_{G_{n}}\left[X_{i}^{s}=v, X_{0}^{s}=s, L_{s}=l, H=h \mid X_{h}^{s}=k\right]= & \mathbb{P}_{G_{n}}\left[X_{i}^{s}=v, A_{s, l}, \cap_{j=h}^{l-1} A_{s, j}^{C} \mid X_{h}^{s}=k\right] \\
& \times \mathbb{P}_{G_{n}}\left[H=h, \cap_{j=0}^{h-1} A_{s, j}^{C}, X_{0}^{s}=s \mid X_{h}^{s}=k\right] .
\end{aligned}
$$

Furthermore, by the time invariance of the Markov chain,

$$
\begin{aligned}
\mathbb{P}_{G_{n}}\left[X_{i}^{s}=v, A_{s, l}, \cap_{j=h}^{l-1} A_{s, j}^{C} \mid X_{h}^{s}=k\right] & =\mathbb{P}_{G_{n}}\left[X_{i-h}^{s}=v, A_{s, l-h}, \cap_{j=0}^{l-h-1} A_{s, j}^{C} \mid X_{0}^{s}=k\right] \\
& =\mathbb{P}_{G_{n}}\left[X_{i-h}^{s}=v, L_{s}=l-h \mid X_{0}^{s}=k\right] .
\end{aligned}
$$

Finally, by definition of $A_{s, j}$, we have

$$
\mathbb{P}_{G_{n}}\left[H=h, \cap_{j=0}^{h-1} A_{s, j}^{C}, X_{0}^{s}=s \mid X_{h}^{s}=k\right]=\mathbb{P}_{G_{n}}\left[H=h, L_{s}>h, X_{0}^{s}=s \mid X_{h}^{s}=k\right] .
$$

Combining (43), (44), (45), and (46) then yields

$$
\begin{aligned}
\sum_{l>h} & \mathbb{E}_{G_{n}}\left[\sum_{i=H}^{L_{s}-1} 1\left(X_{i}^{s}=v, X_{H}^{s}=k, X_{0}^{s}=s\right) 1\left(L_{s}=l, H=h\right)\right] \\
= & \sum_{l>h} \sum_{i=h}^{l-1} \mathbb{P}_{G_{n}}\left[X_{i-h}^{s}=v, L_{s}=l-h \mid X_{0}^{s}=k\right] \mathbb{P}_{G_{n}}\left[H=h, L_{s}>h, X_{0}^{s}=s, X_{h}^{s}=k\right] \\
= & \sum_{h \in \mathbb{Z}_{+}} \mathbb{P}_{G_{n}}\left[H=h, L_{s}>h, X_{0}^{s}=s, X_{h}^{s}=k\right] \sum_{l=h+1}^{\infty} \sum_{i=0}^{l-h-1} \mathbb{P}_{G_{n}}\left[X_{i}^{s}=v, L_{s}=l-h \mid X_{0}^{s}=k\right],
\end{aligned}
$$

where in the second equality we have simply rearranged terms and rewritten indices. For the inner double summation, we have

$$
\begin{aligned}
& \sum_{l=h+1}^{\infty} \sum_{i=0}^{l-h-1} \mathbb{P}_{G_{n}}\left[X_{i}^{s}=v, L_{s}=l-h \mid X_{0}^{s}=k\right] \\
& =\frac{\sum_{l=h+1}^{\infty} \mathbb{E}_{G_{n}}\left[\sum_{i=0}^{l-h-1} 1\left(X_{i}^{s}=v\right) 1\left(L_{s}=l-h, X_{0}^{s}=k\right)\right]}{\mathbb{P}_{G_{n}}\left[X_{0}^{s}=k\right]} \\
& =\frac{\sum_{l=h+1}^{\infty} \mathbb{E}_{G_{n}}\left[\sum_{i=0}^{L_{s}-1} 1\left(X_{i}^{s}=v\right) 1\left(L_{s}=l-h, X_{0}^{s}=k\right)\right]}{\mathbb{P}_{G_{n}}\left[X_{0}^{s}=k\right]} \\
& =\frac{\mathbb{E}_{G_{n}}\left[\sum_{i=0}^{L_{s}-1} 1\left(X_{i}^{s}=v\right) 1\left(X_{0}^{s}=k\right) \sum_{l=h+1}^{\infty} 1\left(L_{s}=l-h\right)\right]}{\mathbb{P}_{G_{n}}\left[X_{0}^{s}=k\right]} \\
& =\mathbb{E}_{G_{n}}\left[\sum_{i=0}^{L_{s}-1} 1\left(X_{i}^{s}=v\right) \mid X_{0}^{s}=k\right]=\mathbb{E}_{G_{n}}\left[\tau_{s}(v) \mid X_{0}^{s}=k\right]=\mathbb{E}_{G_{n}}\left[\tau_{k}(v) \mid X_{0}^{k}=k\right],
\end{aligned}
$$

where the first three steps are straightforward, the fourth step uses the fact that $L_{s}$ is integer-valued and $a$.s. finite, and the fifth step follows by definition. The final inequality follows because $\tau_{s}(v)$ and $\tau_{k}(v)$ count the number of visits to $v$ on the $\left\{X_{i}^{s}\right\}_{i=0}^{\infty}$ and $\left\{X_{i}^{k}\right\}_{i=0}^{\infty}$ chains before jumps occur, and before jumps occur, these chains have the same dynamics (since they only differ in jump locations, 
$s$ versus $k$ ). Substituting into (47) gives

$$
\begin{aligned}
& \sum_{l>h} \mathbb{E}_{G_{n}}\left[\sum_{i=H}^{L_{s}-1} 1\left(X_{i}^{s}=v, X_{H}^{s}=k, X_{0}^{s}=s\right) 1\left(L_{s}=l, H=h\right)\right] \\
& =\mathbb{E}_{G_{n}}\left[\tau_{k}(v) \mid X_{0}^{k}=k\right] \sum_{h \in \mathbb{Z}_{+}} \mathbb{P}_{G_{n}}\left[H=h, L_{s}>h, X_{0}^{s}=s, X_{h}^{s}=k\right] \\
& =\mathbb{E}_{G_{n}}\left[\tau_{k}(v) \mid X_{0}^{k}=k\right] \mathbb{P}_{G_{n}}\left[L_{s}>H, X_{0}^{s}=s, X_{H}^{s}=k\right] .
\end{aligned}
$$

Hence, combining (42) and (48) yields

$$
\mathbb{E}_{G_{n}}\left[\sum_{i=H}^{L_{s}-1} 1\left(X_{i}^{s}=v\right) \mid X_{H}^{s}=k, L_{s}>H, X_{0}^{s}=s\right]=\mathbb{E}_{G_{n}}\left[\tau_{k}(v) \mid X_{0}^{k}=k\right] .
$$

Finally, (40), (41), and (49) complete the proof of (37).

\section{D.2 Proof of Lemma 3}

We aim to bound $\left\|\pi_{s}-\left(\alpha_{n} e_{s}^{\top}+\sum_{k \in K_{n}} \beta_{s}(k) \pi_{k}\right)\right\|_{1}$, where

$$
\beta_{s}(k)=\frac{\tilde{\pi}_{s}(k)}{\alpha_{n}+\left(1-\alpha_{n}\right) \tilde{\pi}_{s}\left(K_{n}\right)} \forall k \in K_{n} .
$$

Using Lemma 2, we can write

$$
\left\|\pi_{s}-\left(\alpha_{n} e_{s}^{\mathrm{T}}+\sum_{k \in K_{n}} \beta_{s}(k) \pi_{k}\right)\right\|_{1}=\sum_{v \in V_{n}}\left|\frac{\alpha_{n} U_{v} \tilde{\pi}_{s}(v)}{\alpha_{n}+\left(1-\alpha_{n}\right) \tilde{\pi}_{s}\left(K_{n}\right)}-\alpha_{n} 1(v=s)\right| .
$$

We next claim that the terms in the absolute values are nonnegative. This is obvious for $v \neq s$. For $v=s$, since $U_{s}=1$, we aim to show

$$
\tilde{\pi}_{s}(s) \geq \alpha_{n}+\left(1-\alpha_{n}\right) \tilde{\pi}_{s}\left(K_{n}\right) .
$$

To this end, first note that by $\tilde{\pi}_{s}=\tilde{\pi}_{s} \tilde{P}_{s}$ and $\tilde{\pi}_{s} 1_{n}=1$,

$$
\tilde{\pi}_{s}=\left(1-\alpha_{n}\right) \tilde{\pi}_{s}\left(\tilde{P}+e_{K_{n}} e_{s}^{\top}\right)+\alpha_{n} e_{s}^{\top},
$$

which implies

$$
\tilde{\pi}_{s}=\alpha_{n} e_{s}^{\top}\left(I-\left(1-\alpha_{n}\right)\left(\tilde{P}+e_{K_{n}} e_{s}^{\top}\right)\right)^{-1}=\alpha_{n} e_{s}^{\top} \sum_{i=0}^{\infty}\left(1-\alpha_{n}\right)^{i}\left(\tilde{P}+e_{K_{n}} e_{s}^{\top}\right)^{i} .
$$

Using (51), we have

$$
\begin{aligned}
\tilde{\pi}_{s}(s)= & \alpha_{n} e_{s}^{\mathrm{T}} \sum_{i=0}^{\infty}\left(1-\alpha_{n}\right)^{i}\left(\tilde{P}+e_{K_{n}} e_{s}^{\top}\right)^{i} e_{s}=\alpha_{n}+\alpha_{n} e_{s}^{\mathrm{T}} \sum_{i=1}^{\infty}\left(1-\alpha_{n}\right)^{i}\left(\tilde{P}+e_{K_{n}} e_{s}^{\mathrm{T}}\right)^{i} e_{s} \\
= & \alpha_{n}+\alpha_{n}\left(1-\alpha_{n}\right) e_{s}^{\top} \sum_{i=0}^{\infty}\left(1-\alpha_{n}\right)^{i}\left(\tilde{P}+e_{K_{n}} e_{s}^{\mathrm{T}}\right)^{i} e_{K_{n}} e_{s}^{\top} e_{s} \\
& +\alpha_{n}\left(1-\alpha_{n}\right) e_{s}^{\top} \sum_{i=0}^{\infty}\left(1-\alpha_{n}\right)^{i}\left(\tilde{P}+e_{K_{n}} e_{s}^{\top}\right)^{i} \tilde{P} e_{s}^{\top},
\end{aligned}
$$

and so, dropping a nonnegative term, we obtain

$$
\tilde{\pi}_{s}(s) \geq \alpha_{n}+\alpha_{n}\left(1-\alpha_{n}\right) e_{s}^{\top} \sum_{i=0}^{\infty}\left(1-\alpha_{n}\right)^{i}\left(\tilde{P}+e_{K_{n}} e_{s}^{\top}\right)^{i} e_{K_{n}} e_{s}^{\top} e_{s}=\alpha_{n}+\left(1-\alpha_{n}\right) \tilde{\pi}_{s} e_{K_{n}} .
$$


This establishes (50), since $\tilde{\pi}_{s} e_{K_{n}}=\tilde{\pi}_{s}\left(K_{n}\right)$. Hence, we have shown

$$
\left\|\pi_{s}-\left(\alpha_{n} e_{s}^{\mathrm{T}}+\sum_{k \in K_{n}} \beta_{s}(k) \pi_{k}\right)\right\|_{1}=\alpha_{n}\left(\frac{\tilde{\pi}_{s}\left(V_{n} \backslash K_{n}\right)}{\alpha_{n}+\left(1-\alpha_{n}\right) \tilde{\pi}_{s}\left(K_{n}\right)}-1\right) .
$$

(We pause to note that since $\tilde{\pi}_{s}\left(V_{n} \backslash K_{n}\right) \leq 1$ and $\tilde{\pi}_{s}\left(K_{n}\right) \geq 0$,

$$
\left\|\pi_{s}-\left(\alpha_{n} e_{s}^{\top}+\sum_{k \in K_{n}} \beta_{s}(k) \pi_{k}\right)\right\|_{1} \leq \alpha_{n}\left(\frac{1}{\alpha_{n}}-1\right)=1-\alpha_{n},
$$

i.e. $\pi_{s}$ is at $l_{1}$ distance at most $1-\alpha_{n}$ from a linear combination of $e_{s}^{\top}$ and $\left\{\pi_{k}\right\}_{k \in K_{n}}$.) We next bound the right side of (52) in terms of $\mu_{s}^{(m)}$, as in the statement of the lemma. We begin by establishing a relationship between $\tilde{\pi}_{s}$ and $\mu_{s}$, where

$$
\mu_{s}=\lim _{m \rightarrow \infty} \mu_{s}^{(m)}=e_{s}^{\top} \sum_{i=0}^{\infty}\left(1-\alpha_{n}\right)^{i} \tilde{P}^{i}=e_{s}^{\mathrm{T}}\left(I-\left(1-\alpha_{n}\right) \tilde{P}\right)^{-1} .
$$

To this end, consider the matrix inversion in (51). By the Sherman-Morrison-Woodbury formula (see, for example, Section 6.4 of [34]),

$$
\begin{aligned}
& \left(I-\left(1-\alpha_{n}\right)\left(\tilde{P}+e_{K_{n}} e_{s}^{\top}\right)\right)^{-1}=\left(\left(I-\left(1-\alpha_{n}\right) \tilde{P}\right)-\left(1-\alpha_{n}\right) e_{K_{n}} e_{s}^{\mathrm{T}}\right)^{-1} \\
& \quad=\left(I-\left(1-\alpha_{n}\right) \tilde{P}\right)^{-1}+\frac{\left(I-\left(1-\alpha_{n}\right) \tilde{P}\right)^{-1}\left(1-\alpha_{n}\right) e_{K_{n}} e_{s}^{\top}\left(I-\left(1-\alpha_{n}\right) \tilde{P}\right)^{-1}}{1-e_{s}^{\top}\left(I-\left(1-\alpha_{n}\right) \tilde{P}\right)^{-1}\left(1-\alpha_{n}\right) e_{K_{n}}} .
\end{aligned}
$$

It follows that, for each $v \in V_{n}$,

$$
\begin{aligned}
\tilde{\pi}_{s}(v) & =\alpha_{n} e_{s}^{\mathrm{T}}\left(I-\left(1-\alpha_{n}\right)\left(\tilde{P}+e_{K_{n}} e_{s}^{\mathrm{T}}\right)\right)^{-1} e_{v} \\
& =\alpha_{n} e_{s}^{\mathrm{\top}}\left(I-\left(1-\alpha_{n}\right) \tilde{P}\right)^{-1} e_{v}+\alpha_{n} e_{s}^{\mathrm{\top}} \frac{\left(I-\left(1-\alpha_{n}\right) \tilde{P}\right)^{-1}\left(1-\alpha_{n}\right) e_{K_{n}} e_{s}^{\mathrm{T}}\left(I-\left(1-\alpha_{n}\right) \tilde{P}\right)^{-1}}{1-e_{s}^{\top}\left(I-\left(1-\alpha_{n}\right) \tilde{P}\right)^{-1}\left(1-\alpha_{n}\right) e_{K_{n}}} e_{v} \\
& =\alpha_{n} \mu_{s}(v)\left(1+\frac{\left(1-\alpha_{n}\right) \mu_{s}\left(K_{n}\right)}{1-\left(1-\alpha_{n}\right) \mu_{s}\left(K_{n}\right)}\right)=\frac{\alpha_{n} \mu_{s}(v)}{1-\left(1-\alpha_{n}\right) \mu_{s}\left(K_{n}\right)}
\end{aligned}
$$

where the first three equalities follow from (51), (55), and (54), respectively, and the fourth involves simple manipulations. We can then combine (52) and (56) to obtain

$$
\left\|\pi_{s}-\left(\alpha_{n} e_{s}^{\top}+\sum_{k \in K} \beta_{s}(k) \pi_{k}\right)\right\|_{1}=\alpha_{n}\left(\mu_{s}\left(V_{n} \backslash K_{n}\right)-1\right) .
$$

Next, we observe

$$
\begin{aligned}
\mu_{s}\left(V_{n} \backslash K_{n}\right) & =\mu_{s}^{(m)}\left(V_{n} \backslash K_{n}\right)+e_{s}^{\mathrm{T}} \sum_{i=m+1}^{\infty}\left(1-\alpha_{n}\right)^{i} \tilde{P}^{i} e_{V_{n} \backslash K_{n}} \\
& =\mu_{s}^{(m)}\left(V_{n} \backslash K_{n}\right)+e_{s}^{\mathrm{T}}\left(1-\alpha_{n}\right)^{m} \tilde{P}^{m} \sum_{i=1}^{\infty}\left(1-\alpha_{n}\right)^{i} \tilde{P}^{i} e_{V_{n} \backslash K_{n}} \\
& =\mu_{s}^{(m)}\left(V_{n} \backslash K_{n}\right)+\left(\mu_{s}^{(m)}-\mu_{s}^{(m-1)}\right) \sum_{i=1}^{\infty}\left(1-\alpha_{n}\right)^{i} \tilde{P}^{i} e_{V_{n} \backslash K_{n}},
\end{aligned}
$$


where we have used (19) and (54). We next claim $\tilde{P} e_{V_{n} \backslash K_{n}} \leq e_{V_{n} \backslash K_{n}}$, where the inequality is taken componentwise. To see this, first let $\left(\tilde{P} e_{V_{n} \backslash K_{n}}\right)(i)$ denote the $i$-th component of $\tilde{P} e_{V_{n} \backslash K_{n}}$. Then

$$
\left(\tilde{P} e_{V_{n} \backslash K_{n}}\right)(i)=\sum_{j=1}^{n} \tilde{P}(i, j) e_{V_{n} \backslash K_{n}}(j)=U_{i} \sum_{j=1}^{n} P(i, j) e_{V_{n} \backslash K_{n}}(j) \leq U_{i} \sum_{j=1}^{n} P(i, j)=U_{i}=e_{V_{n} \backslash K_{n}}(i),
$$

where the second equality uses the definition of $\tilde{P}$, the third equality holds because $P$ is row stochastic, and the remaining steps are straightforward. It follows that

$$
\sum_{i=1}^{\infty}\left(1-\alpha_{n}\right)^{i} \tilde{P}^{i} e_{V_{n} \backslash K_{n}} \leq\left(\sum_{i=1}^{\infty}\left(1-\alpha_{n}\right)^{i}\right) e_{V_{n} \backslash K_{n}}=\left(\frac{1-\alpha_{n}}{\alpha_{n}}\right) e_{V_{n} \backslash K_{n}},
$$

where the inequality is componentwise. Combining (58) and (59),

$$
\begin{aligned}
\mu_{s}\left(V_{n} \backslash K_{n}\right) & \leq \mu_{s}^{(m)}\left(V_{n} \backslash K_{n}\right)+\left(\mu_{s}^{(m)}-\mu_{s}^{(m-1)}\right)\left(\frac{1-\alpha_{n}}{\alpha_{n}}\right) e_{V_{n} \backslash K_{n}} \\
& =\frac{1}{\alpha_{n}} e_{s}^{\top}\left(1-\alpha_{n}\right)^{m} \tilde{P}^{m} e_{V_{n} \backslash K_{n}}+\mu_{s}^{(m-1)}\left(V_{n} \backslash K_{n}\right),
\end{aligned}
$$

where we have also used $\mu_{s}^{(m)} \geq \mu_{s}^{(m-1)}$ (componentwise). Finally, (57) and (60) together imply

$$
\left\|\pi_{s}-\left(\alpha_{n} e_{s}^{\top}+\sum_{k \in K} \beta_{s}(k) \pi_{k}\right)\right\|_{1} \leq \alpha_{n}\left(\mu_{s}^{(m-1)}\left(V_{n} \backslash K_{n}\right)-1\right)+e_{s}^{\top}\left(1-\alpha_{n}\right)^{m} \tilde{P}^{m} e_{V_{n} \backslash K_{n}},
$$

which is what we set out to prove.

\section{D.3 Proof of Lemma 4}

We will use Algorithm 9 in Appendix D.6, which simultaneously constructs a graph and a tree. We will let $H_{n}$ and $\hat{H}_{n}$ denote this graph and this tree, respectively. From $H_{n}$, we define

$$
v_{s}^{(m)}=e_{s}^{\mathrm{T}} \sum_{j=0}^{m}\left(1-\alpha_{n}\right)^{j} \tilde{Q}^{j},
$$

where $\tilde{Q}(i, j)=U_{i} Q(i, j)$ and $Q$ is the adjacency matrix of $H_{n}$, normalized to be row stochastic. Note this is simply (19), i.e. the definition as $\mu_{s}^{(m)}$, but computed on $H_{n}$ (while $\mu_{s}^{(m)}$ is computed on $G_{n}$ ). Similarly, using $\hat{H}_{n}$, recursively define

$$
\hat{v}_{\phi}(\phi)=1, \quad \hat{v}_{\phi}((\mathbf{i}, j))=\hat{v}_{\phi}(\mathbf{i}) \frac{\left(1-\alpha_{n}\right) U_{\mathbf{i}}}{D_{\mathbf{i}}},(\mathbf{i}, j) \in \hat{A}_{l}, l>0,
$$

which is (22) but computed on $\hat{H}_{n}$ instead of $\hat{G}_{n}$. With this notation in place, we will show

$$
\begin{aligned}
\mu_{s}^{(m)}\left(V_{n} \backslash K_{n}\right) \mid\left\{\tau_{G}>m, U_{s}=1\right\} & \stackrel{\mathcal{D}}{=} v_{s}^{(m)}\left(V_{n} \backslash K_{n}\right) \mid\left\{\tau_{S}>m\right\}, \\
v_{s}^{(m)}\left(V_{n} \backslash K_{n}\right) & =\sum_{j=0}^{m} \sum_{\mathbf{i} \in \hat{A}_{j}} U_{\mathbf{i}} \hat{v}_{\phi}(\mathbf{i}) \text { when } \tau_{S}>m, \\
\sum_{j=0}^{m} \sum_{\mathbf{i} \in \hat{A}_{j}} U_{\mathbf{i}} \hat{v}_{\phi}(\mathbf{i}) \mid\left\{\tau_{S}>m\right\} & \stackrel{\mathcal{D}}{=} \sum_{j=0}^{m} \sum_{\mathbf{i} \in \hat{A}_{j}} U_{\mathbf{i}} \hat{\mu}_{\phi}(\mathbf{i}),
\end{aligned}
$$

which, taken together, establish the lemma. (We remind the reader that $\tau_{G}$ and $\tau_{S}$, respectively, denote the first iteration at which certain events occur in Algorithm 5 and Algorithm 9, respectively. Specifically, these events are the following: an instub belonging to $v$ with label $g(v) \in\{C, D\}$ is 
sampled for pairing to an oustub of $v^{\prime}$ with label $g\left(v^{\prime}\right)=D$, or an instub $e$ with label $g(e)=0$ is sampled for pairing with any outstub.)

We begin with (63). First, observe that by definition $\mu_{s}^{(m)}\left(V_{n} \backslash K_{n}\right)$ and $v_{s}^{(m)}\left(V_{n} \backslash K_{n}\right)$ depend only the $m$-step neighborhood out of $s$ (i.e. the subgraph with nodes $\cup_{j=0}^{m} A_{j}$ ) in $G_{n}$ and $H_{n}$, respectively. When $\tau_{G}>m, U_{s}=1$ in Algorithm 5 and $\tau_{S}>m$ in Algorithm 9, these neighborhoods are constructed by the same procedure. Thus, (63) follows.

We next consider (65), which holds by a similar argument. Specifically, the left and right sides of (65) depend on the first $m$ generations of $\hat{G}_{n}$ and $\hat{H}_{n}$, respectively. In Algorithm 6, these first $m$ generations of $\hat{G}_{n}$ are constructed as follows: the root node $\phi$ has attributes $\left(N_{\phi}, D_{\phi}\right) \sim f_{n}^{*}$ and $U_{\phi}=1$, non-root nodes $\mathbf{i}$ have attributes $\left(N_{\mathbf{i}}, D_{\mathbf{i}}, U_{\mathbf{i}}\right) \sim f_{n}$, and $D_{\mathbf{i}}$ offspring are born to $\mathbf{i}$ if and only if $U_{\mathbf{i}}=1$. In Algorithm 9, the root node in $\hat{H}_{n}$ also has attributes has attributes $\left(N_{\phi}, D_{\phi}\right) \sim f_{n}^{*}$ and $U_{\phi}=1$; furthermore, with $\tau_{S}>m$, non-root nodes $\mathbf{i}$ have attributes $\left(N_{\mathbf{i}}, D_{\mathbf{i}}, U_{\mathbf{i}}\right) \sim f_{n}$ and $D_{\mathbf{i}}$ offspring are born for either value of $U_{\mathbf{i}}$. Hence, when $\tau_{S}>m$, modifying the construction of the first $m$ generations of $\hat{H}_{n}$ such that offspring are born only when $U_{\mathbf{i}}=1$ yields the construction of the first $m$ generations of $\hat{G}_{n}$. But, by (62), the left side of (65) remains unchanged when this modification occurs. Therefore, (65) follows.

It only remains to prove (64). For this, we begin with two lemmas. These lemmas use the mapping $\Phi$ from graph nodes to tree nodes defined in Algorithm 9 in Appendix D.6. Lemma 7 states that tree nodes that do not map back to graph nodes do not contribute to the right side of (64). Lemma 8 states that a tree node that does map back to a graph node contributes to the right side of (64) the same value that the corresponding graph node contributes to the left side of (64). Taken together, these lemmas will allow us to prove the lemma.

Lemma 7. If $\tau_{S}>m, \mathbf{i} \in \hat{A}_{j}$ for some $j \in\{0,1, \ldots, m\}$, and $\Phi^{-1}(\mathbf{i})=\emptyset$, then $U_{\mathbf{i}} \hat{v}_{\phi}(\mathbf{i})=0$.

Proof. We will denote $\mathbf{i}$ by $\mathbf{i}=\left(i_{1}, i_{2}, \ldots, i_{j}\right)$, and for $l \leq j$, we let $\mathbf{i} \mid l=\left(i_{1}, i_{2}, \ldots, i_{l}\right)$, with $\mathbf{i} \mid 0=\phi$ by convention. Define $l^{*}=\max \left\{l \in\{0,1, \ldots, j\}: \Phi^{-1}(\mathbf{i} \mid l) \neq \emptyset\right\}$. Note the set over which the maximum is taken is nonempty, since $\Phi^{-1}(\mathbf{i} \mid 0)=\Phi^{-1}(\phi)=s$; furthermore, since $\Phi^{-1}(\mathbf{i} \mid j)=\Phi^{-1}(\mathbf{i})=\emptyset$ by assumption, $l^{*}<j$. In words, $\mathbf{i} \mid l^{*}$ is the youngest ancestor of $\mathbf{i}$ that maps to a node in the tree; we let $v^{\prime}=\Phi^{-1}\left(\mathbf{i} \mid l^{*}\right)$ denote this node.

We observe $\Phi^{-1}(\mathbf{i} \mid l) \neq \emptyset \forall l \in\left\{0,1, \ldots, l^{*}-1\right\}$. To see this, suppose instead that $\Phi^{-1}(\mathbf{i} \mid l)=\emptyset$ for some such $l$. Then, from the second inner for loop in Algorithm 9, the offspring $\mathrm{i} \mid(l+1)$ was born without adding a node to the graph, which implies $\Phi^{-1}(\mathbf{i} \mid(l+1))=\emptyset$. Repeating this argument eventually gives $\Phi^{-1}\left(\mathbf{i} \mid l^{*}\right)=\emptyset$, a contradiction.

Now suppose $U_{\mathbf{i}} \hat{v}_{\phi}(\mathbf{i})>0$; we seek a contradiction. First, by (62), $U_{\mathbf{i}} \hat{v}_{\phi}(\mathbf{i})>0$ implies

$$
U_{\mathbf{i} \mid 0}=U_{\mathbf{i} \mid 1}=\cdots=U_{\mathbf{i}}=1
$$

which further implies $U_{\Phi^{-1}(\mathbf{i} \mid l)}=U_{\mathrm{i} \mid l}=1 \forall l \in\left\{0,1, \ldots, l^{*}\right\}$, i.e. the graph $H_{n}$ contains a path of length $l^{*}$ from $s=\Phi^{-1}(\mathbf{i} \mid 0)$ to $v^{\prime}=\Phi^{-1}\left(\mathbf{i} \mid l^{*}\right)$ that avoids $K_{n}$.

Next, note that $\Phi^{-1}\left(\mathbf{i} \mid l^{*}\right) \neq \emptyset, \Phi^{-1}\left(\mathbf{i} \mid\left(l^{*}+1\right)\right)=\emptyset$ implies that, during the $\left(l^{*}+1\right)$-th iteration of Algorithm 9, an outstub of $v^{\prime}$ was paired with an instub of some $v \in V_{n}$ that already belonged to the graph, and so a copy of $v$ (namely, $\mathbf{i} \mid\left(l^{*}+1\right)$ ) was added to the tree. Consider the following cases for the labels of these nodes at the moment of pairing:

- If $g\left(v^{\prime}\right)=A$ or $g(v)=A$, we have a contradiction, since by assumption, both $v^{\prime}$ and $v$ already belonged to the graph at the moment of pairing.

- If $g\left(v^{\prime}\right)=B$ or $g(v)=B, U_{\mathbf{i} \mid l^{*}}=U_{v^{\prime}}=0$ or $U_{\mathbf{i} \mid\left(l^{*}+1\right)}=U_{v}=0$, contradicting (66).

- If $g\left(v^{\prime}\right)=D, g(v) \in\{C, D\}$, then $\tau_{S}=l^{*} \leq m$ in Algorithm 9, a contradiction. 
The only remaining case is $g\left(v^{\prime}\right)=C$ at the moment of pairing. But this contradicts the earlier statement that the graph contains a path from $s$ to $v^{\prime}$ of length $l^{*}$ that avoids $K_{n}$ (since this path was present at start of the $\left(l^{*}+1\right)$-th iteration, it was present at the moment of pairing).

LEMMA 8. If $\tau_{S}>m$, then $U_{v} v_{s}^{(m)}(v)=U_{\Phi(v)} \hat{v}_{\phi}(\Phi(v)) \forall v \in \cup_{j=0}^{m} A_{j}$.

Proof. We proceed by induction. For the base of induction, we note $A_{0}=\{s\}$, so the statement only needs to be verified for $v=s$. But this is immediate, since $\Phi(s)=\phi$ and $U_{s}=U_{\phi}=1$ in Algorithm 9, and since $v_{s}^{(0)}(s)=\hat{v}_{\phi}(\phi)=1$ by (61) and (62).

Now assume $\tau_{S}>m$ and let $v \in \cup_{j=0}^{m} A_{j}$. We consider two cases.

First, if $v \in A_{j}$ for some $j \in\{0,1, \ldots, m-1\}$, we can use the inductive hypothesis to write

$$
U_{v} v_{s}^{(m)}(v)=U_{v}\left(v_{s}^{(m)}(v)-v_{s}^{(m-1)}(v)\right)+U_{v} v_{s}^{(m-1)}(v)=U_{v} e_{s}^{\top}\left(1-\alpha_{n}\right)^{m} \tilde{Q}^{m} e_{v}+U_{\Phi(v)} \hat{v}_{\phi}(\Phi(v)),
$$

and so it suffices to show $U_{v} e_{s}^{\top} \tilde{Q}^{m} e_{v}=0$. Clearly, this holds when $U_{v}=0$. If instead $U_{v}=1$, suppose $e_{s}^{\top} \tilde{Q}^{m} e_{v}>0$. First, note that $U_{v}=1$ and $v \in A_{j}, j<m$ imply $g(v) \in\{C, D\}$ at the start of the $m$-th iteration of Algorithm 9. Furthermore, $e_{s}^{\top} \tilde{Q}^{m} e_{v}>0$ implies there exists a path of length $m$ from $s$ to $v$, with every node $w$ along the path satisfying $U_{w}=1$. Let $v^{\prime}$ be the node immediately preceding $v$ on this path, so that an outstub of $v^{\prime}$ was paired with instub of $v$ during the $m$-th iteration. Then we have $e_{s}^{\top} \tilde{Q}^{m-1} e_{v^{\prime}}>0$, which implies $g\left(v^{\prime}\right)=D$ at the start of the $m$-th iteration of Algorithm 9. But $g\left(v^{\prime}\right)=D, g(v) \in\{C, D\}$ contradicts $\tau_{S}>m$ in Algorithm 9. Therefore, we must have $e_{s}^{\top} \tilde{Q}^{m} e_{v}=0$.

Now suppose $v \in A_{m}$. Then $U_{v} v_{s}^{(m-1)}(v)=0$ (else, $v$ is at most $m-1$ steps from $s$, contradicting $\left.v \in A_{m}\right)$, so we aim to show $U_{v} e_{s}^{\top}\left(1-\alpha_{n}\right)^{m} \tilde{Q}^{m}=U_{\Phi(v)} \hat{v}_{\phi}(\Phi(v))$. Since $U_{v}=U_{\Phi(v)}$ in Algorithm 9, this is trivial when $U_{v}=0$, and when $U_{v}=1$, it suffices to show

$$
e_{s}^{\top}\left(1-\alpha_{n}\right)^{m} \tilde{Q}^{m}=\hat{v}_{\phi}(\Phi(v)) \text {. }
$$

Towards this end, let $v^{\prime} \in \cup_{j=0}^{m-1} A_{j}$ be the first node whose outstub was paired with an instub of $v$ during the $m$-th iteration (which occurs by assumption $v \in A_{m}$ ); by the inductive hypothesis,

$$
U_{v^{\prime}} v_{s}^{(m-1)}\left(v^{\prime}\right)=U_{\Phi\left(v^{\prime}\right)} \hat{v}_{\phi}\left(\Phi\left(v^{\prime}\right)\right) .
$$

Now since $D_{v^{\prime}}=D_{\Phi\left(v^{\prime}\right)}$, and since $\Phi(v)$ is an offspring of $\Phi\left(v^{\prime}\right)$, we can use (62) to obtain

$$
\frac{\left(1-\alpha_{n}\right) U_{v^{\prime}} v_{s}^{(m-1)}\left(v^{\prime}\right)}{D_{v^{\prime}}}=\frac{\left(1-\alpha_{n}\right) U_{\Phi\left(v^{\prime}\right)} \hat{v}_{\phi}\left(\Phi\left(v^{\prime}\right)\right)}{D_{\Phi\left(v^{\prime}\right)}}=\hat{v}_{\phi}(\Phi(v)) \text {. }
$$

Next, observe the left side of $(67)$ is at most $e_{s}^{\top}\left(1-\alpha_{n}\right)^{m} \tilde{Q}^{m}$ by (61), so we must show this inequality is actually an equality. Suppose instead that the inequality is strict. Then, later in the $m$-th iteration, we must have paired an outstub of some $v^{\prime \prime}$ s.t. $g\left(v^{\prime \prime}\right)=D$ with another instub of $v$. But $g(v) \in\{C, D\}$ after the $v^{\prime}$ outstub was paired with the $v$ instub, and $g\left(v^{\prime \prime}\right)=D, g(v) \in\{C, D\}$ contradicts $\tau_{S}>m$ in Algorithm 9.

Having established these lemmas, we turn to the proof of (64). Assume $\tau_{S}>m$. Observe that by Lines 20-21 of Algorithm 9, $\left\{\Phi(v): v \in A_{j}\right\} \subset \hat{A}_{j}$, so the right side of (64) satisfies

$$
\sum_{j=0}^{m} \sum_{\mathbf{i} \in \hat{A}_{j}} U_{\mathbf{i}} \hat{v}_{\phi}(\mathbf{i})=\sum_{j=0}^{m}\left(\sum_{\mathbf{i} \in \hat{A}_{j}: \Phi^{-1}(\mathbf{i})=\emptyset} U_{\mathbf{i}} \hat{v}_{\phi}(\mathbf{i})+\sum_{v \in A_{j}} U_{\Phi(v)} \hat{v}_{\phi}(\Phi(v))\right) .
$$


Now since $U_{\mathbf{i}} \hat{v}_{\phi}(\mathbf{i}) \geq 0$ by definition, Lemma 7 implies

$$
\sum_{\mathbf{i} \in \hat{A}_{j}: \Phi^{-1}(\mathbf{i})=\emptyset} U_{\mathbf{i}} \hat{v}_{\phi}(\mathbf{i})=0 \forall j \in\{0,1, \ldots, m\} .
$$

Furthermore, since $v_{s}^{(m)}(v)=0 \forall v \notin \cup_{j=0}^{m} A_{j}$ (which holds by (61)), Lemma 8 implies

$$
v_{s}^{(m)}\left(V_{n} \backslash K_{n}\right)=\sum_{j=0}^{m} \sum_{v \in A_{j}} U_{v} v_{s}^{(m)}(v)=\sum_{j=0}^{m} \sum_{v \in A_{j}} U_{\Phi(v) \hat{v}_{\phi}}(\Phi(v)) .
$$

Finally, combining the previous three equations yields (64).

\section{D.4 Proof of Lemma 5}

We begin with some initial definitions that will be used throughout the proof. Specifically, let $\zeta_{n}=\mathbb{E}_{n}\left[D_{\mathrm{i}}\right]$ and $\lambda_{n}=\mathbb{E}_{n}\left[N_{\mathrm{i}} U_{\mathrm{i}}\right]$, where $\left(N_{\mathrm{i}}, D_{\mathrm{i}}, U_{\mathrm{i}}\right) \sim f_{n}$ are the attributes for a non-root node in the tree. Then, conditioned on $\Omega_{n}$,

$$
\begin{aligned}
& \zeta_{n}=\frac{1}{L_{n}} \sum_{h=1}^{n} N_{h} D_{h}=\frac{\eta_{2}\left(1+O\left(n^{-\gamma}\right)\right)}{\eta_{1}\left(1+O\left(n^{-\gamma}\right)\right)}=\zeta\left(1+O\left(n^{-\gamma}\right)\right), \\
& \lambda_{n}=\frac{1}{L_{n}} \sum_{h=1}^{n} N_{h}^{2} U_{h}=\frac{\eta_{3}\left(1+O\left(n^{-\gamma}\right)\right)}{\eta_{1}\left(1+O\left(n^{-\gamma}\right)\right)}=\lambda\left(1+O\left(n^{-\gamma}\right)\right) .
\end{aligned}
$$

Similarly, let $\zeta_{n}^{*}=\mathbb{E}_{n}\left[D_{\phi}\right]$ and $\lambda_{n}^{*}=\mathbb{E}_{n}\left[N_{\phi}\right]$, where $\left(N_{\phi}, D_{\phi}\right) \sim f_{n}^{*}$ are the attributes for the root node of the tree, so that given $\Omega_{n}$,

$$
\zeta_{n}^{*}=\frac{1}{\sum_{h=1}^{n} U_{h}} \sum_{h=1}^{n} D_{h} U_{h}=\zeta^{*}\left(1+O\left(n^{-\gamma}\right)\right), \quad \lambda_{n}^{*}=\frac{1}{\sum_{h=1}^{n} U_{h}} \sum_{h=1}^{n} N_{h} U_{h}=\lambda^{*}\left(1+O\left(n^{-\gamma}\right)\right) .
$$

We now explain our approach for bounding $\mathbb{P}\left[\tau_{G} \leq m \mid U_{s}=1\right]$. First, observe that, conditioned on $U_{s}=1$, the graphs in Algorithms 5 and 9 (the graph and simultaneous constructions, respectively) are constructed by the same procedure until $\tau_{G}=m$ or $\tau_{S}=m$; further, $\tau_{G}$ is assigned in Algorithm 5 by the same procedure $\tau_{S}$ is assigned in Algorithm 9. This implies

$$
\mathbb{P}\left[\tau_{G} \leq m \mid U_{s}=1\right]=\mathbb{P}\left[\tau_{S} \leq m\right] .
$$

Next, for $i \in\{0,1\}$, define

$$
E_{i}=\left\{g(e)=i \text { at the moment } \tau_{S} \text { is assigned in Algorithm 9 } .\right.
$$

In other words, $E_{0}$ is the event that the coupling breaks because a paired instub was sampled, while $E_{1}$ is the event that the coupling breaks because an unpaired instub that forms an edge $v^{\prime} \rightarrow v$ s.t. $g\left(v^{\prime}\right)=D, g(v) \in\{C, D\}$ was sampled. Furthermore, for $l \in\{1,2, \ldots, m\}$, define

$$
\hat{Z}_{l}=\sum_{\mathbf{i} \in \hat{A}_{l-1}} D_{\mathbf{i}}
$$

which is the total number of outstubs in generation $l-1$ of the tree; note $\hat{Z}_{l}=\left|\hat{A}_{l}\right|$. Finally, let $\left\{y_{n}: n \in \mathbb{N}\right\}$ be a sequence tending to infinity (which we will choose later), and let

$$
F_{m}=\left\{\max _{1 \leq l \leq m} \frac{\hat{Z}_{l}}{\zeta^{l-1}} \leq \zeta^{*} y_{n}\right\}
$$


We can then use the previous four equations to write

$$
\mathbb{P}\left[\tau_{G} \leq m \mid U_{s}=1\right] \leq O\left(n^{-\delta}\right)+\mathbb{P}\left[F_{m}^{C} \mid \Omega_{n}\right]+\sum_{i=0}^{1} \sum_{l=1}^{m} \mathbb{P}\left[\tau_{S}=l, E_{i}, F_{m} \mid \Omega_{n}\right] .
$$

where we have also used $\mathbb{P}\left[\Omega_{n}^{C}\right]=O\left(n^{-\delta}\right)$ by Assumption 1. In the remainder of this appendix, we bound each term in (69).

D.4.1 $\mathbb{P}\left[F_{m}^{C} \mid \Omega_{n}\right]$ bound. First, note that $\left\{D_{\mathbf{i}}\right\}_{\mathbf{i} \in \hat{A}_{l-1}}$ are identically distributed and independent of $\hat{Z}_{l-1}=\left|\hat{A}_{l-1}\right|$. Hence,

$$
\mathbb{E}_{n}\left[\hat{Z}_{l}\right]=\mathbb{E}_{n}\left[\mathbb{E}_{n}\left[\hat{Z}_{l} \mid \hat{Z}_{l-1}\right]\right]=\mathbb{E}_{n}\left[\hat{Z}_{l-1} \mathbb{E}_{n}\left[D_{\mathbf{i}} \mid \hat{Z}_{l-1}\right]\right]=\mathbb{E}_{n}\left[\hat{Z}_{l-1}\right] \mathbb{E}_{n}\left[D_{\mathbf{i}}\right]=\mathbb{E}_{n}\left[\hat{Z}_{l-1}\right] \zeta_{n},
$$

and so applying recursively gives

$$
\mathbb{E}_{n}\left[\hat{Z}_{l}\right]=\mathbb{E}_{n}\left[\hat{Z}_{1}\right] \zeta_{n}^{l-1}=\mathbb{E}_{n}\left[D_{\phi}\right] \zeta_{n}^{l-1}=\zeta_{n}^{*} \zeta_{n}^{l-1}
$$

Now let $X_{l}=\hat{Z}_{l} /\left(\zeta_{n}^{*} \zeta_{n}^{l-1}\right)$, so that $\mathbb{E}_{n}\left[X_{l}\right]=1$. Furthermore, define

$$
\mathcal{G}_{l}=\sigma\left(\left\{N_{h}, D_{h}, U_{h}: 1 \leq h \leq n\right\} \cup\left\{D_{\mathbf{i}}: \mathbf{i} \in \hat{A}_{j}, 0 \leq j<l\right\}\right) .
$$

Then for $j>0$,

$$
\begin{aligned}
\mathbb{E}\left[X_{l+j} \mid \mathcal{G}_{l}\right] & =\frac{\mathbb{E}\left[\hat{Z}_{l+j} \mid \mathcal{G}_{l}\right]}{\zeta_{n}^{*} \zeta_{n}^{l+j-1}}=\frac{\mathbb{E}\left[\hat{Z}_{l+j-1} \mid \mathcal{G}_{l}\right] \mathbb{E}\left[D_{\mathrm{i}} \mid \mathcal{G}_{l}\right]}{\zeta_{n}^{*} \zeta_{n}^{l+j-1}}=\frac{\mathbb{E}\left[\hat{Z}_{l+j-1} \mid \mathcal{G}_{l}\right]}{\zeta_{n}^{*} \zeta_{n}^{l+j-2}} \\
& =\mathbb{E}\left[X_{l+j-1} \mid \mathcal{G}_{l}\right]=\cdots=\mathbb{E}\left[X_{l} \mid \mathcal{G}_{l}\right]=X_{l},
\end{aligned}
$$

so $\left\{X_{l}: l \in \mathbb{N}\right\}$ is a martingale with respect to $\left\{G_{l}: l \in \mathbb{N}\right\}$. This implies, by Doob's inequality,

$$
\mathbb{P}_{n}\left[\max _{1 \leq l \leq m} X_{l}>\frac{y_{n}}{\left(1+O\left(n^{-\gamma}\right)\right)^{m}}\right] \leq \frac{\left(1+O\left(n^{-\gamma}\right)\right)^{m}}{y_{n}},
$$

where we have used $\mathbb{E}_{n}\left[X_{m}\right]=1$. Using this bound, we can obtain

$$
\begin{aligned}
\mathbb{P}\left[F_{m}^{C} \mid \Omega_{n}\right] & =\mathbb{P}\left[\max _{1 \leq l \leq m} \frac{\hat{Z}_{l}}{\zeta^{l-1}}>\zeta^{*} y_{n} \mid \Omega_{n}\right]=\mathbb{P}\left[\max _{1 \leq l \leq m} \frac{X_{l} \zeta_{n}^{*} \zeta_{n}^{l-1}}{\zeta^{*} \zeta^{l-1}}>y_{n} \mid \Omega_{n}\right] \\
& =\mathbb{P}\left[\max _{1 \leq l \leq m} X_{l}\left(1+O\left(n^{-\gamma}\right)\right)^{l}>y_{n} \mid \Omega_{n}\right] \leq \mathbb{P}\left[\max _{1 \leq l \leq m} X_{l}>\frac{y_{n}}{\left(1+O\left(n^{-\gamma}\right)\right)^{m}} \mid \Omega_{n}\right] \\
& =\frac{1}{\mathbb{P}\left[\Omega_{n}\right]} \mathbb{E}\left[1\left(\Omega_{n}\right) \mathbb{P}_{n}\left[\max _{1 \leq l \leq m} X_{l}>\frac{y_{n}}{\left(1+O\left(n^{-\gamma}\right)\right)^{m}}\right]\right] \\
& \leq \frac{1}{\mathbb{P}\left[\Omega_{n}\right]} \mathbb{E}\left[1\left(\Omega_{n}\right) \frac{\left(1+O\left(n^{-\gamma}\right)\right)^{m}}{y_{n}}\right]=\frac{\left(1+O\left(n^{-\gamma}\right)\right)^{m}}{y_{n}}=O\left(y_{n}^{-1}\right),
\end{aligned}
$$

where in the third line we used the tower property and the fact that $1\left(\Omega_{n}\right)$ is fixed given the degree sequence, and where the final equality holds by the assumption $m=O\left(n^{\gamma}\right)$ in the statement of the lemma, since then $\left(1+O\left(n^{-\gamma}\right)\right)^{m}=\left(1+\frac{O(1)}{m}\right)^{m}=e^{O(1)}=O(1)$.

D.4.2 $\mathbb{P}\left[\tau_{S}=l, E_{0}, F_{m} \mid \Omega_{n}\right]$ bound. For $l \in\{1,2, \ldots, m\}$,

$$
\mathbb{P}\left[\tau_{S}=l, E_{0}, F_{m} \mid \Omega_{n}\right]=\mathbb{E}\left[1\left(F_{m}\right) \mathbb{P}_{n}\left[\tau_{S}=l, E_{0} \mid\left\{\hat{Z}_{j}\right\}_{j=1}^{m+1}\right] \mid \Omega_{n}\right]
$$

which holds because $1\left(\Omega_{n}\right)$ and $1\left(F_{m}\right)$ are fixed given the degree sequence and $\left\{\hat{Z}_{j}\right\}_{j=1}^{m}$. Next, observe $\left\{\tau_{S}=l, E_{0}\right\}$ occurs if and only if, during the $l$-th iteration, we sample an instub that has already been paired while attempting to pair an outstub belonging to a node $v^{\prime} \in A_{l-1}$. We aim to bound the probability of this event. 
Consider any such outstub. Since we sample instubs uniformly from the set of all $L_{n}$ instubs, the probability of sampling a paired instub is the fraction of paired instubs at the moment we attempt to pair the outstub under consideration. This fraction is clearly bounded by the fraction of paired instubs at the end of the $l$-th iteration. Further, since each time we pair an instub of $v \in V$ in the graph, we also add a node to the tree with the same attributes as $v$, the numerator of this fraction is further bounded by the number of nodes in the tree at the end of the $l$-th iteration, which is

$$
\frac{1}{L_{n}} \sum_{j=1}^{l+1} \hat{Z}_{j}
$$

where (we recall) $L_{n}=\sum_{v \in V_{n}} N_{v}=\sum_{v \in V_{n}} D_{v}$. Now consider the number of such outstubs. By definition, this is $\sum_{v^{\prime} \in A_{l-1}} D_{v^{\prime}}$. Furthermore, since each time we add a node to $A_{l-1}$ in the graph, we also add a node with the same attributes to $\hat{A}_{l-1}$ in the tree, we have the bound

$$
\sum_{v^{\prime} \in A_{l-1}} D_{v^{\prime}} \leq \sum_{\mathbf{i} \in \hat{A}_{l-1}} D_{\mathbf{i}} \triangleq \hat{Z}_{l}
$$

Combining these arguments, letting Bin denote a binomial random variable, and using Markov's inequality, we can write

$$
\begin{aligned}
\mathbb{P}_{n}\left[\tau_{S}=l, E_{0} \mid\left\{\hat{Z}_{j}\right\}_{j=1}^{m+1}\right] & \leq \mathbb{P}_{n}\left[\operatorname{Bin}\left(\hat{Z}_{l}, \frac{\sum_{j=1}^{l+1} \hat{Z}_{j}}{L_{n}}\right) \geq 1 \mid\left\{\hat{Z}_{j}\right\}_{j=1}^{m+1}\right] \\
& \leq \mathbb{E}_{n}\left[\operatorname{Bin}\left(\hat{Z}_{l}, \frac{\sum_{j=1}^{l+1} \hat{Z}_{j}}{L_{n}}\right) \mid\left\{\hat{Z}_{j}\right\}_{j=1}^{m+1}\right]=\hat{Z}_{l} \frac{\sum_{j=1}^{l+1} \hat{Z}_{j}}{L_{n}} .
\end{aligned}
$$

Next, we recognize $1\left(F_{m}\right) \hat{Z}_{l} \leq \zeta^{*} \zeta^{l-1} y_{n}$ by definition of $F_{m}$, and we combine (71) and (73),

$$
\mathbb{P}\left[\tau_{S}=l, E_{0}, F_{m} \mid \Omega_{n}\right] \leq \mathbb{E}\left[1\left(F_{m}\right) \hat{Z}_{l} \frac{\sum_{j=1}^{l+1} \hat{Z}_{j}}{L_{n}} \mid \Omega_{n}\right] \leq \zeta^{*} \zeta^{l-1} y_{n} \sum_{j=1}^{l+1} \mathbb{E}\left[\frac{\hat{Z}_{j}}{L_{n}} \mid \Omega_{n}\right] .
$$

Furthermore, by definition of $\Omega_{n}$, we have

$$
\mathbb{E}\left[\frac{\hat{Z}_{j}}{L_{n}} \mid \Omega_{n}\right]=\mathbb{E}\left[\frac{\mathbb{E}_{n}\left[\hat{Z}_{j}\right]}{L_{n}} \mid \Omega_{n}\right]=\mathbb{E}\left[\frac{\zeta_{n}^{*} \zeta_{n}^{j-1}}{L_{n}} \mid \Omega_{n}\right]=\frac{\zeta^{*} \zeta^{j-1}}{n \eta_{1}}\left(1+O\left(n^{-\gamma}\right)\right)^{j}=O\left(\frac{\zeta^{j-1}}{n}\right),
$$

where $\left(1+O\left(n^{-\gamma}\right)\right)^{j}=O(1)$ again follows from $m=O\left(n^{-\gamma}\right)$. We have therefore shown

$$
\mathbb{P}\left[\tau_{S}=l, E_{0}, F_{m} \mid \Omega_{n}\right]=O\left(\frac{y_{n}}{n} \zeta^{l-1} \sum_{j=0}^{l} \zeta^{j}\right) .
$$

D.4.3 $\mathbb{P}\left[\tau_{S}=l, E_{1}, F_{m} \mid \Omega_{n}\right]$ bound. We will use the same approach to bound this term as we used to bound the previous one. Observe $\left\{\tau_{S}=l, E_{1}\right\}$ occurs if and only if, during the $l$-th iteration, we sample an instub belonging to $v$ s.t. $g(v) \in\{C, D\}$ while attempting to pair an outstub belonging to a node $v^{\prime} \in A_{l-1}$ s.t. $g\left(v^{\prime}\right)=D$. The key step in the derivation will be bounding the number of such instubs and outstubs.

First, the number of such outstubs is clearly bounded the number of all outstubs paired during the $l$-th iteration. As we argued previously, this is further bounded by $\hat{Z}_{l}$. Next, for $j \in\{1,2, \ldots, m+1\}$, define $\hat{V}_{j}=\sum_{\mathbf{i} \in \hat{A}_{j-1}} N_{\mathbf{i}} U_{\mathbf{i}}$. Similar to the previous argument, the number of such instubs while pairing any such outstub is bounded by the number of instubs belonging to $U_{v}=1$ nodes in the graph at the end of the $l$-th iteration. Since each time we add a node to the graph, we also add a 
node to the tree with the same attributes, the former quantity is bounded by the same quantity computed on the tree, i.e.

$$
\sum_{j=1}^{l+1} \sum_{\mathbf{i} \in \hat{A}_{l-1}} N_{\mathbf{i}} U_{\mathbf{i}}=\sum_{j=1}^{l+1} \hat{V}_{j}
$$

Hence, as in the analysis of $\mathbb{P}\left[\tau_{S}=l, E_{0}, F_{m} \mid \Omega_{n}\right]$,

$$
\begin{aligned}
\mathbb{P}\left[\tau_{S}=l, E_{1}, F_{m} \mid \Omega_{n}\right] & =\mathbb{E}\left[1\left(F_{m}\right) \mathbb{P}_{n}\left[\tau_{S}=l, E_{1} \mid\left\{\hat{Z}_{j}\right\}_{j=1}^{m},\left\{\hat{V}_{j}\right\}_{j=1}^{l+1}\right] \mid \Omega_{n}\right] \\
& \leq \mathbb{E}\left[1\left(F_{m}\right) \hat{Z}_{l} \frac{\sum_{j=1}^{l+1} \hat{V}_{j}}{L_{n}} \mid \Omega_{n}\right] \leq \zeta^{*} \zeta^{l-1} y_{n} \sum_{j=1}^{l+1} \mathbb{E}\left[\frac{\mathbb{E}_{n}\left[\hat{V}_{j}\right]}{L_{n}} \mid \Omega_{n}\right]
\end{aligned}
$$

Our final step is to compute $\mathbb{E}_{n}\left[\hat{V}_{j}\right]$. For $j>1$, we have

$$
\mathbb{E}_{n}\left[\hat{V}_{j}\right]=\mathbb{E}_{n}\left[\hat{Z}_{j-1}\right] \mathbb{E}_{n}\left[N_{\mathrm{i}} U_{\mathrm{i}}\right]=\zeta_{n}^{*} \zeta_{n}^{j-2} \lambda_{n}
$$

where the first equality holds since $\left|\hat{A}_{j-1}\right|=\hat{Z}_{j-1}$ and since $\left\{N_{\mathbf{i}} U_{\mathbf{i}}: \mathbf{i} \in \hat{A}_{l-1}\right\}$ are identically distributed and independent of $\hat{Z}_{j-1}$; the second equality follows from previous derivations. Therefore,

$$
\mathbb{E}\left[\frac{\mathbb{E}_{n}\left[\hat{V}_{j}\right]}{L_{n}} \mid \Omega_{n}\right]=\mathbb{E}\left[\frac{\zeta_{n}^{*} \zeta_{n}^{j-2} \lambda_{n}}{L_{n}} \mid \Omega_{n}\right]=\frac{\zeta^{*} \zeta^{j-2} \lambda}{n \eta_{1}}\left(1+O\left(n^{-\gamma}\right)\right)^{j}=O\left(\frac{\zeta^{j-2}}{n}\right) .
$$

For $j=1$, since $\hat{A}_{0}=\{\phi\}$ with $U_{\phi}=1$, we simply have $\mathbb{E}_{n}\left[\hat{V}_{1}\right]=\mathbb{E}_{n}\left[N_{\phi}\right]=\lambda_{n}^{*}$, so

$$
\mathbb{E}\left[\frac{\mathbb{E}_{n}\left[\hat{V}_{1}\right]}{L_{n}} \mid \Omega_{n}\right]=\mathbb{E}\left[\frac{\lambda_{n}^{*}}{L_{n}} \mid \Omega_{n}\right]=\frac{\lambda^{*}}{n \eta_{1}}\left(1+O\left(n^{-\gamma}\right)\right)=O\left(\frac{1}{n}\right) .
$$

Combining previous arguments, we obtain

$$
\mathbb{P}\left[\tau_{S}=l, E_{1}, F_{m} \mid \Omega_{n}\right]=O\left(\frac{y_{n}}{n} \zeta^{l-1} \sum_{j=0}^{l-1} \zeta^{j}\right) .
$$

D.4.4 Overall bound. Combining the bounds from the previous sections, we obtain

$$
\mathbb{P}\left[\tau_{G} \leq m \mid U_{s}=1\right]=O\left(n^{-\delta}+y_{n}^{-1}+\frac{y_{n}}{n} \sum_{l=1}^{m} \zeta^{l-1} \sum_{j=0}^{l} \zeta^{j}\right) .
$$

By Assumption 1, we have $\zeta>1$, which implies

$$
\sum_{l=1}^{m} \zeta^{l-1} \sum_{j=0}^{l} \zeta^{j}=\sum_{l=1}^{m} \zeta^{l-1} \frac{\zeta^{l+1}-1}{\zeta-1} \leq \frac{1}{\zeta-1} \sum_{l=1}^{m} \zeta^{2 l}=\frac{\zeta^{2}\left(\zeta^{2 m}-1\right)}{(1-\zeta)^{2}} \leq\left(\frac{\zeta}{\zeta-1}\right)^{2} \zeta^{2 m}=O\left(\zeta^{2 m}\right) .
$$

We thus obtain

$$
\mathbb{P}\left[\tau_{G} \leq m \mid U_{s}=1\right]=O\left(n^{-\delta}+y_{n}^{-1}+y_{n} \zeta^{2 m} / n\right)
$$

Finally, we choose $y_{n}$ to minimize the bound. This yields

$$
\mathbb{P}\left[\tau_{G} \leq m \mid U_{s}=1\right]=O\left(n^{-\delta}+\zeta^{m} / \sqrt{n}\right),
$$

which is what we set out to prove. 
D.4.5 Remark. Towards bounding $\mathbb{P}\left[\tau_{S}=l, E_{1}, F_{m} \mid \Omega_{n}\right]$, we bounded the number of outstubs belonging to $v^{\prime} \in A_{l-1}$ s.t. $g\left(v^{\prime}\right)=D$ by $\hat{Z}_{l}$. We can in fact obtain a tighter bound, suggesting that the bound above was unnecessarily loose. However, we show here that this modified approach ultimately yields the same result. For this, suppose in Assumption 1 we add the event

$$
\Omega_{n, 7}=\left\{\left|\frac{\sum_{h=1}^{n} N_{h} D_{h} U_{h}}{n}-\eta_{4}\right| \leq n^{-\gamma}\right\},
$$

and we set $\zeta^{\prime}=\eta_{4} / \eta_{1}$. Let $\left\{y_{n}^{\prime}: n \in \mathbb{N}\right\}$ be a sequence tending to infinity, and define

$$
\hat{Z}_{l}^{\prime}=\sum_{\mathbf{i} \in \hat{A}_{l-1}}\left(\prod_{j=0}^{l-1} U_{\mathbf{i} \mid j}\right) D_{\mathbf{i}}, \quad F_{m}^{\prime}=\left\{\max _{1 \leq l \leq m} \frac{\hat{Z}_{l}^{\prime}}{\left(\zeta^{\prime}\right)^{l-1}} \leq \zeta^{*} y_{n}^{\prime}\right\} .
$$

Using the same approach we used to bound $\mathbb{P}\left[F_{m}^{C} \mid \Omega_{n}\right]$, it is possible to show

$$
\mathbb{P}\left[\left(F_{m}^{\prime}\right)^{C} \mid \Omega_{n}\right]=O\left(\left(y_{n}^{\prime}\right)^{-1}\right) .
$$

Then, when analyzing $\left\{\tau_{S}=l, E_{1}\right\}$, we could bound the number of outstubs belonging to $v^{\prime} \in A_{l-1}$ s.t. $g\left(v^{\prime}\right)=D$ by $\hat{Z}_{l}^{\prime}$, which is tighter than $\hat{Z}_{l}$ (used above). This would ultimately yield

$$
\mathbb{P}\left[\tau_{S}=l, E_{1}, F_{m}^{\prime} \mid \Omega_{n}\right]=O\left(\frac{y_{n}^{\prime}}{n}\left(\zeta^{\prime}\right)^{l-1} \sum_{j=0}^{l-1} \zeta^{j}\right),
$$

which would imply

$$
\mathbb{P}\left[\tau_{S} \leq m, E_{1}, F_{m}^{\prime} \mid \Omega_{n}\right]=O\left(\frac{y_{n}^{\prime}}{n} \sum_{l=1}^{m}\left(\zeta^{\prime}\right)^{l-1} \sum_{j=0}^{l-1} \zeta^{j}\right)=O\left(\frac{y_{n}^{\prime}}{n}\left(\zeta \zeta^{\prime}\right)^{m}\right) .
$$

Using this approach, we could write

$$
\begin{aligned}
\mathbb{P} & \left.\tau_{G} \leq m \mid U_{s}=1\right] \\
& =O\left(n^{-\delta}\right)+\mathbb{P}\left[F_{m}^{C} \mid \Omega_{n}\right]+\mathbb{P}\left[\tau_{S} \leq m, E_{0}, F_{m} \mid \Omega_{n}\right]+\mathbb{P}\left[\left(F_{m}^{\prime}\right)^{C} \mid \Omega_{n}\right]+\mathbb{P}\left[\tau_{S} \leq m, E_{1}, F_{m}^{\prime} \mid \Omega_{n}\right] \\
& =O\left(n^{-\delta}+y_{n}^{-1}+\frac{y_{n}}{n} \zeta^{2 m}+\left(y_{n}^{\prime}\right)^{-1}+\frac{y_{n}^{\prime}}{n}\left(\zeta \zeta^{\prime}\right)^{m}\right)=O\left(n^{-\delta}+\frac{\zeta^{m}}{\sqrt{n}}+\frac{\left(\zeta \zeta^{\prime}\right)^{m / 2}}{\sqrt{n}}\right),
\end{aligned}
$$

where in the final step we chose $y_{n}, y_{n}^{\prime}$ to minimize the bound. However, since $\zeta^{\prime} \leq \zeta$ by definition, this bound is ultimately $O\left(n^{-\delta}+\zeta^{m} / \sqrt{n}\right)$, which is the same bound we obtained above.

\section{D.5 Proof of Lemma 6}

For $j \in\{1,2, \ldots, m\}$, let $X_{j}=\sum_{\mathbf{i} \in \hat{A}_{j}} U_{\mathbf{i}} \hat{\mu}_{\phi}(\mathbf{i})$, and for $n \in \mathbb{N}$, let

$$
\hat{p}_{n}=\frac{\sum_{h=1}^{n} U_{h} N_{h}}{L_{n}} .
$$

Note that, by Assumption 1, $\left|\hat{p}_{n}-p\right|<n^{-\gamma}$ when $\Omega_{n}$ holds.

Before proceeding, we present some intermediate results required for our analysis.

Lemma 9. For any $i, j \in \mathbb{N}$ s.t. $j \geq i$, let $X^{i}=\left\{X_{l}\right\}_{l=1}^{i}$. Then $\mathbb{E}_{n}\left[X_{j} \mid X^{i}\right]=\left(\left(1-\alpha_{n}\right) \hat{p}_{n}\right)^{j-i} X_{i}$.

Proof. We first observe

$$
X_{j}=\sum_{\mathbf{i} \in \hat{A}_{j}} U_{\mathbf{i}} \hat{\mu}_{\phi}(\mathbf{i})=\sum_{\mathbf{i} \in \hat{A}_{j}} \prod_{l=0}^{j-1} \frac{\left(1-\alpha_{n}\right) U_{\mathbf{i} \mid l}}{D_{\mathbf{i} \mid l}} U_{\mathbf{i}}=\sum_{\mathbf{i} \in \hat{A}_{j-1}} \prod_{l=0}^{j-1} \frac{\left(1-\alpha_{n}\right) U_{\mathbf{i} \mid l}}{D_{\mathbf{i} \mid l}} \sum_{k=1}^{D_{\mathbf{i}}} U_{(\mathbf{i}, k)},
$$


where the first equality follows from (22) and the second follows since, by Algorithm 6,

$$
\hat{A}_{j}=\left\{(\mathbf{i}, k): \mathbf{i} \in \hat{A}_{j-1}, U_{\mathbf{i}}=1, k \in\left\{1,2, \ldots, D_{\mathbf{i}}\right\}\right\} .
$$

Next, let $\mathbf{i} \in \hat{A}_{j-1}$ s.t. $U_{\mathbf{i}}=1$. For each $k \in\left\{1,2, \ldots, D_{\mathbf{i}}\right\}$, observe

$$
\begin{gathered}
\mathbb{E}\left[U_{(\mathrm{i}, k)} \mid\left\{N_{h}, D_{h}, U_{h}: 1 \leq h \leq n\right\} \cup\left\{U_{\mathrm{i}^{\prime}}, D_{\mathrm{i}^{\prime}}: \mathbf{i}^{\prime} \in \hat{A}_{s}, s<j\right\}\right] \\
=\mathbb{E}\left[U_{(\mathrm{i}, k)} \mid\left\{N_{h}, D_{h}, U_{h}: 1 \leq h \leq n\right\}\right]=\frac{\sum_{h=1}^{n} U_{h} N_{h}}{L_{n}}=\hat{p}_{n},
\end{gathered}
$$

which follows since in Algorithm 6, $\left(N_{(\mathrm{i}, k)}, D_{(\mathrm{i}, k)}, U_{(\mathrm{i}, k)}\right)$ are sampled from $f_{n}$, independent of the attributes of nodes in previous generations. Combining (75) and (76) gives

$$
\begin{aligned}
\mathbb{E} & {\left[X_{j} \mid\left\{N_{h}, D_{h}, U_{h}: 1 \leq h \leq n\right\} \cup\left\{U_{\mathbf{i}}, D_{\mathbf{i}}: \mathbf{i} \in \hat{A}_{s}, s<j\right\}\right] } \\
= & \sum_{\mathbf{i} \in \hat{A}_{j-1}} \prod_{l=0}^{j-1} \frac{\left(1-\alpha_{n}\right) U_{\mathbf{i} \mid l}}{D_{\mathbf{i} \mid l}} \sum_{k=1}^{D_{\mathbf{i}}} \hat{p}_{n}=\sum_{\mathbf{i} \in \hat{A}_{j-1}} \prod_{l=0}^{j-2} \frac{\left(1-\alpha_{n}\right) U_{\mathbf{i} \mid l}}{D_{\mathbf{i} \mid l}} \frac{\left(1-\alpha_{n}\right) U_{\mathbf{i}}}{D_{\mathbf{i}}}\left(D_{\mathbf{i}} \hat{p}_{n}\right) \\
& =\left(1-\alpha_{n}\right) \hat{p}_{n} \sum_{\mathbf{i} \in \hat{A}_{j-1}} \prod_{l=0}^{j-2} \frac{\left(1-\alpha_{n}\right) U_{\mathbf{i} \mid l}}{D_{\mathbf{i} \mid l}} U_{\mathbf{i}}=\left(1-\alpha_{n}\right) \hat{p}_{n} \sum_{\mathbf{i} \in \hat{A}_{j-1}} \hat{\mu}_{\phi}(\mathbf{i}) U_{\mathbf{i}}=\left(1-\alpha_{n}\right) \hat{p}_{n} X_{j-1} .
\end{aligned}
$$

Note that $X^{i}$ is a function of $\left\{U_{\mathbf{i}}, D_{\mathbf{i}}: \mathbf{i} \in \hat{A}_{s}, s<j\right\}$, so we can also write

$$
\mathbb{E}\left[X_{j} \mid\left\{N_{h}, D_{h}, U_{h}: 1 \leq h \leq n\right\} \cup\left\{U_{\mathbf{i}}, D_{\mathbf{i}}: \mathbf{i} \in \hat{A}_{s}, s<j\right\} \cup X^{i}\right]=\left(1-\alpha_{n}\right) \hat{p}_{n} X_{j-1} .
$$

Then, taking conditional expectation with respect to $\left\{N_{h}, D_{h}, U_{h}: 1 \leq h \leq n\right\} \cup X^{i}$ on both sides,

$$
\mathbb{E}_{n}\left[X_{j} \mid X^{i}\right]=\left(1-\alpha_{n}\right) \hat{p}_{n} \mathbb{E}_{n}\left[X_{j-1} \mid X^{i}\right],
$$

and so applying recursively gives

$$
\mathbb{E}_{n}\left[X_{j} \mid X^{i}\right]=\left(\left(1-\alpha_{n}\right) \hat{p}_{n}\right)^{j-i} X_{i}
$$

which completes the proof.

LEMMA 10. Let $Z$ be a random variable satisfying $\mathbb{E}[Z]=0$ and $a \leq Z \leq b$ a.s. Then

$$
\mathbb{E}\left[e^{\lambda Z}\right] \leq e^{\lambda^{2}(b-a)^{2} / 8} \forall \lambda>0 .
$$

Proof. See, for example, Lemma 5.1 in [24].

Lemma 11. For any $j \in \mathbb{N}$ and any $c_{j}>0$, define $\left.Y_{j}=c_{j}\left(X_{j}-\left(1-\alpha_{n}\right) \hat{p}_{n} X_{j-1}\right)\right)$. Then

$$
\mathbb{E}_{n}\left[\exp \left(\lambda Y_{j}\right) \mid X_{j-1}\right] \leq \exp \left(\frac{\lambda^{2}}{8}\left(c_{j}\left(1-\alpha_{n}\right)^{j}\right)^{2}\right)
$$

Proof. Note $\mathbb{E}_{n}\left[Y_{j} \mid X_{j-1}\right]=0$ by Lemma 9. Furthermore, $X_{j} \in\left[0,\left(1-\alpha_{n}\right) X_{j-1}\right]$ by (22), so

$$
Y_{j} \leq c_{j}\left(1-\alpha_{n}\right)\left(1-\hat{p}_{n}\right) X_{j-1} \triangleq b_{j}, \quad Y_{j} \geq-c_{j}\left(1-\alpha_{n}\right) \hat{p}_{n} X_{j-1} \triangleq a_{j} .
$$

Therefore, applying Lemma 10 gives

$$
\mathbb{E}_{n}\left[\exp \left(\lambda Y_{j}\right) \mid X_{j-1}\right] \leq \exp \left(\frac{\lambda^{2}}{8}\left(c_{j}\left(1-\alpha_{n}\right) X_{j-1}\right)^{2}\right),
$$

and using $X_{j-1} \leq\left(1-\alpha_{n}\right)^{j-1}$ (which again follows from (22)) completes the proof. 
We now turn to the proof of the lemma. First, we write

$$
\mathbb{P}\left[\alpha_{n} \sum_{j=1}^{m-1} X_{j}+X_{m} \geq \epsilon\right] \leq \mathbb{P}\left[\alpha_{n} \sum_{j=1}^{m-1} X_{j}+X_{m} \geq \epsilon \mid \Omega_{n}\right]+\mathbb{P}\left[\Omega_{n}^{C}\right] .
$$

Recall $\mathbb{P}\left[\Omega_{n}^{C}\right]=O\left(n^{-\delta}\right)$ by Assumption 1 , so it remains to bound the first summand. First,

$$
\mathbb{P}\left[\alpha_{n} \sum_{j=1}^{m-1} X_{j}+X_{m} \geq \epsilon \mid \Omega_{n}\right]=\frac{1}{\mathbb{P}\left[\Omega_{n}\right]} \mathbb{E}\left[1\left(\Omega_{n}\right) \mathbb{P}_{n}\left[\alpha_{n} \sum_{j=1}^{m-1} X_{j}+X_{m} \geq \epsilon\right]\right] .
$$

For the term inside the expectation, we have

$$
\mathbb{P}_{n}\left[\alpha_{n} \sum_{j=1}^{m-1} X_{j}+X_{m}>\epsilon\right] \leq \mathbb{P}_{n}\left[\alpha_{n} \sum_{j=1}^{m-1} X_{j}>\frac{\epsilon}{2}\right]+\mathbb{P}_{n}\left[X_{m}>\frac{\epsilon}{2}\right] .
$$

For the second summand, we use Markov's inequality to write

$$
\mathbb{P}_{n}\left[X_{m}>\frac{\epsilon}{2}\right] \leq \frac{2 \mathbb{E}_{n}\left[X_{m}\right]}{\epsilon}=\frac{2\left(1-\alpha_{n}\right)^{m} \hat{p}_{n}^{m}}{\epsilon}<\frac{2 \hat{p}_{n}^{m}}{\epsilon} .
$$

Recall that $\hat{p}_{n} \leq p+n^{-\gamma}$ when $\Omega_{n}$ holds. Therefore, by assumption $m=O\left(n^{\gamma}\right)$, we obtain

$$
1\left(\Omega_{n}\right) \mathbb{P}_{n}\left[X_{m}>\frac{\epsilon}{2}\right]<\frac{2\left(p+n^{-\gamma}\right)^{m}}{\epsilon}=p^{m} \frac{2\left(1+\frac{1 / p}{n^{\gamma}}\right)^{m}}{\epsilon}=O\left(p^{m}\right) .
$$

We next consider the first summand in (79). First, we use the Chernoff bound to write

$$
\mathbb{P}_{n}\left[\alpha_{n} \sum_{j=1}^{m-1} X_{j}>\epsilon\right] \leq \min _{\lambda>0} e^{-\lambda \epsilon} \mathbb{E}_{n}\left[\prod_{j=1}^{m-1} \exp \left(\lambda \alpha_{n} X_{j}\right)\right]
$$

To analyze (81), we require a definition: for $j=0,1, \ldots, m-1$, let

$$
c_{j}=\mathbb{E}_{n}\left[\alpha_{n} \sum_{i=0}^{m-j-1} X_{i}\right]=\alpha_{n} \sum_{i=0}^{m-j-1}\left(\left(1-\alpha_{n}\right) \hat{p}_{n}\right)^{i}=\frac{\alpha_{n}\left(1-\left(\left(1-\alpha_{n}\right) \hat{p}_{n}\right)^{m-j}\right)}{1-\left(1-\alpha_{n}\right) \hat{p}_{n}},
$$

where we have used Lemma 9 and since, by definition,

$$
X_{0}=\sum_{\mathbf{i} \in \hat{A}_{0}} U_{\mathbf{i}} \mu_{\phi}(\mathbf{i})=U_{\phi} \mu_{\phi}(\phi)=1 .
$$

From (82), it is straightforward to show

$$
c_{0}-\alpha_{n}=\mathbb{E}_{n}\left[\alpha_{n} \sum_{i=1}^{m-1} X_{i}\right], \quad c_{m-1}=\alpha_{n}, \quad c_{j}=\alpha_{n}+\left(1-\alpha_{n}\right) \hat{p}_{n} c_{j+1} .
$$

Now for $j \in\{1, \ldots, m-1\}$, we use Lemma 11 and (83) to obtain

$$
\begin{aligned}
\mathbb{E}_{n}\left[\exp \left(\lambda c_{j} X_{j}\right) \mid X^{j-1}\right] & =\mathbb{E}_{n}\left[\exp \left(\lambda c_{j}\left(X_{j}-\left(1-\alpha_{n}\right) \hat{p}_{n} X_{j-1}\right)\right) \mid X^{j-1}\right] \exp \left(\lambda c_{j}\left(1-\alpha_{n}\right) \hat{p}_{n} X_{j-1}\right) \\
& \leq \exp \left(\frac{\lambda^{2}}{8}\left(c_{j}\left(1-\alpha_{n}\right)^{j}\right)^{2}\right) \exp \left(\lambda\left(c_{j-1}-\alpha_{n}\right) X_{j-1}\right) .
\end{aligned}
$$


We can then apply to the expectation in (81), i.e.

$$
\begin{aligned}
\mathbb{E}_{n} & {\left[\prod_{j=1}^{m-1} \exp \left(\lambda \alpha_{n} X_{j}\right)\right]=\mathbb{E}_{n}\left[\prod_{j=1}^{m-2} \exp \left(\lambda \alpha_{n} X_{j}\right) \exp \left(\lambda c_{m-1} X_{m-1}\right)\right] } \\
& =\mathbb{E}_{n}\left[\prod_{j=1}^{m-2} \exp \left(\lambda \alpha_{n} X_{j}\right) \mathbb{E}_{n}\left[\exp \left(\lambda c_{m-1} X_{m-1}\right) \mid X^{m-2}\right]\right] \\
& \leq \mathbb{E}_{n}\left[\prod_{j=1}^{m-2} \exp \left(\lambda \alpha_{n} X_{j}\right) \exp \left(\lambda\left(c_{m-2}-\alpha_{n}\right) X_{m-2}\right)\right] \exp \left(\frac{\lambda^{2}}{8}\left(c_{m-1}\left(1-\alpha_{n}\right)^{m-1}\right)^{2}\right)
\end{aligned}
$$

and so applying recursively eventually gives

$$
\mathbb{E}_{n}\left[\prod_{j=1}^{m-1} \exp \left(\lambda \alpha_{n} X_{j}\right)\right] \leq \exp \left(\lambda \mathbb{E}_{n}\left[\alpha_{n} \sum_{i=1}^{m-1} X_{i}\right]+\frac{\lambda^{2}}{8} \sum_{j=1}^{m-1}\left(c_{j}\left(1-\alpha_{n}\right)^{j}\right)^{2}\right),
$$

where we have also used $X_{0}=1$ and (83). Substituting into (81),

$$
\mathbb{P}_{n}\left[\alpha_{n} \sum_{j=1}^{m-1} X_{j}>\frac{\epsilon}{2}\right] \leq \min _{\lambda>0} \exp \left(-\lambda\left(\frac{\epsilon}{2}-\mathbb{E}_{n}\left[\alpha_{n} \sum_{i=1}^{m-1} X_{i}\right]\right)+\frac{\lambda^{2}}{8} \sum_{j=1}^{m-1}\left(c_{j}\left(1-\alpha_{n}\right)^{j}\right)^{2}\right) .
$$

It is straightforward to show the global minimizer of (84) is

$$
\lambda^{*}=\frac{4\left(\frac{\epsilon}{2}-\mathbb{E}_{n}\left[\alpha_{n} \sum_{i=1}^{m-1} X_{i}\right]\right)}{\sum_{j=1}^{m-1}\left(c_{j}\left(1-\alpha_{n}\right)^{j}\right)^{2}}
$$

which is positive when $\mathbb{E}_{n}\left[\alpha_{n} \sum_{i=1}^{m-1} X_{i}\right]<\frac{\epsilon}{2}$. Plugging into (84),

$$
\mathbb{P}_{n}\left[\alpha_{n} \sum_{j=1}^{m-1} X_{j}>\frac{\epsilon}{2}\right] \leq \exp \left(-\frac{2\left(\frac{\epsilon}{2}-\mathbb{E}_{n}\left[\alpha_{n} \sum_{i=1}^{m-1} X_{i}\right]\right)^{2}}{\sum_{j=1}^{m-1}\left(c_{j}\left(1-\alpha_{n}\right)^{j}\right)^{2}}\right) .
$$

We now derive bounds for the denominator and numerator in the exponential in (85). To (coarsely) approximate the denominator,

$$
\begin{aligned}
& c_{j}<\frac{\alpha_{n}}{1-\left(1-\alpha_{n}\right) \hat{p}_{n}}<\frac{\alpha_{n}}{1-\hat{p}_{n}}, \quad \sum_{j=1}^{m-1}\left(1-\alpha_{n}\right)^{2 j}<\sum_{j=0}^{\infty}\left(1-\alpha_{n}\right)^{j}=\frac{1}{\alpha_{n}} \\
& \Rightarrow \sum_{j=1}^{m-1}\left(c_{j}\left(1-\alpha_{n}\right)^{j}\right)^{2}<\frac{\alpha_{n}}{\left(1-\hat{p}_{n}\right)^{2}}<\frac{\alpha_{n}}{\left(1-p-n^{-\gamma}\right)^{2}},
\end{aligned}
$$

where the final inequality holds assuming $\Omega_{n}$ and $n$ is sufficiently large (so that $p+n^{-\gamma}<1$ ). For the numerator, first observe that, when $\Omega_{n}$ holds, we have

$$
\mathbb{E}_{n}\left[\alpha_{n} \sum_{i=1}^{m-1} X_{i}\right]=\frac{\alpha_{n}\left(\left(1-\alpha_{n}\right) \hat{p}_{n}-\left(\left(1-\alpha_{n}\right) \hat{p}_{n}\right)^{m}\right)}{1-\left(1-\alpha_{n}\right) \hat{p}_{n}}<\frac{\alpha_{n}}{1-\left(p+n^{-\gamma}\right)},
$$

and so $\mathbb{E}_{n}\left[\alpha_{n} \sum_{i=1}^{m-1} X_{i}\right]<\epsilon / 2$ for $n$ sufficiently large (as required), assuming $\alpha_{n} \rightarrow 0$ as $n \rightarrow \infty$. Therefore, when $\Omega_{n}$ holds, $\alpha_{n} \rightarrow 0$, and $n$ is large,

$$
\left(\frac{\epsilon}{2}-\mathbb{E}_{n}\left[\alpha_{n} \sum_{i=1}^{m-1} X_{i}\right]\right)^{2}>\left(\frac{\epsilon}{2}-\frac{\alpha_{n}}{1-\left(p+n^{-\gamma}\right)}\right)^{2}=\frac{\epsilon^{2}}{4}-\frac{\alpha_{n}}{1-\left(p+n^{-\gamma}\right)}\left(\epsilon-\frac{\alpha_{n}}{1-\left(p+n^{-\gamma}\right)}\right) \text {. }
$$


Thus, under these assumptions, (86) and (87) give

$$
\frac{2\left(\frac{\epsilon}{2}-\mathbb{E}_{n}\left[\alpha_{n} \sum_{i=1}^{m-1} X_{i}\right]\right)^{2}}{\sum_{j=1}^{m-1}\left(c_{j}\left(1-\alpha_{n}\right)^{j}\right)^{2}}>\frac{\left(1-p-n^{-\gamma}\right)^{2} \epsilon^{2}}{2 \alpha_{n}}-2\left(1-p-n^{-\gamma}\right)\left(\epsilon-\frac{\alpha_{n}}{1-\left(p+n^{-\gamma}\right)}\right) .
$$

To summarize, we have shown that for $n$ sufficiently large, assuming $\alpha_{n} \rightarrow 0$ and $\Omega_{n}$ holds,

$$
\begin{aligned}
\mathbb{P}_{n}\left[\alpha_{n} \sum_{j=1}^{m-1} X_{j}>\frac{\epsilon}{2}\right] & \leq \exp \left(-\frac{\left(1-p-n^{-\gamma}\right)^{2} \epsilon^{2}}{2 \alpha_{n}}\right) \exp \left(2\left(1-p-n^{-\gamma}\right)\left(\epsilon-\frac{\alpha_{n}}{1-\left(p+n^{-\gamma}\right)}\right)\right) \\
& =O\left(\exp \left(-\frac{((1-p) \epsilon)^{2}}{2 \alpha_{n}}\right)\right)
\end{aligned}
$$

where the equality holds because the second exponential term is $O(1)$ for $p \in(0,1)$. Finally, we combine (77), (78), (79), (80), and (88) to obtain

$$
\mathbb{P}\left[\alpha_{n} \sum_{j=1}^{m} X_{j}+X_{m}>\epsilon\right]=O\left(n^{-\delta}+p^{m}+e^{-((1-p) \epsilon)^{2} /\left(2 \alpha_{n}\right)}\right),
$$

which completes the proof.

\section{D.6 Simultaneous construction of graph and tree}

For the proofs of Lemmas 4 and 5, we use Algorithm 9, which simultaneously constructs a graph and a tree. Algorithm 9 uses similar notation as Algorithms 5 and 6 in Appendix A.2. However, there are some differences, which we explain first.

- In Algorithm 5, we chose $s \sim V_{n}$ uniformly, which is the standard DCM construction. In Algorithm 9, we instead choose $s \sim V_{n} \backslash K_{n}$ uniformly. This is because in the statement of Lemma 4 involves $\mu_{s}^{(m)}\left(V_{n} \backslash K_{n}\right)$, conditioned on $U_{s}=1$ (i.e. $\left.s \in V_{n} \backslash K_{n}\right)$; similarly, the statement of Lemma 5 involves $\left\{\tau_{G} \leq m\right\}$, conditioned on $U_{s}=1$.

- Algorithm 9 uses a function $\Phi: V_{n} \rightarrow \mathcal{U}$, where $\mathcal{U}=\cup_{j=0}^{\infty} \mathbb{N}^{j}$ and $\mathbb{N}^{0}=\{\phi\}$ by convention. The function $\Phi$ will be used to map nodes in the graph (which have labels in the set $V_{n}$, as in Algorithm 5) to nodes in the tree (which have labels in the set $\mathcal{U}$, as in Algorithm 6).

- The variable $\tau_{S}$ in Algorithm 9 denotes the first iteration at which events that break the coupling occur (analogous to $\tau_{G}$ in Algorithm 5). Once these events occur, the simultaneous construction terminates, and the graph and tree constructions are continued separately using Algorithms 5 and 6, respectively.

For illustrative purposes, we include an example of the simultaneous construction in Figure 12. The basic idea is as follows. Whenever a new node is added to the graph, (which occurs when outstub $\left(v^{\prime}, j\right)$ is paired with an instub belonging to $v \in V_{n}$ s.t. $g(v)=A$ ) a new offspring (with the same attributes as $v$ ) is added to the tree, and a map between the graph node and tree offspring is defined. In particular, Figure 12 has the following mapping:

$$
\begin{gathered}
\Phi(s)=\phi, \quad \Phi(1)=(1), \quad \Phi(2)=(2), \quad \Phi(3)=(1,2), \\
\Phi(4)=(1,3), \quad \Phi(5)=(2,1), \quad \Phi(6)=(2,3), \quad \Phi(7)=(2,4) .
\end{gathered}
$$

If an edge is added between two nodes already in the graph (which occurs when outstub $\left(v^{\prime}, j\right)$ is paired with an instub belonging to $v \in V_{n}$ s.t. $g(v) \in\{B, C, D\}$ ), a new offspring with the same attributes as $v$ is added to the tree. This is illustrated in Figure 12 by the following examples:

- Node 1 in the graph adds an edge to itself; offspring $(1,1)$ in the tree has the attributes of 1

- Node 1 in the graph adds an edge to 2 ; offspring $(1,4)$ in the tree has the attributes of 2 


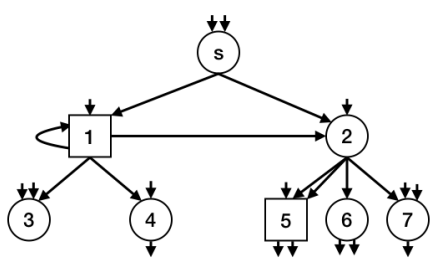

(a) Graph

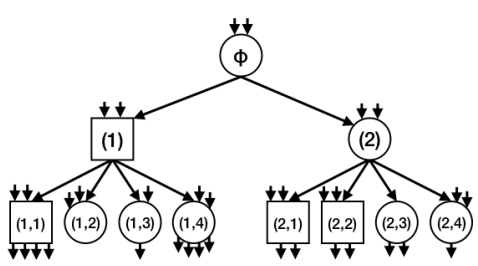

(b) Tree

Fig. 12. Simultaneous construction example

- Node 2 in the graph adds a multi-edge to 5 ; offspring $(2,2)$ in the tree has the attributes of 5 These offspring can be thought of as copies of nodes already in the tree: $(1,1),(1,4)$, and $(2,2)$ are copies of (1), (2), and (2,1), respectively. Furthermore, note that for $\mathbf{i} \in\{(1,1),(1,4),(2,2)\}$, $\Phi^{-1}(\mathbf{i})=\emptyset$. In other words, copies of nodes in the tree do not map back to nodes in the graph. This implies that we may have more nodes in the tree than in the graph. For this reason, after pairing all outstubs belonging to all $v^{\prime} \in A_{m-1}$ (which map to nodes in the tree), we must separately add offspring to nodes $\mathbf{i} \in \hat{A}_{m-1}$ s.t. $\Phi^{-1}(\mathbf{i})=\emptyset$ (which do not map to nodes in the tree). This is done in Lines 30-34 in Algorithm 9.

\section{E PROOF OF PROPOSITION 1}

First, note that $\forall n \in \mathbb{N}, \forall l \in \mathbb{N}$, and $\forall l^{\prime} \leq l$, we have (a.s.)

$$
\pi_{s}\left(V_{n, s}(l)\right) \geq \alpha_{n} \sum_{j=0}^{l}\left(1-\alpha_{n}\right)^{j}\left(e_{s}^{\mathrm{T}} P^{j} 1_{n}\right)=\alpha_{n} \sum_{j=0}^{l}\left(1-\alpha_{n}\right)^{j}=1-\left(1-\alpha_{n}\right)^{l+1} \geq 1-\left(1-\alpha_{n}\right)^{l^{\prime}},
$$

where the first inequality follows from (4) and by definition of $V_{n, s}(l)$, and the first equality holds since $P^{j}$ is row stochastic (the remaining steps are simple manipulations). Therefore, when $\alpha_{n}=\frac{\rho \log (1 / \tau) \log \zeta}{\log n}$, we can define $c=\rho \log \zeta$ and use (89) to write

$$
\liminf _{n \rightarrow \infty} \pi_{s}\left(V_{n, s}\left(\left[\frac{\log (1 / \tau)}{\alpha_{n}}\right]\right)\right) \geq 1-\lim _{n \rightarrow \infty}\left(1+\frac{\log (\tau)}{\log (n) / c}\right)^{\log (n) / c}=1-\tau \text { a.s. },
$$

which is the desired bound. If instead $\alpha_{n}=\alpha$ is a constant, we have more simply

$$
\liminf _{n \rightarrow \infty} \pi_{s}\left(V_{n, s}\left(\left[\frac{\log (1 / \tau)}{\log (1 /(1-\alpha))}\right]\right)\right) \geq 1-(1-\alpha)^{\frac{\log (1 / \tau)}{\log (1 /(1-\alpha))}}=1-(1-\alpha)^{\log _{(1-\alpha)}(\tau)}=1-\tau \text { a.s., }
$$

which is the other desired bound. Next, to bound the expected size of $V_{n, s}(l)$, we use the analysis of Appendix D.4.2. First, for $l \in \mathbb{N}$, the argument preceding (72) in Appendix D.4.2 implies

$$
\left|V_{n, s}(l)\right| \leq \sum_{j=0}^{l} \hat{Z}_{j}
$$

where $\hat{Z}_{j}$ is defined in (68). Furthermore, by (74) in Appendix D.4.2, we have for $j \in \mathbb{N}$,

$$
\mathbb{E}\left[\hat{Z}_{j} \mid \Omega_{n}\right]=O\left(\zeta^{j-1}\right),
$$

while $\hat{Z}_{0}=1$ by definition. Combining the previous two equations gives for $l \in \mathbb{N}$,

$$
\mathbb{E}\left[V_{n, s}(l) \mid \Omega_{n}\right]=O\left(1+\sum_{j=0}^{l-1} \zeta^{j}\right)=O\left(\zeta^{l}\right) \text {. }
$$




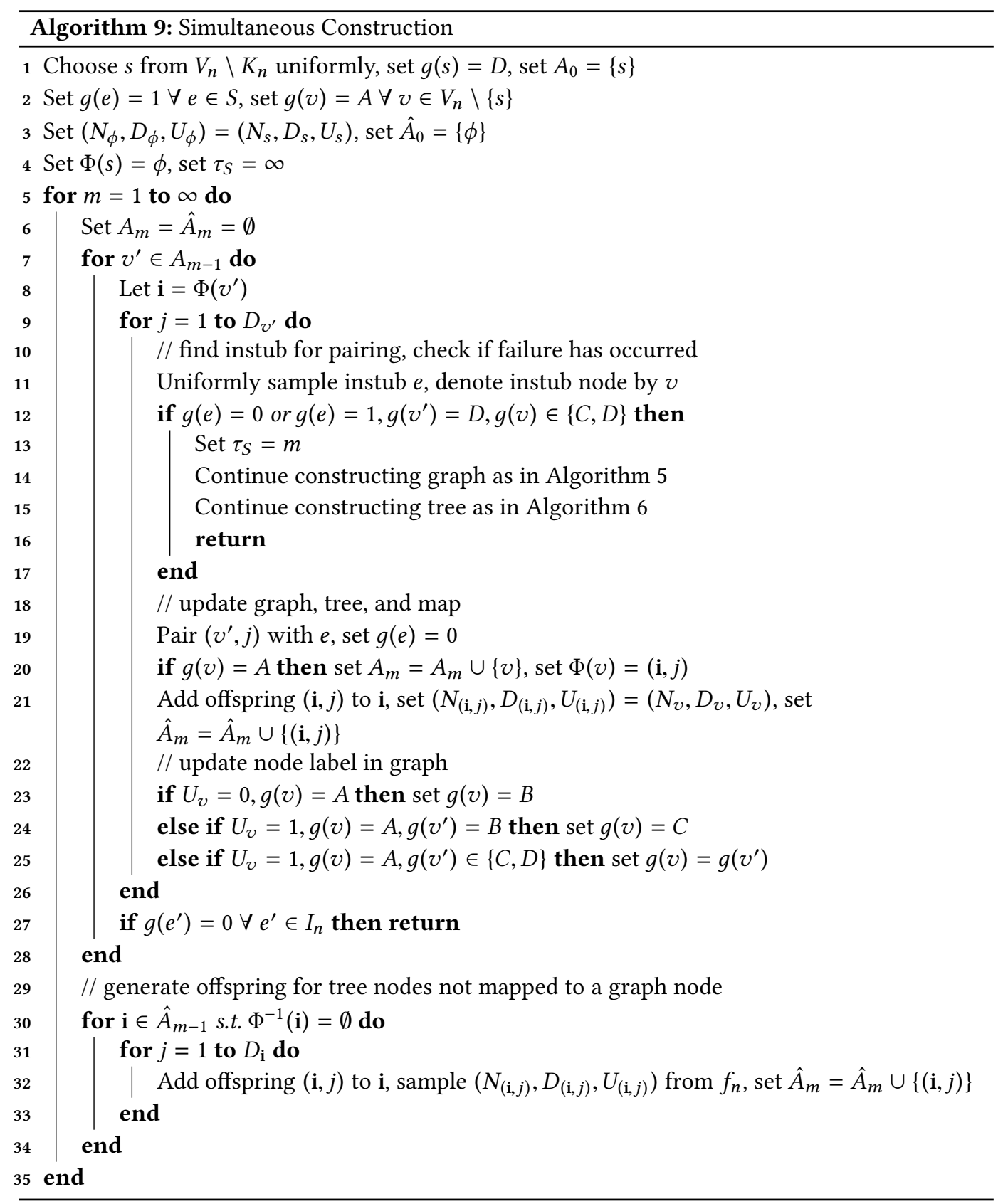

Therefore, when $\alpha_{n}=\frac{\rho \log (1 / \tau) \log \zeta}{\log n}$, we have

$$
\mathbb{E}\left[\mid V_{n, s}\left(\left[\frac{\log (1 / \tau)}{\alpha_{n}}\right]\right) \| \Omega_{n}\right]=O\left(\zeta^{\log (1 / \tau) / \alpha_{n}}\right)=O\left(\zeta^{\log _{\zeta}\left(n^{1 / \rho}\right)}\right)=O\left(n^{1 / \rho}\right) .
$$


Similarly, if $\alpha_{n}=\alpha$ is a constant,

$$
\mathbb{E}\left[\mid V_{n, s}\left(\left[\frac{\log (1 / \tau)}{\log (1 /(1-\alpha))}\right]\right) \| \Omega_{n}\right]=O\left(\zeta^{\log (1 / \tau) / \log (1 /(1-\alpha))}\right),
$$

which is $O(1)$.

\section{F PROOF OF THEOREM 1}

First, we observe

$$
\begin{aligned}
\mathbb{E}\left[\Delta\left(K_{n}, \epsilon\right)\right] & =\mathbb{E}\left[\left|K_{n}\right|+\mid\left\{v \in V_{n} \backslash K_{n}: B_{v}\left(K_{n}, \epsilon\right) \text { holds }\right\} \mid\right] \\
& =O\left(n^{\kappa}\right)+\sum_{v \in V_{n}} \mathbb{E}\left[1\left(B_{v}\left(K_{n}, \epsilon\right), U_{v}=1\right)\right]=O\left(n^{\kappa}\right)+n \mathbb{E}\left[1\left(B_{s}\left(K_{n}, \epsilon\right), U_{s}=1\right)\right] \\
& \leq O\left(n^{\kappa}\right)+n \mathbb{P}\left[B_{s}\left(K_{n}, \epsilon\right) \mid U_{s}=1\right]=O\left(n^{\kappa}\right)+n O\left(n^{-c(\epsilon)}\right)=O\left(n^{\max \{\kappa, 1-c(\epsilon)\}}\right),
\end{aligned}
$$

where the successive steps hold by definition of $\Delta\left(K_{n}, \epsilon\right)$, by Assumption 2 , since $1\left(B_{v}\left(K_{n}, \epsilon\right), U_{v}=\right.$ 1) are identically distributed before the degree sequence is realized, since $\mathbb{P}\left[U_{v}=1\right] \leq 1$, and by Lemma 1, respectively. Hence, by Markov's inequality,

$$
\mathbb{P}\left[\Delta\left(K_{n}, \epsilon\right) \geq C n^{\bar{c}}\right] \leq \frac{\mathbb{E}\left[\Delta\left(K_{n}, \epsilon\right)\right]}{C n^{\bar{c}}}=O\left(n^{\max \{\kappa, 1-c(\epsilon)\}-\bar{c}}\right) .
$$

\section{G PROOF OF THEOREM 2}

\section{G.1 Analysis of subroutines of Algorithm 1}

We begin with analyses of Approx-PageRank and Approx-Contributions. Namely, Lemma 12 gives accuracy and complexity guarantees for Approx-PageRank, while Lemma 13 and Corollary 1 provide guarantees for Approx-Contributions. We note that these results are essentially restatements of those found in [3,4]; we have included the arguments because they need to be slightly modified (as mentioned in the main text), and to state them using our notation.

Lemma 12. For any realization of $G_{n}$, any $v \in V_{n}$, and any $\epsilon_{1} \in(0,1)$, Approx-PageRank $\left(v, \epsilon_{1}\right)$ has complexity $O\left(L_{n} /\left(\alpha_{n} \epsilon_{1}\right)\right)$, and the output $\hat{\pi}_{v}$ satisfies $\left\|\pi_{v}-\hat{\pi}_{v}\right\|_{1} \leq \epsilon_{1}, \hat{\pi}_{v}(u) \leq \pi_{v}(u) \forall u \in V_{n}$.

Proof. We first claim that for each $u \in V_{n}$ and at each iteration of Approx-PageRank,

$$
\pi_{v}(u)=\hat{\pi}_{v}(u)+\sum_{w \in V_{n}} r_{v}(w) \pi_{w}(u) .
$$

To prove (92), first note that since $\hat{\pi}_{v}$ and $r_{v}$ are initialized to $0_{n}$ and $e_{v}$, respectively, it holds trivially at the beginning of the algorithm. Now assume (92) holds before $\hat{\pi}_{v}$ and $r_{v}$ are updated at some iteration. Then after the update, we will have

$$
\begin{aligned}
& \left(\hat{\pi}_{v}(u)+\alpha_{n} r_{v}\left(v^{*}\right) 1\left(u=v^{*}\right)\right)+\sum_{w \in V_{n}}\left(r_{v}(w) 1\left(w \neq v^{*}\right)+\left(1-\alpha_{n}\right) r_{v}\left(v^{*}\right) P\left(v^{*}, w\right)\right) \pi_{w}(u) \\
& =\left(\hat{\pi}_{v}(u)+\alpha_{n} r_{v}\left(v^{*}\right) 1\left(u=v^{*}\right)\right)+\sum_{w \in V_{n} \backslash\left\{v^{*}\right\}} r_{v}(w) \pi_{w}(u)+r_{v}\left(v^{*}\right)\left(1-\alpha_{n}\right) \sum_{w \in V_{n}} P\left(v^{*}, w\right) \pi_{w}(u) \\
& =\left(\hat{\pi}_{v}(u)+\alpha_{n} r_{v}\left(v^{*}\right) 1\left(u=v^{*}\right)\right)+\sum_{w \in V_{n} \backslash\left\{v^{*}\right\}} r_{v}(w) \pi_{w}(u)+r_{v}\left(v^{*}\right)\left(\pi_{v^{*}}(u)-\alpha_{n} 1\left(u=v^{*}\right)\right) \\
& =\hat{\pi}_{v}(u)+\sum_{w \in V_{n}} r_{v}(w) \pi_{w}(u)=\pi_{v}(u),
\end{aligned}
$$


where the final equality uses the assumption that (92) holds before the update, and where the second equality holds since by (4),

$$
\begin{aligned}
\pi_{v^{*}} & =\alpha_{n} e_{v^{*}}^{\top}+\alpha_{n} e_{v^{*}}^{\top}\left(1-\alpha_{n}\right) P \sum_{i=0}^{\infty}\left(1-\alpha_{n}\right)^{i} P^{i} \\
& =\alpha_{n} e_{v^{*}}^{\top}+\left(1-\alpha_{n}\right) \sum_{w \in V_{n}} P\left(v^{*}, w\right) \alpha_{n} e_{w}^{\top} \sum_{i=0}^{\infty}\left(1-\alpha_{n}\right)^{i} P^{i}=\alpha_{n} e_{v^{*}}^{\top}+\left(1-\alpha_{n}\right) \sum_{w \in V_{n}} P\left(v^{*}, w\right) \pi_{w} .
\end{aligned}
$$

Next, observe that $\pi_{w}(u) \geq 0 \forall w, u \in V_{n}$ by definition; further, $r_{v}(w) \geq 0 \forall w \in V_{n}$ for the duration of the algorithm. Together with (92), this implies $\hat{\pi}_{v}(u) \leq \pi_{v}(u) \forall u \in V_{n}$, as claimed.

To show $\left\|\pi_{v}-\hat{\pi}_{v}\right\|_{1} \leq \epsilon_{1}$ at termination, observe

$$
\begin{aligned}
\left\|\pi-\hat{\pi}_{v}\right\|_{1} & =\sum_{u \in V_{n}}\left(\pi_{v}(u)-\hat{\pi}_{v}(u)\right)=\sum_{u \in V_{n}} \sum_{w \in V_{n}} r_{v}(w) \pi_{w}(u)=\sum_{w \in V_{n}} r_{v}(w) \sum_{u \in V_{n}} \pi_{w}(u) \\
& =\sum_{w \in V_{n}} r_{v}(w)=\sum_{w \in V_{n}} \frac{r_{v}(w)}{D_{w}} D_{w} \leq \frac{\epsilon_{1}}{L_{n}} \sum_{w \in V_{n}} D_{w}=\epsilon_{1},
\end{aligned}
$$

where the first equality holds by $\hat{\pi}_{v}(u) \leq \pi_{v}(u)$, the second holds by (92), and the fourth uses the fact that $\pi_{w}$ sums to 1 (the others are immediate); the inequality holds at termination of the algorithm via the terminating condition of the while loop.

For the complexity guarantee, let $i^{*}$ denote the iteration at which the algorithm terminates, and let $v_{i}$ be the node chosen as $v^{*}$ during the $i$-th iteration. Then it is readily verified that $\left\|r_{v}\right\|_{1}$ decreases by $\alpha_{n} r_{v}\left(v_{i}\right)=\alpha_{n} \frac{r_{v}\left(v_{i}\right)}{D_{v_{i}}} D_{v_{i}} \geq \alpha_{n} \frac{\epsilon_{1}}{L_{n}} D_{v_{i}}$ at the $i$-th iteration. Hence, because $\left\|r_{v}\right\|_{1}=1$ initially and is bounded below by zero,

$$
1 \geq \sum_{i=1}^{i^{*}} \alpha_{n} r_{v}\left(v_{i}\right) \geq \alpha_{n} \frac{\epsilon_{1}}{L_{n}} \sum_{i=1}^{i^{*}} D_{v_{i}} \Rightarrow \sum_{i=1}^{i^{*}} D_{v_{i}} \leq \frac{L_{n}}{\alpha_{n} \epsilon_{1}} .
$$

On the other hand, at most $D_{v_{i}}$ elements of the $r_{v}$ vector and one element of the $\hat{\pi}_{v}$ vectors are updated at iteration $i$, so the complexity of the algorithm scales with $\sum_{i=1}^{i^{*}} D_{v_{i}}$. Hence, the complexity is bounded by $L_{n} /\left(\alpha_{n} \epsilon_{1}\right)$, as claimed.

LEMMA 13. For any realization of $G_{n}, v \in V_{n}$, and $\epsilon_{2} \in(0,1)$, Approx-Contributions $\left(v, \epsilon_{2}\right)$ has complexity

$$
O\left(\frac{1}{\epsilon_{2}} \sum_{u \in V} \mu_{u}(v) N_{u}\right)
$$

and the output $\left\{\hat{\mu}_{u}(v)\right\}_{u \in V_{n}}$ satisfies $\left|\mu_{u}(v)-\hat{\mu}_{u}(v)\right| \leq \epsilon_{2} / \alpha_{n}$ and $\hat{\mu}_{u}(v) \leq \mu_{u}(v) \forall u \in V_{n}$, where $\mu_{u}(v)$ is the $v$-th element of the vector $\mu_{u}$ given by (54) in Appendix D.2.

Proof. We begin with a claim analogous to (92); namely, that for each $u \in V_{n}$ and at each iteration of Approx-Contributions,

$$
\mu_{u}(v)=\hat{\mu}_{u}(v)+\sum_{w \in V_{n}} \mu_{u}(w) r_{v}(w)
$$


As for (92), (93) is immediate at the start of the algorithm, and if it holds before $\left\{\hat{\mu}_{u}(v)\right\}_{u \in V_{n}}$ and $r_{v}$ are updated, we have

$$
\begin{aligned}
& \left(\hat{\mu}_{u}(v)+r_{v}\left(v^{*}\right) 1\left(u=v^{*}\right)\right)+\sum_{w \in V_{n}} \mu_{u}(w)\left(r_{v}(w) 1\left(w \neq v^{*}\right)+\left(1-\alpha_{n}\right) r_{v}\left(v^{*}\right) \tilde{P}\left(w, v^{*}\right)\right) \\
& =\left(\hat{\mu}_{u}(v)+r_{v}\left(v^{*}\right) 1\left(u=v^{*}\right)\right)+\sum_{w \in V_{n} \backslash\left\{v^{*}\right\}} \mu_{u}(w) r_{v}(w)+r_{v}\left(v^{*}\right)\left(1-\alpha_{n}\right) \sum_{w \in V_{n}} \mu_{u}(w) \tilde{P}\left(w, v^{*}\right) \\
& =\left(\hat{\mu}_{u}(v)+r_{v}\left(v^{*}\right) 1\left(u=v^{*}\right)\right)+\sum_{w \in V_{n} \backslash\left\{v^{*}\right\}} \mu_{u}(w) r_{v}(w)+r_{v}\left(v^{*}\right)\left(\mu_{u}\left(v^{*}\right)-1\left(u=v^{*}\right)\right) \\
& =\hat{\mu}_{u}(v)+\sum_{w \in V_{n}} \mu_{u}(w) r_{v}(w)=\mu_{u}(v),
\end{aligned}
$$

where the final step is because (93) holds before the update by assumption, and the second equality holds since by (54),

$$
\begin{aligned}
\mu_{u}\left(v^{*}\right) & =e_{u}^{T} \sum_{i=0}^{\infty}\left(1-\alpha_{n}\right)^{i} \tilde{P}^{i} e_{v^{*}}=1\left(u=v^{*}\right)+\left(1-\alpha_{n}\right) e_{u}^{T} \sum_{i=0}^{\infty}\left(1-\alpha_{n}\right)^{i} \tilde{P}^{i} \tilde{P} e_{v^{*}} \\
& =1\left(u=v^{*}\right)+\left(1-\alpha_{n}\right) e_{u}^{T} \sum_{i=0}^{\infty}\left(1-\alpha_{n}\right)^{i} \tilde{P}^{i} \sum_{w \in V_{n}} \tilde{P}\left(w, v^{*}\right) e_{w} \\
& =1\left(u=v^{*}\right)+\left(1-\alpha_{n}\right) \sum_{w \in V_{n}} e_{u}^{T} \sum_{i=0}^{\infty}\left(1-\alpha_{n}\right)^{i} \tilde{P}^{i} e_{w} \tilde{P}\left(w, v^{*}\right) \\
& =1\left(u=v^{*}\right)+\left(1-\alpha_{n}\right) \sum_{w \in V_{n}} \mu_{u}(w) \tilde{P}\left(w, v^{*}\right) .
\end{aligned}
$$

From (93), the fact that $\mu_{u}(w) \geq 0 \forall u, w \in V_{n}$ by definition, and the fact that $r_{v}(w) \geq 0 \forall w \in V_{n}$ for the duration of the algorithm, we immediately obtain $\hat{\mu}_{u}(v) \leq \mu_{u}(v) \forall u \in V_{n}$, as claimed.

For the accuracy guarantee, note that at termination, we have

$$
\mu_{u}(v)-\hat{\mu}_{u}(v)=\sum_{w \in V_{n}} \mu_{u}(w) r_{v}(w) \leq \epsilon_{2} \sum_{w \in V_{n}} \mu_{u}(w) \leq \epsilon_{2} / \alpha_{n},
$$

where the equality holds by (93), the first inequality is by the terminating condition of the while loop, and the final inequality holds by definition of $\mu_{u}$ and the fact that $\tilde{P}$ is nonnegative with row sums bounded by 1 , together which imply

$$
\sum_{w \in V_{n}} \mu_{u}(w)=\sum_{i=0}^{\infty}\left(1-\alpha_{n}\right)^{i} e_{u}^{\top} \tilde{P}^{i} 1_{n} \leq \sum_{i=0}^{\infty}\left(1-\alpha_{n}\right)^{i}=\frac{1}{\alpha_{n}} .
$$

For the complexity guarantee, first note that $\hat{\mu}_{u}(v)$ increases by $r_{v}(u)>\epsilon_{2}$ at each iteration for which $v^{*}=u$; hence, because $\hat{\mu}_{u}(v) \leq \mu_{u}(v)$, we can have $v^{*}=u$ for at most $\mu_{u}(v) / \epsilon_{2}$ iterations. Furthermore, the complexity of each such iteration scales with $N_{u}$ (as in the argument in the proof of Lemma 12). Hence, the overall complexity can be bounded by $\sum_{u \in V_{n}} \mu_{u}(v) N_{u} / \epsilon_{2}$.

COROLlaRy 1. For any realization of $G_{n}$ and $\epsilon_{2} \in(0,1)$, running Approx-Contributions $\left(k, \epsilon_{2}\right)$ for each $k \in K_{n}$ produces output $\hat{\mu}_{v}(k)$ satisfying $\left|\mu_{v}(k)-\hat{\mu}_{v}(k)\right| \leq \epsilon_{2} / \alpha_{n}$ and $\hat{\mu}_{v}(k) \leq \mu_{v}(k)$ for each $v \in V_{n}, k \in K_{n}$; additionally, if $L_{n}=O(n)$ and $\epsilon_{2}$ depends on $n$, the complexity is $O\left(n / \epsilon_{2}\right)$. 
Proof. The accuracy guarantee follows from Lemma 13. Furthermore, by Lemma 13, we can bound the complexity as

$$
\frac{1}{\epsilon_{2}} \sum_{k \in K_{n}} \sum_{u \in V_{n}} \mu_{u}(k) N_{u}=\frac{1}{\epsilon_{2}} \sum_{u \in V_{n}} N_{u} \mu_{u}\left(K_{n}\right) .
$$

Using (56), it is straightforward to show

$$
\mu_{u}\left(K_{n}\right)=\frac{\tilde{\pi}_{u}\left(K_{n}\right)}{\alpha_{n}+\left(1-\alpha_{n}\right) \tilde{\pi}_{u}\left(K_{n}\right)} \leq 1 \forall u \in V_{n},
$$

where we have also used $\tilde{\pi}_{u}\left(K_{n}\right) \leq 1$. Combining the previous two equations gives complexity $L_{n} / \epsilon_{2}$, from which the corollary follows by assumption $L_{n}=O(n)$.

\section{G.2 Proof of Theorem 2}

With Lemmas 12 and 13 and Corollary 1 in place, we turn to the proof of Theorem 2. We begin with the complexity guarantee. For this, we will proceed through each of the six computations undertaken by Algorithm 1 and bound the complexity of each.

First, let $C_{A l g 1}^{(1)}$ denote the complexity of running Approx-Page- Rank $\left(k, \epsilon_{1}\right) \forall k \in K_{n}$. By Lemma 12, $C_{A l g 1}^{(1)}=O\left(\left|K_{n}\right| L_{n} /\left(\alpha_{n} \epsilon_{1}\right)\right)$. Since $L_{n}=O(n)$ when $\Omega_{n}$ holds, $\alpha_{n}=\Theta\left(\frac{1}{\log n}\right)$, and $\epsilon_{1}$ is constant,

$$
\mathbb{E}\left[C_{A l g 1}^{(1)} \mid \Omega_{n}\right]=O\left(\mathbb{E}\left[\left|K_{n}\right| \mid \Omega_{n}\right] n \log n\right) .
$$

Next, let $C_{A l g 1}^{(2)}$ denote the complexity of running Approx-Cont-ributions $\left(k, \epsilon_{2}\right)$ for every $k \in K_{n}$. By Corollary 1 , this has complexity $O\left(n / \epsilon_{2}\right)$ when $L_{n}=O(n)$, which occurs when $\Omega_{n}$ holds. Since also $\epsilon_{2}=\frac{\alpha_{n}^{2} g_{n}(\epsilon / 4)}{2\left|K_{n}\right|}$, this is $O\left(\mathbb{E}\left[\left|K_{n}\right| \mid \Omega_{n}\right] n \alpha_{n}^{2} g_{n}(\epsilon / 4)\right)$ in expectation when $\Omega_{n}$ holds. Furthermore, since $\alpha_{n} \rightarrow 0$ as $n \rightarrow \infty$ and $\epsilon \in(0,1)$ is constant, we have $1-2(\epsilon / 4)<1-\alpha_{n}-(\epsilon / 4)$ and $\epsilon / 4>\alpha_{n}\left(2-\alpha_{n}-(\epsilon / 4)\right)$ for large enough $n$; for such $n$,

$$
\frac{\alpha_{n}(1-2(\epsilon / 4))}{2(\epsilon / 4)}<\frac{\alpha_{n}\left(1-\alpha_{n}-(\epsilon / 4)\right)}{(\epsilon / 4)+\left(2-\alpha_{n}-(\epsilon / 4)\right)}=g_{n}(\epsilon / 4)
$$

It is also immediate that $g_{n}(\epsilon / 4) \leq \frac{\alpha_{n}}{\epsilon / 4}$. Taken together,

$$
g_{n}(\epsilon / 4) \in\left(\frac{\alpha_{n}(1-2(\epsilon / 4))}{2(\epsilon / 4)}, \frac{\alpha_{n}}{\epsilon / 4}\right) \Rightarrow g_{n}(\epsilon / 4)=\Theta\left(\alpha_{n}\right) .
$$

Hence, with $\alpha_{n}=\Theta\left(\frac{1}{\log n}\right)$, we conclude

$$
\mathbb{E}\left[C_{A l g 1}^{(2)} \mid \Omega_{n}\right]=O\left(\mathbb{E}\left[\mid K_{n} \| \Omega_{n}\right] n(\log n)^{3}\right) .
$$

Next, let $C_{A l g 1}^{(3)}$ denote the complexity of constructing $\hat{\pi}_{k}^{l}$. We claim that this can be done while running Approx-PageRank $\left(k, \epsilon_{1}\right)$ without increasing the order of the Approx-PageRank complexity. This argument is based on the fact that Approx-PageRank $\left(k, \epsilon_{1}\right)$ essentially completes a breadthfirst-search out of $k$. First, we can set $V_{n, k}(0)=\{k\}$ at the initial iteration, and each time a new node is encountered, we can add it to $V_{n, k}(j+1)$ if its previously-encountered incoming neighbor belongs to $V_{n, k}(j)$ for some $j$. (By encountering a new node $u$, we mean incrementing $r_{v}(u)$ for the first time; note that $r_{v}(u)$ is incremented only if $u$ 's incoming neighbor $w$ is chosen as $v^{*}$, which in turn occurs only if $r_{v}(w)$ is nonzero, which means $w$ has been previously encountered.) Next, observe that $\hat{\pi}_{k}(u)$ is not updated until $u$ is first encountered, at which point we can check if $u \in V_{n, k}(j)$ for some $j \leq l$; if it is, $\hat{\pi}_{k}^{l}(u)$ can be updated each time $\hat{\pi}_{k}(u)$ is updated. Adding nodes to $V_{n, k}(j+1)$ has complexity that scales with that of updating $r_{v}$, while updating $\hat{\pi}_{k}^{l}$ has complexity that scales with 
that of updating $\hat{\pi}_{k}$. Hence, constructing $\hat{\pi}_{k}^{l}$ has complexity bounded by the Approx-PageRank complexity. In other words, we have

$$
\mathbb{E}\left[C_{A l g 1}^{(3)} \mid \Omega_{n}\right]=O\left(\mathbb{E}\left[\left|K_{n}\right| \mid \Omega_{n}\right] n \log n\right) .
$$

Next, let $C_{A l g 1}^{(4)}$ denote the complexity of computing $\hat{\tilde{\pi}}_{v}(k) \forall k \in K_{n}, v \in V_{n} \backslash K_{n}$. This has complexity $O\left(n\left|K_{n}\right|\right)$, i.e.

$$
\mathbb{E}\left[C_{A l g 1}^{(4)} \mid \Omega_{n}\right]=O\left(\mathbb{E}\left[\mid K_{n} \| \Omega_{n}\right] n\right) .
$$

Next, let $C_{A l g 1}^{(5)}$ denote the complexity of running Approx-Page- Rank $\left(v, \epsilon_{1}\right)$ for any $v \in V_{n} \backslash K_{n}$ satisfying $\hat{\tilde{\pi}}_{v}\left(K_{n}\right)<g_{n}(\epsilon / 4)$. We first observe that, by $(110), \hat{\tilde{\pi}}_{v}\left(K_{n}\right)<g_{n}(\epsilon / 4)$ implies

$$
\tilde{\pi}_{v}\left(K_{n}\right)<g_{n}(\epsilon / 4)+\frac{\left|K_{n}\right| \epsilon_{2}}{\alpha_{n}^{2}}=\frac{3}{2} g_{n}(\epsilon / 4)
$$

where we have also used $\epsilon_{2}$ as given in the statement of the theorem. Next, as in the argument leading to (94), we have

$$
\frac{3}{2} g_{n}(\epsilon / 4)<\frac{3}{2} \frac{\alpha_{n}}{\epsilon / 4}=\frac{6 \alpha_{n}}{\epsilon}, \quad g_{n}(\epsilon / 14)>\frac{\alpha_{n}(1-(\epsilon / 7))}{\epsilon / 7}=\frac{\alpha_{n}(7-\epsilon)}{\epsilon} .
$$

Hence, by assumption $\epsilon<1$, we have $\frac{3}{2} g_{n}(\epsilon / 4)<g_{n}(\epsilon / 14)$. In other words, we have shown $\hat{\tilde{\pi}}_{v}\left(K_{n}\right)<g_{n}(\epsilon / 4) \Rightarrow \tilde{\pi}_{v}\left(K_{n}\right)<g_{n}(\epsilon / 14)$. Therefore, by the argument leading to (106),

$$
\hat{\tilde{\pi}}_{v}\left(K_{n}\right)<g_{n}(\epsilon / 4) \Rightarrow\left\|\pi_{v}-\left(\alpha_{n} e_{v}^{\top}+\frac{\sum_{k \in K_{n}} \tilde{\pi}_{v}(k) \pi_{k}}{\alpha_{n}+\left(1-\alpha_{n}\right) \tilde{\pi}_{v}\left(K_{n}\right)}\right)\right\|_{1}>\frac{\epsilon}{14} .
$$

Note that the right side of the implication in (96) is the event $B_{v}\left(K_{n}, \epsilon / 14\right)$ defined in (3). Hence, the number of $v \in V_{n} \backslash K_{n}$ for which Approx-PageRank $\left(v, \epsilon_{1}\right)$ is run in Algorithm 1 satisfies

$$
\mathbb{E}\left[\mid\left\{v \in V_{n} \backslash K_{n}: 1\left(\hat{\tilde{\pi}}_{v}\left(K_{n}\right)<g_{n}(\epsilon / 4)\right)\right\} \| \Omega_{n}\right] \leq \mathbb{E}\left[\mid\left\{v \in V_{n} \backslash K_{n}: B_{v}\left(K_{n}, \epsilon / 14\right) \text { holds }\right\} \| \Omega_{n}\right] .
$$

On the other hand, by the argument in the analysis of $C_{A l g 1}^{(1)}$, the complexity of running ApproxPageRank $\left(v, \epsilon_{1}\right)$ is $O(n \log n)$ when $\Omega_{n}$ holds. Combining arguments, we obtain

$$
\mathbb{E}\left[C_{A l g 1}^{(5)} \mid \Omega_{n}\right]=O\left(\mathbb{E}\left[\mid\left\{v \in V_{n} \backslash K_{n}: B_{v}\left(K_{n}, \epsilon / 14\right) \text { holds }\right\}|| \Omega_{n}\right] n \log n\right) .
$$

Finally, let $C_{A l g 1}^{(6)}$ denote the complexity of computing $\hat{\pi}_{v}$ for all $v \in V_{n} \backslash K_{n}$ satisfying $\hat{\tilde{\pi}}_{v}\left(K_{n}\right) \geq$ $g_{n}(\epsilon / 4)$. This requires multiplying two matrices: the first has dimension $O(n) \times\left|K_{n}\right|$ and contains

$$
\left\{\hat{\tilde{\pi}}_{v}(k): v \in V_{n} \backslash K_{n} \text { s.t. } \hat{\tilde{\pi}}_{v}\left(K_{n}\right) \geq g_{n}(\epsilon / 4), k \in K_{n}\right\},
$$

and the second has dimension $\left|K_{n}\right| \times n$ and contains rows $\left\{\hat{\pi}_{k}^{l}\right\}_{k \in K_{n}}$. Note we may bound the complexity of this matrix multiplication as $n$ times the number of nonzero elements of the latter matrix. Towards this end, recall that $\hat{\pi}_{k}^{l}(u)$ is nonzero only if $u \in V_{n, k}(l)$ and $\hat{\pi}_{k}(u)$ is nonzero, so the number of nonzero elements of $\hat{\pi}_{k}^{l}$ is bounded by $\left|V_{n, k}(l)\right|$, which we can further bound as

$$
\begin{aligned}
\mathbb{E}\left[\left|V_{n, k}(l)\right| \mid \Omega_{n}\right] & \leq \mathbb{E}\left[\left|V_{n, k}(l)\right| \mid \max _{k \in K_{n}} D_{k} \leq D_{\max }, \Omega_{n}\right]+n \mathbb{P}\left[\max _{k \in K_{n}} D_{k}>D_{\max } \mid \Omega_{n}\right] \\
& =\mathbb{E}\left[\left|V_{n, k}(l)\right| \mid \max _{k \in K_{n}} D_{k} \leq D_{\max }, \Omega_{n}\right]+O\left(n^{1-\delta^{\prime}}\right),
\end{aligned}
$$

where the equality holds by the assumption in the statement of the theorem. To further bound the remaining expectation term, we use an argument from Appendix D.4 (we describe this briefly 
and refer the reader to Appendix D.4 for further details). The argument proceeds as follows. After $k \in K_{n}$ is first encountered during the graph construction and $V_{n, k}(l)$ is being constructed, we can simultaneously construct a tree of $l$ generations, adding a new node to this tree each time an instub is sampled for pairing with an outstub belonging to some $u \in V_{n, k}(l)$. By construction, $\left|V_{n, k}(l)\right|$ will be upper bounded by the number of nodes in this tree. Furthermore, the number of nodes in this tree will have the same distribution as a tree constructed via Algorithm 6 in Appendix A, which, with slight modification of (70) in Appendix A, satisfies $D_{\max } \sum_{j=1}^{l} \zeta_{n}^{j}$ with the degree sequence held fixed (here $\zeta_{n}$ is defined at the start of Appendix D.4). To summarize, we have argued $\mathbb{E}_{n}\left[\left|V_{n, k}(l)\right|\right] \leq D_{\max } \sum_{j=1}^{l} \zeta_{n}^{j}$, which further implies

$$
\begin{aligned}
& \mathbb{E}\left[\left|V_{n, k}(l)\right| \max _{k \in K_{n}} D_{k} \leq D_{\max }, \Omega_{n}\right] \\
& =\frac{1}{\mathbb{P}\left[\max _{k \in K_{n}} D_{k} \leq D_{\max }, \Omega_{n}\right]} \mathbb{E}\left[1\left(\max _{k \in K_{n}} D_{k} \leq D_{\max }, \Omega_{n}\right) \mathbb{E}_{n}\left[\left|V_{n, k}(l)\right|\right]\right] \\
& \leq \frac{1}{\mathbb{P}\left[\max _{k \in K_{n}} D_{k} \leq D_{\max }, \Omega_{n}\right]} \mathbb{E}\left[1\left(\max _{k \in K_{n}} D_{k} \leq D_{\max }, \Omega_{n}\right) D_{\max } \sum_{j=1}^{l} \zeta_{n}^{j}\right] \\
& =D_{\max } \sum_{j=1}^{l} \mathbb{E}\left[\left.\zeta_{n}^{j}\right|_{k \in K_{n}} D_{k} \leq D_{\max }, \Omega_{n}\right]=O\left(\zeta^{l}\right)=O\left(n^{1 / \rho}\right),
\end{aligned}
$$

where the penultimate equality uses $D_{\max }=O(1)$, an argument similar to (74) in Appendix D.4, and $\zeta>1$, and where the final equality uses (91) from Appendix E. Hence, by (98) and (99),

$$
\mathbb{E}\left[\mid V_{n, k}(l) \| \Omega_{n}\right]=O\left(n^{1 / \rho}+n^{1-\delta^{\prime}}\right)=O\left(n^{\max \left\{1 / \rho, 1-\delta^{\prime}\right\}}\right) .
$$

Recalling that $\left|V_{n, k}(l)\right|$ bounds the number of nonzeros of $\hat{\pi}_{k}^{l}$, that $C_{A l g 1}^{(6)}$ is bounded by $n$ times the number of nonzeros of $\left\{\hat{\pi}_{k}^{l}\right\}_{k \in K_{n}}$, we obtain

$$
\mathbb{E}\left[C_{A l g 1}^{(6)} \mid \Omega_{n}\right]=O\left(\mathbb{E}\left[\left|K_{n}\right| \mid \Omega_{n}\right] n^{1+\max \left\{1 / \rho, 1-\delta^{\prime}\right\}}\right)
$$

Finally, since $\mathbb{E}\left[\left|K_{n}\right|\right]=O\left(n^{\kappa}\right)$ by Assumption 2 and $\mathbb{P}\left[\Omega_{n}^{C}\right]=O\left(n^{-\delta}\right)$ by Assumption 1 ,

$$
\mathbb{E}\left[\mid K_{n} \| \Omega_{n}\right]=\frac{\mathbb{E}\left[K_{n} 1\left(\Omega_{n}\right)\right]}{\mathbb{P}\left[\Omega_{n}\right]} \leq \frac{\mathbb{E}\left[K_{n}\right]}{\mathbb{P}\left[\Omega_{n}\right]}=O\left(n^{\kappa}\right),
$$

and so we ultimately obtain

$$
\mathbb{E}\left[C_{A l g 1}^{(6)} \mid \Omega_{n}\right]=O\left(n^{1+\kappa+\max \left\{1 / \rho, 1-\delta^{\prime}\right\}}\right) .
$$

Now because $C_{A l g 1}=\sum_{i=1}^{6} C_{A l g 1}^{(i)}$, we have

$$
\mathbb{E}\left[C_{A l g 1} \mid \Omega_{n}\right]=\max \left\{\sum_{i=1}^{5} \mathbb{E}\left[C_{A l g 1}^{(i)} \mid \Omega_{n}\right], \mathbb{E}\left[C_{A l g 1}^{(6)} \mid \Omega_{n}\right]\right\}
$$

Using the bounds derived above for $\left\{\mathbb{E}\left[C_{A \lg 1}^{(i)} \mid \Omega_{n}\right]\right\}_{i=1}^{5}$, we have

$$
\begin{aligned}
\sum_{i=1}^{5} \mathbb{E}\left[C_{A l g 1}^{(i)} \mid \Omega_{n}\right] & =O\left(\left(\mathbb{E}\left[\mid\left\{v \in V_{n} \backslash K_{n}: B_{v}\left(K_{n}, \epsilon / 14\right) \text { holds }\right\}|| \Omega_{n}\right]+\mathbb{E}\left[\left|K_{n}\right| \mid \Omega_{n}\right]\right) n(\log n)^{3}\right) \\
& =O\left(\mathbb{E}\left[\Delta\left(K_{n}, \epsilon / 14\right) \mid \Omega_{n}\right] n(\log n)^{3}\right)=O\left(\mathbb{E}\left[\Delta\left(K_{n}, \epsilon / 14\right)\right] n(\log n)^{3}\right),
\end{aligned}
$$


where the final line holds similar to (100). Combining (101), (102), and (103) completes the proof.

We now turn to the accuracy guarantee. For this, first note that $\hat{\pi}_{v}$ is computed via Approx$\operatorname{PageRank}\left(v, \epsilon_{1}\right)$ whenever $v \in K_{n}$ or $v \in V_{n} \backslash K_{n}, \hat{\tilde{\pi}}_{v}\left(K_{n}\right)<g_{n}\left(\epsilon_{1}\right)$. In both cases, Lemma 12 ensures $\left\|\hat{\pi}_{v}-\pi_{v}\right\|_{1} \leq \epsilon_{1}=\epsilon / 4<\epsilon$. Thus, it only remains to show $\left\|\hat{\pi}_{v}-\pi_{v}\right\|_{1} \leq \epsilon$ when $v \in V_{n} \backslash K_{n}$ and $\hat{\tilde{\pi}}_{v}\left(K_{n}\right) \geq g_{n}\left(\epsilon_{1}\right)$, in which case we instead compute $\hat{\pi}_{v}$ as

$$
\hat{\pi}_{v}=\alpha_{n} e_{v}^{\top}+\frac{\sum_{k \in K_{n}} \hat{\tilde{\pi}}_{v}(k) \hat{\pi}_{k}^{l}}{\alpha_{n}+\left(1-\alpha_{n}\right) \hat{\tilde{\pi}}_{v}\left(K_{n}\right)} .
$$

We first note that by (56) and the definition of $\hat{\tilde{\pi}}_{v}$, we have

$$
\tilde{\pi}_{v}\left(K_{n}\right)=\frac{\alpha_{n} \mu_{v}\left(K_{n}\right)}{1-\left(1-\alpha_{n}\right) \mu_{v}\left(K_{n}\right)} \geq \frac{\alpha_{n} \hat{\mu}_{v}\left(K_{n}\right)}{1-\left(1-\alpha_{n}\right) \hat{\mu}_{v}\left(K_{n}\right)}=\hat{\tilde{\pi}}_{v}\left(K_{n}\right)
$$

where the inequality holds because the left side is increasing in $\mu_{v}\left(K_{n}\right)$ and since $\mu_{v}\left(K_{n}\right) \geq \hat{\mu}_{v}\left(K_{n}\right)$ by Lemma 13. Thus, $\hat{\tilde{\pi}}_{v}\left(K_{n}\right) \geq g_{n}\left(\epsilon_{1}\right)$ implies $\tilde{\pi}_{v}\left(K_{n}\right) \geq g_{n}\left(\epsilon_{1}\right)$ as well; furthermore, some simple algebra, along with (52) in Appendix D.2, shows

$$
\tilde{\pi}_{v}\left(K_{n}\right) \geq g_{n}\left(\epsilon_{1}\right) \Leftrightarrow\left\|\pi_{v}-\left(\alpha_{n} e_{v}^{\top}+\frac{\sum_{k \in K_{n}} \tilde{\pi}_{v}(k) \pi_{k}}{\alpha_{n}+\left(1-\alpha_{n}\right) \tilde{\pi}_{v}\left(K_{n}\right)}\right)\right\|_{1} \leq \epsilon_{1}=\frac{\epsilon}{4},
$$

where the equality holds by the statement of the theorem. Then

$$
\begin{aligned}
\left\|\pi_{v}-\hat{\pi}_{v}\right\|_{1} \leq & \left\|\pi_{v}-\left(\alpha_{n} e_{v}^{\top}+\frac{\sum_{k \in K_{n}} \tilde{\pi}_{v}(k) \pi_{k}}{\alpha_{n}+\left(1-\alpha_{n}\right) \tilde{\pi}_{v}\left(K_{n}\right)}\right)\right\|_{1}+\left\|\frac{\sum_{k \in K_{n}} \tilde{\pi}_{v}(k)}{\alpha_{n}+\left(1-\alpha_{n}\right) \tilde{\pi}_{v}\left(K_{n}\right)}\left(\pi_{k}-\hat{\pi}_{k}^{l}\right)\right\|_{1} \\
& +\left\|\frac{\sum_{k \in K_{n}} \hat{\pi}_{k}^{l}}{\alpha_{n}+\left(1-\alpha_{n}\right) \tilde{\pi}_{v}\left(K_{n}\right)}\left(\tilde{\pi}_{v}(k)-\hat{\tilde{\pi}}_{v}(k)\right)\right\|_{1} \\
& +\left\|\sum_{k \in K_{n}} \hat{\tilde{\pi}}_{v}(k) \hat{\pi}_{k}^{l}\left(\frac{1}{\alpha_{n}+\left(1-\alpha_{n}\right) \tilde{\pi}_{v}\left(K_{n}\right)}-\frac{1}{\alpha_{n}+\left(1-\alpha_{n}\right) \hat{\tilde{\pi}}_{v}\left(K_{n}\right)}\right)\right\|_{1} \\
\leq & \frac{\epsilon}{4}+\max _{k \in K_{n}}\left\|\pi_{k}-\hat{\pi}_{k}^{l}\right\|_{1}+\frac{\tilde{\pi}_{v}\left(K_{n}\right)-\hat{\tilde{\pi}}_{v}\left(K_{n}\right)}{\alpha_{n}+\left(1-\alpha_{n}\right) \tilde{\pi}_{v}\left(K_{n}\right)} \\
& +\frac{\left(1-\alpha_{n}\right) \hat{\tilde{\pi}}_{v}\left(K_{n}\right)\left(\tilde{\pi}_{v}\left(K_{n}\right)-\hat{\tilde{\pi}}_{v}\left(K_{n}\right)\right)}{\left(\alpha_{n}+\left(1-\alpha_{n}\right) \tilde{\pi}_{v}\left(K_{n}\right)\right)\left(\alpha_{n}+\left(1-\alpha_{n}\right) \hat{\tilde{\pi}}_{v}\left(K_{n}\right)\right)},
\end{aligned}
$$

where the first inequality is the triangle inequality, and the second uses (106) for the first term and the triangle inequality for the other terms (in bounding the final two terms, we have also used the fact that for each $k \in K_{n},\left\|\hat{\pi}_{k}^{l}\right\|_{1} \leq 1$ and $\left.\tilde{\pi}_{v}(k) \geq \hat{\tilde{\pi}}_{v}(k)\right)$. We next derive bounds on the final three terms in (107). For the second term, we observe

$$
\max _{k \in K_{n}}\left\|\pi_{k}-\hat{\pi}_{k}^{l}\right\|_{1} \leq \max _{k \in K_{n}}\left\|\pi_{k}-\hat{\pi}_{k}\right\|_{1}+\max _{k \in K_{n}}\left\|\hat{\pi}_{k}-\hat{\pi}_{k}^{l}\right\|_{1} \leq \epsilon_{1}+\max _{k \in K_{n}} \pi_{k}\left(V_{n} \backslash V_{n, k}(l)\right) \leq \epsilon_{1}+\tau,
$$

where the first inequality is the triangle inequality, the second uses Lemma 12 and the fact that $\hat{\pi}_{k}(v)=\hat{\pi}_{k}^{l}(v)$ for $v \in V_{n, k}(l)$ and $\hat{\pi}_{k}(v) \leq \pi_{k}(v), \hat{\pi}_{k}^{l}(v)=0$ for other $v$, and the third follows by the argument leading to (90) in Appendix E. For the fourth term in (107), first note that by $\alpha_{n}>0$ and $\left.\hat{\tilde{\pi}}_{v}\left(K_{n}\right)\right) \leq 1$,

$$
\frac{\left(1-\alpha_{n}\right) \hat{\tilde{\pi}}_{v}\left(K_{n}\right)}{\left(\alpha_{n}+\left(1-\alpha_{n}\right) \hat{\tilde{\pi}}_{v}\left(K_{n}\right)\right)}<1
$$


and so the final two terms in (107) can be bounded as

$$
\begin{aligned}
& \frac{\tilde{\pi}_{v}\left(K_{n}\right)-\hat{\tilde{\pi}}_{v}\left(K_{n}\right)}{\alpha_{n}+\left(1-\alpha_{n}\right) \tilde{\pi}_{v}\left(K_{n}\right)}+\frac{\left(1-\alpha_{n}\right) \hat{\tilde{\pi}}_{v}\left(K_{n}\right)\left(\tilde{\pi}_{v}\left(K_{n}\right)-\hat{\tilde{\pi}}_{v}\left(K_{n}\right)\right)}{\left(\alpha_{n}+\left(1-\alpha_{n}\right) \tilde{\pi}_{v}\left(K_{n}\right)\right)\left(\alpha_{n}+\left(1-\alpha_{n}\right) \hat{\tilde{\pi}}_{v}\left(K_{n}\right)\right)} \\
& \quad \leq \frac{2\left(\tilde{\pi}_{v}\left(K_{n}\right)-\hat{\tilde{\pi}}_{v}\left(K_{n}\right)\right)}{\alpha_{n}+\left(1-\alpha_{n}\right) \tilde{\pi}_{v}\left(K_{n}\right)} \leq \frac{2\left(\tilde{\pi}_{v}\left(K_{n}\right)-\hat{\tilde{\pi}}_{v}\left(K_{n}\right)\right)}{g_{n}(\epsilon / 4)},
\end{aligned}
$$

where for the second inequality, we have used $\alpha_{n}+\left(1-\alpha_{n}\right) \tilde{\pi}_{v}\left(K_{n}\right) \geq \tilde{\pi}_{v}\left(K_{n}\right) \geq g_{n}(\epsilon / 4)$ by assumption. Furthermore, we note

$$
\begin{aligned}
\tilde{\pi}_{v}\left(K_{n}\right)-\hat{\tilde{\pi}}_{v}\left(K_{n}\right) & =\frac{\alpha_{n} \mu_{v}\left(K_{n}\right)}{1-\left(1-\alpha_{n}\right) \mu_{v}\left(K_{n}\right)}-\frac{\alpha_{n} \hat{\mu}_{v}\left(K_{n}\right)}{1-\left(1-\alpha_{n}\right) \hat{\mu}_{v}\left(K_{n}\right)} \\
& =\frac{\alpha_{n}\left(\mu_{v}\left(K_{n}\right)-\hat{\mu}_{v}\left(K_{n}\right)\right)}{\left(1-\left(1-\alpha_{n}\right) \mu_{v}\left(K_{n}\right)\right)\left(1-\left(1-\alpha_{n}\right) \hat{\mu}_{v}\left(K_{n}\right)\right)} \leq \frac{\left|K_{n}\right| \epsilon_{2}}{\alpha_{n}^{2}},
\end{aligned}
$$

where the first equality holds by (105), and the inequality uses Lemma 13 and the fact that, by (56),

$$
\mu_{v}\left(K_{n}\right)=\frac{\tilde{\pi}_{v}\left(K_{n}\right)}{\alpha_{n}+\left(1-\alpha_{n}\right) \tilde{\pi}_{v}\left(K_{n}\right)} \leq 1,
$$

and a similar argument implies $\hat{\mu}_{v}\left(K_{n}\right) \leq 1$. Hence, combining (107), (108), (109), and (110), we have shown that when $v \in V_{n} \backslash K_{n}$ and $\hat{\tilde{\pi}}_{v}\left(K_{n}\right) \geq g_{n}\left(\epsilon_{1}\right)$, i.e. when $\hat{\pi}_{v}$ is computed via (104),

$$
\left\|\pi_{v}-\hat{\pi}_{v}\right\|_{1} \leq \frac{\epsilon}{4}+\epsilon_{1}+\tau+\frac{2\left|K_{n}\right| \epsilon_{2}}{\alpha_{n}^{2} g_{n}(\epsilon / 4)} \leq \epsilon
$$

where the final inequality holds by our assumptions on $\epsilon_{1}, \epsilon_{2}, \tau$. This completes the proof.

\section{G.3 Precomputation variant of Algorithm 1}

We begin by analyzing the accuracy of the precomputation variant. For this, we first note that the estimate of $\pi_{v}, v \notin K_{n}$ is computed in the same manner as in Algorithm 1, so the accuracy guarantee of Theorem 2 holds for such $v$. However, for $k \in K_{n}$, the precomputation variant instead returns $\hat{\pi}_{k}^{l}$, so the accuracy guarantee does not apply. Nevertheless, by (108), we have

$$
\left\|\pi_{k}-\hat{\pi}_{k}^{l}\right\|_{1} \leq \epsilon_{1}+\tau \leq \epsilon / 2
$$

where the second inequality holds by the assumptions in Theorem 2 . Hence, all estimates returned by the variant satisfy the accuracy guarantee claimed in the main text.

We next consider the space complexity for storing $\left\{\hat{\pi}_{k}^{l}\right\}_{k \in K_{n}}$ and $\left\{\hat{\mu}_{k}(k)\right\}_{u \in V_{n}, k \in K_{n}}$ from the offline stage. Trivially, $n n z\left(\hat{\pi}_{k}^{l}\right) \leq n$ and $n n z\left(\left\{\hat{\mu}_{k}(k)\right\}_{u \in V_{n}}\right) \leq n \forall k \in K_{n}$, where $n n z(x)$ denote the number of nonzero elements of the vector $x$. Hence, the overall storage is at most $2 n\left|K_{n}\right|$, which is $O\left(n^{1+\kappa}\right)$ in expectation.

Finally, we consider the complexity of running the online stage for $v^{*} \sim V_{n}$ uniformly. If $v^{*} \in K_{n}$, no computation is required, so this complexity is negligible. If $v^{*} \in V_{n} \backslash K_{n}$, this complexity is simply $1 /\left|V_{n} \backslash K_{n}\right|$ times the complexity of running Lines 7-12 $\forall v \in V_{n} \backslash K_{n}$ in Algorithm 1. By the analysis in Appendix G.2 (specifically, by (95), (97), and (101)), this latter quantity is

$$
\begin{aligned}
\sum_{i=4}^{6} \mathbb{E}\left[C_{A l g 1}^{(i)} \mid \Omega_{n}\right]= & O\left(\mathbb{E}\left[\mid K_{n} \| \Omega_{n}\right] n\right)+O\left(\mathbb{E}\left[\mid\left\{v \in V_{n} \backslash K_{n}: B_{v}\left(K_{n}, \epsilon / 14\right) \text { holds }\right\} \| \Omega_{n}\right] n \log n\right) \\
& +O\left(n^{1+\kappa+\max \left\{1 / \rho, 1-\delta^{\prime}\right\}}\right) \\
= & O\left(\max \left\{\Delta\left(K_{n}, \epsilon / 14\right) n \log n, n^{1+\kappa+\max \left\{1 / \rho, 1-\delta^{\prime}\right\}}\right\}\right) .
\end{aligned}
$$


Hence, with $\left|V_{n} \backslash K_{n}\right|=O(n)$ in expectation, the complexity of running the online stage for $v^{*} \sim V_{n}$ uniformly is

$$
O\left(\max \left\{\Delta\left(K_{n}, \epsilon / 14\right) \log n, n^{\kappa+\max \left\{1 / \rho, 1-\delta^{\prime}\right\}}\right\}\right)
$$

\section{H PROOF OF PROPOSITION 3}

Assume $n \geq v$, so that $\pi_{v}, P_{v}$ are given by Definition 1 . For such $n$, we claim that for any realization of $G_{n}$ and any $i \in \mathbb{N}$,

$$
P_{v}^{i}=\alpha_{n} 1_{n} e_{v}^{T} \sum_{j=0}^{i-1}\left(1-\alpha_{n}\right)^{j} P^{j}+\left(1-\alpha_{n}\right)^{i} P^{i}
$$

We prove (111) inductively: it holds by definition for $i=1$, and if it holds for some general $i$,

$$
\begin{aligned}
P_{v}^{i+1} & =P_{v}\left(\alpha_{n} 1_{n} e_{v}^{T} \sum_{j=0}^{i-1}\left(1-\alpha_{n}\right)^{j} P^{j}+\left(1-\alpha_{n}\right)^{i} P^{i}\right) \\
& =\alpha_{n}\left(P_{v} 1_{n}\right) e_{v}^{\top} \sum_{j=0}^{i-1}\left(1-\alpha_{n}\right)^{j} P^{j}+\left(\alpha_{n} 1_{n} e_{v}^{\top}+\left(1-\alpha_{n}\right) P\right)\left(1-\alpha_{n}\right)^{i} P^{i} \\
& =\alpha_{n} 1_{n} e_{v}^{T} \sum_{j=0}^{i-1}\left(1-\alpha_{n}\right)^{j} P^{j}+\alpha_{n} 1_{n} e_{v}^{\top}\left(1-\alpha_{n}\right)^{i} P^{i}+\left(1-\alpha_{n}\right)^{i+1} P^{i+1} \\
& =\alpha_{n} 1_{n} e_{v}^{T} \sum_{j=0}^{i}\left(1-\alpha_{n}\right)^{j} P^{j}+\left(1-\alpha_{n}\right)^{i+1} P^{i+1},
\end{aligned}
$$

where the first equality holds by the inductive hypothesis, the second uses the definition of $P_{v}$, and the third uses row stochasticity of $P_{v}$; the other manipulations are straightforward. We next write

$$
\begin{aligned}
\pi_{v} & =\pi_{v} P_{v}^{m}=\alpha_{n} e_{v}^{T} \sum_{j=0}^{m-1}\left(1-\alpha_{n}\right)^{j} P^{j}+\left(1-\alpha_{n}\right)^{m} \pi_{v} P^{m} \\
& =\pi_{v} P^{m}+\alpha_{n} e_{v}^{T} \sum_{j=0}^{m-1}\left(1-\alpha_{n}\right)^{j} P^{j}+\left(\left(1-\alpha_{n}\right)^{m}-1\right) \pi_{v} P^{m},
\end{aligned}
$$

where the equalities follow by global balance $\left(\pi_{v}=\pi_{v} P_{v}\right),(111)$ and the fact $\pi_{v}$ sums to 1 , and adding/subtracting $\pi_{v} P^{m}$, respectively. Next, we have for any $w \in V_{n}$,

$$
\begin{aligned}
\left\|\pi-\pi_{v}\right\|_{1} & \leq\left\|\pi-e_{w} P^{m}\right\|_{1}+\left\|e_{w} P^{m}-\pi_{v} P^{m}\right\|_{1}+\left\|\pi_{v} P^{m}-\pi_{v}\right\|_{1} \\
& =\left\|\pi-e_{w} P^{m}\right\|_{1}+\left\|e_{w} P^{m}-\pi_{v} P^{m}\right\|_{1}+2\left(1-\left(1-\alpha_{n}\right)^{m}\right),
\end{aligned}
$$

where the inequality is the triangle inequality, and the equality follows by (112) and the fact that $P$ is row stochastic. Again using the triangle inequality, as well as the fact that $\pi_{v}$ sums to 1 and 
convexity of $\|\cdot\|_{1}$, we can write

$$
\begin{aligned}
\left\|e_{w} P^{m}-\pi_{v} P^{m}\right\|_{1} & \leq\left\|e_{w} P^{m}-\pi\right\|_{1}+\left\|\sum_{w^{\prime} \in V_{n}} \pi_{v}\left(w^{\prime}\right)\left(e_{w^{\prime}}^{\top} P^{m}-\pi\right)\right\|_{1} \\
& \leq\left\|e_{w} P^{m}-\pi\right\|_{1}+\sum_{w^{\prime} \in V_{n}} \pi_{v}\left(w^{\prime}\right)\left\|e_{w^{\prime}}^{\top} P^{m}-\pi\right\|_{1} \\
& \leq\left\|e_{w} P^{m}-\pi\right\|_{1}+\left(\sum_{w^{\prime} \in V_{n}} \pi_{v}\left(w^{\prime}\right)\right)\left(\max _{w^{\prime} \in V_{n}}\left\|e_{w^{\prime}}^{\top} P^{m}-\pi\right\|_{1}\right) \\
& =\left\|e_{w} P^{m}-\pi\right\|_{1}+\max _{w^{\prime} \in V_{n}}\left\|e_{w^{\prime}}^{\top} P^{m}-\pi\right\|_{1} \leq 2 \max _{w^{\prime} \in V_{n}}\left\|e_{w^{\prime}}^{\top} P^{m}-\pi\right\|_{1} .
\end{aligned}
$$

We may then combine (113) and (114) to obtain

$$
\left\|\pi_{v}-\pi\right\|_{1} \leq 3 \max _{w \in V_{n}}\left\|e_{w} P^{m}-\pi\right\|_{1}+2\left(1-\left(1-\alpha_{n}\right)^{m}\right) .
$$

Finally, by Lemma 14 below, $\exists N \in \mathbb{N}$ s.t. $2\left(1-\left(1-\alpha_{n}\right)^{m}\right)<\epsilon \forall n \geq N$. Hence, taking $n \geq \max \{v, N\}$, we have shown that for any realization of $G_{n}$,

$$
\left\|\pi_{v}-\pi\right\|_{1} \leq 3 \max _{w \in V_{n}}\left\|e_{w}^{\top} P^{m}-\pi\right\|_{1}+\epsilon
$$

which completes the proof.

LEMMA 14. If $m=\Theta(\log n)$ and $\alpha_{n}=o\left(\frac{1}{\log n}\right)$, then

$$
\lim _{n \rightarrow \infty}\left(1-\alpha_{n}\right)^{m}=1 \text {. }
$$

Proof. We will show $\lim _{n \rightarrow \infty}\left(1-\alpha_{n}\right)^{k \log n}=1$ for any $k \in(0, \infty)$, from which the lemma follows. For this, first let $\epsilon, \delta>0$. Suppose $\liminf _{n \rightarrow \infty}\left(1-\alpha_{n}\right)^{k \log n}=c$ for some $c \in[0,1)$. Then $\exists N_{1} \in \mathbb{N}$ s.t.

$$
c+\epsilon>\inf _{n^{\prime} \geq n}\left(1-\alpha_{n^{\prime}}\right)^{k \log n^{\prime}} \forall n \geq N_{1} .
$$

On the other hand, since $\lim _{n \rightarrow \infty} \alpha_{n} \log n=0, \exists N_{2}$ s.t.

$$
\begin{aligned}
& \alpha_{n} \log n<\delta \forall n \geq N_{2} \\
& \Rightarrow\left(1-\alpha_{n}\right)^{k \log n}>\left(1-\frac{\delta}{\log n}\right)^{k \log n} \forall n \geq N_{2} \\
& \Rightarrow \inf _{n^{\prime} \geq n}\left(1-\alpha_{n^{\prime}}\right)^{k \log n^{\prime}} \geq \inf _{n^{\prime} \geq n}\left(1-\frac{\delta}{\log n^{\prime}}\right)^{k \log n^{\prime}} \forall n \geq N_{2} .
\end{aligned}
$$

(Technically, we also need $n$ large enough that $\delta<\log n$ in the above derivation.) Finally, since $\lim _{n \rightarrow \infty}\left(1-\frac{\delta}{\log n}\right)^{k \log n}=e^{-\delta k}, \exists N_{3}$ s.t.

$$
\left(1-\frac{\delta}{\log n}\right)^{k \log n}>e^{-\delta k}-\epsilon \forall n \geq N_{3} \Rightarrow \inf _{n^{\prime} \geq n}\left(1-\frac{\delta}{\log n^{\prime}}\right)^{k \log n^{\prime}} \geq e^{-\delta k}-\epsilon \forall n \geq N_{3} .
$$

Combining the above, we obtain $\forall n \geq \max \left\{N_{1}, N_{2}, N_{3}\right\}$,

$$
c+\epsilon>\inf _{n^{\prime} \geq n}\left(1-\alpha_{n^{\prime}}\right)^{k \log n^{\prime}} \geq \inf _{n^{\prime} \geq n}\left(1-\frac{\delta}{\log n^{\prime}}\right)^{k \log n^{\prime}} \geq e^{-\delta k}-\epsilon .
$$

Since this argument holds $\forall \epsilon, \delta>0$, let $\epsilon \in\left(0, \frac{1-c}{2}\right)$, and set $\delta=-\frac{\log (c+2 \epsilon)}{k}$. (Note $\epsilon, \delta \in(0, \infty)$ by $c \in[0,1), k \in(0, \infty)$.) Then (115) implies $c+\epsilon<c+\epsilon$, a contradiction. We have therefore 
shown $\liminf _{n \rightarrow \infty}\left(1-\alpha_{n}\right)^{k \log n} \notin[0,1)$; since clearly this term is nonnegative, we conclude $\liminf _{n \rightarrow \infty}\left(1-\alpha_{n}\right)^{k \log n} \geq 1$. Finally, noting the lim sup is upper bounded by 1 completes the proof.

\section{PROOF OF PROPOSITION 2}

We begin with some auxiliary lemmas in Appendix I.1. Next, we bound the probabilities for each of the events defined in Assumption 1 in Appendix I.2. Finally, we use these bounds to prove Proposition 2 in Appendix I.3. We also note that several proofs in Appendix I.2 rely crucially on the observation

$$
D_{v} \mid\left\{N_{w}\right\}_{w \in V_{n}}=1+\operatorname{Binomial}\left(\sum_{w \in V_{n}}\left(N_{w}-1\right), \frac{1}{n}\right),
$$

and similarly, whenever $v \neq v^{\prime}$,

$$
D_{v} \mid\left\{N_{w}\right\}_{w \in V_{n}}, D_{v^{\prime}}=1+\operatorname{Binomial}\left(\sum_{w \in V_{n}}\left(N_{w}-1\right)-D_{v^{\prime}}, \frac{1}{n-1}\right) .
$$

\section{I.1 Some simple lemmas}

Throughout the proof, for $d>1$, we let $z(d)=\sum_{i=1}^{\infty} i^{-d} \in(0, \infty)$ (note this is the Riemann zeta function, but we use $z$ to avoid confusion with $\zeta$ defined in Assumption 1). We begin with three lemmas regarding this function, which will be used throughout.

LEMmA 15. For every $b, b^{\prime} \in \mathbb{N}$ s.t. $b<b^{\prime}$, and for every $d_{1}>d_{2}$,

$$
\frac{\sum_{i=1}^{b} i^{-d_{2}}}{\sum_{i=1}^{b} i^{-d_{1}}}<\frac{\sum_{i=1}^{b^{\prime}} i^{-d_{2}}}{\sum_{i=1}^{b^{\prime}} i^{-d_{1}}}
$$

If in addition $d_{2}>1$,

$$
\frac{\sum_{i=1}^{b} i^{-d_{2}}}{\sum_{i=1}^{b} i^{-d_{1}}}<\frac{z\left(d_{2}\right)}{z\left(d_{1}\right)}
$$

Proof. We observe

$$
\begin{aligned}
& \frac{\sum_{i=1}^{b} i^{-d_{2}}}{\sum_{i=1}^{b} i^{-d_{1}}}<\frac{\sum_{i=1}^{b} i^{-d_{1}}(b+1)^{d_{1}-d_{2}}}{\sum_{i=1}^{b} i^{-d_{1}}}=(b+1)^{d_{1}-d_{2}} \\
& \Rightarrow \sum_{i=1}^{b} i^{-d_{2}}<\sum_{i=1}^{b} i^{-d_{1}}(b+1)^{d_{1}-d_{2}} \\
& \Rightarrow \sum_{i=1}^{b} i^{-d_{2}}(b+1)^{-d_{1}}<\sum_{i=1}^{b} i^{-d_{1}}(b+1)^{-d_{2}} \\
& \Rightarrow \sum_{i=1}^{b} i^{-d_{2}}\left(\sum_{i=1}^{b} i^{-d_{1}}+(b+1)^{-d_{1}}\right)<\sum_{i=1}^{b} i^{-d_{1}}\left(\sum_{i=1}^{b} i^{-d_{2}}+(b+1)^{-d_{2}}\right) \\
& \Rightarrow \frac{\sum_{i=1}^{b} i^{-d_{2}}}{\sum_{i=1}^{b} i^{-d_{1}}}<\frac{\sum_{i=1}^{b} i^{-d_{2}}+(b+1)^{-d_{2}}}{\sum_{i=1}^{b} i^{-d_{1}}+(b+1)^{-d_{1}}}=\frac{\sum_{i=1}^{b+1} i^{-d_{2}}}{\sum_{i=1}^{b+1} i^{-d_{1}}} .
\end{aligned}
$$

Applying this inequality $b^{\prime}-b-1$ more times yields the first part of the lemma. The second part of the lemma follows because, when $d_{1}, d_{2}>1, \sum_{i=1}^{b} i^{-d_{2}} / \sum_{i=1}^{b} i^{-d_{1}} \rightarrow z\left(d_{2}\right) / z\left(d_{1}\right)$ as $b \rightarrow \infty$. 
Lemma 16. For every $b \in \mathbb{N}$ and everyd $>1$,

$$
b^{1-d}(z(d)-1)<\sum_{i=b+1}^{\infty} i^{-d}<b^{1-d} z(d) .
$$

Proof. For the upper bound, we have

$$
\begin{aligned}
\sum_{i=b+1}^{\infty} i^{-d} & =\left((b+1)^{-d}+\cdots+(2 b)^{-d}\right)+\left((2 b+1)^{-d}+\cdots+(3 b)^{-d}\right)+\cdots \\
& <b(b)^{-d}+b(2 b)^{-d}+\cdots=b^{1-d}\left(1+2^{-d}+\cdots\right)=b^{1-d} z(d) .
\end{aligned}
$$

Similarly, for the lower bound,

$$
\sum_{i=b+1}^{\infty} i^{-d}>b(2 b)^{-d}+b(3 b)^{-d}+\cdots=b^{1-d}\left(2^{-d}+3^{-d}+\cdots\right)=b^{1-d}(z(d)-1) .
$$

Lemma 17. For every $b \in \mathbb{N}$, the following hold:

$$
\begin{aligned}
& d \in(0,1) \Rightarrow \sum_{i=1}^{b} i^{-d} \in\left(\frac{(b+1)^{1-d}-1}{1-d}, \frac{b^{1-d}}{1-d}\right) \\
& d \in(-\infty, 0) \Rightarrow \sum_{i=1}^{b} i^{-d} \in\left(\frac{b^{1-d}}{1-d}, \frac{(b+1)^{1-d}-1}{1-d}\right)
\end{aligned}
$$

Clearly, for $d=0, \sum_{i=1}^{b} i^{-d}=b=b^{1-d}$. Hence, ifd is constant as $b \rightarrow \infty, \sum_{i=1}^{b} i^{-d}=\Theta\left(b^{1-d}\right) \forall d<1$.

Proof. For $d \in(0,1)$, we have

$$
\begin{aligned}
& \sum_{i=1}^{b} i^{-d}=\sum_{i=1}^{b} i^{-d} \int_{x=i-1}^{i} d x<\sum_{i=1}^{b} \int_{x=i-1}^{i} x^{-d} d x=\frac{b^{1-d}}{1-d} \\
& \sum_{i=1}^{b} i^{-d}=\sum_{i=1}^{b} i^{-d} \int_{x=i}^{i+1} d x>\sum_{i=1}^{b} \int_{x=i}^{i+1} x^{-d} d x=\frac{(b+1)^{1-d}-1}{1-d} .
\end{aligned}
$$

For $d \in(-\infty, 0)$, simply reverse the inequalities.

We also establish the following lemma.

LEMma 18. We have

$$
\mathbb{P}\left[\sum_{v \in V_{n}}\left(1-U_{v}\right) \notin\left(n^{\kappa}-n^{2 \kappa / 3}, n^{\kappa}+n^{2 \kappa / 3}\right)\right] \leq 2 \exp \left(-\frac{n^{\kappa / 3}}{3}\right) .
$$

Proof. We will use the following version of the Chernoff bound (see Theorem 1.1 in [24]): for $Z=\sum_{i} Z_{i}$, where $\left\{Z_{i}\right\}$ are independently distributed in $[0,1]$, and for every $\epsilon \in(0,1)$,

$$
\mathbb{P}[|Z-\mathbb{E}[Z]|>\epsilon \mathbb{E}[Z]] \leq 2 \exp \left(-\frac{\epsilon^{2} \mathbb{E}[Z]}{3}\right) .
$$

In our setting, $\left\{\left(1-U_{v}\right)\right\}_{v \in V_{n}}$ are independent $\operatorname{Bernoulli}\left(n^{\kappa-1}\right)$ random variables, so $\mathbb{E}\left[\sum_{v \in V_{n}}(1-\right.$ $\left.\left.U_{v}\right)\right]=n^{\kappa}$. Hence, by (116),

$$
\mathbb{P}\left[\sum_{v \in V_{n}}\left(1-U_{v}\right) \notin\left(n^{\kappa}-n^{2 \kappa / 3}, n^{\kappa}+n^{2 \kappa / 3}\right)\right] \leq 2 \exp \left(-\frac{n^{-2 \kappa / 3} n^{\kappa}}{3}\right)=2 \exp \left(-\frac{n^{\kappa / 3}}{3}\right) .
$$




\section{I.2 Probability bounds for the events $\Omega_{n, i}^{C}$}

The next six lemmas bound the probabilities of the events $\Omega_{n, i}^{C}$ for $i \in\{1,2, \ldots, 6\}$, as well as identifying the constants $\eta_{1}, \eta_{2}, \eta_{3}, \zeta^{*}, \lambda^{*}$, and $p$. In the proofs, we use $\operatorname{Var}(X)=\mathbb{E}\left[X^{2}\right]-(\mathbb{E}[X])^{2}$ to denote the variance of $X$ and $\operatorname{Cov}\left(X_{1}, X_{2}\right)=\mathbb{E}\left[X_{1} X_{2}\right]-\mathbb{E}\left[X_{1}\right] \mathbb{E}\left[X_{2}\right]$ to denote the covariance of random variables $X_{1}, X_{2}$.

LEMma 19. Let

$$
\eta_{1}=\frac{z\left(c_{1}-1\right)}{z\left(c_{1}\right)}+\frac{1}{\left(2-c_{2}\right) z\left(c_{2}\right)} .
$$

(Note $\eta_{1}$ is well-defined since $c_{1}>3, c_{2}>1$ by assumption.) Then if $\gamma \in\left(0, \min \left\{l_{1}\left(c_{1}-2\right), l_{2}\left(c_{2}-\right.\right.\right.$ $1), 1-\kappa\})$, we have

$$
\mathbb{P}\left[\left|\frac{1}{n} \sum_{v \in V_{n}} N_{v}-\eta_{1}\right|>n^{-\gamma}\right]=O\left(n^{l_{2}+2 \gamma-1}\right) .
$$

Proof. First, for $v \in V_{n}$, we have by definition

$$
\begin{aligned}
\mathbb{E}\left[N_{v}\right]-\eta_{1}= & \left.\left(1-n^{\kappa-1}\right) \frac{\sum_{i=1}^{\left\lceil n^{l_{1}}\right\rceil} i^{1-c_{1}}}{\sum_{i=1}^{\left\lceil n_{1} l_{1}\right.} i^{-c_{1}}}-\frac{z\left(c_{1}-1\right)}{z\left(c_{1}\right)}\right) \\
& +\left(n^{\kappa-1} \frac{\sum_{i=1}^{\left\lceil n_{2}\right\rceil} i^{1-c_{2}}}{\sum_{i=1}^{\left\lceil n_{2}\right\rceil} i^{-c_{2}}}-\frac{1}{\left(2-c_{2}\right) z\left(c_{2}\right)}\right) .
\end{aligned}
$$

We seek upper and lower bounds on (117) and (118). For (117), we use Lemma 15 and $1-n^{\kappa-1}<1$ to immediately obtain an upper bound of zero. For a lower bound, note

$$
\begin{aligned}
& \left(1-n^{\kappa-1}\right) \frac{\sum_{i=1}^{\left\lceil n^{l_{1}}\right\rceil} i^{1-c_{1}}}{\sum_{i=1}^{\left\lceil n^{l_{1}}\right\rceil} i^{-c_{1}}}-\frac{z\left(c_{1}-1\right)}{z\left(c_{1}\right)} \\
& >\frac{\left(1-n^{\kappa-1}\right) \sum_{i=1}^{\left\lceil n^{l_{1}}\right\rceil} i^{1-c_{1}}-z\left(c_{1}-1\right)}{z\left(c_{1}\right)}=\frac{-\left(1-n^{\kappa-1}\right) \sum_{i=\left\lceil n^{l_{1}}\right\rceil+1}^{\infty} i^{1-c_{1}}-n^{\kappa-1} z\left(c_{1}-1\right)}{z\left(c_{1}\right)} \\
& >\frac{-\left(1-n^{\kappa-1}\right)\left\lceil n^{l_{1}}\right\rceil^{2-c_{1}} z\left(c_{1}-1\right)-n^{\kappa-1} z\left(c_{1}-1\right)}{z\left(c_{1}\right)}>\frac{-z\left(c_{1}-1\right)}{z\left(c_{1}\right)}\left(\left\lceil n^{l_{1}}\right\rceil^{2-c_{1}}+n^{\kappa-1}\right),
\end{aligned}
$$

where the first inequality is simply $\sum_{i=1}^{\left\lceil n^{l_{1}}\right\rceil} i^{-c_{1}}<z\left(c_{1}\right)$, the second uses Lemma 16 , and the third is simply $\left(1-n^{\kappa-1}\right)<1$. To summarize, we have shown

$$
\left|\left(1-n^{\kappa-1}\right) \frac{\sum_{i=1}^{\left\lceil n^{l_{1}}\right\rceil} i^{1-c_{1}}}{\sum_{i=1}^{\left\lceil n_{1}\right\rceil} i^{-c_{1}}}-\frac{z\left(c_{1}-1\right)}{z\left(c_{1}\right)}\right|=O\left(n^{-\min \left\{l_{1}\left(c_{1}-2\right), 1-\kappa\right\}}\right) .
$$

For (118) we begin with the upper bound. First observe

$$
n^{\kappa-1} \frac{\sum_{i=1}^{\left\lceil n^{l_{2}}\right\rceil} i^{1-c_{2}}}{\sum_{i=1}^{\left\lceil n_{2}^{l_{2}}\right\rceil} i^{-c_{2}}}<n^{\kappa-1} \frac{\left\lceil n^{l_{2}}\right\rceil^{2-c_{2}} /\left(2-c_{2}\right)}{z\left(c_{2}\right)-\left\lceil n^{l_{2}}\right\rceil^{1-c_{2}} z\left(c_{2}\right)}=\frac{n^{\kappa-1}\left\lceil n^{l_{2}}\right\rceil^{2-c_{2}}}{\left(2-c_{2}\right) z\left(c_{2}\right)\left(1-\left\lceil n^{l_{2}}\right\rceil^{1-c_{2}}\right)},
$$


where the inequality uses Lemma 17 in the numerator and Lemma 16 in the denominator. Then

$$
\begin{aligned}
n^{\kappa-1} \frac{\sum_{i=1}^{\left\lceil n^{l_{2}}\right\rceil} i^{1-c_{2}}}{\sum_{i=1}^{\left\lceil n^{l_{2}}\right\rceil} i^{-c_{2}}}-\frac{1}{\left(2-c_{2}\right) z\left(c_{2}\right)} & <\frac{1}{\left(2-c_{2}\right) z\left(c_{2}\right)}\left(\frac{n^{\kappa-1}\left\lceil n^{l_{2}}\right\rceil^{2-c_{2}}}{1-\left\lceil n^{l_{2}}\right\rceil^{1-c_{2}}}-1\right) \\
& =\frac{n^{\kappa-1}\left\lceil n^{l_{2}}\right\rceil^{2-c_{2}}-1+\left\lceil n^{l_{2}}\right\rceil^{1-c_{2}}}{\left(2-c_{2}\right) z\left(c_{2}\right)\left(1-\left\lceil n^{l_{2}}\right\rceil^{1-c_{2}}\right)}
\end{aligned}
$$

To further bound (120), we first note

$$
\begin{gathered}
\left\lceil n^{l_{2}}\right\rceil^{1-c_{2}}<n^{l_{2}\left(1-c_{2}\right)}, \\
\left\lceil n^{l_{2}}\right\rceil^{2-c_{2}}<(n+1)^{l_{2}\left(2-c_{2}\right)}<n^{l_{2}\left(2-c_{2}\right)}+1,
\end{gathered}
$$

where (121) holds since $c_{2}>1$ (so that $x^{1-c_{2}}$ is strictly decreasing for $x>0$ ), and the first inequality in (122) holds since $c_{2}<2$ (so that $x^{2-c_{2}}$ is strictly increasing for $x>0$ ). The second inequality in (122) holds since $l_{2}\left(2-c_{2}\right) \in(0,1)$, and for any $c \in(0,1)$,

$$
\begin{aligned}
(n+1)^{c} & =c \int_{y=0}^{n+1} y^{c-1} d y=c \int_{y=0}^{n} y^{c-1} d y+c \int_{y=n}^{n+1} y^{c-1} d y \\
& =n^{c}+c \int_{y=0}^{1}(y+n)^{c-1} d y \leq n^{c}+c \int_{y=0}^{1} y^{c-1} d y=n^{c}+1,
\end{aligned}
$$

where the inequality is $(y+n)^{c-1}<y^{c-1} \forall y>0$, which holds since $c \in(0,1)$. Combining (120), (122), and (122), and recalling $l_{2}\left(2-c_{2}\right)=1-\kappa$ by assumption, we obtain

$$
n^{\kappa-1} \frac{\sum_{i=1}^{\left\lceil l_{2}\right\rceil} i^{1-c_{2}}}{\sum_{i=1}^{\left\lceil n^{l_{2}}\right\rceil} i^{-c_{2}}}-\frac{1}{\left(2-c_{2}\right) z\left(c_{2}\right)}<\frac{n^{\kappa-1}+n^{l_{2}\left(1-c_{2}\right)}}{\left(2-c_{2}\right) z\left(c_{2}\right)\left(1-\left\lceil n^{l_{2}}\right\rceil^{1-c_{2}}\right)}<\frac{n^{\kappa-1}+n^{l_{2}\left(1-c_{2}\right)}}{\left(2-c_{2}\right) z\left(c_{2}\right) / 2},
$$

where the second inequality holds for sufficiently large $n$, because $\left\lceil n^{l_{2}}\right\rceil^{1-c_{2}}$ decays to zero as $n \rightarrow \infty$, so $\left\lceil n^{l_{2}}\right\rceil^{1-c_{2}}<\frac{1}{2}$ for large enough $n$. For the lower bound of (118), we first use Lemma 17 and $\sum_{i=1}^{\left\lceil n^{l_{2}}\right\rceil} i^{-c_{2}}<z\left(c_{2}\right)$ to obtain

$$
\begin{aligned}
n^{\kappa-1} \frac{\sum_{i=1}^{\left\lceil n^{l_{2}}\right\rceil} i^{1-c_{2}}}{\sum_{i=1}^{\left\lceil n_{2}\right\rceil} i^{-c_{2}}}-\frac{1}{\left(2-c_{2}\right) z\left(c_{2}\right)} & >n^{\kappa-1} \frac{\left(\left\lceil n^{l_{2}}\right\rceil+1\right)^{2-c_{2}}-1}{\left(2-c_{2}\right) z\left(c_{2}\right)}-\frac{1}{\left(2-c_{2}\right) z\left(c_{2}\right)} \\
& =-\frac{n^{\kappa-1}}{\left(2-c_{2}\right) z\left(c_{2}\right)}+\frac{n^{\kappa-1}\left(\left\lceil n^{l_{2}}\right\rceil+1\right)^{2-c_{2}}-1}{\left(2-c_{2}\right) z\left(c_{2}\right)} .
\end{aligned}
$$

Now since $c_{2}<2, x^{2-c_{2}}$ is strictly increasing for $x>0$, so $\left(\left\lceil n^{l_{2}}\right\rceil+1\right)^{2-c_{2}}>n^{l_{2}\left(2-c_{2}\right)}$. Since also $l_{2}\left(2-c_{2}\right)=1-\kappa$ by assumption, $n^{\kappa-1}\left(\left\lceil n^{l_{2}}\right\rceil+1\right)^{2-c_{2}}>1$; together with (124), we obtain

$$
n^{\kappa-1} \frac{\sum_{i=1}^{\left\lceil n^{l_{2}}\right\rceil} i^{1-c_{2}}}{\sum_{i=1}^{\left\lceil n_{2}\right\rceil} i^{-c_{2}}}-\frac{1}{\left(2-c_{2}\right) z\left(c_{2}\right)}>-\frac{n^{\kappa-1}}{\left(2-c_{2}\right) z\left(c_{2}\right)} .
$$

Finally, (123) and (125) together imply

$$
\left|n^{\kappa-1} \frac{\sum_{i=1}^{\left\lceil n^{l_{2}}\right\rceil} i^{1-c_{2}}}{\sum_{i=1}^{\left\lceil n^{l_{2}}\right\rceil} i^{-c_{2}}}-\frac{1}{\left(2-c_{2}\right) z\left(c_{2}\right)}\right|=O\left(n^{-\min \left\{l_{2}\left(c_{2}-1\right), 1-\kappa\right\}}\right) .
$$

Combining (119) and (126), we have shown

$$
\left|\mathbb{E}\left[N_{v}\right]-\eta_{1}\right|=O\left(n^{-\min \left\{l_{1}\left(c_{1}-2\right), l_{2}\left(c_{2}-1\right), 1-\kappa\right\}}\right) .
$$


Hence, by assumption $\gamma<\min \left\{l_{1}\left(c_{1}-2\right), l_{2}\left(c_{2}-1\right), 1-\kappa\right\}$, we have $\left|\mathbb{E}\left[N_{v}\right]-\eta_{1}\right| \leq n^{-\gamma} / 2$ for sufficiently large $n$. For such $n$, we can then use the triangle and Chebyshev inequalities to write

$$
\begin{aligned}
\mathbb{P}\left[\left|\frac{1}{n} \sum_{v \in V_{n}} N_{v}-\eta_{1}\right|>n^{-\gamma}\right] & \leq \mathbb{P}\left[\left|\frac{1}{n} \sum_{v \in V_{n}} N_{v}-\mathbb{E}\left[N_{v}\right]\right|>n^{-\gamma} / 2\right] \leq 4 n^{2 \gamma} \operatorname{Var}\left(\frac{1}{n} \sum_{v \in V_{n}} N_{v}\right) \\
& =4 n^{2 \gamma-1} \operatorname{Var}\left(N_{v}\right) \leq 4 n^{2 \gamma-1} \mathbb{E}\left[N_{v}^{2}\right]=O\left(n^{l_{2}+2 \gamma-1}\right),
\end{aligned}
$$

where the first equality holds since $\left\{N_{v}\right\}_{v \in V_{n}}$ are identically distributed and the second follows since

$$
\begin{aligned}
& \mathbb{E}\left[N_{v}^{2}\right]=\left(1-n^{\kappa-1}\right) \mathbb{E}\left[N_{v}^{2} \mid U_{v}=1\right]+n^{\kappa-1} \mathbb{E}\left[N_{v}^{2} \mid U_{v}=0\right] \\
& =\left(1-n^{\kappa-1}\right) \frac{\sum_{i=1}^{\left\lceil n^{l_{1}}\right\rceil} i^{2-c_{1}}}{\sum_{i=1}^{\left\lceil n_{1}^{l_{1}}\right\rceil} i^{-c_{1}}}+n^{\kappa-1} \frac{\sum_{i=1}^{\left\lceil n^{l_{2}}\right\rceil} i^{2-c_{2}}}{\sum_{i=1}^{\left\lceil n^{l_{1}}\right\rceil} i^{-c_{2}}}=O\left(n^{l_{2}}\right),
\end{aligned}
$$

where the final step uses $n^{\kappa-1} \sum_{i=1}^{\left\lceil n^{l_{2}}\right\rceil} i^{2-c_{2}}=O\left(n^{l_{2}}\right)$ by Lemma 17 and the assumption $l_{2}\left(2-c_{2}\right)=1-\kappa$ (the other terms are $\Theta(1)$ ).

Lemma 20. Let $\eta_{2}=\eta_{1}^{2}$, where $\eta_{1}$ is defined in Lemma 19. Then if $\gamma<\min \left\{l_{1}\left(c_{1}-2\right), l_{2}\left(c_{2}-1\right), 1-\right.$ $\left.\kappa, 1-l_{2}\right\}$, we have

$$
\mathbb{P}\left[\left|\frac{1}{n} \sum_{v \in V_{n}} N_{v} D_{v}-\eta_{2}\right|>n^{-\gamma}\right]=O\left(n^{l_{2}+2 \gamma-1}\right) .
$$

Proof. We begin by bounding $\left|\mathbb{E}\left[N_{v} D_{v}\right]-\eta_{1}^{2}\right|$. For this, first note that since $D_{v}=D_{v}^{\prime}+1$, with $D_{v}^{\prime} \sim \operatorname{Binomial}\left(\sum_{u \in V_{n}} N_{u}-n, \frac{1}{n}\right)$,

$$
\begin{aligned}
\mathbb{E}\left[N_{v} D_{v}\right] & =\mathbb{E}\left[N_{v}\right]+\mathbb{E}\left[N_{v} \mathbb{E}\left[D_{v}^{\prime} \mid\left\{N_{u}\right\}_{u \in V_{n}}\right]\right]=\mathbb{E}\left[N_{v}\right]+\mathbb{E}\left[N_{v} \frac{1}{n}\left(\sum_{u \in V_{n}} N_{u}-n\right)\right] \\
& =\frac{1}{n} \sum_{u \in V_{n}} \mathbb{E}\left[N_{v} N_{u}\right]=\frac{n-1}{n}\left(\mathbb{E}\left[N_{v}\right]\right)^{2}+\frac{1}{n} \mathbb{E}\left[N_{v}^{2}\right] .
\end{aligned}
$$

We can then write

$$
\begin{aligned}
\left|\mathbb{E}\left[N_{v} D_{v}\right]-\eta_{1}^{2}\right| & =\left|\frac{n-1}{n}\left(\left(\mathbb{E}\left[N_{v}\right]\right)^{2}-\eta_{1}^{2}\right)+\frac{1}{n} \mathbb{E}\left[N_{v}^{2}\right]-\frac{1}{n} \eta_{1}^{2}\right| \\
& <\left|\left(\mathbb{E}\left[N_{v}\right]\right)^{2}-\eta_{1}^{2}\right|+\frac{1}{n} \mathbb{E}\left[N_{v}^{2}\right]+\frac{1}{n} \eta_{1}^{2}=\left|\left(\mathbb{E}\left[N_{v}\right]\right)^{2}-\eta_{1}^{2}\right|+O\left(n^{l_{2}-1}\right),
\end{aligned}
$$

where the final inequality follows from (128) and the fact that $\eta_{1}$ is $O(1)$. Furthermore, it is straightforward to verify

$$
\left(\mathbb{E}\left[N_{v}\right]\right)^{2}-\eta_{1}^{2}=\left(\mathbb{E}\left[N_{v}\right]-\eta_{1}\right)^{2}+2 \eta_{1}\left(\mathbb{E}\left[N_{v}\right]-\eta_{1}\right) .
$$

Combining the previous two equations, we obtain

$$
\begin{aligned}
\left|\mathbb{E}\left[N_{v} D_{v}\right]-\eta_{1}^{2}\right| & \leq\left|\mathbb{E}\left[N_{v}\right]-\eta_{1}\right|^{2}+2 \eta_{1}\left|\mathbb{E}\left[N_{v}\right]-\eta_{1}\right|+O\left(n^{l_{2}-1}\right) \\
& =O\left(\left|\mathbb{E}\left[N_{v}\right]-\eta_{1}\right|+n^{l_{2}-1}\right)=O\left(n^{-\min \left\{l_{1}\left(c_{1}-2\right), l_{2}\left(c_{2}-1\right), 1-\kappa, 1-l_{2}\right\}}\right),
\end{aligned}
$$

where the first equality uses the fact that $\left|\mathbb{E}\left[N_{v}\right]-\eta_{1}\right| \rightarrow 0$ by (127), and the quadratic term $\left|\mathbb{E}\left[N_{v}\right]-\eta_{1}\right|^{2}$ will converge to zero more quickly than the linear term $\left|\mathbb{E}\left[N_{v}\right]-\eta_{1}\right|$; the second 
equality is by (127). Now by assumption $\gamma<\min \left\{l_{1}\left(c_{1}-2\right), l_{2}\left(c_{2}-1\right), 1-\kappa, 1-l_{2}\right\}$, we have $\left|\mathbb{E}\left[N_{v} D_{v}\right]-\eta_{1}^{2}\right| \leq n^{-\gamma} / 2$ for large enough $n$; for such $n$,

$$
\begin{aligned}
\mathbb{P}\left[\left|\frac{1}{n} \sum_{v \in V_{n}} N_{v} D_{v}-\eta_{2}\right|>n^{-\gamma}\right] \leq \mathbb{P}\left[\left|\frac{1}{n} \sum_{v \in V_{n}} N_{v} D_{v}-\mathbb{E}\left[N_{v} D_{v}\right]\right|>n^{-\gamma} / 2\right] \\
\leq 4 n^{2 \gamma} \operatorname{Var}\left(\frac{1}{n} \sum_{v \in V_{n}} N_{v} D_{v}\right)=O\left(n^{2 \gamma-1} \operatorname{Var}\left[N_{v} D_{v}\right]+n^{2 \gamma} \operatorname{Cov}\left(N_{v} D_{v}, N_{u} D_{u}\right)\right),
\end{aligned}
$$

where we used Chebyshev's inequality and $\left\{N_{v} D_{v}\right\}_{v \in V_{n}}$ being identically distributed (note we assume $v \neq u$ in (130)).

It remains to bound $\operatorname{Var}\left[N_{v} D_{v}\right]$ and $\operatorname{Cov}\left(N_{v} D_{v}, N_{u} D_{u}\right)$. First, we bound the variance of $N_{v} D_{v}$ by its second moment, which is

$$
\mathbb{E}\left[N_{v}^{2} D_{v}^{2}\right]=\mathbb{E}\left[N_{v}^{2}\left(D_{v}^{\prime}\right)^{2}\right]+2 \mathbb{E}\left[N_{v}^{2} D_{v}^{\prime}\right]+\mathbb{E}\left[N_{v}^{2}\right] .
$$

By $D_{v}^{\prime} \sim \operatorname{Binomial}\left(\sum_{u \in V_{n}} N_{u}-n, \frac{1}{n}\right)$, we have for the second term

$$
\mathbb{E}\left[N_{v}^{2} D_{v}^{\prime}\right]=\mathbb{E}\left[N_{v}^{2} \frac{1}{n}\left(\sum_{u \in V_{n}} N_{u}-n\right)\right]<\frac{1}{n} \mathbb{E}\left[N_{v}^{3}\right]+\mathbb{E}\left[N_{v}^{2}\right] \mathbb{E}\left[N_{v}\right],
$$

where for the equality we took conditional expectation with respect to $\left\{N_{u}\right\}_{u \in V_{n}}$ and the inequality dropped $-\mathbb{E}\left[N_{v}^{2}\right]$. Then by (131),

$$
\mathbb{E}\left[N_{v}^{2} D_{v}^{2}\right]<\mathbb{E}\left[N_{v}^{2}\left(D_{v}^{\prime}\right)^{2}\right]+\frac{2}{n} \mathbb{E}\left[N_{v}^{3}\right]+\mathbb{E}\left[N_{v}^{2}\right]\left(2 \mathbb{E}\left[N_{v}\right]+1\right) .
$$

Again using $D_{v}^{\prime} \sim \operatorname{Binomial}\left(\sum_{u \in V_{n}} N_{u}-n, \frac{1}{n}\right)$, and again taking conditional expectation with respect to $\left\{N_{u}\right\}_{u \in V_{n}}$, we have

$$
\mathbb{E}\left[N_{v}^{2}\left(D_{v}^{\prime}\right)^{2}\right]=\mathbb{E}\left[N_{v}^{2} \frac{1}{n} \frac{n-1}{n}\left(\sum_{u \in V_{n}} N_{u}-n\right)\right]+\mathbb{E}\left[N_{v}^{2} \frac{1}{n^{2}}\left(\sum_{u \in V_{n}} N_{u}-n\right)^{2}\right],
$$

so we can obtain the bound

$$
\begin{aligned}
\mathbb{E}\left[N_{v}^{2}\left(D_{v}^{\prime}\right)^{2}\right] & <\frac{1}{n} \mathbb{E}\left[N_{v}^{2} \sum_{u \in V_{n}} N_{u}\right]+\frac{1}{n^{2}} \mathbb{E}\left[N_{v}^{2}\left(\sum_{u \in V_{n}} N_{u}\right)^{2}\right]-\frac{2}{n} \mathbb{E}\left[N_{v}^{2} \sum_{u \in V_{n}} N_{u}\right]+\mathbb{E}\left[N_{v}^{2}\right] \\
& <\frac{1}{n^{2}} \mathbb{E}\left[N_{v}^{2}\left(\sum_{u \in V_{n}} N_{u}\right)^{2}\right]+\mathbb{E}\left[N_{v}^{2}\right],
\end{aligned}
$$

where for the first inequality we bounded $\frac{n-1}{n}$ by 1 and dropped $-\frac{(n-1)}{n} \mathbb{E}\left[N_{v}^{2}\right]$, and for the second we dropped $-\frac{1}{n} \mathbb{E}\left[N_{v}^{2} \sum_{u \in V_{n}} N_{u}\right]$. Next, rewriting the term in the first expectation in (133) as

$$
\begin{aligned}
\left(\sum_{u \in V_{n}} N_{u}\right)^{2} & =N_{v}^{2}+2 N_{v} \sum_{u \in V_{n} \backslash\{v\}} N_{u}+\left(\sum_{u \in V_{n} \backslash\{v\}} N_{u}\right)^{2} \\
& =N_{v}^{2}+2 N_{v} \sum_{u \in V_{n} \backslash\{v\}} N_{u}+\sum_{u \in V_{n} \backslash\{v\}} N_{u}^{2}+\sum_{u, w \in V_{n} \backslash\{v\}: u \neq w} N_{u} N_{w}
\end{aligned}
$$


it is straightforward to see

$$
\mathbb{E}\left[N_{v}^{2}\left(\sum_{u \in V_{n}} N_{u}\right)^{2}\right]<\mathbb{E}\left[N_{v}^{4}\right]+2 n \mathbb{E}\left[N_{v}^{3}\right] \mathbb{E}\left[N_{v}\right]+n\left(\mathbb{E}\left[N_{v}^{2}\right]\right)^{2}+n^{2} \mathbb{E}\left[N_{v}^{2}\right]\left(\mathbb{E}\left[N_{v}\right]\right)^{2} .
$$

Substituting the previous equation into (133), we then obtain

$$
\mathbb{E}\left[N_{v}^{2}\left(D_{v}^{\prime}\right)^{2}\right]<\frac{1}{n^{2}} \mathbb{E}\left[N_{v}^{4}\right]+\frac{2}{n} \mathbb{E}\left[N_{v}^{3}\right] \mathbb{E}\left[N_{v}\right]+\frac{1}{n}\left(\mathbb{E}\left[N_{v}^{2}\right]\right)^{2}+\mathbb{E}\left[N_{v}^{2}\right]\left(\mathbb{E}\left[N_{v}\right]\right)^{2}+\mathbb{E}\left[N_{v}^{2}\right] .
$$

Next, combine (132) with (134) and recall $\mathbb{E}\left[N_{v}\right]=O(1)$ to obtain

$$
\mathbb{E}\left[N_{v}^{2} D_{v}^{2}\right]=O\left(\frac{1}{n^{2}} \mathbb{E}\left[N_{v}^{4}\right]+\frac{1}{n} \mathbb{E}\left[N_{v}^{3}\right]+\frac{1}{n}\left(\mathbb{E}\left[N_{v}^{2}\right]\right)^{2}+\mathbb{E}\left[N_{v}^{2}\right]\right) .
$$

Furthermore, we can use Lemma 17 to write

$$
\begin{aligned}
& \mathbb{E}\left[N_{v}^{3}\right]=\left(1-n^{\kappa-1}\right) \frac{\left.\sum_{i=1}^{\left\lceil n_{1}\right.}\right\rceil i^{3-c_{1}}}{\sum_{i=1}^{\left\lceil n_{1} l_{1}\right.} i^{-c_{1}}}+n^{\kappa-1} \frac{\sum_{i=1}^{\left\lceil n^{l_{2}}\right\rceil} i^{3-c_{2}}}{\sum_{i=1}^{\left\lceil n_{1}^{l_{1}}\right\rceil} i^{-c_{2}}} \\
& =O\left(n^{l_{1}\left(4-c_{1}\right)}+n^{l_{2}\left(4-c_{2}\right)+\kappa-1}\right)=O\left(n^{l_{1}\left(4-c_{1}\right)}+n^{2 l_{2}}\right) \text {, } \\
& \mathbb{E}\left[N_{v}^{4}\right]=\left(1-n^{\kappa-1}\right) \frac{\sum_{i=1}^{\left\lceil n^{l_{1}}\right\rceil} i^{4-c_{1}}}{\sum_{i=1}^{\left\lceil n_{1}^{l_{1}}\right\rceil} i^{-c_{1}}}+n^{\kappa-1} \frac{\sum_{i=1}^{\left\lceil n^{l_{2}}\right\rceil} i^{4-c_{2}}}{\sum_{i=1}^{\left\lceil n^{l_{1}}\right\rceil} i^{-c_{2}}} \\
& =O\left(n^{l_{1}\left(5-c_{1}\right)}+n^{l_{2}\left(5-c_{2}\right)+\kappa-1}\right)=O\left(n^{l_{1}\left(5-c_{1}\right)}+n^{3 l_{2}}\right) \text {. }
\end{aligned}
$$

where we have also used $l_{2}\left(2-c_{2}\right)=1-\kappa$. Using these bounds, as well as (128), we can bound (135) as

$$
\mathbb{E}\left[N_{v}^{2} D_{v}^{2}\right]=O\left(n^{l_{1}\left(5-c_{1}\right)-2}+n^{3 l_{2}-2}+n^{l_{1}\left(4-c_{1}\right)-1}+n^{2 l_{2}-1}+n^{l_{2}}\right) .
$$

Finally, since $l_{1}<1$ and $c_{1}>3, n^{l_{1}\left(5-c_{1}\right)-2}$ and $n^{l_{1}\left(4-c_{1}\right)-1}$ decay with $n$, and since $l_{2}<1, n^{3 l_{2}-2}$ and $n^{2 l_{2}-1}$ are bounded by $n^{l_{2}}$. Thus,

$$
\mathbb{E}\left[N_{v}^{2} D_{v}^{2}\right]=O\left(n^{l_{2}}\right)
$$

We now turn to $\operatorname{Cov}\left(N_{v} D_{v}, N_{u} D_{u}\right)$. We first use the fact that $N_{v}, N_{u}$ and $D_{v}^{\prime}, D_{u}^{\prime}$ are each identically distributed to write

$$
\mathbb{E}\left[N_{v} N_{u} D_{v} D_{u}\right]=\mathbb{E}\left[N_{v} N_{u} D_{v}^{\prime} D_{u}^{\prime}\right]+2 \mathbb{E}\left[N_{v} N_{u} D_{v}^{\prime}\right]+\left(\mathbb{E}\left[N_{v}\right]\right)^{2}
$$

For the first term in (138), we observe

$$
\begin{aligned}
\mathbb{E}\left[N_{v} N_{u} D_{v}^{\prime} D_{u}^{\prime}\right] & =\mathbb{E}\left[N_{v} N_{u} D_{v}^{\prime} \mathbb{E}\left[D_{u}^{\prime} \mid\left\{N_{w}\right\}_{w \in V_{n}}, D_{v}^{\prime}\right]\right]=\mathbb{E}\left[N_{v} N_{u} D_{v}^{\prime} \frac{1}{n-1}\left(\sum_{w \in V_{n}} N_{w}-n-D_{v}^{\prime}\right)\right] \\
& <\frac{1}{n-1} \mathbb{E}\left[N_{v} N_{u} D_{v}^{\prime} \sum_{w \in V_{n}} N_{w}\right]-\mathbb{E}\left[N_{v} N_{u} D_{v}^{\prime}\right]
\end{aligned}
$$

where we used $D_{u}^{\prime} \sim \operatorname{Binomial}\left(\sum_{w \in V_{n}} N_{w}-n-D_{v}^{\prime}, \frac{1}{n-1}\right)$ in the second line, and used $\frac{n}{n-1}>1$ and dropped a negative term in the third line; substituting into (138) gives

$$
\mathbb{E}\left[N_{v} N_{u} D_{v} D_{u}\right]<\frac{1}{n-1} \mathbb{E}\left[N_{v} N_{u} D_{v}^{\prime} \sum_{w \in V_{n}} N_{w}\right]+\mathbb{E}\left[N_{v} N_{u} D_{v}^{\prime}\right]+\left(\mathbb{E}\left[N_{v}\right]\right)^{2} .
$$


Now for the first summand in (139), we take conditional expectation with respect to $\left\{N_{w^{\prime}}\right\}_{w^{\prime} \in V_{n}}$ to obtain

$$
\begin{aligned}
\mathbb{E}\left[N_{v} N_{u} D_{v}^{\prime} \sum_{w \in V_{n}} N_{w}\right] & =\mathbb{E}\left[N_{v} N_{u} \sum_{w \in V_{n}} N_{w} \frac{1}{n}\left(\sum_{w^{\prime} \in V_{n}} N_{w^{\prime}}-n\right)\right] \\
& =\frac{1}{n} \mathbb{E}\left[N_{v} N_{u}\left(\sum_{w \in V_{n}} N_{w}\right)^{2}\right]-\mathbb{E}\left[N_{v} N_{u} \sum_{w \in V_{n}} N_{w}\right],
\end{aligned}
$$

while for the second summand in (139), we take conditional expectation with respect to $\left\{N_{w}\right\}_{w \in V_{n}}$ to reach

$$
\mathbb{E}\left[N_{v} N_{u} D_{v}^{\prime}\right]=\mathbb{E}\left[N_{v} N_{u} \frac{1}{n}\left(\sum_{w \in V_{n}} N_{w}-n\right)\right]=\frac{1}{n} \mathbb{E}\left[N_{v} N_{u} \sum_{w \in V_{n}} N_{w}\right]-\left(\mathbb{E}\left[N_{v}\right]\right)^{2} .
$$

Combining (139), (140), and (141), we obtain

$$
\mathbb{E}\left[N_{v} N_{u} D_{v} D_{u}\right]<\frac{1}{n(n-1)} \mathbb{E}\left[N_{v} N_{u}\left(\sum_{w \in V_{n}} N_{w}\right)^{2}\right],
$$

where $\left(\mathbb{E}\left[N_{v}\right]\right)^{2}$ and $-\left(\mathbb{E}\left[N_{v}\right]\right)^{2}$ have canceled, and where we have dropped the negative term $\left(\frac{1}{n}-\frac{1}{n-1}\right) \mathbb{E}\left[N_{v} N_{u} \sum_{w \in V_{n}} N_{w}\right]$. Next, rewriting the squared term in (142) as

$$
\begin{aligned}
\left(\sum_{w \in V_{n}} N_{w}\right)^{2}= & N_{v}^{2}+N_{u}^{2}+2 N_{v} N_{u}+2 N_{v} \sum_{w \in V_{n} \backslash\{v, u\}} N_{w} \\
& +2 N_{u} \sum_{w \in V_{n} \backslash\{v, u\}} N_{w}+\sum_{w \in V_{n} \backslash\{v, u\}} N_{w}^{2}+\sum_{w, w^{\prime} \in V_{n} \backslash\{v, u\}: w \neq w^{\prime}} N_{w} N_{w^{\prime}}
\end{aligned}
$$

it is straightforward to verify

$$
\begin{aligned}
\mathbb{E}\left[N_{v} N_{u}\left(\sum_{w \in V_{n}} N_{w}\right)^{2}\right]< & 2 \mathbb{E}\left[N_{v}^{3}\right] \mathbb{E}\left[N_{v}\right]+2\left(\mathbb{E}\left[N_{v}^{2}\right]\right)^{2} \\
& +5 n \mathbb{E}\left[N_{v}^{2}\right]\left(\mathbb{E}\left[N_{v}\right]\right)^{2}+(n-2)(n-3)\left(\mathbb{E}\left[N_{v}\right]\right)^{4} .
\end{aligned}
$$

We have therefore shown

$$
\begin{aligned}
\mathbb{E}\left[N_{v} N_{u} D_{v} D_{u}\right]<\frac{(n-2)(n-3)\left(\mathbb{E}\left[N_{v}\right]\right)^{4}}{n(n-1)} & \\
& +\frac{2 \mathbb{E}\left[N_{v}^{3}\right] \mathbb{E}\left[N_{v}\right]+2\left(\mathbb{E}\left[N_{v}^{2}\right]\right)^{2}+5 n \mathbb{E}\left[N_{v}^{2}\right]\left(\mathbb{E}\left[N_{v}\right]\right)^{2}}{n(n-1)} .
\end{aligned}
$$

Furthermore, we have

$$
\left(\mathbb{E}\left[N_{v} D_{v}\right]\right)^{2}>\left(\frac{n-1}{n}\right)^{2}\left(\mathbb{E}\left[N_{v}\right]\right)^{4}>\frac{(n-2)(n-3)\left(\mathbb{E}\left[N_{v}\right]\right)^{4}}{n(n-1)},
$$

where the first inequality is by (129) and the second uses $n-1>n-2$ and $\frac{n-1}{n}>\frac{n-3}{n-1}$, the latter of which can be seen by observing

$$
n>0 \Rightarrow 1-2 n>-3 n \Rightarrow(n-1)^{2}=n^{2}-2 n+1>n^{2}-3 n=n(n-3) .
$$


We therefore obtain

$$
\begin{aligned}
\operatorname{Cov}\left(N_{v} D_{v}, N_{u} D_{u}\right) & =\mathbb{E}\left[N_{v} N_{u} D_{v} D_{u}\right]-\left(\mathbb{E}\left[N_{v} D_{v}\right]\right)^{2} \\
& <\frac{2 \mathbb{E}\left[N_{v}^{3}\right] \mathbb{E}\left[N_{v}\right]+2\left(\mathbb{E}\left[N_{v}^{2}\right]\right)^{2}+5 n \mathbb{E}\left[N_{v}^{2}\right]\left(\mathbb{E}\left[N_{v}\right]\right)^{2}}{n(n-1)} .
\end{aligned}
$$

Additionally, since $\mathbb{E}\left[N_{v}\right]=O(1), \mathbb{E}\left[N_{v}^{2}\right]=O\left(n^{l_{2}}\right)$ (by (128)), and $\mathbb{E}\left[N_{v}^{3}\right]=O\left(n^{l_{1}\left(4-c_{1}\right)}+n^{2 l_{2}}\right)$ (by (136)), we have

$$
\operatorname{Cov}\left(N_{v} D_{v}, N_{u} D_{u}\right)=O\left(n^{l_{1}\left(4-c_{1}\right)-2}+n^{2 l_{2}-2}+n^{l_{2}-1}\right) .
$$

Finally, we note $n^{2 l_{2}-2}$ decays more quickly than $n^{l_{2}-1}$, and $l_{2}-1>-1>l_{1}\left(4-c_{1}\right)-2$ by assumption $l_{1}, l_{2} \in(0,1), c_{1}>3$. Hence, the $n^{l_{2}-1}$ dominates above, i.e. $\operatorname{Cov}\left(N_{v} D_{v}, N_{u} D_{u}\right)=$ $O\left(n^{l_{2}-1}\right)$. Combining this observation with (130) and (137), we obtain

$$
\mathbb{P}\left[\left|\frac{1}{n} \sum_{v \in V_{n}} N_{v} D_{v}-\eta_{2}\right|>n^{-\gamma}\right]=O\left(n^{l_{2}+2 \gamma-1}\right),
$$

which completes the proof.

Lemma 21. Let $\eta_{3}=z\left(c_{1}-2\right) / z\left(c_{1}\right)$, which is well-defined by assumption $c_{1}>3$. Then if $\gamma \in$ $\left(0, \min \left\{1-\kappa, l_{1}\left(c_{1}-3\right)\right\}\right)$, we have

$$
\mathbb{P}\left[\left|\frac{1}{n} \sum_{v \in V_{n}} U_{v} N_{v}^{2}-\eta_{3}\right|>n^{-\gamma}\right]=O\left(n^{l_{1}\left(5-c_{1}\right)+2 \gamma-1}\right) .
$$

Proof. First, for $v \in V_{n}$, we have by definition

$$
\eta_{3}-\mathbb{E}\left[U_{v} N_{v}^{2}\right]=\frac{z\left(c_{1}-2\right)}{z\left(c_{1}\right)}-\left(1-n^{\kappa-1}\right) \frac{\sum_{i=1}^{\left\lceil n^{l_{1}}\right\rceil} i^{2-c_{1}}}{\sum_{i=1}^{\left\lceil n^{l_{1}}\right\rceil} i^{-c_{1}}} .
$$

By Lemma 15, this is strictly positive. We also have

$$
\begin{aligned}
\eta_{3} & -\mathbb{E}\left[U_{v} N_{v}^{2}\right]=n^{\kappa-1} \eta_{3}-\left(1-n^{\kappa-1}\right)\left(\frac{\sum_{i=1}^{\left\lceil n^{l_{1}}\right\rceil} i^{2-c_{1}}}{\left.\sum_{i=1}^{\left\lceil n_{1}\right.}\right\rceil i^{-c_{1}}}-\frac{z\left(c_{1}-2\right)}{z\left(c_{1}\right)}\right) \\
& <n^{\kappa-1} \eta_{3}-\frac{\left(1-n^{\kappa-1}\right)\left(\sum_{i=1}^{\left\lceil n^{l_{1}}\right\rceil} i^{2-c_{1}}-z\left(c_{1}-2\right)\right)}{z\left(c_{1}\right)}=n^{\kappa-1} \eta_{3}+\frac{\left(1-n^{\kappa-1}\right) \sum_{i=\left\lceil n^{\left.l_{1}\right\rceil+1}\right.}^{\infty} i^{2-c_{1}}}{z\left(c_{1}\right)} \\
& <n^{\kappa-1} \eta_{3}+\frac{\left(1-n^{\kappa-1}\right) z\left(c_{1}-2\right)\left\lceil n^{l_{1}}\right\rceil^{3-c_{1}}}{z\left(c_{1}\right)}=O\left(n^{-\min \left\{1-\kappa, l_{1}\left(c_{1}-3\right)\right\}}\right),
\end{aligned}
$$

where the second inequality uses Lemma 16 . Now by assumption $\gamma<\min \left\{1-\kappa, l_{1}\left(c_{1}-3\right)\right\}$, we have $\left|\mathbb{E}\left[U_{v} N_{v}^{2}\right]-\eta_{3}\right| \leq n^{-\gamma} / 2$ for large $n$; for such $n$, by Chebyshev's inequality,

$$
\mathbb{P}\left[\left|\frac{1}{n} \sum_{v \in V_{n}} U_{v} N_{v}^{2}-\eta_{3}\right|>n^{-\gamma}\right] \leq \mathbb{P}\left[\left|\frac{1}{n} \sum_{v \in V_{n}} U_{v} N_{v}^{2}-\mathbb{E}\left[U_{v} N_{v}^{2}\right]\right|>n^{-\gamma} / 2\right] \leq 4 n^{2 \gamma-1} \mathbb{E}\left[U_{v}^{2} N_{v}^{4}\right] .
$$

To bound $\mathbb{E}\left[U_{v}^{2} N_{v}^{4}\right]$, first note $U_{v}^{2}=U_{v}$, and

$$
\mathbb{E}\left[U_{v} N_{v}^{4}\right]=\left(1-n^{\kappa-1}\right) \frac{\sum_{i=1}^{\left\lceil n^{l_{1}}\right\rceil} i^{4-c_{1}}}{\sum_{i=1}^{\left\lceil n^{l_{1}}\right\rceil} i^{-c_{1}}}<\sum_{i=1}^{\left\lceil n^{l_{1}}\right\rceil} i^{4-c_{1}} .
$$


Since $c_{1}<4$, we can use Lemma 17 to write

$$
\sum_{i=1}^{\left\lceil n^{l_{1}}\right\rceil} i^{4-c_{1}}=O\left(n^{l_{1}\left(5-c_{1}\right)}\right)
$$

so we conclude

$$
\mathbb{P}\left[\left|\frac{1}{n} \sum_{v \in V_{n}} U_{v} N_{v}^{2}-\eta_{3}\right|>n^{-\gamma}\right]=O\left(n^{l_{1}\left(5-c_{1}\right)+2 \gamma-1}\right),
$$

which completes the proof.

Lemma 22. Let $\zeta^{*}=\eta_{1}$, with $\eta_{1}$ defined in Lemma 19. Then if $\gamma \in\left(0, \min \left\{l_{1}\left(c_{1}-2\right), l_{2}\left(c_{2}-1\right), 1-\kappa\right\}\right)$, we have

$$
\mathbb{P}\left[\left|\frac{\sum_{v \in V_{n}} U_{v} D_{v}}{\sum_{v \in V_{n}} U_{v}}-\zeta^{*}\right|>n^{-\gamma}\right]=O\left(n^{2 \gamma-\min \{\kappa / 3,1-\kappa\}}\right) .
$$

Proof. We first recall that $\sum_{v \in V_{n}} U_{v} D_{v} / \sum_{v \in V_{n}} U_{v}=1$ whenever $\sum_{v \in V_{n}} U_{v}=0$ by convention. We also define the event $E=\left\{\sum_{v \in V_{n}} U_{v}>0\right\}$. Note that, since $\sum_{v \in V_{n}} U_{v} \sim \operatorname{Binomial}\left(n, 1-n^{\kappa-1}\right)$, we have $\mathbb{P}\left[E^{C}\right]=\left(n^{\kappa-1}\right)^{n}$. This implies

$$
\mathbb{P}\left[\left|\frac{\sum_{v \in V_{n}} U_{v} D_{v}}{\sum_{v \in V_{n}} U_{v}}-\zeta^{*}\right|>n^{-\gamma}\right] \leq \mathbb{P}\left[\left|\frac{\sum_{v \in V_{n}} U_{v} D_{v}}{\sum_{v \in V_{n}} U_{v}}-\zeta^{*}\right|>n^{-\gamma} \mid E\right]+n^{n(\kappa-1)} .
$$

The remainder of the proof will involve bounding the first term in (143). To this end, first note that since since $D_{v}=D_{v}^{\prime}+1$, we have (conditioned on $E$ )

$$
\frac{\sum_{v \in V_{n}} U_{v} D_{v}}{\sum_{v \in V_{n}} U_{v}}-\zeta^{*}=\frac{\sum_{v \in V_{n}} U_{v} D_{v}^{\prime}}{\sum_{v \in V_{n}} U_{v}}-\left(\zeta^{*}-1\right),
$$

so we may equivalently bound the first term in (143) with the left side of this equation replaced by the right side. For this, first note that since $D_{v}^{\prime} \sim \operatorname{Binomial}\left(\sum_{w \in V_{n}} N_{w}-n, \frac{1}{n}\right)$, we have

$$
\begin{aligned}
\mathbb{E}\left[\frac{\sum_{v \in V_{n}} U_{v} D_{v}^{\prime}}{\sum_{v \in V_{n}} U_{v}} 1(E)\right] & =\mathbb{E}\left[\frac{1}{\sum_{v \in V_{n}} U_{v}} \sum_{v \in V_{n}} U_{v} \mathbb{E}\left[D_{v}^{\prime} \mid\left\{N_{w}, U_{w}\right\}_{w \in V_{n}}\right] 1(E)\right] \\
& =\mathbb{E}\left[\frac{1}{\sum_{v \in V_{n}} U_{v}} \sum_{v \in V_{n}} U_{v} \frac{1}{n}\left(\sum_{w \in V} N_{w}-n\right) 1(E)\right]=\frac{1}{n} \mathbb{E}\left[\sum_{w \in V_{n}}\left(N_{w}-1\right) 1(E)\right],
\end{aligned}
$$

and so, diving by $\mathbb{P}[E]$, we obtain

$$
\mathbb{E}\left[\frac{\sum_{v \in V_{n}} U_{v} D_{v}^{\prime}}{\sum_{v \in V_{n}} U_{v}} \mid E\right]=\frac{1}{n} \mathbb{E}\left[\sum_{w \in V_{n}}\left(N_{w}-1\right) \mid E\right] .
$$

On the other hand, we have

$$
\begin{aligned}
\mathbb{E}\left[\sum_{w \in V_{n}}\left(N_{w}-1\right) \mid E\right]= & \frac{1}{\mathbb{P}[E]}\left(\mathbb{E}\left[\sum_{w \in V_{n}}\left(N_{w}-1\right)\right]-\mathbb{E}\left[\sum_{w \in V_{n}}\left(N_{w}-1\right) \mid E^{C}\right] \mathbb{P}\left[E^{C}\right]\right) \\
& =\frac{1}{1-n^{n(\kappa-1)}}\left(n\left(\mathbb{E}\left[N_{v}\right]-1\right)-n\left(\mathbb{E}\left[N_{v} \mid U_{v}=0\right]-1\right) n^{n(\kappa-1)}\right),
\end{aligned}
$$


so combining the previous two equations gives

$$
\mathbb{E}\left[\frac{\sum_{v \in V_{n}} U_{v} D_{v}^{\prime}}{\sum_{v \in V_{n}} U_{v}} \mid E\right]=\frac{1}{1-n^{n(\kappa-1)}}\left(\left(\mathbb{E}\left[N_{v}\right]-1\right)-\left(\mathbb{E}\left[N_{v} \mid U_{v}=0\right]-1\right) n^{n(\kappa-1)}\right) .
$$

Next, we write

$$
\begin{aligned}
\left|\mathbb{E}\left[\frac{\sum_{v \in V_{n}} U_{v} D_{v}^{\prime}}{\sum_{v \in V_{n}} U_{v}} \mid E\right]-\left(\zeta^{*}-1\right)\right| & \leq \frac{\left|\mathbb{E}\left[N_{v}\right]-\zeta^{*}\right|+n^{n(\kappa-1)}\left|\mathbb{E}\left[N_{v} \mid U_{v}=0\right]-\zeta^{*}\right|}{1-n^{n(\kappa-1)}} \\
& \leq \frac{\left|\mathbb{E}\left[N_{v}\right]-\eta_{1}\right|+n^{n(\kappa-1)}\left(\mathbb{E}\left[N_{v} \mid U_{v}=0\right]+\eta_{1}\right)}{1-n^{n(\kappa-1)}} \\
& \leq 2\left(\left|\mathbb{E}\left[N_{v}\right]-\eta_{1}\right|+n^{n(\kappa-1)}\left(\mathbb{E}\left[N_{v} \mid U_{v}=0\right]+\eta_{1}\right)\right)
\end{aligned}
$$

where the first inequality uses the triangle inequality and (144), the second uses the triangle inequality and $\zeta^{*}=\eta_{1}$ by definition, and the third holds for sufficiently large $n$ (since $n^{n(\kappa-1)} \rightarrow$ 0 as $n \rightarrow \infty)$. Now recall $\left|\mathbb{E}\left[N_{v}\right]-\eta_{1}\right|=O\left(n^{-\min \left\{l_{1}\left(c_{1}-2\right), l_{2}\left(c_{2}-1\right), 1-\kappa\right\}}\right)$ by (127). Furthermore, $\mathbb{E}\left[N_{v} \mid U_{v}=0\right]=O\left(n^{l_{2}\left(2-c_{2}\right)}\right)=O\left(n^{1-\kappa}\right)$ by Lemma 17 and the assumption $l_{2}\left(2-c_{2}\right)=1-\kappa$, and $\eta_{1}=O(1)$ by definition, so $n^{n(\kappa-1)}\left(\mathbb{E}\left[N_{v} \mid U_{v}=0\right]+\eta_{1}\right)=O\left(n^{(n-1)(\kappa-1)}\right)$, which is dominated by $O\left(n^{-\min \left\{l_{1}\left(c_{1}-2\right), l_{2}\left(c_{2}-1\right), 1-\kappa\right\}}\right)$ asymptotically. To summarize, we have argued

$$
\left|\mathbb{E}\left[\frac{\sum_{v \in V_{n}} U_{v} D_{v}^{\prime}}{\sum_{v \in V_{n}} U_{v}} \mid E\right]-\left(\zeta^{*}-1\right)\right|=O\left(n^{-\min \left\{l_{1}\left(c_{1}-2\right), l_{2}\left(c_{2}-1\right), 1-\kappa\right\}}\right) .
$$

Hence, by assumption $\gamma \in\left(0, \min \left\{l_{1}\left(c_{1}-2\right), l_{2}\left(c_{2}-1\right), 1-\kappa\right\}\right)$,

$$
\left|\mathbb{E}\left[\frac{\sum_{v \in V_{n}} U_{v} D_{v}^{\prime}}{\sum_{v \in V_{n}} U_{v}} \mid E\right]-\left(\zeta^{*}-1\right)\right| \leq n^{-\gamma} / 2
$$

holds for $n$ large. Thus, for such $n$, by Chebyshev's inequality,

$$
\begin{aligned}
P & {\left[\left|\frac{\sum_{v \in V_{n}} U_{v} D_{v}}{\sum_{v \in V_{n}} U_{v}}-\zeta^{*}\right|>n^{-\gamma} \mid E\right] \leq \mathbb{P}\left[\left|\frac{\sum_{v \in V_{n}} U_{v} D_{v}^{\prime}}{\sum_{v \in V_{n}} U_{v}}-\mathbb{E}\left[\frac{\sum_{v \in V_{n}} U_{v} D_{v}^{\prime}}{\sum_{v \in V_{n}} U_{v}} \mid E\right]\right|>n^{-\gamma} / 2 \mid E\right] } \\
& \leq 4 n^{2 \gamma}\left(\mathbb{E}\left[\left(\frac{\sum_{v \in V_{n}} U_{v} D_{v}^{\prime}}{\sum_{v \in V_{n}} U_{v}}\right)^{2} \mid E\right]-\left(\mathbb{E}\left[\frac{\sum_{v \in V_{n}} U_{v} D_{v}^{\prime}}{\sum_{v \in V_{n}} U_{v}} \mid E\right]\right)^{2}\right) .
\end{aligned}
$$

To further bound (145), let $E_{m}=\left\{\sum_{v \in V_{n}}\left(1-U_{v}\right)=m\right\}$ for each $m \in\{0,1, \ldots, n-1\}$; note $E=\cup_{m=0}^{n-1} E_{m}$. We can then write

$$
\begin{gathered}
\mathbb{E}\left[\left(\frac{\sum_{v \in V_{n}} U_{v} D_{v}^{\prime}}{\sum_{v \in V_{n}} U_{v}}\right)^{2} \mid E_{m}\right]=\frac{1}{(n-m)^{2}} \mathbb{E}\left[\left(\sum_{v \in V_{n}} U_{v} D_{v}^{\prime}\right)^{2} \mid E_{m}\right] \\
\leq \frac{1}{(n-m)^{2}} \mathbb{E}\left[\left(\sum_{v \in V_{n}} D_{v}^{\prime}\right)^{2} \mid E_{m}\right]=\frac{1}{(n-m)^{2}} \mathbb{E}\left[\left(\sum_{v \in V_{n}}\left(N_{v}-1\right)\right)^{2} \mid E_{m}\right] .
\end{gathered}
$$

We next claim

$$
\begin{aligned}
\mathbb{E}\left[\left(\sum_{v \in V_{n}}\left(N_{v}-1\right)\right)^{2} \mid E_{m}\right]=( & -m) \mathbb{E}\left[\left(N_{v}-1\right)^{2} \mid U_{v}=1\right]+m \mathbb{E}\left[\left(N_{v}-1\right)^{2} \mid U_{v}=0\right] \\
& +(n-m)(n-m-1)\left(\mathbb{E}\left[N_{v}-1 \mid U_{v}=1\right]\right)^{2} \\
& +m(m-1)\left(\mathbb{E}\left[N_{v}-1 \mid U_{v}=0\right]\right)^{2} \\
& +2(n-m) m \mathbb{E}\left[N_{v}-1 \mid U_{v}=1\right] \mathbb{E}\left[N_{v}-1 \mid U_{v}=0\right]
\end{aligned}
$$


which can seen by writing

$$
\begin{aligned}
\left(\sum_{v \in V_{n}}\left(N_{v}-1\right)\right)^{2}= & \sum_{v \in V_{n}: U_{v}=1}\left(N_{v}-1\right)^{2}+\sum_{v \in V_{n}: U_{v}=0}\left(N_{v}-1\right)^{2} \\
& +\sum_{v \neq w \in V_{n}: U_{v}=U_{w}=1}\left(N_{v}-1\right)\left(N_{w}-1\right) \\
& +\sum_{v \neq w \in V_{n}: U_{v}=U_{w}=0}\left(N_{v}-1\right)\left(N_{w}-1\right) \\
& +\sum_{v \neq w \in V_{n}: U_{v}=1, U_{w}=0}\left(N_{v}-1\right)\left(N_{w}-1\right)
\end{aligned}
$$

and recognizing that, conditioned on $E_{m}$, the summations in the previous equation will contain $n-m, m,(n-m)(n-m-1), m(m-1)$, and $2(n-m) m$ identically distributed terms, respectively, each with expected values corresponding to those written in (147). Combining (146) and (147), we obtain

$$
\begin{aligned}
& \mathbb{E}\left[\left(\frac{\sum_{v \in V_{n}} U_{v} D_{v}^{\prime}}{\sum_{v \in V_{n}} U_{v}}\right)^{2} \mid E_{m}\right] \leq \frac{\mathbb{E}\left[\left(N_{v}-1\right)^{2} \mid U_{v}=1\right]}{n-m}+\frac{m \mathbb{E}\left[\left(N_{v}-1\right)^{2} \mid U_{v}=0\right]}{(n-m)^{2}} \\
&+\left(\mathbb{E}\left[N_{v}-1 \mid U_{v}=1\right]\right)^{2}+\left(\frac{m \mathbb{E}\left[N_{v}-1 \mid U_{v}=0\right]}{n-m}\right)^{2} \\
&+\frac{2 m \mathbb{E}\left[N_{v}-1 \mid U_{v}=1\right] \mathbb{E}\left[N_{v}-1 \mid U_{v}=0\right]}{n-m} \\
&=\frac{\mathbb{E}\left[\left(N_{v}-1\right)^{2} \mid U_{v}=1\right]}{n-m}+\frac{m \mathbb{E}\left[\left(N_{v}-1\right)^{2} \mid U_{v}=0\right]}{(n-m)^{2}} \\
&+\left(\mathbb{E}\left[N_{v}-1 \mid U_{v}=1\right]+\frac{m \mathbb{E}\left[N_{v}-1 \mid U_{v}=0\right]}{n-m}\right)^{2} .
\end{aligned}
$$

Now assume $m \in\left(n^{\kappa}-n^{2 \kappa / 3}, n^{\kappa}+n^{2 \kappa / 3}\right)$. Then since $\mathbb{E}\left[\left(N_{v}-1\right)^{2} \mid U_{v}=1\right]=O(1), \mathbb{E}\left[\left(N_{v}-1\right)^{2} \mid U_{v}=\right.$ $0]=O\left(n^{l_{2}}\right)$ (which follows from (128)), we obtain

$$
\frac{\mathbb{E}\left[\left(N_{v}-1\right)^{2} \mid U_{v}=1\right]}{n-m}=O\left(n^{-1}\right), \quad \frac{m \mathbb{E}\left[\left(N_{v}-1\right)^{2} \mid U_{v}=0\right]}{(n-m)^{2}}=O\left(n^{\kappa+l_{2}-2}\right) .
$$

Furthermore, since $\mathbb{P}\left[U_{v}=1\right]=1-n^{\kappa-1}, \mathbb{P}\left[U_{v}=0\right]=n^{\kappa-1}$, we have for $m \in\left(n^{\kappa}-n^{2 \kappa / 3}, n^{\kappa}+n^{2 \kappa / 3}\right)$

$$
\begin{aligned}
& \frac{m \mathbb{E}\left[N_{v}-1 \mid U_{v}=0\right]}{n-m}=\frac{\mathbb{E}\left[N_{v}-1 \mid U_{v}=0\right] \mathbb{P}\left[U_{v}=0\right]}{\frac{n^{\kappa}}{m}-n^{\kappa-1}} \\
& <\frac{\mathbb{E}\left[N_{v}-1 \mid U_{v}=0\right] \mathbb{P}\left[U_{v}=0\right]}{\frac{n^{\kappa}}{n^{\kappa}+n^{2 \kappa / 3}}-n^{\kappa-1}}=\mathbb{E}\left[N_{v}-1 \mid U_{v}=0\right] \mathbb{P}\left[U_{v}=0\right]\left(\frac{1+n^{-\kappa / 3}}{1-n^{\kappa-1}-n^{(2 \kappa / 3)-1}}\right),
\end{aligned}
$$

and additionally,

$$
\begin{aligned}
\mathbb{E}\left[N_{v}-1 \mid U_{v}=1\right]= & \frac{\mathbb{E}\left[N_{v}-1 \mid U_{v}=1\right] \mathbb{P}\left[U_{v}=1\right]}{1-n^{\kappa-1}} \\
& <\mathbb{E}\left[N_{v}-1 \mid U_{v}=1\right] \mathbb{P}\left[U_{v}=1\right]\left(\frac{1+n^{-\kappa / 3}}{1-n^{\kappa-1}-n^{(2 \kappa / 3)-1}}\right) .
\end{aligned}
$$


Hence, combining (148), (149), (150), and (151), we have shown that for $m \in\left(n^{\kappa}-n^{2 \kappa / 3}, n^{\kappa}+n^{2 \kappa / 3}\right)$,

$$
\mathbb{E}\left[\left(\frac{\sum_{v \in V_{n}} U_{v} D_{v}^{\prime}}{\sum_{v \in V_{n}} U_{v}}\right)^{2} \mid E_{m}\right] \leq O\left(n^{-\min \left\{1,2-\kappa-l_{2}\right\}}\right)+\left(\mathbb{E}\left[N_{v}\right]-1\right)^{2}\left(\frac{1+n^{-\kappa / 3}}{1-n^{\kappa-1}-n^{(2 \kappa / 3)-1}}\right) .
$$

By the law of total expectation, we therefore obtain

$$
\begin{aligned}
\mathbb{E}\left[\left(\frac{\sum_{v \in V_{n}} U_{v} D_{v}^{\prime}}{\sum_{v \in V_{n}} U_{v}}\right)^{2} \mid E\right] \leq & O\left(n^{-\min \left\{1,2-\kappa-l_{2}\right\}}\right) \\
& +\left(\mathbb{E}\left[N_{v}\right]-1\right)^{2}\left(\frac{1+n^{-\kappa / 3}}{1-n^{\kappa-1}-n^{(2 \kappa / 3)-1}}\right) \\
& +n^{4} \mathbb{P}\left[\sum_{v \in V_{n}}\left(1-U_{v}\right) \notin\left(n^{\kappa}-n^{2 \kappa / 3}, n^{\kappa}+n^{2 \kappa / 3}\right) \mid E\right],
\end{aligned}
$$

where in the third line we have used the coarse bound

$$
\sum_{v \in V_{n}} \frac{U_{v}}{\sum_{w \in V_{n}} U_{w}} D_{v}^{\prime} \leq \sum_{v \in V_{n}} D_{v}^{\prime} \leq n^{2}
$$

with the fraction well-defined conditioned on $E$. Furthermore,

$$
\mathbb{P}\left[\sum_{v \in V_{n}}\left(1-U_{v}\right) \notin\left(n^{\kappa}-n^{2 \kappa / 3}, n^{\kappa}+n^{2 \kappa / 3}\right) \mid E\right] \leq \frac{1}{\mathbb{P}[E]} \mathbb{P}\left[\sum_{v \in V_{n}}\left(1-U_{v}\right) \notin\left(n^{\kappa}-n^{2 \kappa / 3}, n^{\kappa}+n^{2 \kappa / 3}\right)\right],
$$

which, using the fact that $\mathbb{P}[E] \rightarrow 1$ as $n \rightarrow \infty$, and Lemma 18 , shows that the probability term in (152) decays exponentially fast in $n$. Hence, multiplying this probability term by $n^{4}$ yields a term dominated by $O\left(n^{-\min \left\{1,2-\kappa-l_{2}\right\}}\right)$. In other words, we have shown

$$
\mathbb{E}\left[\left(\frac{\sum_{v \in V_{n}} U_{v} D_{v}^{\prime}}{\sum_{v \in V_{n}} U_{v}}\right)^{2} \mid E\right] \leq O\left(n^{-\min \left\{1,2-\kappa-l_{2}\right\}}\right)+\left(\mathbb{E}\left[N_{v}\right]-1\right)^{2}\left(\frac{1+n^{-\kappa / 3}}{1-n^{\kappa-1}-n^{(2 \kappa / 3)-1}}\right) .
$$

Now combining the previous equation with (144), we obtain

$$
\begin{aligned}
\mathbb{E} & {\left[\left(\frac{\sum_{v \in V_{n}} U_{v} D_{v}^{\prime}}{\sum_{v \in V_{n}} U_{v}}\right)^{2} \mid E\right]-\left(\mathbb{E}\left[\frac{\sum_{v \in V_{n}} U_{v} D_{v}^{\prime}}{\sum_{v \in V_{n}} U_{v}} \mid E\right]\right)^{2} } \\
& \leq\left(\mathbb{E}\left[N_{v}\right]-1\right)^{2}\left(\frac{1+n^{-\kappa / 3}}{1-n^{\kappa-1}-n^{(2 \kappa / 3)-1}}-\frac{1}{\left(1-n^{n(\kappa-1)}\right)^{2}}\right)+O\left(n^{-\min \left\{1,2-\kappa-l_{2}\right\}}\right),
\end{aligned}
$$

where we have dropped the terms from (144) with factors of $n^{n(\kappa-1)}$, since all such terms decay faster than $O\left(n^{-\min \left\{1,2-\kappa-l_{2}\right\}}\right)$. Furthermore, we note

$$
\frac{1+n^{-\kappa / 3}}{1-n^{\kappa-1}-n^{(2 \kappa / 3)-1}}-\frac{1}{\left(1-n^{n(\kappa-1)}\right)^{2}} \leq \frac{n^{-\kappa / 3}+n^{\kappa-1}+n^{(2 \kappa / 3)-1}}{\left(1-n^{\kappa-1}-n^{(2 \kappa / 3)-1}\right)\left(1-n^{n(\kappa-1)}\right)^{2}}=O\left(n^{-\min \{\kappa / 3,1-\kappa\}}\right),
$$

and so, combining (155) and (156) gives

$$
\begin{aligned}
\mathbb{E}\left[\left(\frac{\sum_{v \in V_{n}} U_{v} D_{v}^{\prime}}{\sum_{v \in V_{n}} U_{v}}\right)^{2} \mid E\right]-\left(\mathbb{E}\left[\frac{\sum_{v \in V_{n}} U_{v} D_{v}^{\prime}}{\sum_{v \in V_{n}} U_{v}} \mid E\right]\right)^{2} & =O\left(n^{-\min \left\{1,2-\kappa-l_{2}, \kappa / 3,1-\kappa\right\}}\right) \\
& =O\left(n^{-\min \{\kappa / 3,1-\kappa\}}\right),
\end{aligned}
$$


where the first equality also uses $\left(\mathbb{E}\left[N_{v}\right]-1\right)^{2}=O(1)$ and the second also uses $l_{2}, \kappa \in(0,1)$ to simplify the min expression. Finally, combining (143), (145), and (157) completes the proof.

Lemma 23. Let $\lambda^{*}=z\left(c_{1}-1\right) / z\left(c_{1}\right)$, which is well-defined by assumption $c_{1}>3$. Then if $\gamma \in$ $\left(0, l_{1}\left(c_{1}-2\right)\right)$, we have

$$
\mathbb{P}\left[\left|\frac{\sum_{v \in V_{n}} U_{v} N_{v}}{\sum_{v \in V_{n}} U_{v}}-\lambda^{*}\right|>n^{-\gamma}\right]=O\left(n^{2 \gamma-1}\right) .
$$

Proof. We first define $E$ as in the proof of Lemma 22 and write

$$
\mathbb{P}\left[\left|\frac{\sum_{v \in V_{n}} U_{v} N_{v}}{\sum_{v \in V_{n}} U_{v}}-\lambda^{*}\right|>n^{-\gamma}\right] \leq \mathbb{P}\left[\left|\frac{\sum_{v \in V_{n}} U_{v} N_{v}}{\sum_{v \in V_{n}} U_{v}}-\lambda^{*}\right|>n^{-\gamma} \mid E\right]+n^{n(\kappa-1)} .
$$

It remains to bound the first term in (158). For this, we first note

$$
\mathbb{E}\left[U_{v} N_{v} \mid\left\{U_{w}\right\}_{w \in V_{n}}\right]=U_{v} \frac{\sum_{i=1}^{\left\lceil n^{l_{1}}\right\rceil} i^{1-c_{1}}}{\sum_{i=1}^{\left\lceil n^{l_{1}}\right\rceil} i^{-c_{1}}},
$$

which holds because both sides are zero when $U_{v}=0$, and since $\mathbb{P}\left[N_{v}=i\right]=i^{-c_{1}} / \sum_{j=1}^{\left\lceil n^{l_{1}}\right\rceil} j^{-c_{1}}$ when $U_{v}=1$ (with $U_{v}, N_{v}$ both independent of $U_{w}, w \neq v$ ). Therefore,

$$
\begin{aligned}
\mathbb{E}\left[\frac{\sum_{v \in V_{n}} N_{v} U_{v}}{\sum_{v \in V_{n}} U_{v}} 1(E)\right] & =\mathbb{E}\left[\frac{1}{\sum_{v \in V_{n}} U_{v}} \sum_{v \in V_{n}} \mathbb{E}\left[U_{v} N_{v} \mid\left\{U_{w}\right\}_{w \in V_{n}}\right] 1(E)\right] \\
& =\mathbb{E}\left[\frac{1}{\sum_{v \in V_{n}} U_{v}} \sum_{v \in V_{n}}\left(U_{v} \frac{\sum_{i=1}^{\left\lceil n^{l_{1}}\right\rceil} i^{1-c_{1}}}{\sum_{i=1}^{\left\lceil n^{l_{1}}\right\rceil} i^{-c_{1}}}\right) 1(E)\right]=\frac{\left.\sum_{i=1}^{\left\lceil n_{1}\right.}\right\rceil i^{1-c_{1}}}{\sum_{i=1}^{\left\lceil n^{l_{1}}\right\rceil} i^{-c_{1}}} \mathbb{P}[E],
\end{aligned}
$$

and so, dividing by $\mathbb{P}[E]$ on both sides,

$$
\mathbb{E}\left[\frac{\sum_{v \in V_{n}} N_{v} U_{v}}{\sum_{v \in V_{n}} U_{v}} \mid E\right]=\frac{\sum_{i=1}^{\left\lceil n^{l_{1}}\right\rceil} i^{1-c_{1}}}{\sum_{i=1}^{\left\lceil n^{l_{1}}\right\rceil} i^{-c_{1}}}=\mathbb{E}\left[N_{v} \mid U_{v}=1\right] .
$$

Furthermore, by an argument similar to that leading to (119),

$$
\begin{aligned}
& 0 \leq \frac{z\left(c_{1}-1\right)}{z\left(c_{1}\right)}-\frac{\sum_{i=1}^{\left\lceil n^{l_{1}}\right\rceil} i^{1-c_{1}}}{\left.\sum_{i=1}^{\left\lceil n_{1}\right.}\right\rceil i^{-c_{1}}} \leq \frac{z\left(c_{1}-1\right)-\sum_{i=1}^{\left\lceil n^{l_{1}}\right\rceil} i^{1-c_{1}}}{z\left(c_{1}\right)} \leq \frac{\left\lceil n^{l_{1}}\right\rceil^{2-c_{1}}}{z\left(c_{1}\right)} \\
& \Rightarrow\left|\frac{z\left(c_{1}-1\right)}{z\left(c_{1}\right)}-\frac{\sum_{i=1}^{\left\lceil n^{l_{1}}\right\rceil} i^{1-c_{1}}}{\sum_{i=1}^{\left\lceil n_{1} l_{1}\right.} i^{-c_{1}}}\right|=O\left(n^{l_{1}\left(2-c_{1}\right)}\right) \text {. }
\end{aligned}
$$

Hence, by assumption $\gamma \in\left(0, l_{1}\left(c_{1}-2\right)\right),(160)$ is bounded by $n^{-\gamma} / 2$ for $n$ sufficiently large; for such $n$, we can then use Chebyshev's inequality to obtain

$$
\begin{aligned}
& \mathbb{P}\left[\left|\frac{\sum_{v \in V_{n}} N_{v} U_{v}}{\sum_{v \in V_{n}} U_{v}}-\lambda^{*}\right|>n^{-\gamma} \mid E\right] \\
& \quad \leq \mathbb{P}\left[\left|\frac{\sum_{v \in V_{n}} N_{v} U_{v}}{\sum_{v \in V_{n}} U_{v}}-\mathbb{E}\left[\frac{\sum_{v \in V_{n}} N_{v} U_{v}}{\sum_{v \in V_{n}} U_{v}} \mid E\right]\right|>n^{-\gamma} / 2 \mid E\right] \leq 4 n^{2 \gamma} \operatorname{Var}\left(\frac{\sum_{v \in V_{n}} N_{v} U_{v}}{\sum_{v \in V_{n}} U_{v}} \mid E\right) .
\end{aligned}
$$


It remains to bound the variance term. For this, define the event $E_{m}, m \in\{1,2, \ldots, n-1\}$ as in the proof of Lemma 22. Then

$$
\begin{aligned}
\mathbb{E}\left[\left(\frac{\sum_{v \in V_{n}} N_{v} U_{v}}{\sum_{v \in V_{n}} U_{v}}\right)^{2} \mid E_{m}\right]= & \frac{1}{(n-m)^{2}} \mathbb{E}\left[\sum_{v \in V_{n}: U_{v}=1} N_{v}^{2} \mid E_{m}\right] \\
& \quad+\frac{1}{(n-m)^{2}} \mathbb{E}\left[\sum_{v, w \in V_{n}: v \neq w, U_{v}=U_{w}=1} N_{v} N_{w} \mid E_{m}\right] \\
= & \frac{1}{n-m} \mathbb{E}\left[N_{v}^{2} \mid U_{v}=1\right]+\frac{(n-m-1)}{n-m}\left(\mathbb{E}\left[N_{v} \mid U_{v}=1\right]\right)^{2} \\
& <\frac{1}{n-m} \mathbb{E}\left[N_{v}^{2} \mid U_{v}=1\right]+\left(\mathbb{E}\left[N_{v} \mid U_{v}=1\right]\right)^{2},
\end{aligned}
$$

which follows from an argument similar to (147) in the proof of Lemma 22. Hence, for $m \in$ $\left(n^{\kappa}-n^{2 \kappa / 3}, n^{\kappa}+n^{2 \kappa / 3}\right)$, we obtain

$$
\begin{aligned}
\mathbb{E}\left[\left(\frac{\sum_{v \in V_{n}} N_{v} U_{v}}{\sum_{v \in V_{n}} U_{v}}\right)^{2} \mid E_{m}\right] & <\frac{1}{n-n^{\kappa}-n^{2 \kappa / 3}} \mathbb{E}\left[N_{v}^{2} \mid U_{v}=1\right]+\left(\mathbb{E}\left[N_{v} \mid U_{v}=1\right]\right)^{2} \\
& =O\left(n^{-1}\right)+\left(\mathbb{E}\left[N_{v} \mid U_{v}=1\right]\right)^{2},
\end{aligned}
$$

where we have used the fact that $\mathbb{E}\left[N_{v}^{2} \mid U_{v}=1\right]=O(1)$ (see (128)). Therefore, by the law of total probability and an argument analogous to (153) in the proof of Lemma 22,

$$
\begin{aligned}
\mathbb{E}\left[\left(\frac{\sum_{v \in V_{n}} N_{v} U_{v}}{\sum_{v \in V_{n}} U_{v}}\right)^{2} \mid E\right]< & O\left(n^{-1}\right)+\left(\mathbb{E}\left[N_{v} \mid U_{v}=1\right]\right)^{2} \\
& +n^{4} \mathbb{P}\left[\sum_{v \in V_{n}}\left(1-U_{v}\right) \notin\left(n^{\kappa}-n^{2 \kappa / 3}, n^{\kappa}+n^{2 \kappa / 3}\right) \mid E\right] \\
= & O\left(n^{-1}\right)+\left(\mathbb{E}\left[N_{v} \mid U_{v}=1\right]\right)^{2},
\end{aligned}
$$

where we have also used the fact that the probability term decays exponentially fast in $n$ (see (154) and the subsequent text in the proof of Lemma 22). Substituting into (161) gives

$$
\begin{aligned}
& \mathbb{P}\left[\left|\frac{\sum_{v \in V_{n}} N_{v} U_{v}}{\sum_{v \in V_{n}} U_{v}}-\lambda^{*}\right|>n^{-\gamma} \mid E\right] \\
& \quad \leq 4 n^{2 \gamma}\left(O\left(n^{-1}\right)+\left(\mathbb{E}\left[N_{v} \mid U_{v}=1\right]\right)^{2}-\left(\mathbb{E}\left[\frac{\sum_{v \in V_{n}} N_{v} U_{v}}{\sum_{v \in V_{n}} U_{v}} \mid E\right]\right)^{2}\right)=O\left(n^{2 \gamma-1}\right),
\end{aligned}
$$

where the second line uses (159). Finally, combining the previous equation with (158) completes the proof.

LEMMA 24. Let

$$
p=\frac{\frac{z\left(c_{1}-1\right)}{z\left(c_{1}\right)}}{\frac{z\left(c_{1}-1\right)}{z\left(c_{1}\right)}+\frac{1}{\left(2-c_{2}\right) z\left(c_{2}\right)}},
$$

which is well-defined since $c_{1}>3, c_{2}>1$ by assumption. Then if $\gamma \in\left(0, \min \left\{l_{1}\left(c_{1}-2\right), l_{2}\left(c_{2}-1\right), 1-\right.\right.$ $\kappa, \kappa / 3\})$, we have

$$
\mathbb{P}\left[\left|\frac{\sum_{v \in V_{n}} U_{v} N_{v}}{\sum_{v \in V_{n}} N_{v}}-p\right|>n^{-\gamma}\right]=O\left(n^{l_{2}+2 \gamma-1}\right) .
$$


Proof. We first define

$$
\begin{gathered}
\mu_{1, n}=\mathbb{E}\left[U_{v} N_{v}\right]=\left(1-n^{\kappa-1}\right) \frac{\sum_{i=1}^{\left\lceil n^{l_{1}}\right\rceil} i^{1-c_{1}}}{\sum_{i=1}^{\left\lceil n_{1}\right\rceil} i^{-c_{1}}}, \quad \mu_{1}=\frac{z\left(c_{1}-1\right)}{z\left(c_{1}\right)} \\
\mu_{2, n}=\mathbb{E}\left[\left(1-U_{v}\right) N_{v}\right]=n^{\kappa-1} \frac{\sum_{i=1}^{\left\lceil n_{2}\right\rceil} i^{1-c_{2}}}{\sum_{i=1}^{\left\lceil n_{2}\right\rceil} i^{-c_{2}}}, \quad \mu_{2}=\frac{1}{\left(2-c_{2}\right) z\left(c_{2}\right)},
\end{gathered}
$$

so that $p=\frac{\mu_{1}}{\mu_{1}+\mu_{2}}$. Additionally, recall by (119) and (126) we have

$$
\left|\mu_{1, n}-\mu_{1}\right|=O\left(n^{-\min \left\{l_{1}\left(c_{1}-2\right), 1-\kappa\right\}}\right), \quad\left|\mu_{2, n}-\mu_{2}\right|=O\left(n^{-\min \left\{l_{2}\left(c_{2}-1\right), 1-\kappa\right\}}\right) .
$$

Next, define $E_{m}=\left\{\sum_{v \in V_{n}}\left(1-U_{v}\right)=m\right\}$ and note that, conditioned on $E_{m}, \sum_{v \in V_{n}} U_{v} N_{v}$ has the same distribution as $\sum_{i=1}^{n-m} X_{i}$, where $\left\{X_{i}\right\}_{i=1}^{n-m}$ is an i.i.d. set of random variables, each satisfying

$$
\mathbb{P}\left[X_{i}=x\right]=\frac{x^{-c_{1}}}{\sum_{j=1}^{\left\lceil n^{l_{1}}\right\rceil} j^{-c_{1}}} .
$$

Similarly, conditioned on $E_{m}, \sum_{v \in V_{n}}\left(1-U_{v}\right) N_{v}$ has the same distribution as $\sum_{i=1}^{m} Y_{i}$, where $\left\{Y_{i}\right\}_{i=1}^{m}$ is an i.i.d. set of random variables, each with distribution

$$
\mathbb{P}\left[Y_{i}=y\right]=\frac{y^{-c_{2}}}{\sum_{j=1}^{\left[n^{l_{2}}\right\rceil} j^{-c_{2}}} .
$$

It follows that

$$
\begin{aligned}
& \mathbb{P}\left[\frac{\sum_{v \in V_{n}} U_{v} N_{v}}{\sum_{v \in V_{n}} N_{v}}>p+n^{-\gamma} \mid E_{m}\right]=\mathbb{P}\left[\frac{\sum_{i=1}^{n-m} X_{i}}{\sum_{i=1}^{n-m} X_{i}+\sum_{i=1}^{m} Y_{i}}>p+n^{-\gamma}\right] \\
& \quad \leq \mathbb{P}\left[\frac{\sum_{i=1}^{n-m} X_{i}}{\sum_{i=1}^{n-m} X_{i}+\sum_{i=1}^{m} Y_{i}}>p+n^{-\gamma} \mid \frac{\sum_{i=1}^{n-m} X_{i}}{n\left(\mu_{1}+\mu_{2}\right)} \leq p+n^{-\gamma}\right]+\mathbb{P}\left[\frac{\sum_{i=1}^{n-m} X_{i}}{n\left(\mu_{1}+\mu_{2}\right)}>p+n^{-\gamma}\right] \\
& \quad \leq \mathbb{P}\left[\frac{\sum_{i=1}^{m} Y_{i}}{n\left(\mu_{1}+\mu_{2}\right)}<1-p-n^{-\gamma}\right]+\mathbb{P}\left[\frac{\sum_{i=1}^{n-m} X_{i}}{n\left(\mu_{1}+\mu_{2}\right)}>p+n^{-\gamma}\right],
\end{aligned}
$$

where the first equality uses the observation of the previous paragraph and the first inequality simply bounds two probability terms by 1 ; the second inequality holds since $\frac{\sum_{i=1}^{n-m} X_{i}}{\sum_{i=1}^{n-m} X_{i}+\sum_{i=1}^{m} Y_{i}}$ is increasing in $\sum_{i=1}^{n-m} X_{i}$, since each $Y_{i}$ is independent of $\sum_{i=1}^{n-m} X_{i}$, and by rearranging terms. By a similar argument, we obtain the lower tail

$$
\mathbb{P}\left[\frac{\sum_{v \in V_{n}} U_{v} N_{v}}{\sum_{v \in V_{n}} N_{v}}<p-n^{-\gamma} \mid E_{m}\right] \leq \mathbb{P}\left[\frac{\sum_{i=1}^{m} Y_{i}}{n\left(\mu_{1}+\mu_{2}\right)}>1-p+n^{-\gamma}\right]+\mathbb{P}\left[\frac{\sum_{i=1}^{n-m} X_{i}}{n\left(\mu_{1}+\mu_{2}\right)}<p-n^{-\gamma}\right] .
$$

Combining (163) and (164), we can then obtain

$$
\begin{aligned}
\mathbb{P} & {\left[\left|\frac{\sum_{v \in V_{n}} U_{v} N_{v}}{\sum_{v \in V_{n}} N_{v}}-p\right|>n^{-\gamma} \mid E_{m}\right] } \\
& \leq \mathbb{P}\left[\left|\frac{1}{n} \sum_{i=1}^{n-m} X_{i}-\mu_{1}\right|>\left(\mu_{1}+\mu_{2}\right) n^{-\gamma}\right]+\mathbb{P}\left[\left|\frac{1}{n} \sum_{i=1}^{m} Y_{i}-\mu_{2}\right|>\left(\mu_{1}+\mu_{2}\right) n^{-\gamma}\right],
\end{aligned}
$$

where we also used $p=\frac{\mu_{1}}{\mu_{1}+\mu_{2}}$ (which holds by definition). 
Next, we assume $m \in\left(n^{\kappa}-n^{2 \kappa / 3}, n^{\kappa}+n^{2 \kappa / 3}\right)$. Then, using (162),

$$
\begin{aligned}
\mid \mathbb{E} & {\left[\frac{1}{n} \sum_{i=1}^{n-m} X_{i}\right]-\mu_{1}|=| \frac{1}{n}(n-m) \frac{\mu_{1, n}}{1-n^{\kappa-1}}-\mu_{1}|=| \mu_{1, n}-\mu_{1}+\frac{n^{\kappa-1}-\frac{m}{n}}{1-n^{\kappa-1}} \mu_{1, n} \mid } \\
& \leq\left|\mu_{1, n}-\mu_{1}\right|+\left|\frac{n^{\kappa-1}-\frac{m}{n}}{1-n^{\kappa-1}}\right|\left|\mu_{1, n}\right| \leq\left|\mu_{1, n}-\mu_{1}\right|+\frac{n^{\kappa-1}}{1-n^{\kappa-1}} \mu_{1}=O\left(n^{-\min \left\{l_{1}\left(c_{1}-2\right), 1-\kappa\right\}}\right), \\
\mid \mathbb{E} & {\left[\frac{1}{n} \sum_{i=1}^{m} Y_{i}\right]-\mu_{2}|=| \frac{1}{n} m \frac{\mu_{2, n}}{n^{\kappa-1}}-\mu_{2}|=| \mu_{2, n}-\mu_{2}+\frac{m-n^{\kappa}}{n^{\kappa}} \mu_{2, n} \mid } \\
& \leq\left|\mu_{2, n}-\mu_{2}\right|+\left|\frac{m-n^{\kappa}}{n^{\kappa}}\right|\left|\mu_{2, n}\right| \leq\left|\mu_{2, n}-\mu_{2}\right|+n^{-\kappa / 3} \mu_{2}=O\left(n^{-\min \left\{l_{2}\left(c_{2}-1\right), 1-\kappa, \kappa / 3\right\}}\right) .
\end{aligned}
$$

Now by assumption $\gamma \in\left(0, \min \left\{l_{1}\left(c_{1}-2\right), l_{2}\left(c_{2}-1\right), 1-\kappa, \kappa / 3\right\}\right),(165)$ and $(166)$ are each bounded by $\left(\mu_{1}+\mu_{2}\right) n^{-\gamma} / 2$ for large enough $n$; hence, using Chebyshev's inequality, we can obtain

$$
\begin{aligned}
\mathbb{P}\left[\left|\frac{1}{n} \sum_{i=1}^{n-m} X_{i}-\mu_{1}\right|>\left(\mu_{1}+\mu_{2}\right) n^{-\gamma}\right] & \leq \mathbb{P}\left[\left|\frac{1}{n} \sum_{i=1}^{n-m} X_{i}-\frac{n-m}{n} \mathbb{E}\left[X_{i}\right]\right|>\left(\mu_{1}+\mu_{2}\right) n^{-\gamma} / 2\right] \\
& \leq \frac{4 n^{2 \gamma}}{\left(\mu_{1}+\mu_{2}\right)^{2}} \frac{n-m}{n^{2}} \mathbb{E}\left[X_{i}^{2}\right]=O\left(n^{2 \gamma-1}\right),
\end{aligned}
$$

where the equality holds for $m \in\left(n^{\kappa}-n^{2 \kappa / 3}, n^{\kappa}+n^{2 \kappa / 3}\right)$ and uses the fact that $\mathbb{E}\left[X_{i}^{2}\right]=O(1)$ (see (128)). Similarly, we have

$$
\begin{aligned}
\mathbb{P}\left[\left|\frac{1}{n} \sum_{i=1}^{m} Y_{i}-\mu_{2}\right|>\left(\mu_{1}+\mu_{2}\right) n^{-\gamma}\right] & \leq \mathbb{P}\left[\left|\frac{1}{n} \sum_{i=1}^{m} Y_{i}-\frac{m}{n} \mathbb{E}\left[Y_{i}\right]\right|>\left(\mu_{1}+\mu_{2}\right) n^{-\gamma} / 2\right] \\
& \leq \frac{4 n^{2 \gamma}}{\left(\mu_{1}+\mu_{2}\right)^{2}} \frac{m}{n^{2}} \mathbb{E}\left[Y_{i}^{2}\right]=O\left(n^{l_{2}+2 \gamma-1}\right),
\end{aligned}
$$

where the equality holds for $m \in\left(n^{\kappa}-n^{2 \kappa / 3}, n^{\kappa}+n^{2 \kappa / 3}\right)$, uses $\mathbb{E}\left[Y_{i}^{2}\right]=O\left(n^{l_{2}\left(3-c_{2}\right)}\right)$ (see (128)), and uses the assumption $l_{2}\left(2-c_{2}\right)=1-\kappa$. To summarize, we have for $m \in\left(n^{\kappa}-n^{2 \kappa / 3}, n^{\kappa}+n^{2 \kappa / 3}\right)$,

$$
\mathbb{P}\left[\left|\frac{\sum_{v \in V_{n}} U_{v} N_{v}}{\sum_{v \in V_{n}} N_{v}}-p\right|>n^{-\gamma} \mid E_{m}\right]=O\left(n^{l_{2}+2 \gamma-1}\right) .
$$

Hence, by the law of total probability,

$$
\begin{aligned}
\mathbb{P}\left[\left|\frac{\sum_{v \in V_{n}} U_{v} N_{v}}{\sum_{v \in V_{n}} N_{v}}-p\right|>n^{-\gamma}\right] & \leq O\left(n^{l_{2}+2 \gamma-1}\right)+\mathbb{P}\left[\sum_{v \in V_{n}}\left(1-U_{v}\right) \notin\left(n^{\kappa}-n^{2 \kappa / 3}, n^{\kappa}+n^{2 \kappa / 3}\right)\right] \\
& =O\left(n^{l_{2}+2 \gamma-1}\right),
\end{aligned}
$$

where the second line uses Lemma 18.

\section{I.3 Proof of Proposition 2}

Let us define

$$
\begin{aligned}
& \bar{\gamma}_{1}=\min \left\{l_{1}\left(c_{1}-3\right), l_{2}\left(c_{2}-1\right), 1-\kappa, \frac{\kappa}{3}\right\}>0, \\
& \bar{\gamma}_{2}=\min \left\{\frac{1-l_{2}}{2}, \frac{1-l_{1}\left(5-c_{1}\right)}{2}, \frac{1-\kappa}{2}, \frac{\kappa}{6}\right\}>0,
\end{aligned}
$$


where the inequalities hold by the assumptions $l_{1} \in\left(0, \frac{1}{5-c_{1}}\right), c_{1} \in(3,4), l_{2} \in(0,1), c_{2} \in(1,2)$, and $\kappa \in(0,1)$. Now if we choose $\gamma \in\left(0, \bar{\gamma}_{1}\right)$, the assumptions of Lemmas $19-24$ hold; the tail bounds in Lemmas 19-24 then imply

$$
\mathbb{P}\left[\Omega_{n}^{C}\right] \leq \sum_{i=1}^{6} \mathbb{P}\left[\Omega_{n, i}^{C}\right]=O\left(n^{2\left(\gamma-\bar{\gamma}_{2}\right)}\right) .
$$

If we also choose $\gamma \in\left(0, \bar{\gamma}_{2}\right)$, then there exists $\delta>0$ such that $\mathbb{P}\left[\Omega_{n}^{C}\right]=O\left(n^{-\delta}\right)$, as required by Assumption 1. Additionally, the constants defined in Lemmas 19-24 satisfy $\eta_{1}, \eta_{2}, \eta_{3}, \zeta^{*}, \lambda^{*} \in(0, \infty)$, $p \in(0,1)$, as required by Assumption 1 . Finally, $\mathbb{E}\left[\sum_{v \in V_{n}}\left(1-U_{v}\right)\right]=O\left(n^{\kappa}\right)$ for $\kappa \in(0,1)$, as required by Assumption 2 .

Received August 2018; revised February 2019; accepted April 2019 